\title{
EXPLORING MECHANICAL ASSONANCE FOR \\ IMPACT ENERGY HARVESTING USING ACOUSTIC \\ METAMATERIALS
}

\author{
By \\ MILKYAS AFEWORK \\ Bachelor of Science in Mechanical Engineering \\ Oklahoma State University \\ Stillwater, Oklahoma \\ 2018
}
Submitted to the Faculty of the
Graduate College of the Oklahoma State University in partial fulfillment of the requirements for the Degree of MASTER OF SCIENCE

December, 2020 


\section{EXPLORING MECHANICAL ASSONANCE FOR IMPACT ENERGY HARVESTING USING ACOUSTIC \\ METAMATERIALS}

Thesis Approved:

Dr. James M. Manimala

Thesis Adviser

Dr. He Bai

Dr. Shuodao Wang 
Name: MILKYAS AFEWORK

Date of Degree: DECEMBER, 2020

\title{
Title of Study: EXPLORING MECHANICAL ASSONANCE FOR IMPACT ENERGY HARVESTING USING ACOUSTIC METAMATERIALS
}

\section{Major Field: MECHANICAL AND AEROSPACE ENGINEERING}

\begin{abstract}
Acoustic metamaterials are engineered to possess unique dynamic properties that are not commonly found in nature. It has been demonstrated that customizing the characteristics of their local features can help optimize their dynamic performance under specific loading conditions. Drawing inspiration from the literary device called 'assonance,' the term 'mechanical assonance' may be ascribed to the dynamic phenomenon realized by sequencing oscillators with tuned responses within a waveguide to engineer a prescribed wave transformation across it. In this context, assonance provides a framework to utilize resonant local features within a host structure or material and interactive mechanisms thereof as building blocks to create enriched functionalities for acoustic metamaterials. Using a discrete element representation for an acoustic metamaterial barrier (AMB), a numerical case study is conducted to ascertain parametric dependence for assonant mechanisms related to resonator frequencies, their sequencing, and host material stiffness. Normalized metrics are extracted to estimate transmitted pulse mitigation under impact-type loading. It is found that resonator sets with octave spacing having the number of resonators of a specific frequency proportional to that frequency's amplitude in the input spectrum is desirable for lower transmissibility. Further, sequencing the lowest frequency resonator set closest to the incident-side gives better performance. Engineering a high degree of impedance mismatch between host material sections is also preferable. The energy sequestered by the local resonators is amenable to harvesting by utilizing the resonator's mass as the multifunctional kernel for a linear electromagnetic generator. A multiphysical model is developed to predict the harvested electric voltage and power from the AMB and validated using proof-of-concept experiments. Finally, various coil placement and voltage rectification schemes are also studied using simulations to ascertain preferable design configurations.
\end{abstract}




\section{TABLE OF CONTENTS}

Chapter $\quad$ Page

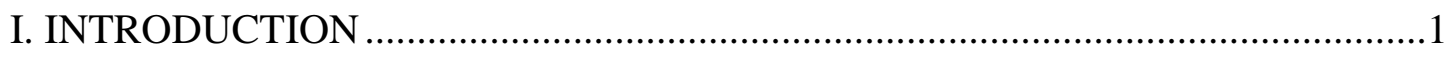

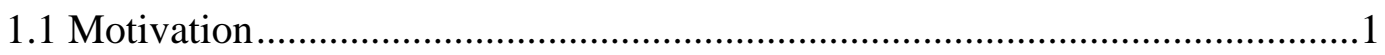

1.2 Literature Review..................................................................................

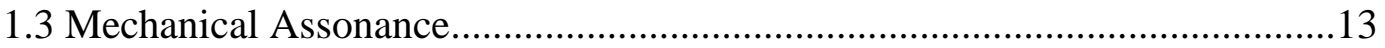

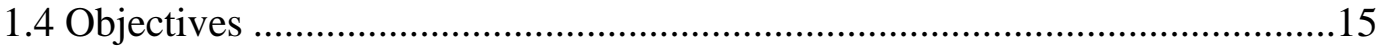

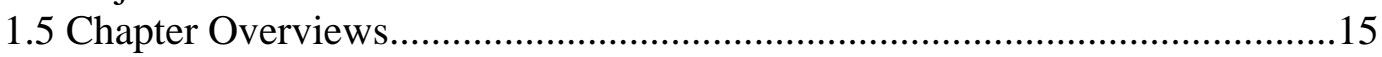

II. THEORETICAL MODELING FOR LOCALLY RESONANT ACOUSTIC

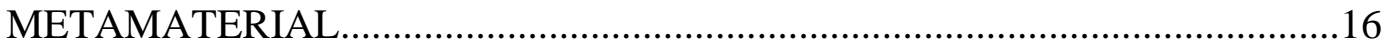

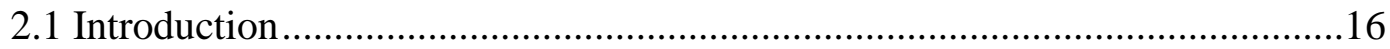

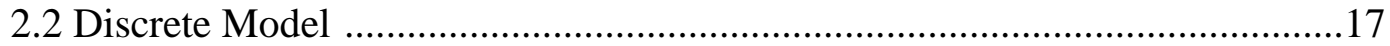

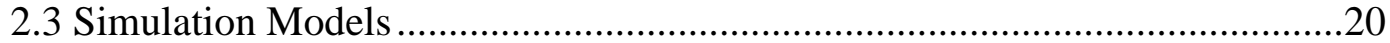

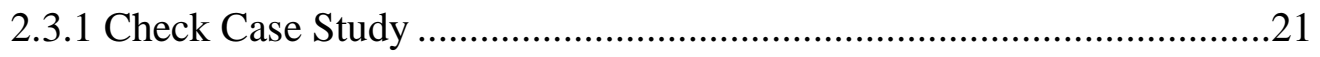

2.3.2 Baseline Case Study ........................................................................21

2.3.3 Two-Parameter Study .....................................................................23

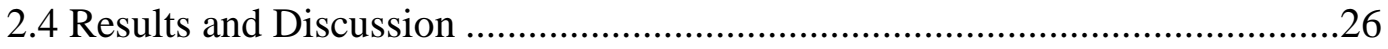

2.4.1 Check Case Study ............................................................................26

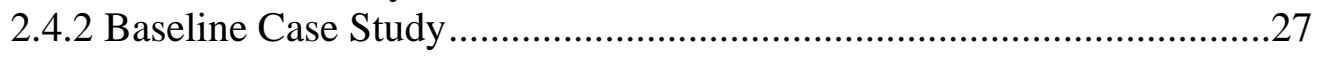

2.4.3 Two Parameter Study ..................................................................28

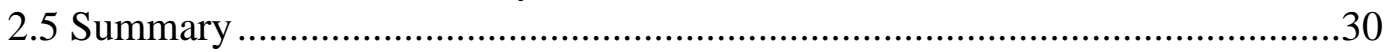

III. ASSONANCE-BASED DESIGN CASE STUDY ...............................................

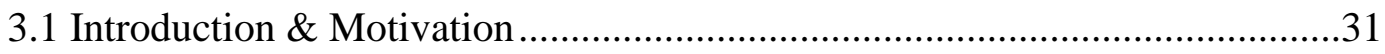

3.2 Numerical Parametric Studies.................................................................

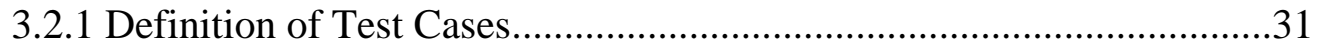

3.2.2.1 Resonator-Type Variation Case ...................................................32

3.2.2.1.1 Single Resonator-Type Case ..........................................32

3.2.2.1.2 Two Resonator-Type Case..............................................33

3.2.2.1.3 Four Resonator-Type Case …………….......................33

3.2.2.1.4 N Distinct Resonator-Type Case ....................................34

3.2.2.2 Resonator Sequence Variation Case ...............................................35

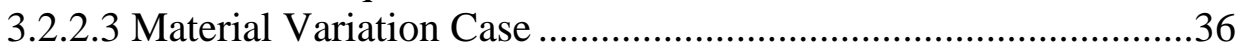

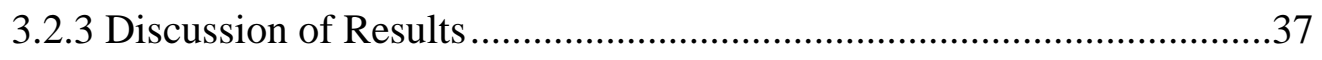




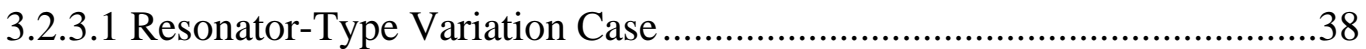

3.2.3.1.1 Single Resonator-Type Case.....................................38

3.2.3.1.2 Two Resonator-Type Case......................................40

3.2.3.1.3 Four Resonator-Type Case ......................................42

3.2.3.1.4 N Distinct Resonator-Type Case ...............................44

3.2.3.2 Resonator Sequence Variation Case ..........................................46

3.2.3.3 Material Variation Case .............................................................48

3.3 Summary....

IV. IMPACT ENERGY HARVESTING USING ASSONANT ACOUSTIC

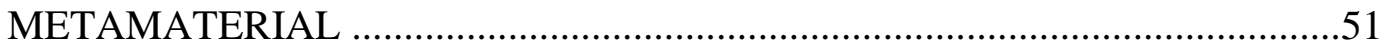

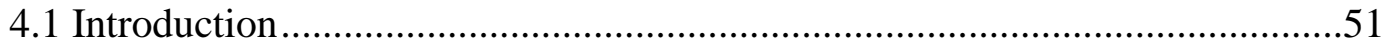

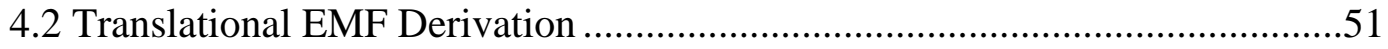

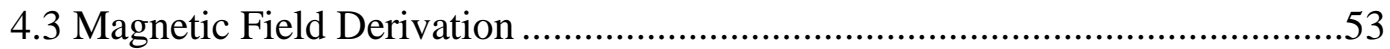

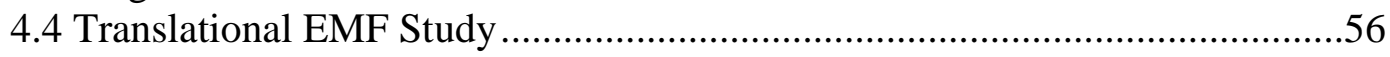

4.4.1 Definition of Test Cases..................................................................59

4.4.1.1 Theoretical Model ........................................................59

4.4.1.2 Semi-analytical Model ....................................................60

4.4.1.2 Experimental Model............................................................62

4.4.2 Experimental Model.........................................................................60

4.4.3 Definition of Test Cases................................................................62

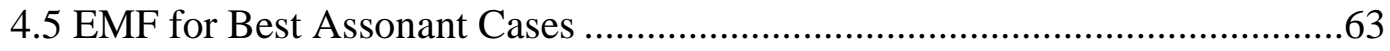

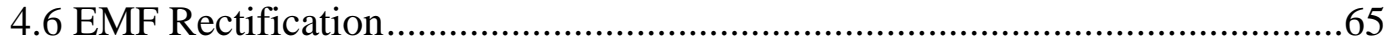

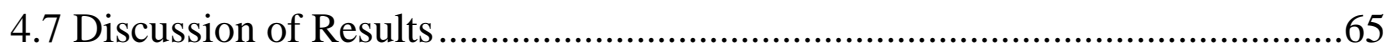

4.7.1 Magnetic Field Plots ......................................................................66

4.7.2 Translational EMF Verification ..................................................68

4.7.2.1 EMF Results for Co-located Configuration ...............................68

4.7.2.2 EMF Results for Mirror Configuration......................................70

4.7.2.3 EMF Results for Interspersed Configuration ............................72

4.7.3 EMF for Best Assonant Cases ..........................................................73

4.7.3.1 Best Resonant-Type Variation Case ........................................74

4.7.3.2 Best Resonant Sequence Variation Case ....................................75

4.7.3.3 Best Material Variation Case .......................................................... 77

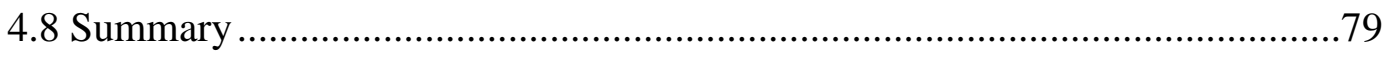

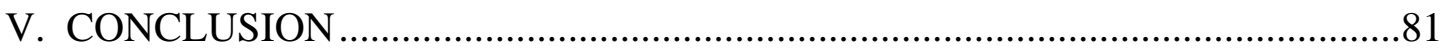

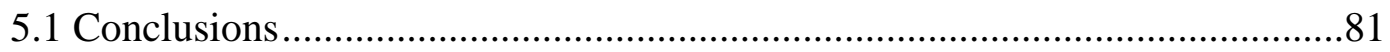

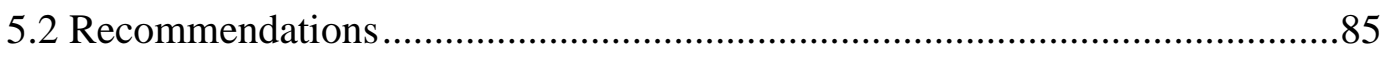

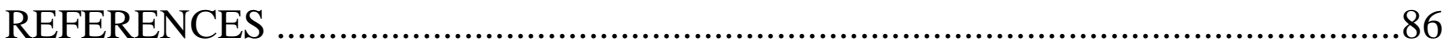

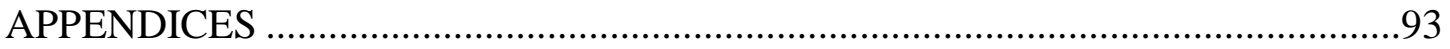


APPENDIX A: Python and MATLAB Codes .98 APPENDIX B: Additional Figures and Plots . 


\section{LIST OF TABLES}

\section{Table}

2.1 Summary of base parameters for LRAM 16

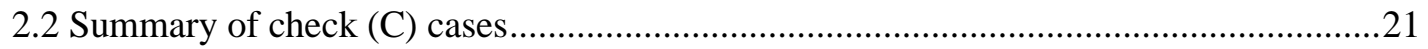

2.3 Summary of overall baseline case for comparison to all AMB cases ...........................22

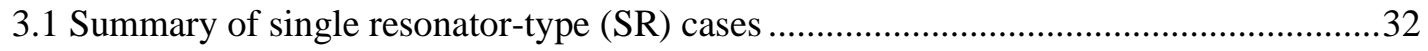

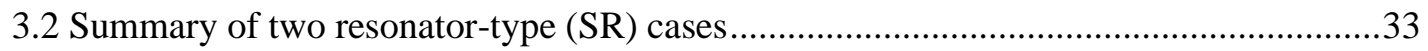

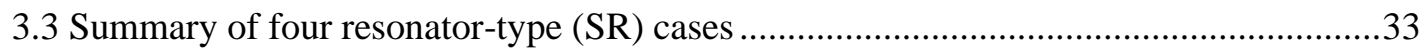

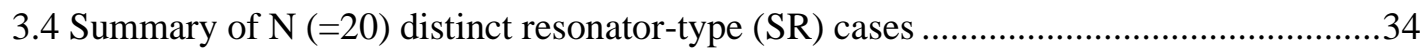

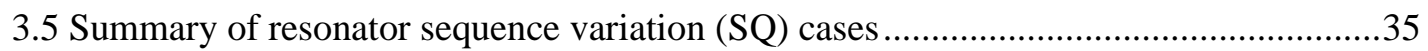

3.6 Summary of host material stiffness variation (SM) cases................................................

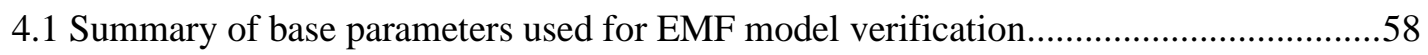

4.2 Summary of test case studies for the proposed theoretical model.................................59

4.3 Summary of test case studies for the proposed semi-analytical model..........................61

4.4 Summary of test case studies for the proposed experimental model .............................62

4.5 Summary of resonator configuration for best assonant cases ......................................62

4.6 Summary of magnet properties used for EMF study for best assonant cases .................65

4.7 Summary of $\mathrm{V}_{\mathrm{RMS}}$ and percentage error data for theoretical (TCo) and semi-analytical

(SCo) co-located coil configuration ...................................................................... 70

4.8 Summary of $\mathrm{V}_{\mathrm{RMS}}$ and percentage error data for theoretical (TMC) and semi-analytical

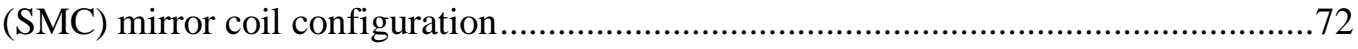

4.9 Summary of $\mathrm{V}_{\mathrm{RMS}}$ and percentage error data for theoretical (TIC) and semi-analytical

(SIC) interspersed coil configuration

4.10 Summary of harvested voltage RMS for best resonator-type variation case (SR14)

4.11 Summary of harvested voltage RMS data for best resonator sequence case (SQ6) 
4.12 Summary of harvested voltage RMS data for best material variation case (SM6) 


\section{LIST OF FIGURES}

Figure $\quad$ Page

1.1 Collapse of the Tacoma Narrows Bridge, Washington state, 1940 [64] ..........................

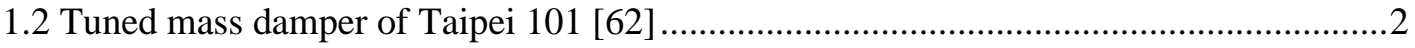

1.3 A one-dimensional mass-in-mass lattice structure [10] ...............................................

1.4 Nondimensionalized dispersion curves of a mass-in-mass lattice model [10] ...............6

1.5 Microstructure of mass-in-mass unit model and effective mass model..........................8

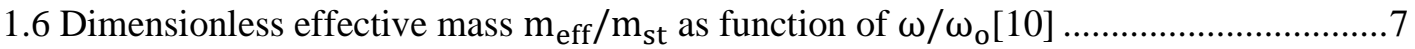

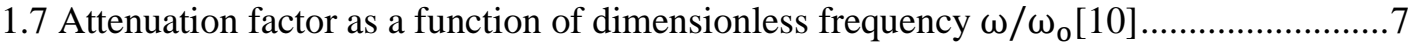

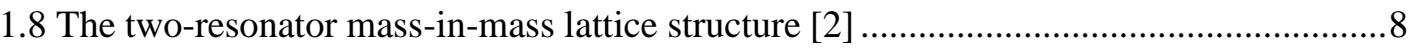

1.9 Nondimensionalized dispersion curves for two resonator mass-in-mass systems [2] ......9

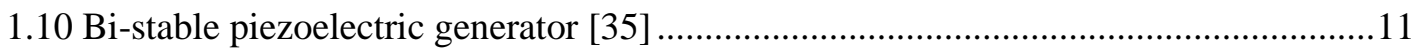

1.11 Schematic for multifunctional array of resonators [36] ............................................ 12

1.12 Schematic depiction of an acoustic metamaterial barrier with $\mathrm{N}$ unit-cells..................13

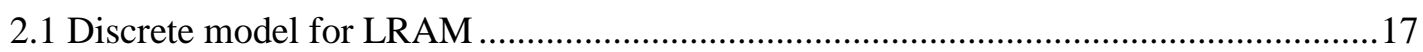

2.2 Dispersion curves and bandgap frequency range for an LRAM with a local resonance

frequency of $500 \mathrm{~Hz}$

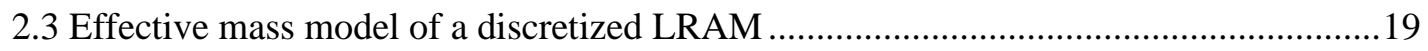

2.4 Normalized effective mass vs. normalized effective excitation frequency.....................20

2.5 Simulation model for a one-dimensional finite length AMB with $\mathrm{N}(=20)$ units ...........21

2.6 (a) Displacement time history and (b) frequency spectrum for the step input excitation22

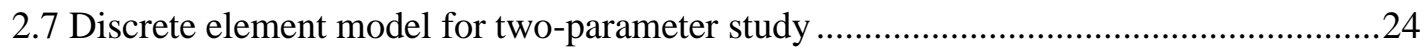

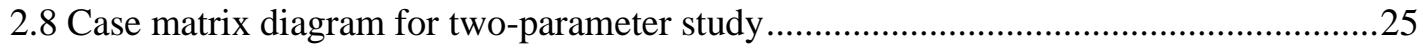

2.9 Output displacement time histories for check cases ....................................................26

2.10 Output displacement frequency spectra for check cases ............................................27

2.11 Input and output displacement time histories for the baseline (SR7) case...................28

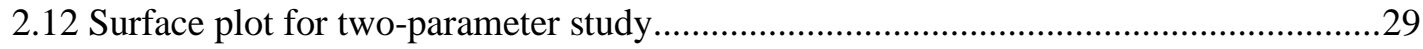


Figure

3.1 Input, baseline (SR7) and output displacement time histories for the single resonator-type cases 38

3.2 Input, baseline (SR7) and output displacement frequency spectra for the single resonatortype cases

3.3 Output displacement $\left(\mathrm{D}^{*}\right)$ and power spectral $\left(\mathrm{S}^{*}\right)$ transmissibility and normalized wave speed $\left(\mathrm{C}^{*}\right)$ for the single resonator-type cases

3.4 Input, baseline (SR7) and output displacement time histories for the two resonator-type cases

3.5 Input, baseline (SR7) and output displacement frequency spectra for the two resonator-type cases

3.6 Output displacement $\left(\mathrm{D}^{*}\right)$ and power spectral $\left(\mathrm{S}^{*}\right)$ transmissibility and normalized wave speed $\left(\mathrm{C}^{*}\right)$ for the two resonator-type cases

3.7 Input, baseline (SR7) and output displacement time histories for the four resonator-type cases

3.8 Input, baseline (SR7) and output displacement frequency spectra for the four resonator-type cases

3.9 Output displacement ( $\left.\mathrm{D}^{*}\right)$ and power spectral $\left(\mathrm{S}^{*}\right)$ transmissibility and normalized wave speed $\left(\mathrm{C}^{*}\right)$ for the four resonator-type cases

3.10 Input, baseline (SR7) and output displacement time histories for the $\mathrm{N}(=20)$ distinct resonator-type cases

3.11 Input, baseline (SR7) and output displacement frequency spectra for the $\mathrm{N}(=20)$ distinct resonator-type cases

3.12 Output displacement $\left(\mathrm{D}^{*}\right)$ and power spectral $\left(\mathrm{S}^{*}\right)$ transmissibility and normalized wave speed $\left(\mathrm{C}^{*}\right)$ for the four resonator-type cases

3.13 Input, baseline (SR7) and output displacement time histories for the resonator sequence variation cases 46

3.14 Input, baseline (SR7) and output displacement frequency spectra for resonator sequence variation cases

3.15 Output displacement ( $\left.\mathrm{D}^{*}\right)$ and power spectral $\left(\mathrm{S}^{*}\right)$ transmissibility and normalized wave speed $\left(\mathrm{C}^{*}\right)$ for the resonator sequence variation cases.

3.16 Input, baseline (SR7) and output displacement time histories for the host material stiffness variation cases. 
Figure

3.17 Input, baseline (SR7) and output displacement frequency spectra for the host material stiffness variation cases.

3.18 Output displacement $\left(\mathrm{D}^{*}\right)$ and power spectral $\left(\mathrm{S}^{*}\right)$ transmissibility and normalized wave speed $\left(C^{*}\right)$ for the host material stiffness variation cases

4.1 A simple depiction of an oscillating magnet along the axial direction surrounded by coils

4.2 Schematic depiction of a finite magnetized cylinder

4.3 Exploded view of the harvesting device

4.4 Schematic depiction for (a) co-located, (b) mirror and (c) interspersed configuration...59

4.5 Spring-mass model representation of the electromechanical coupling ..........................59

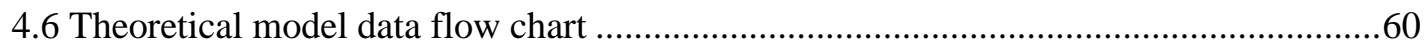

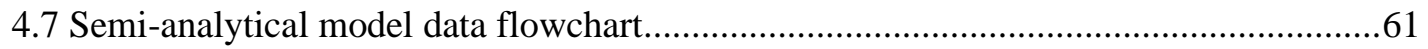

4.8 Schematic of, (a) experimental model (b) image from experiment setup, (c) close-

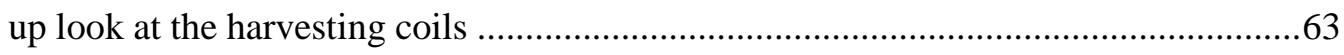

4.9 A schematic for AM harvester cells connected in series ..............................................61

4.10 Surface plot of the magnetic field in the axial direction ..............................................66

4.11 Surface plot of the magnetic field in the radial direction .............................................66

4.12 Magnetic field lines of a Neodymium magnet.........................................................67

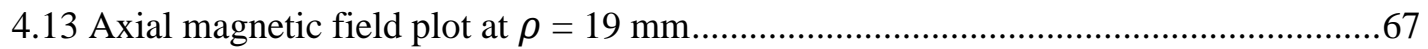

4.14 EMF data, (a) time history and (b) frequency spectra plots of $52 \mathrm{~Hz}$ excitation input with co-located coil configuration

4.15 EMF data, (a) time history and (b) frequency spectra plots of $54 \mathrm{~Hz}$ excitation input with co-located coil configuration

4.16 EMF data, (a) time history and (b) frequency spectra plots of $52 \mathrm{~Hz}$ excitation input with mirror coil configuration

4.17 EMF data, (a) time history and (b) frequency spectra plots of $54 \mathrm{~Hz}$ excitation input with mirror coil configuration

4.18 EMF data, (a) time history and (b) frequency spectra plots of $52 \mathrm{~Hz}$ excitation input with interspersed coil configuration 
Figure

4.19 EMF data, (a) time history and (b) frequency spectra plots of $54 \mathrm{~Hz}$ excitation input with interspersed coil configuration

4.20 Rectified EMF (a) time history and (b) frequency spectrum plot for best resonator-type variation case (SR14)

4.21 Rectified EMF (a) time history and (b) frequency spectrum plot for best resonator sequence case (SQ6)

4.22 Rectified EMF (a) time history and (b) frequency spectrum plot for best material variation case (SM6)

B.1.1 EMF data, (a) time history and (b) frequency spectra plots for $56 \mathrm{~Hz}$ excitation input with co-located coil configuration.

B.1.2 EMF data, (a) time history and (b) frequency spectra plots for $58 \mathrm{~Hz}$ excitation input with co-located coil configuration

B.2.1 EMF data, (a) time history and (b) frequency spectra plots for $56 \mathrm{~Hz}$ excitation input with mirror coil configuration

B.2.2 EMF data, (a) time history and (b) frequency spectra plots for $58 \mathrm{~Hz}$ excitation input with mirror coil configuration

B.3.1 EMF data, (a) time history and (b) frequency spectra plots for $56 \mathrm{~Hz}$ excitation input with interspersed coil configuration

B.3.2 EMF data, (a) time history and (b) frequency spectra plots for $58 \mathrm{~Hz}$ excitation input with interspersed coil configuration

B.4.1 Theoretical input displacement (a) time history and (b) frequency spectra for $52 \mathrm{~Hz}$ excitation

B.4.2 Experimental input displacement (a) time history and (b) frequency spectra for $52 \mathrm{~Hz}$ excitation

B.4.3 Theoretical response displacement (a) time history and (b) frequency spectra for $52 \mathrm{~Hz}$ excitation

B.4.4 Experimental response displacement (a) time history and (b) frequency spectra for $52 \mathrm{~Hz}$ excitation

B.5.1 Theoretical input displacement (a) time history and (b) frequency spectra for $54 \mathrm{~Hz}$ excitation 
B.5.2 Experimental input displacement (a) time history and (b) frequency spectra for

$54 \mathrm{~Hz}$ excitation

B.5.3 Theoretical response displacement (a) time history and (b) frequency spectra for

$54 \mathrm{~Hz}$ excitation

B.5.4 Experimental response displacement (a) time history and (b) frequency spectra for $54 \mathrm{~Hz}$ excitation

B.6.1 Theoretical input displacement (a) time history and (b) frequency spectra for $56 \mathrm{~Hz}$ excitation

B.6.2 Experimental input displacement (a) time history and (b) frequency spectra for $56 \mathrm{~Hz}$ excitation

B.6.3 Theoretical response displacement (a) time history and (b) frequency spectra for $56 \mathrm{~Hz}$ excitation

B.6.4 Experimental response displacement (a) time history and (b) frequency spectra for $56 \mathrm{~Hz}$ excitation

B.7.1 Theoretical input displacement (a) time history and (b) frequency spectra for

$58 \mathrm{~Hz}$ excitation

B.7.2 Experimental input displacement (a) time history and (b) frequency spectra for $58 \mathrm{~Hz}$ excitation

B.7.3 Theoretical response displacement (a) time history and (b) frequency spectra for

$58 \mathrm{~Hz}$ excitation

B.7.4 Experimental response displacement (a) time history and (b) frequency spectra for $58 \mathrm{~Hz}$ excitation

B.8.1 Displacement response (a) time history and (b) frequency spectra for $\mathrm{SR} 14,100 \mathrm{~Hz}$ resonator sets $(6 \times 100 \mathrm{~Hz})$

B.8.2 Displacement response (a) time history and (b) frequency spectra for $\mathrm{SR} 14,300 \mathrm{~Hz}$ resonator sets $(6 \times 300 \mathrm{~Hz})$

B.8.3 Displacement response (a) time history and (b) frequency spectra for $\mathrm{SR} 14,700 \mathrm{~Hz}$ resonator sets $(5 \times 700 \mathrm{~Hz})$

B.8.4 Displacement response (a) time history and (b) frequency spectra for SR14, $1500 \mathrm{~Hz}$ resonator sets $(3 \times 1500 \mathrm{~Hz})$

B.8.5 Harvested EMF (a) time history and (b) frequency spectra for SR14, 100Hz resonator sets $(6 \times 100 \mathrm{~Hz})$ 
Figure

B.8.6 Harvested EMF (a) time history and (b) frequency spectra for SR 14, 300Hz resonator sets $(6 \times 300 \mathrm{~Hz})$

B.8.7 Harvested EMF (a) time history and (b) frequency spectra for SR14, 700Hz resonator sets $(5 \times 700 \mathrm{~Hz})$

B.8.8 Harvested EMF (a) time history and (b) frequency spectra for SR14, $1500 \mathrm{~Hz}$ resonator sets $(3 \times 1500 \mathrm{~Hz})$

B.9.1 Displacement response (a) time history and (b) frequency spectra for SQ6, $100 \mathrm{~Hz}$ resonator sets $(6 \mathrm{x} 100 \mathrm{~Hz})$

B.9.2 Displacement response (a) time history and (b) frequency spectra for SQ6, $300 \mathrm{~Hz}$ resonator sets $(6 \times 300 \mathrm{~Hz})$

B.9.3 Displacement response (a) time history and (b) frequency spectra for SQ6, $700 \mathrm{~Hz}$ resonator sets $(5 \times 700 \mathrm{~Hz})$

B.9.4 Displacement response (a) time history and (b) frequency spectra for SQ6, $1500 \mathrm{~Hz}$ resonator sets $(3 \times 1500 \mathrm{~Hz})$

B.9.5 Harvested EMF (a) time history and (b) frequency spectra for SQ6, $100 \mathrm{~Hz}$ resonator sets $(6 \mathrm{x} 100 \mathrm{~Hz})$

B.9.6 Harvested EMF (a) time history and (b) frequency spectra for SQ6, $300 \mathrm{~Hz}$ resonator sets $(6 \times 300 \mathrm{~Hz})$

B.9.7 Harvested EMF (a) time history and (b) frequency spectra for SQ6, 700Hz resonator sets $(5 \times 700 \mathrm{~Hz})$

B.9.8 Harvested EMF (a) time history and (b) frequency spectra for SQ6, $1500 \mathrm{~Hz}$ resonator sets $(3 \times 1500 \mathrm{~Hz})$

B.10.1 Displacement response (a) time history and (b) frequency spectra for SM6, $100 \mathrm{~Hz}$ resonator sets $(6 \mathrm{x} 100 \mathrm{~Hz})$

B.10.2 Displacement response (a) time history and (b) frequency spectra for SM6, $300 \mathrm{~Hz}$ resonator sets $(6 \times 300 \mathrm{~Hz})$

B.10.3 Displacement response (a) time history and (b) frequency spectra for SM6, $700 \mathrm{~Hz}$ resonator sets $(5 \times 700 \mathrm{~Hz})$

B.10.4 Displacement response (a) time history and (b) frequency spectra for SM6, $1500 \mathrm{~Hz}$ resonator sets $(3 \times 1500 \mathrm{~Hz})$

B.10.5 Harvested EMF (a) time history and (b) frequency spectra for SM6, 100Hz resonator sets $(6 \mathrm{x} 100 \mathrm{~Hz})$ 
Figure

Page

B.10.6 Harvested EMF (a) time history and (b) frequency spectra for SM6, $300 \mathrm{~Hz}$ resonator sets $(6 \times 300 \mathrm{~Hz})$ 126

B.10.7 Harvested EMF (a) time history and (b) frequency spectra for SM6, 700Hz resonator sets $(5 \times 700 \mathrm{~Hz})$ .126

B.10.8 Harvested EMF (a) time history and (b) frequency spectra for SM6, 1500Hz resonator sets $(3 \times 1500 \mathrm{~Hz})$ 126 


\section{CHAPTER I}

\section{INTRODUCTION}

\section{$\underline{1.1 \text { Introduction and Motivation }}$}

In many industrial applications, impacts, shocks, and vibrations can cause undesirable

effects primarily for engineered systems, which may lead to safety and efficacy issues. Impact occurs when two or more bodies collide with a relatively large forces in a short time period. Many mechanical systems are subject to various types of shocks and vibrations. For instance, in the automotive and aerospace industry, shocks or vibrations occurs due to the dynamic forces generated by various mechanical devices like compressors, pumps, engines, aircraft wings, tires, etc. Similarly, in civil structures, it can be caused by earthquakes, tsunamis, wind, etc.

Shocks and vibrations have caused multiple catastrophic failures in human-made structures. One of the most notable accidents is, resonant vibrations destroying the Tacoma Narrows Bridge, videos taken of the disaster show the deck rolling up and down and twisting these motions wildly. The incident was especially surprising at the time as the structure was designed to withstand winds of up to 120 miles per hour, however, the structure collapsed in just a 40 mile per hour traveling wind [64]. The wind was at just the right speed and striking angle to set off the vibrations; continued winds increased the oscillations until the waves grew so large and violently destroying the bridge. This harmonic vibration between the wind and the bridge is known as resonance. 


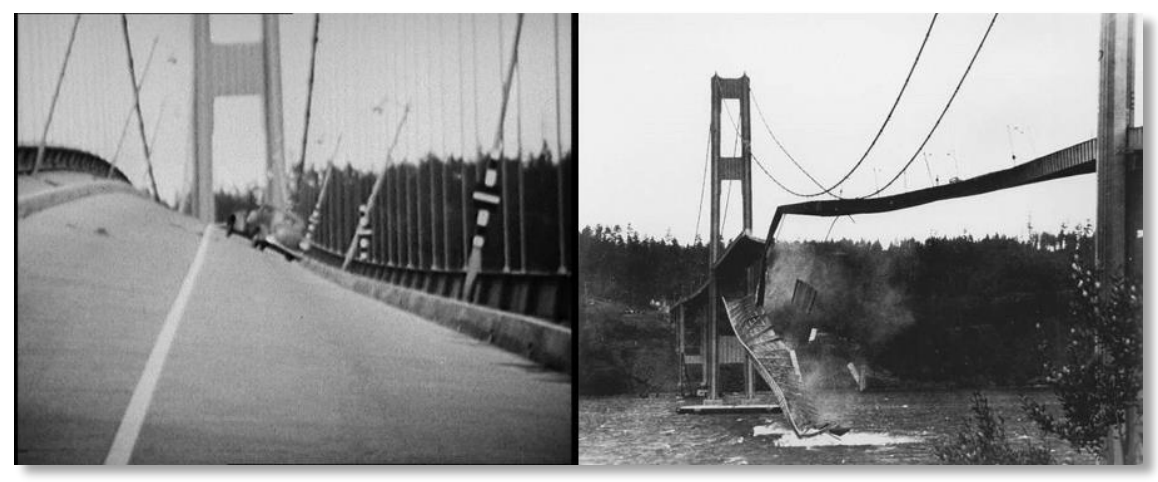

Figure 1.1: Collapse of the Tacoma Narrows Bridge, Washington state, 1940 [64].

To avoid undesirable dynamic loadings on a structure, engineers incorporate absorbers into their design to interrupt the harmonic waves and prevent them from growing. Most common impact mitigation methods can be classified under active and passive systems. Active isolation systems utilize sensors and actuators that produce disruptive interference to cancel out incoming vibration. Passive isolators make use of materials and mechanical linkages that absorb and damp the mechanical waves.

Active isolation systems were being developed as early as the 1950s, they were widely used in the automotive and aerospace industry. They are many reasons which explains why they have been slow to develop into wider use. Most active vibrations isolation systems are relatively complex, costly and often provide only slight improvements in performance compared to conventional passive vibration isolations techniques. The complexity of active isolation systems in many instances have caused challenges of installation and continued maintenance, moreover, those systems require power input.

The application of passive isolators designed to mitigate shocks have seen an increased interest in many engineering fields. Passive systems do not require external energy source instead they can be designed to convert the energy generated by a particular impact into some other form 
of energy. Common passive shock absorbers are, pneumatic/air dampers, mechanical springs, tuned mass dampers, rubber/foams, etc. Figure 1.2 illustrates an engineering marvel to designed to limit the vibration of a 500-meter-long building located in Taipei City, Taiwan, currently it is known to be the world's largest and heaviest tuned mass damper.

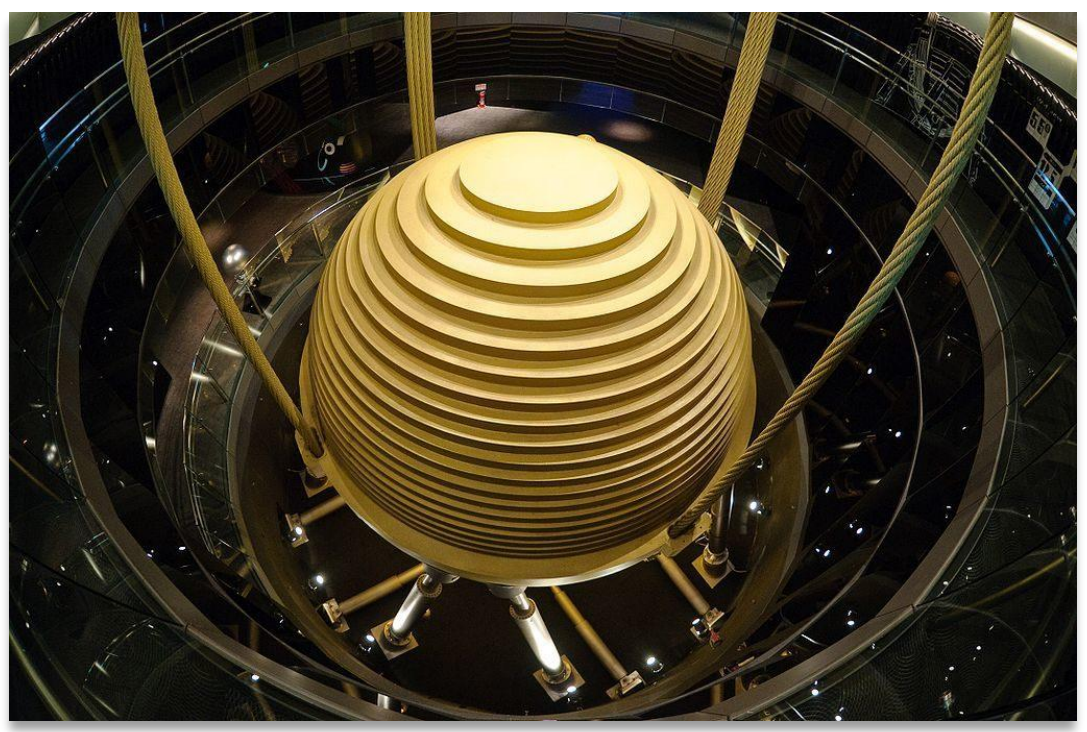

Figure 1.2: Tuned mass damper of Taipei 101 [62].

Impact loadings incident on an elastic media are known to generate broadband stress waves within the given structure. Most conventional active and passive vibration isolators are only effective for a narrowband frequency range. However, there are several ways to mitigate waves with broadband frequencies. Recently many researches have shown that acoustic metamaterials (AM) are known to be effective in manipulating acoustic and mechanical waves. AM are artificially engineered structural materials that posses' unique dynamic effective properties, not commonly found in nature. AM's designed with multi-resonator configuration have shown effectiveness in mitigating waves generated by an impact-type loading. From a materials perspective it might be of interest to investigate a design framework that can be used to effectively study the interactive mechanisms between the local resonators and host structure. 


\subsection{Literature Review}

In recent years, metamaterials as a class of engineered materials have received vast interest from researchers and scientists. Metamaterials are artificial materials engineered to have unusual optical, mechanical or thermal properties not found in nature $[4,5,6]$, the concept was initially explored in the field of optics where it was used to manipulate electromagnetic waves; this is often made conceivable by fine-tuning the permittivity and permeability of the electromagnetic fields to give a response with a negative refractive index. The idea of negative refraction in an electromagnetic wave was proposed by Veselago [4]. In photonic metamaterials (PM), subwavelength engineered elements supplant existing atoms as the dominating determinant of electromagnetic properties; due to this reason fabricating a PM is difficult as the structural elements must be much smaller than the wavelength of light. The first AM model was realized by embedding rubber-coated lead spheres (resonator) in an epoxy matrix (host material) to capture dipolar resonances; an anomalous bandgap $(400-600 \mathrm{~Hz})$ was observed for this design [1], and all frequencies operating within the bandgap region are shown to be mitigated.

AM's acquire their unique dynamic properties from the locally engineered feature microstructures that show uncharacteristic elastic wave propagation. Depending on their scalability, microstructure in the context of AM is used as a terminology to refer to engineered local structures and can even be macroscopic [7]. For acoustic waves, bulk modulus and density determine the characteristic of wave propagation. The theoretical possibility of having negative mass density and modulus in AM were explored $[1,8]$.

Liu and Chan illustrated that the mass density and bulk modulus could be made negative simultaneously by dispersing soft rubber in water [9]. It was also demonstrated that the effective mass could be negative for particular frequency ranges $[1,10,11]$. Negative bulk modulus has been 
studied by Fang et al. using a cluster of Helmholtz resonators [12], conjointly, the negative mass was realized using a mass-in-mass structure [13]. The practical application of these systems includes mechanical filters, sound and vibration isolators, and acoustic waveguides.

One of the most appealing features in acoustic metamaterials is the possibility to alter the desired bandgap to restrain the propagation of waves for specific frequencies. Numerous amounts of theoretical and experimental studies have been made on the bandgaps of acoustic and elastic wave propagation in AM. Kushwaha et al. [14] demonstrated one of the first analytical studies on simple periodic structure; however, the study lacked to show the band structures for a mixed longitudinal-transverse mode. The complete acoustic bandgaps that are independent of polarizations were done by Martinsson and Movchan [15]. It is also well known that the attenuation bandwidth can be extended by utilizing multiple resonators, for which the internal resonances may be customized to cover a range of frequencies that extends beyond that achievable with one resonator. As an initial trial, a metamaterial consisting of two resonators was proposed and studied for the demonstration of multiple bandgap generation [2].

An interesting observed feature in AM is the presence of Bragg scattering, caused by destructive interference of waves [16]. AM can be designed to manipulate sonic waves such as, refraction like beam bending and focusing [17,18]. Different from Bragg scattering, AM's can control acoustic waves that are much smaller than the wavelength of the propagating wave, which fundamentally implies the introduction of a bandgap at a much lower frequency $[9,18]$. The negative effective mass density in AM arises from the out-of-phase movement between the unit cell and local attachment.

The locally resonant lattice model was first introduced to be used as a mechanical filter by Vincent [19], he demonstrated by using a loaded spiral spring wound in one piece on a surface of a cylinder with local attachment installed by a pair of hooks. The dispersion relation for an LRAM 
can be derived by modeling it as a one-dimensional infinite mass-in-mass lattice system as shown in Figure 1.3 [10].

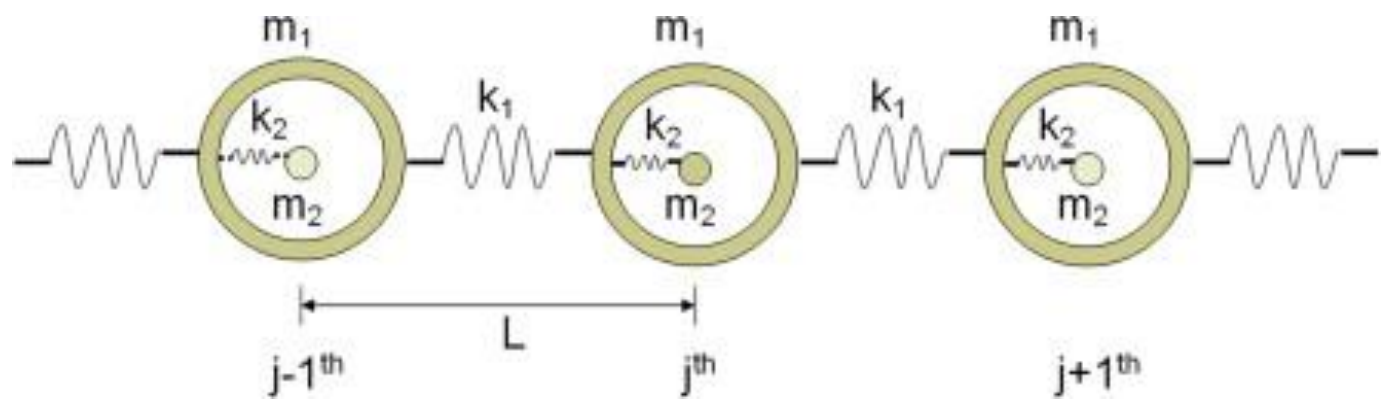

Figure 1.3: A one-dimensional mass-in-mass lattice structure [10].

Where, $m_{1}$ and $m_{2}$ are the mass of the host material and resonator, respectively. The stiffness of the inclusion is represented by $k_{2}$. The natural frequency of the resonator, $\omega_{o}{ }^{2}=\frac{k_{2}}{m_{2}}$.

Figure 1.4 shows the nondimensionalized dispersion curves of a mass-in-mass lattice model [10]. For a specific set of material constants chosen, a bandgap behavior as depicted in Figure 1.4 could be realized.

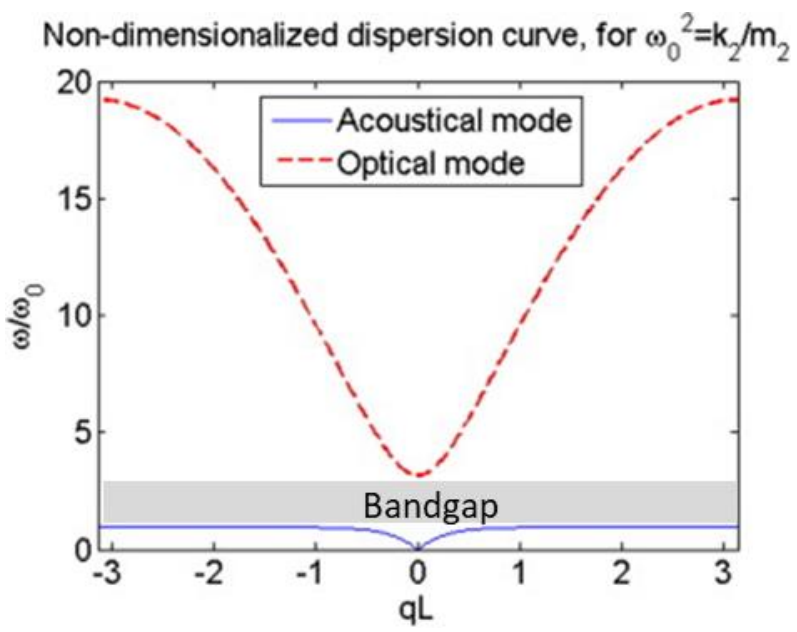

Figure 1.4: Nondimensionalized dispersion curves of a mass-in-mass lattice model [10]. 
Yao et al. [20] illustrated the negative effective mass on the dynamic transmission using a one-dimensional spring-mass system. The lowest branch curve is called the acoustic wave mode, which is dictated by the local resonance frequency. The higher branch, optical mode arises from the periodicity of the lattice structure. In the region where the bandgap exists as seen in Figure 1.4 there will be no wave propagation as the effective mass becomes purely imaginary.

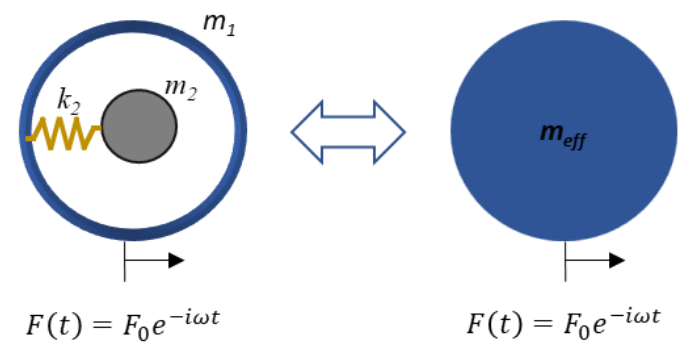

Figure 1.5: Microstructure of mass-in-mass unit model and effective mass model.

It is necessary to understand the practical meaning of negative effective mass density, and the bandgaps associated with it, a mass-in-mass lattice model of a single resonator was studied by Huang et al. [10]. By equating the motion of a mass-in-mass unit model with an equivalent effective model, as seen in Figure 1.5, the equation for effective mass is derived as:

$$
\begin{gathered}
m_{e f f}=m_{1}+\frac{m_{2} k_{2}}{k_{2}-m_{2} \omega^{2}} \\
\omega^{2}=\frac{2 k_{1}(1-\cos (q L))}{m_{e f f}}
\end{gathered}
$$

Where, $\omega$ is excitation frequency and $q$ is wave number. It can be seen from equation 1.1 that the effective mass, $m_{e f f}$ is frequency dependent. At the long wavelength limit $(\omega \rightarrow 0), m_{e f f}$ approaches the static mass, $m_{s t}=m_{1}+m_{2}$. Because the excitation frequency $\omega$ and external stiffness $k_{1}$ can only be positive, therefore, to obtain negative effective mass $1-\cos (q L)$ must be less than zero in other words the dimensionless wave number qL is purely imaginary in this range. 


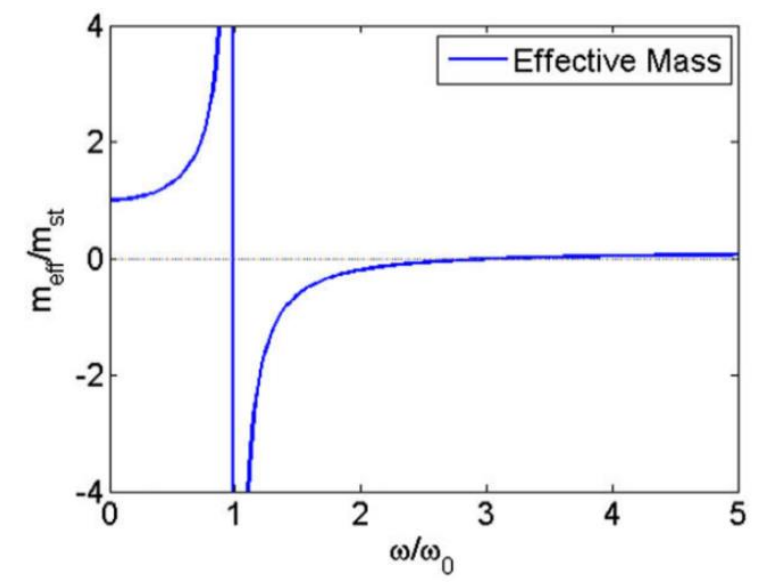

Figure 1.6: Dimensionless effective mass $m_{e f f} / m_{s t}$ as function of $\omega / \omega_{o}[10]$.

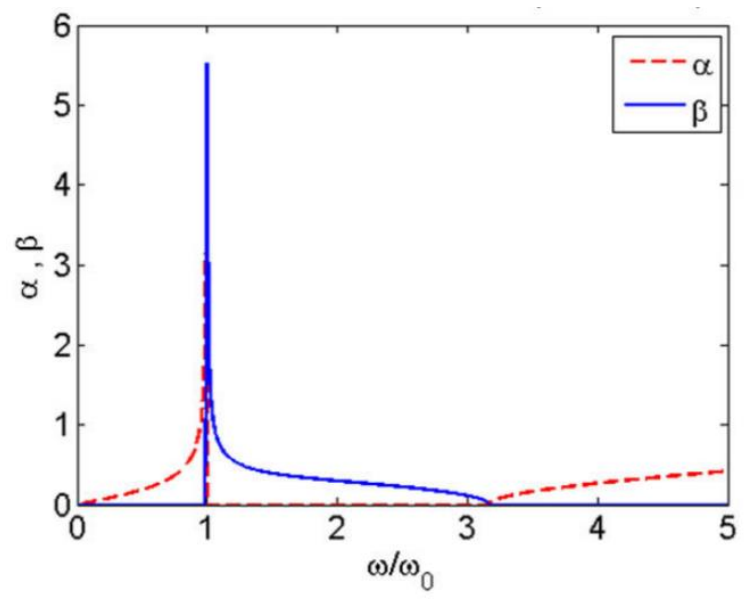

Figure 1.7: Attenuation factor as a function of dimensionless frequency $\omega / \omega_{o}$ [10].

In Figure 1.7 the real and imaginary part of wavenumber are plotted against the normalized excitation frequency. $\alpha$ and $\beta$ represent the real and imaginary part of the normalized wave number. The imaginary part, $\beta$ is known as the attenuation factor. Thus, the frequency range of effectivemass negativity corresponds to a bandgap for harmonic wave propagation. The effective mass model provides a simple means to characterize the longitudinal elastic wave propagation behavior of AM configuration having different local attachments. 
While electromagnetic metamaterials, were the first to be developed and have been successfully employed in several applications [22] to enhance performance, their acoustic counterparts, predominantly the locally resonant variants [23] have seen lesser success being transitioned to applications. Ensuring primary structural functionalities is known to be an issue when it comes to LRAM applications [5], to realize the mechanical wave manipulate capabilities, the engineered features must not compromise the weight, volume, stiffness or strength of the material. Although the locally resonant class of AM present this practical challenge, an optimal design from a wave manipulation point of view are important from an application perspective [21, 24]. There are several studies that show the optimization of the bandgap region where the main focus is on optimizing the characteristics or type of the local features. Kulkarni and Manimala [25], investigated and demonstrated tunable bandgap behavior by using a dynamic mass amplification device called an inerter. This method can effectively tune the bandgap behavior without having to add large permanent mass.

Huang and Sun [2] showed that by having multiple resonators in a mass-in-mass lattice system as shown in Figure 1.8, it is possible to open several band gaps.

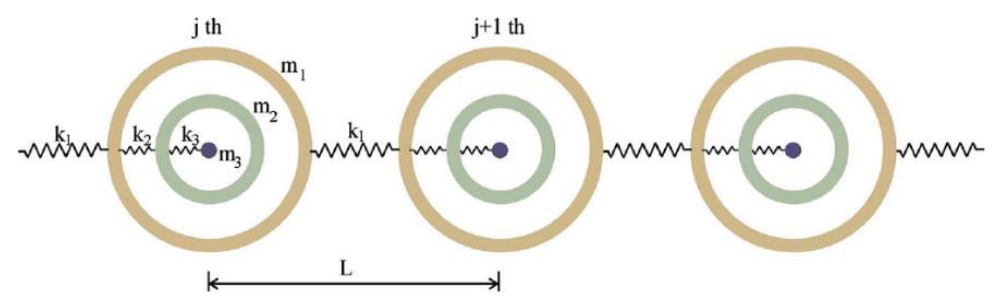

Figure 1.8: The two-resonator mass-in-mass lattice structure [2].

Figure 1.9 shows that an additional bandgap opening as a consequence of having an additional resonator. The bandgaps may also be shifted by changing the mass ratios. If the multiple 
mass lattice system is treated as an effective single mass lattice system, then the effective mass would become negative in the bandgap frequencies.

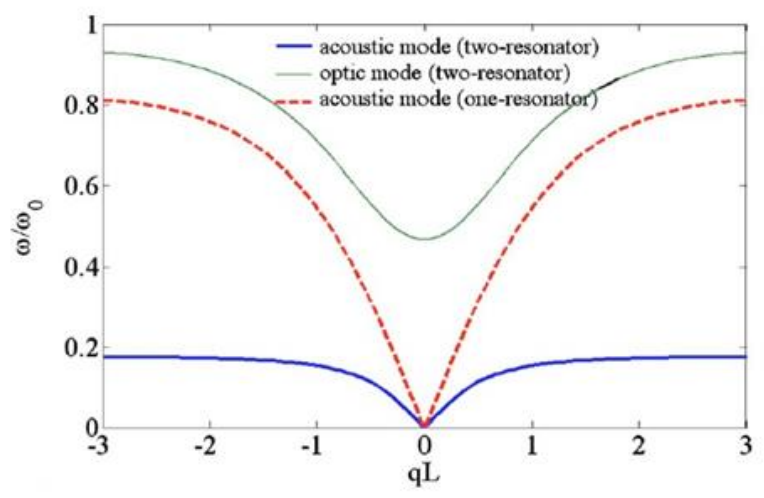

Figure 1.9: Nondimensionalized dispersion curves for two resonator mass-in-mass systems [2].

A dissipative mass-in-mass lattice system with two resonators was also demonstrated for blast-wave mitigation [26]. Fang et al. [27] showed that using nonlinear resonators, the optimization of the bandgap regions can be altered to increase the attenuation performance for ultralow, and ultra-broadband spectrum. A more holistic design approach that considers not just the local features but also the 'background' features of the host material, as well as the interactive mechanisms between them, could aid the extraction of the most out of AM designs under constrained application scenarios.

One of the most attractive features in AM is the possibility to tailor the required band gap to mitigate wave propagation of specific frequencies. With these noted features, it is also possible to apply acoustic metamaterial concepts for shock mitigation on mechanical structures, a shock impact loading generally exhibits a broadband frequency spectrum due to the force being applied over a short period of time. A step function can be used as a mathematical model to represent an impact/shock loading; a step function will result in a broadband spectrum where the maximum 
bandwidth can be calculated by taking the inverse of time of contact. Several theoretical and experimental studies have been made over the years to realize and understand the various applications of acoustic metamaterials to mitigate shocks/impacts for diverse practical implementations. A considerable amount of research has been conducted over the years to alleviate shock loading on a structure by utilizing different materials and structures. Cellular materials have been used in various structural engineering applications due to their powerful energy absorption capability [28]. However, the form of cellular material as a protective shield for high-speed impact is not desirable. Christou et al. [29] demonstrated using a continuum model to study the attenuation properties of foam-specimens encapsulating filler materials under shock loading by developing a fluid-structure interaction. Su et al. [30] introduced a shock mitigation device that reduces the force on a base of a cylinder by having multiple reflections within the shock mitigation device. Grujicic et al. [31] studied and compared the shock mitigation capacity of polyurea when utilized as a helmet suspension-pad material to that of a traditional foam suspension-pad material. The majority of the studies made for shock-mitigation is either using deformation of materials to absorb the energy, which turns out to damage the material or by counteracting the momentum of the incident shock, which requires complex structures.

Moreover, these concepts will fail in the attenuation of a specific or broader range of frequencies. The unique properties of AM's result in the mitigation of stress wave propagation across the acoustic metamaterial barrier (AMB). Tan et al. [32] demonstrated practical ways to design AM's for blast-wave impact mitigation; the study they performed on single and dualresonator shows the attenuation stress waves. They also mentioned that the dual-resonator configuration gives the best attenuation performance.

In recent years, the field of energy harvesting has gained a significant deal of attention due to the ever-increasing desire to produce portable electronics and smart materials. Some known renewable energy sources are flowing water, solar energy, biofuel, waste heat, and ambient 
vibrations. Kinetic energy is commonly available as random vibrations or displacement noise. It is well known that vibrations are potentially suitable for energy harvesting.

The first attempt of a dual functional AM that dictates the control of acoustic absorption and energy harvesting was studied [33]. The ability of AM to sequester acoustic waves in a localized regime can be then used to harvest sound energy by integrating the electrical system efficiently, this can be achieved by utilizing an electromechanical coupling. The investigation of piezoelectric cantilevers into solid lattice structure to harvest energy within the bandgap was demonstrated [34]. Furthermore, it demonstrated that piezoelectric generators could be used to harvest voltage from structural vibrations [35].

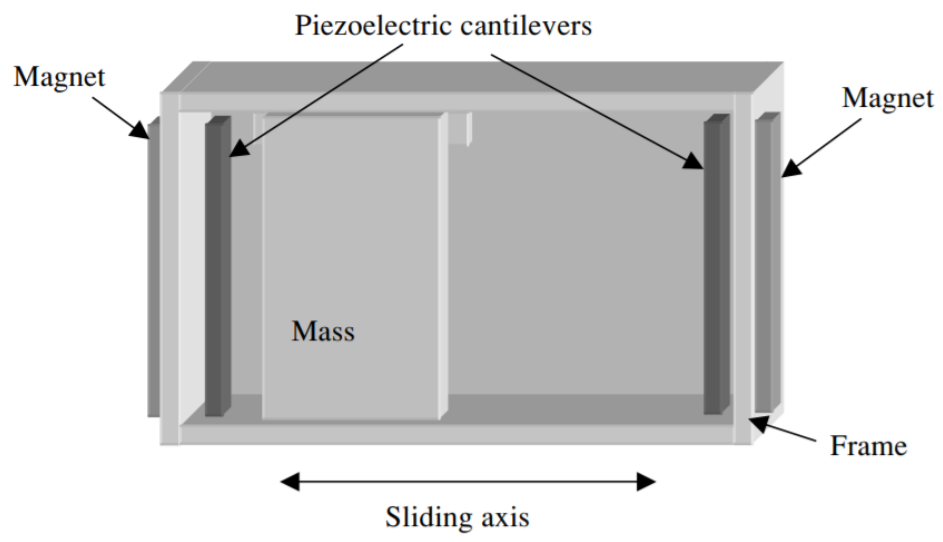

Figure 1.10: Bi-stable piezoelectric generator [35].

Piezoelectric generators are simple to fabricate and can be used for impact-coupled harvesting applications, these generators are subject to direct strain, and therefore their mechanical properties will gradually decline because of fatigue, thus limiting the performance and lifetime.

Carrara et al [37] used a parabolic acoustic mirror to harvest the energy localized in the region of irregularities in aluminum stubs. Most of the current work in acoustic energy harvesting involves the using the kinetic energy concentrated by membrane-type AM [38-40]. An array of resonators to harvest energy with electromagnetic induction were studied [36]. 

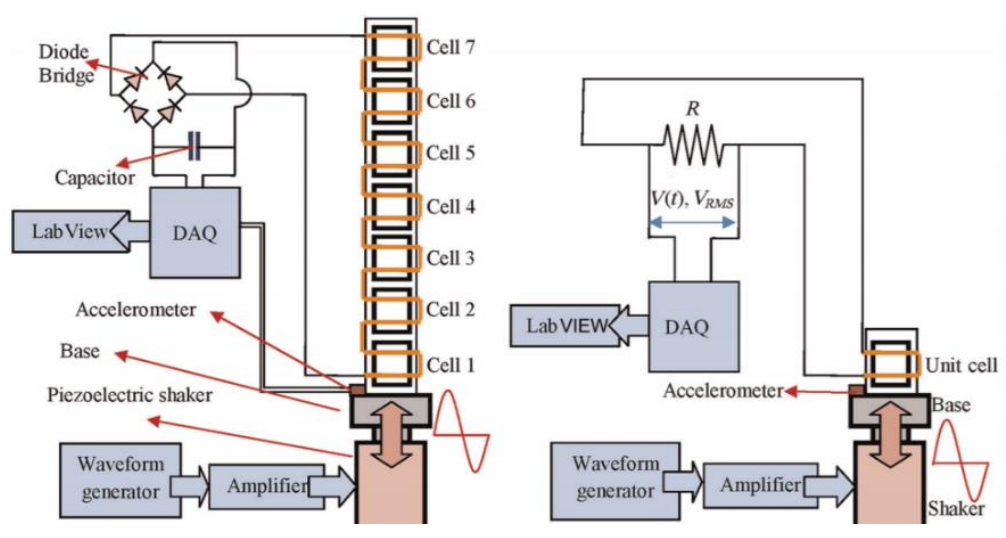

Figure 1.11: Schematic for multifunctional array of resonators [36].

This is done by converting the sound energy to electrical energy. The membranes are calibrated to resonate at the desired frequency. If the ambient sound energy frequency matches that of the membrane, then it is possible to extract energy efficiently.

\subsection{Mechanical Assonance}

This thesis proposes a design of an AMB inspired by a concept called, 'Mechanical Assonance' for impact mitigation and energy harvesting. Mechanical Assonance is hypothesized as a design framework that makes use of not just locally resonant elements but interactive mechanisms between them as well as the host material for an AMB as depicted in Figure 1.10. 


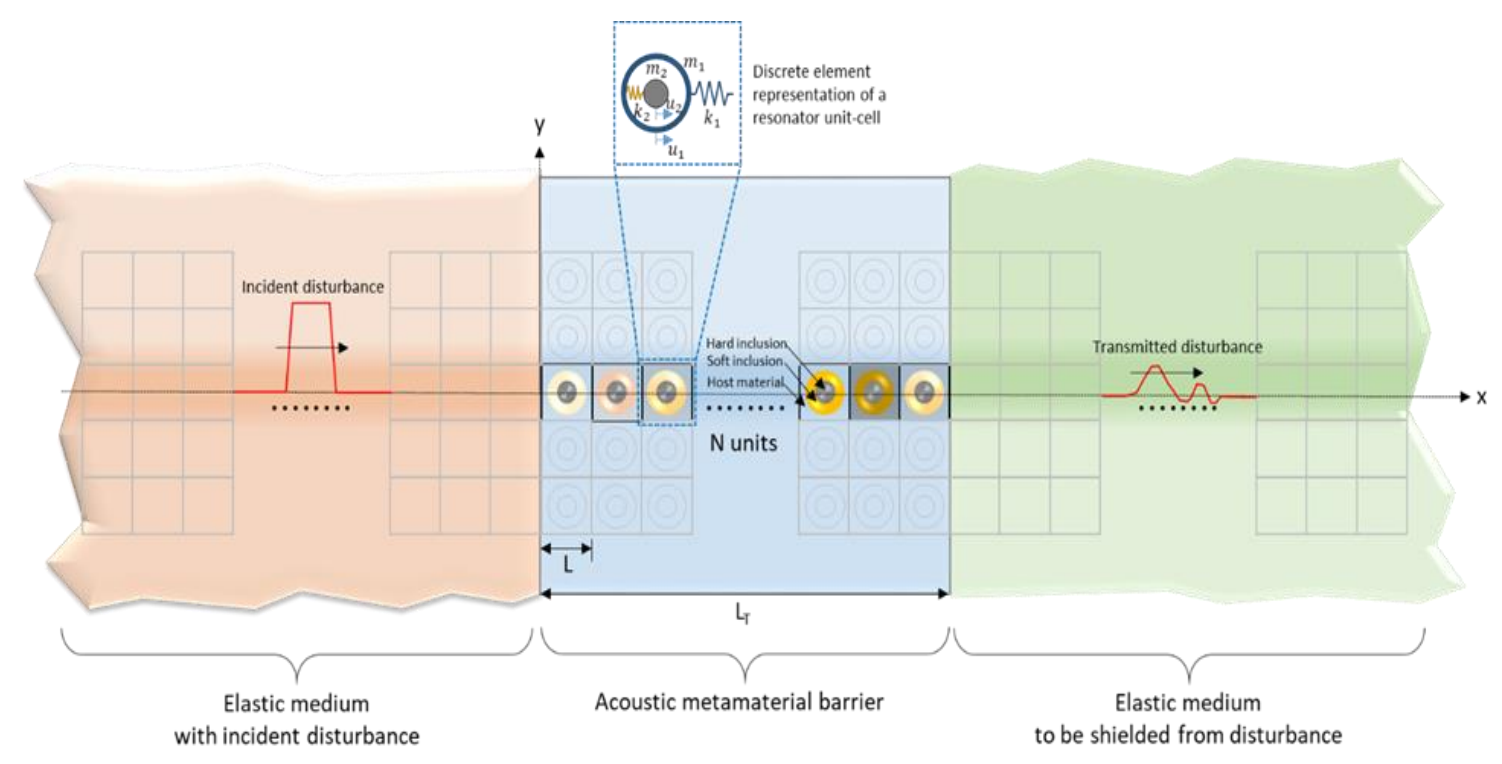

Figure 1.12: Schematic depiction of an acoustic metamaterial barrier with $N$ unit-cells.

For a custom-fitted arrangement of resonators encased inside a host material, it can be feasible to dictate the overall wave propagation by the influence of preceding resonators on the range experienced by succeeding resonators. It could be interesting to investigate resonator modules that provide specific transformational mapping for mechanical waves. Furthermore, the application of this dual functionality is possible since the structure resonance usually causes the enhancement and localization of the deformation energy, which can be readily retrieved with energy harvesters.

The idea of mechanical assonance stems from the literary device called 'assonance' which is used in poetry and prose [65] to confer an internal rhythm and overall lyrical quality to verses to lend a specific mood to them. For instance, the verse, '...A host, of golden daffodils...' from William Wordsworth's famous poem, 'I Wandered Lonely as a Cloud' [63] invokes a lyrical effect by the sequencing of the vowel o's characteristic sound in the words 'host,' 'golden' and 'daffodils.' The Oxford dictionary [66] defines assonance as 'the repetition of the sound of a vowel or diphthong in non-rhyming stressed syllables near enough to each other for the echo to be 
discernible.' In the same vein, the term 'mechanical assonance' may be attributed to the dynamic phenomenon realized by positioning resonators with tuned responses within an engineered waveguide to enforce a wave transformation across it.

From this analogy, one might assume the resonators to be associated with various vowels with the host materials playing the role on consonants such that the overall effect is a function of not just their sounds but also their relative sequencing. The interactive mechanisms between these properties that provide specified outcomes could thus be considered as an assonant mechanism. In this context, one might consider mechanical assonance as the phenomenon of realizing a specific mechanical wave manipulation outcome through a collection of mechanisms related to resonances.

\subsection{Objectives}

The main objectives of this thesis are to:

- Explore parametric dependence for mechanical assonance through a numerical study.

- Conduct numerical optimization for an acoustic metamaterial-inspired barrier for impact mitigation using a design case study.

- Develop a multiphysical model for impact energy harvesting using the acoustic metamaterial-inspired barrier and verify it using proof-of-concept experiments.

\subsection{Chapter Overviews}

The first chapter discusses this study's motivation, which entails the need to conduct this research for a dual functional acoustic metamaterial barrier. It explains the use of AM, the need for optimization for LRAM, and energy harvesting using AM.

In Chapter 2, a theoretical and simulation model for the AMB with defined base parameters and boundary conditions is presented. Check case studies, baseline AMB model, and metrics are 
introduced. Moreover, to initialize the assonance investigation, a two-parameter study will be conducted.

In Chapter 3, a simulation-based study will be made to examine assonant mechanisms related to resonator frequency, combination, sequencing, and host material stiffness variation. The definition of the test cases for resonator-type, resonator sequence, and material variation will be addressed.

The application of energy harvesting for a locally resonant acoustic metamaterial is presented in Chapter 4. The derivation of the electromotive force (EMF) and the magnetic field will be shown. An experimental scheme will be introduced to validate the derived EMF and magnetic field equations. Furthermore, the investigation of energy harvesting scenarios will be carried out for the best assonant cases. Finally, the conclusions are reported, and recommendations for the future are made in Chapter 5. 


\section{CHAPTER II}

\section{THEORETICAL MODELING FOR LOCALLY RESONANT ACOUSTIC METAMATERIAL}

\section{$\underline{2.1 \text { Introduction }}$}

As shown in Figure 1.12, a discrete element model representation of the AMB is considered. Each unit-cell in the barrier is represented using a host mass $\left(m_{1}\right)$ and external stiffness $\left(k_{1}\right)$ along with a local mass $\left(m_{2}\right)$ and stiffness $\left(k_{2}\right)$ that represents the attached resonator. The base parametric setting was defined for the $\mathrm{AMB}$, as shown below in Table 2.1. These base parameters will be utilized for both the discrete model analysis and simulation model, which will be discussed in this chapter.

Table 2.1: Summary of base parameters for LRAM.

\begin{tabular}{c|c|c|c|c}
\hline Description & Symbol & Base Value & Unit & $\begin{array}{c}\text { Fixed/ } \\
\text { Variable }\end{array}$ \\
\hline The total length of the AM barrier & $L_{T}$ & 2 & $\mathrm{~m}$ & Fixed \\
\hline Lattice length & $L$ & 0.1 & $\mathrm{~m}$ & Fixed \\
\hline Number of unit-cells & $N$ & 20 & - & Fixed \\
\hline Host material's mass per unit-cell & $m_{1}$ & 1 & $\mathrm{~kg}$ & Fixed \\
\hline Inclusion mass per unit-cell & $m_{2}$ & 1 & $\mathrm{~kg}$ & Fixed \\
\hline Total static mass & $T_{m s}$ & 40 & $\mathrm{~kg}$ & Fixed \\
\hline Host material's stiffness per unit-cell & $k_{1}$ & 200000000 & $\mathrm{~N} / \mathrm{m}$ & Variable \\
\hline Inclusion stiffness per unit-cell & $k_{2}$ & 9869604 & $\mathrm{~N} / \mathrm{m}$ & Variable \\
\hline Total static stiffness & $k_{s t}$ & 10526316 & $\mathrm{~N} / \mathrm{m}$ & Fixed \\
\hline Base value of resonator frequency & $f_{0}$ & 500 & $\mathrm{~Hz}$ & Variable \\
\hline Optimal damping & $c_{d}$ & 10 & $\mathrm{~N} . \mathrm{s} / \mathrm{m}$ & Fixed \\
\hline
\end{tabular}




\subsection{Discrete Model Analysis}

Consider a one-dimensional infinite mass-in-mass lattice system, as shown in Figure 2.1 that represents a locally resonant acoustic metamaterial (LRAM). This depiction will be subject to the same base parameters defined in Table 2.1; however, the parameter for the total lattice length is assumed to be infinite in this modeling, just so that it will be easier to look at the dispersion relations without having to consider the reflections and transmission from boundaries.

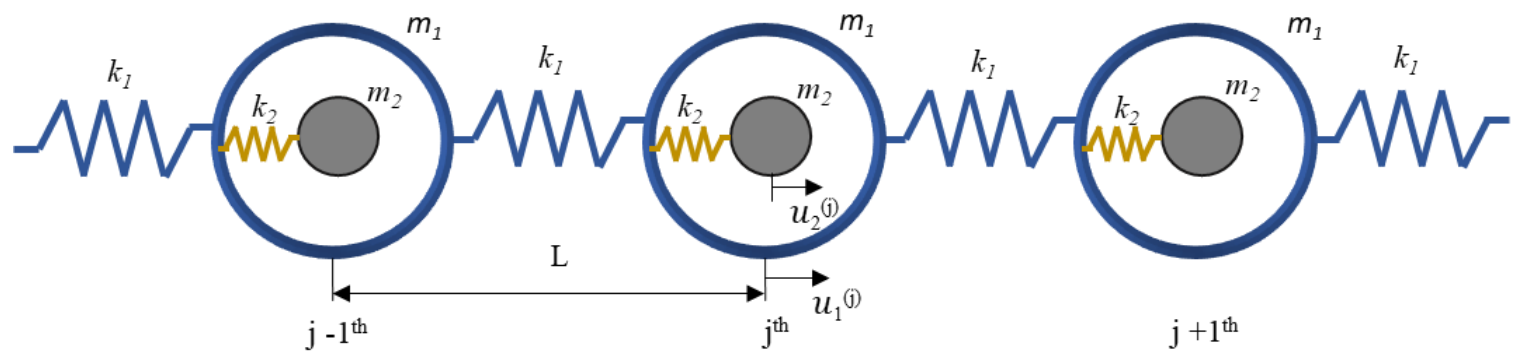

Figure 2.1: Discrete mass-spring lattice model for a locally resonant acoustic metamaterial.

Now, writing the equation of motion for the $\mathrm{j}^{\text {th }}$ mass:

$$
\begin{gathered}
m_{1}^{(j)} \ddot{u}_{1}^{(j)}+k_{1}\left(2 u_{1}^{(j)}-u_{1}^{(j-1)}-u_{1}^{(j+1)}\right)+k_{2}\left(u_{1}^{(j)}-u_{2}^{(j)}\right)=0 \\
m_{2}^{(j)} \ddot{u}_{2}^{(j)}+k_{2}\left(u_{2}^{(j)}-u_{1}^{(j)}\right)=0
\end{gathered}
$$

Equation (2.1) and (2.2) represent a second-order linear differential equation, therefore by enforcing the superposition theorem, we can assume a harmonic solution for $\mathrm{u}_{1}$ and $\mathrm{u}_{2}$ as stated in Equation (2.3) and (2.4) below.

$$
\begin{gathered}
u_{1}^{j}=A_{1} e^{i(\gamma x+\gamma L-\omega t)} \\
u_{2}^{j}=A_{2} e^{i(-\omega t)}
\end{gathered}
$$


Where $\mathrm{A}$ is a complex wave displacement amplitude, $\gamma$ is wave number, $\omega$ is circular frequency. The substitution of Equation (2.3) and (2.4) into (2.1) and (2.2) leads to the normalized dispersion relation between wavenumber and circular frequency, as stated below in Equation (2.5).

$$
\gamma L=\cos ^{-1}\left(1-\frac{\omega^{2}\left(m_{s t}-m_{1} \Omega^{2}\right)}{2 k_{1}\left(1-\Omega^{2}\right)}\right)
$$

The normalized excitation frequency is defined in Equation (2.5) as, $\Omega=\frac{\omega}{\omega_{0}}, \omega_{0}=\sqrt{\frac{k_{2}}{m_{2}}}$ represents the natural frequency of the resonators, the static mass for a unit-cell is defined as, $m_{s_{t}}$ $=m_{1}+m_{2}$, whereas, $\gamma L$ is normalized wave number. Looking at the limiting cases for Equation (2.5) when $\Omega=1$ leads to the denominator to be zero, thus showing that the wavenumber becomes purely imaginary, in this case, there will be no propagation.

Figure 2.2 illustrates a dispersion relation between the normalized wave number and excitation frequency. The shaded area in the chart shows the bandgap frequency range; in this zone, the wavenumber becomes purely imaginary. Note that for the base setting the local resonance frequency, $\omega_{0}$, for all units are chosen to be $500 \mathrm{~Hz}$. For the $m_{\text {ratio }}\left(\right.$ i.e. $m_{2} / m_{1}=1$ ) selected for this case, the bandgap range extends from about $(\Omega=1$ to 1.414). Bandgaps are a result of multiple Bragg scattering and destructive interference that takes place in the structure due to periodic changes in the impedance. 


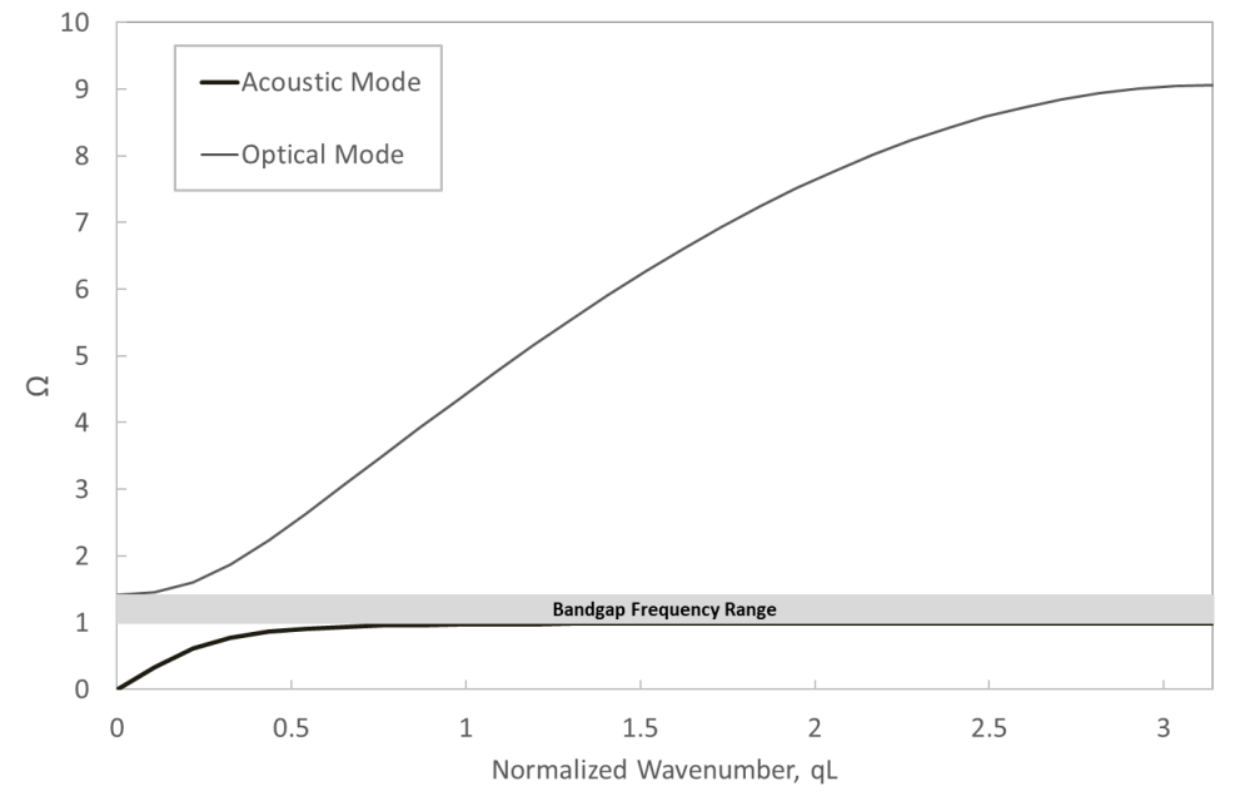

Figure 2.2: Dispersion curves and bandgap frequency range for an LRAM with a local resonance frequency of $500 \mathrm{~Hz}$.

Figure 2.3 depicts the model of the LRAM as an effective mass, $m_{e f f}$ which is represented by a single mass which would produce the same motion as $m_{1}$. Furthermore, the existence of the local mass $m_{2}$ would be ignored while its effect will be absorbed within the $m_{\text {eff }}$.

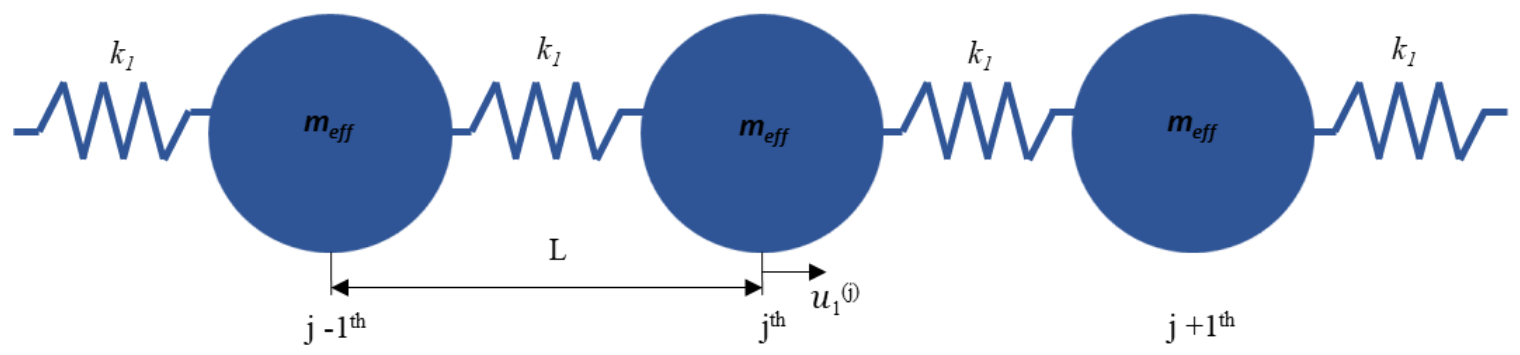

Figure 2.3: Effective mass model of a discrete mass-spring lattice model for a locally resonant acoustic metamaterial.

Since the dynamic response of $m_{1}$ and $m_{e f f}$ are identical for a prescribed input excitation, it is possible to enforce this condition to achieve an equation for the effective mass as: 


$$
m_{e f f}=m_{s t}+\frac{m_{2} \Omega^{2}}{\left(1-\Omega^{2}\right)}
$$

Observing the limiting cases for Equation (2.6), it is possible to see that within the range $1<\Omega<\sqrt{1+m_{\text {ratio }}}$ the effective mass will become negative, and attenuation will occur within that limit. The figure below shows the relation between the $m_{e f f} / m_{1}$ and $\Omega$.

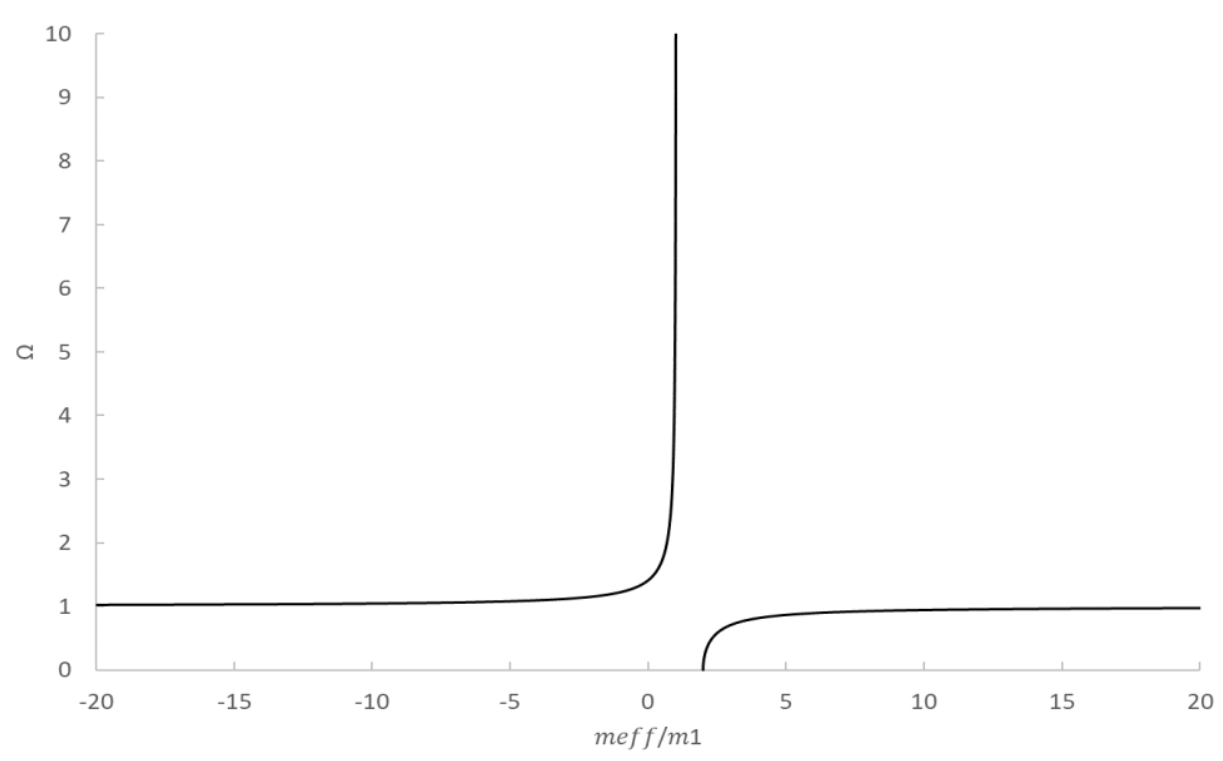

Figure 2.4: Normalized effective mass vs. normalized effective excitation frequency.

\subsection{Simulation Model}

A one-dimensional discrete element model of the AMB with defined base parameters given in Table 2.1 is developed on Abaqus for numerical simulation study, as shown in Figure 2.5. A free end boundary condition was assumed at the transmission end of the AMB, along with the input excitation being applied at the first unit on the incident side. These boundary conditions were kept consistent throughout all case studies. 


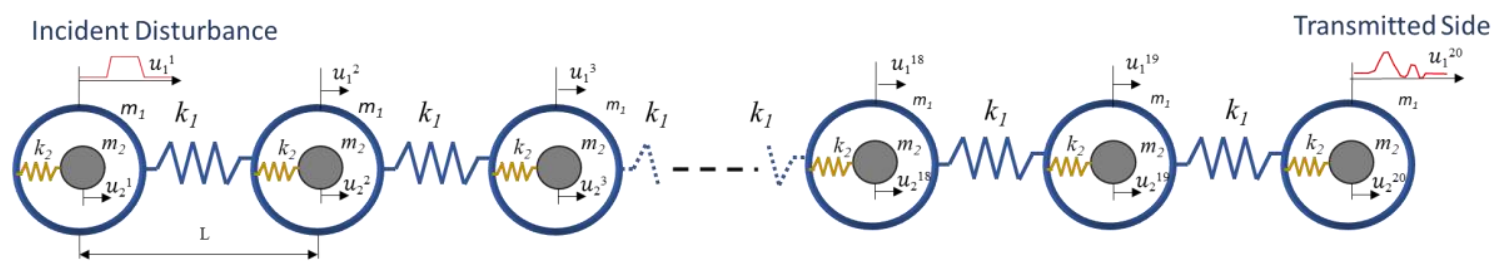

Figure 2.5: Simulation model for a one-dimensional finite length $A M B$ with $N(=20)$ units.

For all cases to be studied except the check case study, a step displacement input pulse shown in Figure $2.5 \mathrm{a}$ is used as the incident disturbance. It has a total duration of $(0.5 \mathrm{~ms})$ with a rise and fall time of $(0.1 \mathrm{~ms})$ and a peak displacement amplitude of $0.01 \mathrm{~m}$. Its frequency spectrum is shown in Figure 2.5b. The dominant frequency content for this input pulse extends up to 2500 Hz.

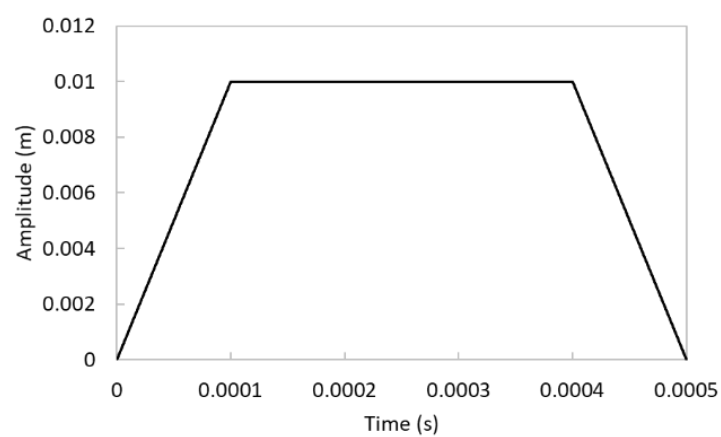

(a)

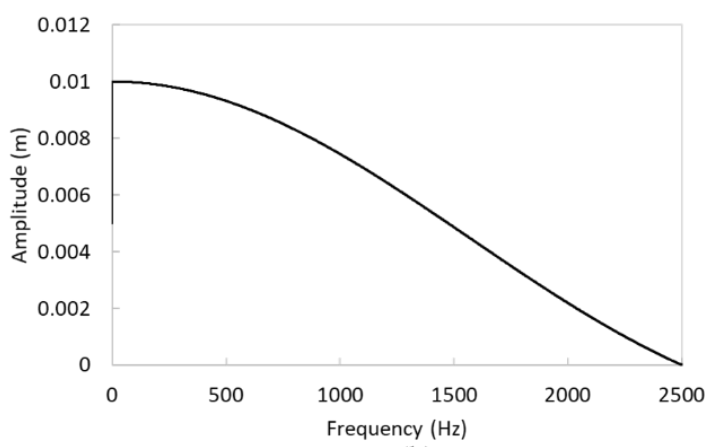

(b)

Figure 2.6: (a) Displacement time history and (b) frequency spectrum for the step input excitation.

\subsubsection{Check Case Study}

The check case study defined below in Table 2.2 shows a list of single frequency harmonic excitation ( $\mathrm{C} 1$ to $\mathrm{C} 5)$, which are used to interrogate the simulation model for the LRAM in order to verify the predicted dispersion behavior as stated above on the discrete model analysis. Furthermore, studies (C6 to C9) are used to estimate an optimal amount of structural damping that can represent actual losses in a system which are unavoidable in practical structures. Moreover, 
these studies will further aid for the optimization of computational efficiency in order to advance convergence to steady-state with only a negligible impact on the output amplitude compared to that of the undamped case.

Table 2.2: Summary of check $(C)$ cases.

\begin{tabular}{|c|c|c|c|c|c|}
\hline $\begin{array}{l}\text { Case } \\
\text { No. }\end{array}$ & Description & $\begin{array}{l}\text { Input } \\
\text { Exc. } \\
\text { Freq. }\end{array}$ & $\begin{array}{l}\text { Resonator } \\
\text { Config. }\end{array}$ & $\begin{array}{l}\text { Damping } \\
\text { (N.s/m) }\end{array}$ & Remarks \\
\hline $\mathrm{C} 1$ & $\begin{array}{l}\text { AMB - Propagation } \\
\text { check }\end{array}$ & $200 \mathrm{~Hz}$ & $20 \times 500 \mathrm{~Hz}$ & 10 & $\begin{array}{l}\text { Propagation - acoustic } \\
\text { mode }\end{array}$ \\
\hline $\mathrm{C} 2$ & $\begin{array}{l}\text { AMB - Attenuation } \\
\text { check }\end{array}$ & $525 \mathrm{~Hz}$ & $20 \times 500 \mathrm{~Hz}$ & 10 & $\begin{array}{l}\text { Close to lower limit in the } \\
\text { bandgap }\end{array}$ \\
\hline $\mathrm{C} 3$ & $\begin{array}{l}\text { AMB - Attenuation } \\
\text { check }\end{array}$ & $690 \mathrm{~Hz}$ & $20 \times 500 \mathrm{~Hz}$ & 10 & $\begin{array}{l}\text { Close to the upper limit in } \\
\text { the bandgap }\end{array}$ \\
\hline $\mathrm{C} 4$ & $\begin{array}{l}\text { AMB - Propagation } \\
\text { check }\end{array}$ & $1000 \mathrm{~Hz}$ & $20 \times 500 \mathrm{~Hz}$ & 10 & Propagation - optical mode \\
\hline $\mathrm{C} 5$ & $\begin{array}{l}\text { AMB - Attenuation } \\
\text { check }\end{array}$ & $505 \mathrm{~Hz}$ & $20 \times 500 \mathrm{~Hz}$ & 10 & Just within bandgap \\
\hline C6 & AMB - Damping check & $200 \mathrm{~Hz}$ & $20 \times 500 \mathrm{~Hz}$ & 0 & No damping \\
\hline $\mathrm{C} 7$ & AMB - Damping check & $200 \mathrm{~Hz}$ & $20 \times 500 \mathrm{~Hz}$ & 1 & Intermediate damping \\
\hline $\mathrm{C} 8$ & AMB - Damping check & $200 \mathrm{~Hz}$ & $20 \times 500 \mathrm{~Hz}$ & 10 & Intermediate damping \\
\hline C9 & AMB - Damping check & $200 \mathrm{~Hz}$ & 20x500 Hz & 100 & Highest damping \\
\hline
\end{tabular}

\subsubsection{Baseline Case Study}

The baseline case study consists of an elastic waveguide without local resonators but having the same static mass and stiffness. Therefore, it has a mass of $m_{s t}$ and stiffness of $k_{1}$ per unit-cell and overall stiffness of $k_{s t}=19 / k_{1}$ across the AMB. The performance of the baseline case helps set a reference for the performance of AMB cases against a non-metamaterial barrier. 
Table 2.3: Summary of overall baseline case for comparison to all AMB cases.

\begin{tabular}{c|c|c|c|c|c}
\hline $\begin{array}{c}\text { Case } \\
\text { No. }\end{array}$ & Description & $\begin{array}{c}\text { Input } \\
\text { Exc. }\end{array}$ & $\begin{array}{c}\text { Resonator } \\
\text { Config. }\end{array}$ & $\begin{array}{c}\text { Damping } \\
\text { (N.s/m) }\end{array}$ & Remarks \\
\hline Baseline & $\begin{array}{c}\text { Elastic media } \\
\text { without inclusions }\end{array}$ & $0.5 \mathrm{~ms}$ Step & None & 10 & $\begin{array}{c}\text { Has the same static mass } \\
\text { and stiffness as all the } \\
\text { AMB cases }\end{array}$ \\
\hline
\end{tabular}

The minimization of three metrics is considered to assess the relative performance of targeted spectral transformations across the AMBs with prescribed variations in design. These are (i) the displacement transmissibility $\left(\mathrm{D}^{*}\right)$, (ii) the power spectral transmissibility $\left(\mathrm{S}^{*}\right)$, and (iii) normalized wave speed $\left(\mathrm{C}^{*}\right)$. Their definitions are as follows:

$$
D^{*}=\frac{\left|u_{1}^{(N)}\right|_{\text {cases }}}{\left|u_{1}^{(N)}\right|_{\text {baseline }}}
$$

where $\left|u_{1}^{(N)}\right|$ is the absolute max amplitude of the output displacement recorded at the $N^{\text {th }}\left(20^{\text {th }}\right)$ unit of the AMB.

$$
S^{*}=\frac{\left\{A_{1}^{(N)}\right\}_{\text {cases }}}{\left\{A_{1}^{(N)}\right\}_{\text {baseline }}} \times 1000
$$

where $A_{1}^{(N)}$ is the area under the power spectrum up to $2500 \mathrm{~Hz}$ for the output displacement recorded at the $N^{\text {th }}\left(20^{\text {th }}\right)$ unit of the AMB. Note that the scale factor of 1000 is used for presentation purposes of results.

$$
C^{*}=\frac{\left.c\right|_{\text {cases }}}{\left.c\right|_{\text {baseline }}}
$$

where $c$ is wavespeed recorded from simulations. 
It is desirable to minimize the displacement amplitude as well as the power spectral area of the transmitted wave in order to enhance the isolation of the transmitted-side media from the incident disturbance via the AMB. Further, as will be discussed later on, minimizing the wave speed through the AMB is found to correlate well with enhanced resonator action. However, $\mathrm{C}^{*}$ is considered more as a means to understand the assonant mechanisms involved rather than a metric per say.

\subsubsection{Two-Parameter Study}

The optimization of an AMB to mitigate incoming broadband pulse involves several dependent and independent parameters. A preliminary study involving two key parameters was first undertaken to minimize the design space required to investigate assonant mechanisms. In order to conduct this investigation, we impose the constrain that alternating external stiffnesses can be distinct namely $k_{\text {even }}$ and $k_{\text {odd }}$. As seen in Figure $2.6 k_{\text {even }}$ and $k_{\text {odd }}$ are alternated within the AMB. For the second parameter all resonator frequency for any particular case is the same. In addition, the static mass and stiffness remains unaltered.

The variation of the external stiffness is to study the effect of Bragg scattering and impedance mismatch between the unit cells. Figure 2.7 represents an AMB with nineteen external stiffnesses that are connecting twenty-unit cells with an alternating stiffness arrangement.

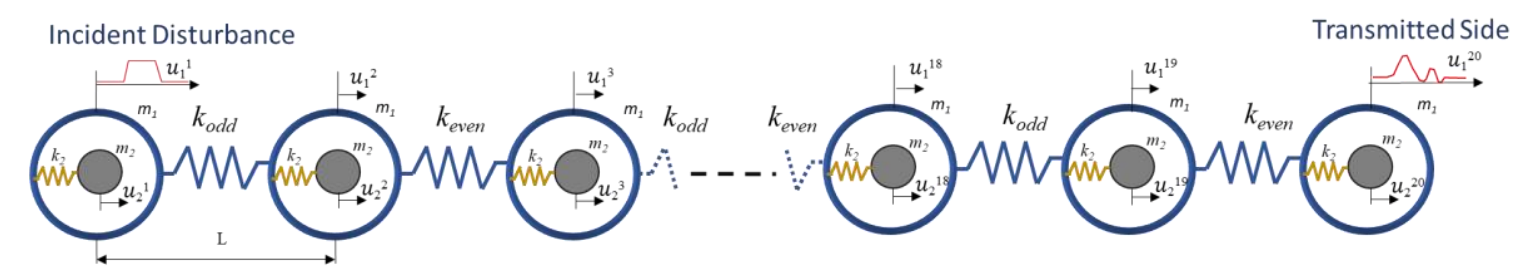

Figure 2.7: Discrete element model for two-parameter study.

Periodic elements, when subject to a particular disturbance, tend to oscillate in opposite phases. Consequently, the wave moves neither to the right nor to the left. This scenario is equivalent 
to Bragg reflection of x-rays in optics: when Bragg condition is satisfied, a traveling wave cannot propagate in a lattice. Still, through successive reflections back and forth, a standing wave is a setup. Moreover, with the introduction of alternate variations of relatively hard and soft external stiffness, as seen in Figure 2.7, it is also possible to create an impedance mismatch within the AMB. Note that static stiffness, $k_{s t}$ is kept fixed for all cases. The alternating stiffness variation is achieved by:

$$
\begin{gathered}
\frac{1}{k_{\text {st }}}=\frac{10}{k_{\text {odd }}}+\frac{9}{k_{\text {even }}}, \\
\nwarrow=\frac{k_{\text {odd }}}{k_{\text {even }}}, \\
\tilde{\omega}=\frac{\omega_{0}}{2500 \mathrm{~Hz}},
\end{gathered}
$$

Where $\xi_{\zeta}$ is stiffness ratio, and $\tilde{\omega}$ is normalized frequency. With the given two Equations (2.10) and (2.11), one can obtain value for $k_{\text {even }}$ and $k_{\text {odd }}$.

The second parameter is the variation of resonator frequencies that lie within the dominant bandwidth of the input spectrum. In practice, if a design restriction of having $N$ units within the total available length $\left(L_{T}\right)$ is imposed, one could have up to $N$ distinct local resonance frequencies for these units. However, it is useful to consider further design restrictions, where only smaller number of specific sets of resonator types are possible. The numerical simulation model developed in the previous section will be used to investigate some of the assonant mechanisms such as those related to resonator frequency combination, sequencing, and host material stiffness. Figure 2.8 shows the case matrix for the two-parameter study (SK cases) to be conducted. The resonator variation with 25 different resonator frequencies is shown extending across the column, and 50 cases of stiffness variation are listed row-wise. A single case study takes approximately 3-4 minutes to complete a simulation; therefore, a Python code was used to automate the simulation in Abaqus. 
Furthermore, a post-processing code is used to extract and populate the results; MATLAB was used in this case. The codes can be found in Appendix A.1 and A.2.

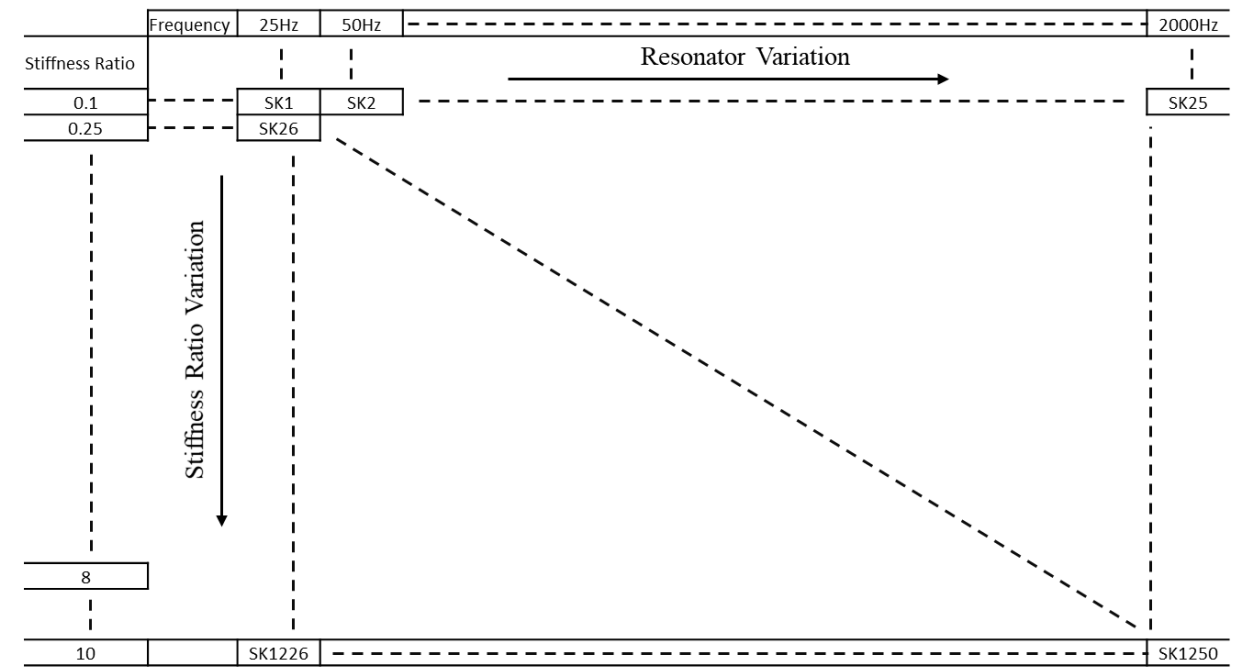

Figure 2.8: Case matrix diagram for the two-parameter study.

It is expected that the results and observations from this study can provide preliminary guidelines for a more optimal design of resonant metamaterials as well as serve as a starting point for formal assonant investigations.

\subsection{Results and Discussion}

For each simulation, the output displacement time history at the last or $N\left(=20^{\text {th }}\right)$ unit's host mass is extracted, and the metrics as per Equation (2.7 - 2.9) are reported for the baseline and twoparameter study. Further, the frequency spectrum is examined to understand relative contributions from specific bandwidths within the dominant frequency range of interest in the input. Observations and trends are also discussed.

\subsubsection{Check Case Study}

The output displacement time histories for a set of check cases are shown in Figure 2.8. These correspond to the cases with harmonic frequency excitations having an input amplitude of $0.01 \mathrm{~m}$ spanning the frequency range of interest to probe the bandgap behavior of the AMB. 


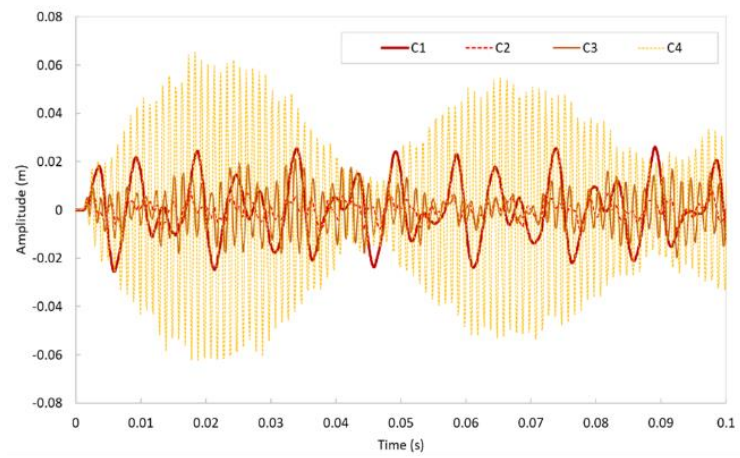

(a)

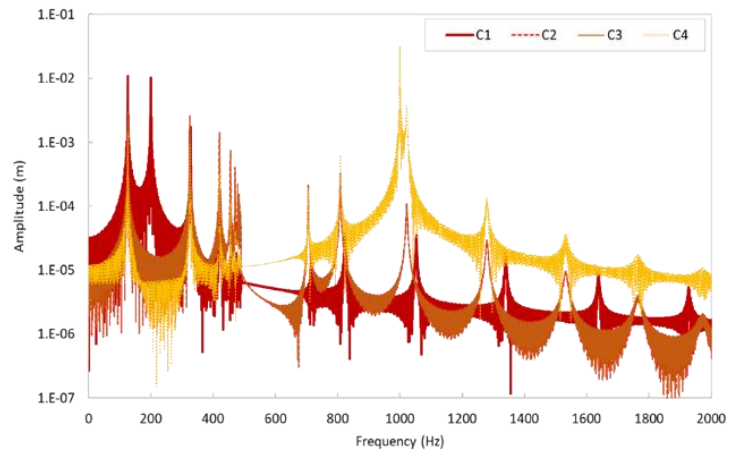

(b)

Figure 2.9: Output displacement (a) time histories and (b) frequency spectra for check cases.

It is seen that for cases $\mathrm{C} 1$ (acoustic mode) and C4 (optical mode), propagation behavior is observed. In contrast, attenuation commensurate with frequency location within the bandgap is obtained for cases C2 (close to the lower limit of bandgap) and C3 (close to the upper limit of bandgap) indicating correlation with expected behavior for such locally resonant AM. Inspecting the frequency spectrum for these cases in Figure 2.9 (b) shows a reduction in amplitude within the bandgap frequency range that extends from about 500 to $707 \mathrm{~Hz}$ for excitation frequencies within the bandgap frequency range. This verifies the simulation model for the AMB.

Additionally, variation in damping was considered to estimate an optimal amount of damping, ensuring relatively faster convergence to steady state without any appreciable reduction to the output amplitude for the undamped case. While these results are not shown here, a damping value of $10 \mathrm{~N} . \mathrm{s} / \mathrm{m}$ was found to provide an optimal influence.

\subsubsection{Baseline Case Study}

The verified simulation model having the base parametric setting was used as the basis for the simulation models to investigate the two-parameter and assonant studies. This baseline case consists of an elastic waveguide having the same static mass and stiffness as the metamaterial cases. As seen in Figure 2.10, the peak output displacement amplitude for this case is more than twice the peak input amplitude of $(0.01 \mathrm{~m})$, which is expected for a free end boundary condition the 
displacement response at the last unit-cell is doubled. This serves as a baseline measure of performance for all the AMB cases. It was also found that the dominant frequency range of the output frequency spectrum remains unaltered. Following the baseline case, the resonator frequency variation cases are considered.

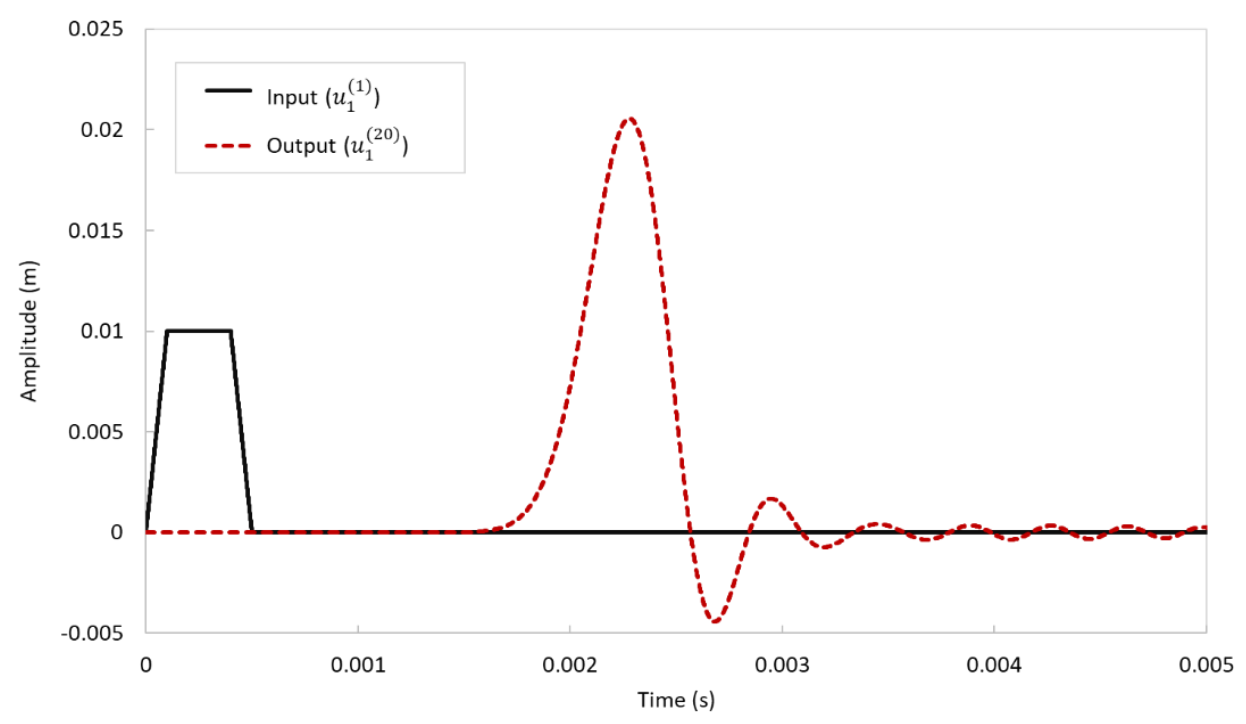

Figure 2.10: Input and output displacement time histories for the baseline (SR7) case.

\subsubsection{Two Parameter Study}

The influence of the alternating arrangement of host material's stiffness in parallel with resonator variation was investigated. The purpose of this study is to aid with the selection of best resonator frequencies and stiffness ratios, which will later be used in the investigation of assonant cases. Figure 2.11 shows the surface plot result of each case $\mathrm{D}^{*}$ plotted against normalized external stiffness ratio and normalized frequency.

In general, low-frequency resonators within the bandwidth of interest are not preferable. A slight trend of an overall improvement in $\mathrm{D}^{*}$ performance can be noticed as resonator frequencies are increased; however, having local resonant frequencies near the max bandwidth region is not recommended. 


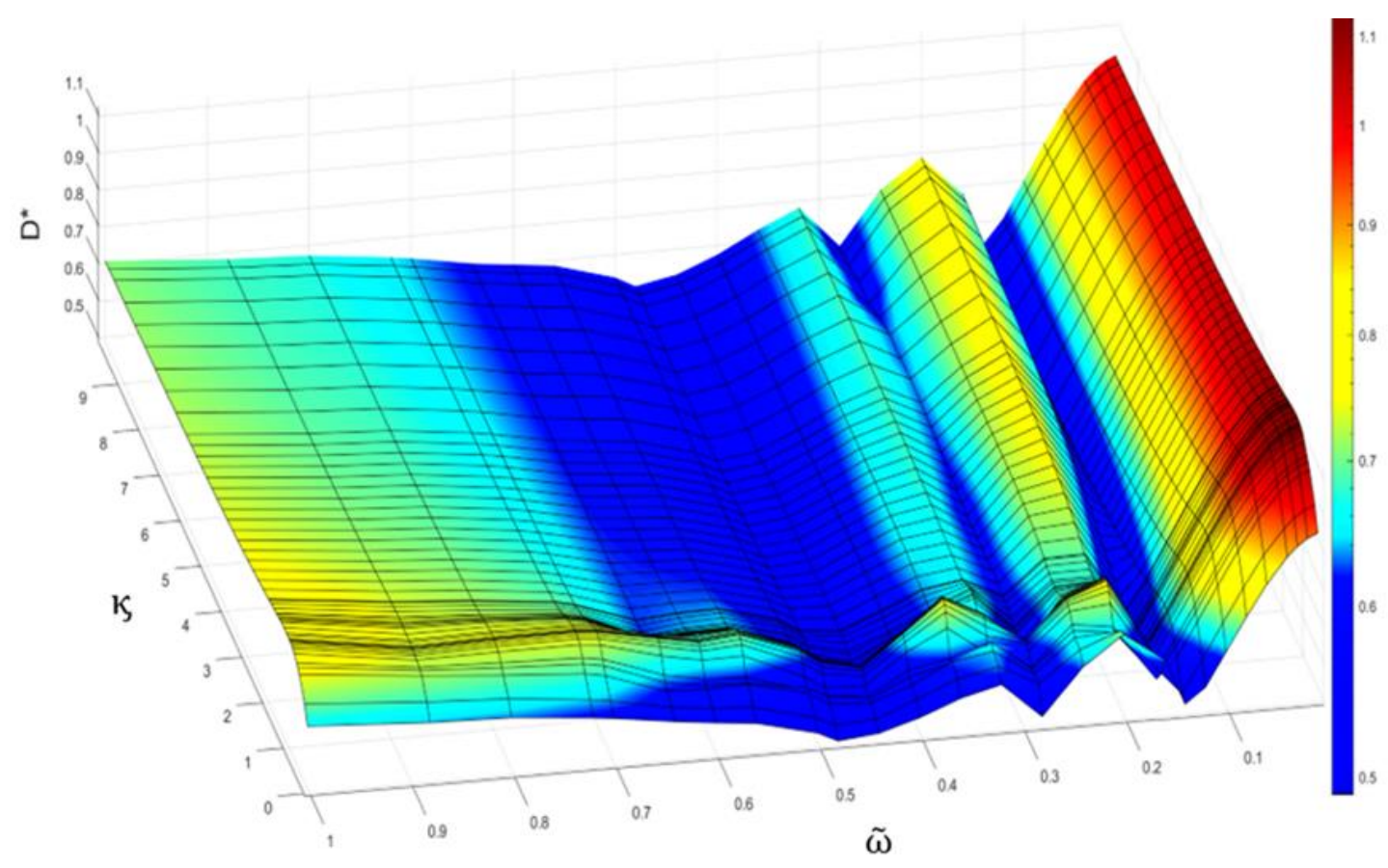

Figure 2.12: Surface plot for two-parameter study.

It is found desirable to choose resonator frequencies in the region where $\tilde{\omega}=0.12-0.16$, $0.24-0.32$, and $0.4-0.6$ ) of the max bandwidth frequency. These subsequent regions are approximately doubled or halved from each other; thus, they can be associated with an octave spacing. Octave refers to the interval between one frequency and its double or half.

The alternating arrangement of external stiffness introduces an impedance mismatch. As a result, reflections and transmission of waves are created between host masses connected by an alternating stiffness arrangement; thus, the overall wave propagation can be reduced. From this study, it is found that when the ratio between $k_{\text {odd }}$ and $k_{\text {even }}$ approaches to unity attenuation performance of the AMB decreases.

In agreement with the analogy inspired by the literary device assonance, it can be noted that the transmissibility performance of the $\mathrm{AMB}$ is influenced more so by the variation of local resonators (vowels), rather than by the alternating arrangement of external stiffness (consonants). 


\subsection{Summary}

The main goal of this chapter is to study the attenuation performance of an AMB with two varying parameters, alternating external stiffness arrangement and resonator frequency. Theoretical model with an infinite mass-in-mass lattice is used to interrogate the dispersion relation of AM for the defined based parameters. Moreover, a check case study was used to verify the simulation model by looking at propagating and attenuating cases. Also, optimal damping investigation was made and found a reasonable value that gave the best computational efficiency without having a significant loss in amplitude.

A two-parameter study was developed to investigate the effects of alternating external stiffness ratio and resonator frequency. Based on the result of the study the following conclusion were made:

- It is found that very low resonator frequencies $\left(<1 / 10^{\text {th }}\right.$ of max bandwidth $)$ are not preferable.

- Subsequent regions with enhanced $\mathrm{D}^{*}$ metrics are found to be spaced in an octave like manner.

- Simultaneously, it is found that when the ratio of the alternating stiffnesses approaches unity the attenuation performance of the AMB is reduced.

- In agreement with the analogy inspired by the literary device assonance, it can be noted that the transmissibility performance of the AMB is predominately influenced by the variation of local resonators (vowels), rather than the alternating arrangement of external stiffness (consonants). 


\section{CHAPTER III}

\section{ASSONANCE-BASED DESIGN CASE STUDY}

\subsection{Introduction}

Following the two-parameter study made in the previous section, this chapter will study some of the assonant mechanisms such as those related to resonator frequency combination, sequencing, and host material stiffness. The numerical simulation model developed in chapter 2 will be used to demonstrate the ability of the AMB to mitigate the propagation of a broadband step input pulse from an incident-side elastic media to a transmitted-side elastic media. The purpose of this investigation is to serve as an exploratory study to gauge the influence of assonant mechanisms' on the metamaterial's performance using a few specific metrics for targeted spectral transformations across the AMB.

\subsection{Numerical Parametric Study}

In this study, some of the assonant mechanisms such as those related to resonator frequency combination, sequencing, and host material stiffness are considered and investigated using simulation for a metamaterial barrier used to mitigate the propagation of a broadband step input pulse from an incident-side elastic media to a transmitted-side elastic media.

\subsubsection{Definition of Test Cases}

Several categories of test cases are defined to initialize the exploration of assonant mechanisms for resonant frequency combinations, sequencing of resonators, and host material's stiffness. 


\subsubsection{Resonator-Type Variation Case (SR)}

The first assonant mechanism studied is the resonator frequency combination (or SR cases) for the AMB. Note that, the static mass and stiffness is kept fixed for all cases in this study as a means of ensuring a consistency in terms of overall weight and compliance. The only parameter varied in this case study is the local resonance frequency and that is altered by changing the resonator stiffness, $k_{2}$. The case study in this section is divided into 4 different subcategories, single, two, four as well as $\mathrm{N}(=20)$ distinct resonator-type cases.

\subsection{Single Resonator-Type Case}

The single resonator-type cases are summarized in Table 3.1. These cases consider various single resonance frequencies within the dominant frequency bandwidth $(<2500 \mathrm{~Hz})$ of the input step pulse. By using the results from the two-parameter study the resonator frequencies chosen for the single resonator-type cases are summarized in Table 3.1.

Table 3.1: Summary of single resonator-type (SR) cases.

\begin{tabular}{c|c|c|c|c|c}
\hline $\begin{array}{c}\text { Case } \\
\text { No. }\end{array}$ & Description & $\begin{array}{c}\text { Input } \\
\text { Exc. }\end{array}$ & $\begin{array}{c}\text { Resonator } \\
\text { Config. }\end{array}$ & $\begin{array}{c}\text { Damping } \\
(\mathbf{N . s} / \mathbf{m})\end{array}$ & Remarks \\
\hline SR1 & AMB - Single resonator-type & $\begin{array}{c}0.5 \mathrm{~ms} \\
\text { Step }\end{array}$ & $\begin{array}{c}20 \times 500 \\
\mathrm{~Hz}\end{array}$ & 10 & Mid res. freq. within inp. b/w \\
\hline SR2 & AMB - Single resonator-type & $\begin{array}{c}0.5 \mathrm{~ms} \\
\text { Step }\end{array}$ & $\begin{array}{c}20 x 1000 \\
\mathrm{~Hz}\end{array}$ & 10 & Mid res. freq. within inp. b/w \\
\hline SR10 & AMB - Single resonator-type & $\begin{array}{c}0.5 \mathrm{~ms} \\
\text { Step }\end{array}$ & $\begin{array}{c}20 \mathrm{x} 100 \\
\mathrm{~Hz}\end{array}$ & 10 & Low res. freq. within inp. b/w \\
\hline SR11 & AMB - Single resonator-type & $\begin{array}{c}0.5 \mathrm{~ms} \\
\text { Step }\end{array}$ & $\begin{array}{c}20 \mathrm{x} 250 \\
\mathrm{~Hz}\end{array}$ & 10 & Low res. freq. within inp. b/w \\
\hline SR12 & AMB - Single resonator-type & $\begin{array}{c}0.5 \mathrm{~ms} \\
\text { Step }\end{array}$ & $\begin{array}{c}20 x 2000 \\
\mathrm{~Hz}\end{array}$ & 10 & High res. freq. within inp. b/w \\
\hline
\end{tabular}




\subsection{Two Resonator-Type Case}

For the two resonator-type cases, combinations of two mid and high resonator frequencies within the input bandwidth are considered. These cases are summarized in Table 3.2.

Table 3.2: Summary of two resonator-type (SR) cases.

\begin{tabular}{c|c|c|c|c|c}
\hline $\begin{array}{c}\text { Case } \\
\text { No. }\end{array}$ & Description & $\begin{array}{c}\text { Input } \\
\text { Exc. }\end{array}$ & Resonator Config. & $\begin{array}{c}\text { Damping } \\
\text { (N.s/m) }\end{array}$ & Remarks \\
\hline SR3 & $\begin{array}{c}\text { AMB - Two } \\
\text { resonator-type }\end{array}$ & $\begin{array}{c}0.5 \mathrm{~ms} \\
\text { Step }\end{array}$ & $10 \times 500+10 \times 1000 \mathrm{~Hz}$ & 10 & $\begin{array}{c}\text { Mid res. freq. within inp. } \\
\text { b/w }\end{array}$ \\
\hline SR4 & $\begin{array}{c}\text { AMB - Two } \\
\text { resonator-type }\end{array}$ & $\begin{array}{c}0.5 \mathrm{~ms} \\
\text { Step }\end{array}$ & $10 \times 250+10 \times 500 \mathrm{~Hz}$ & 10 & $\begin{array}{c}\text { Low res. freq. within } \\
\text { inp. b/w }\end{array}$ \\
\hline
\end{tabular}

\subsection{Four Resonator-Type Case}

Most cases considered for resonator frequency variation are four resonator-type cases. Several potential resonator frequencies spacing and the number of resonators in each set are regarded as summarized in Table 3.3. Notable among these are the evenly spaced, spectral amplitude-proportional, octave spacing, and coincident bandgap case. The observation made from the two-parameter study suggests that there is a slight correlation which shows the doubling of subsequent frequency bandwidth that give the best D* performance. Similarly, an octave spacing (doubling of the spacing frequency band between successive resonator frequencies) is introduced for SR13 and SR14. 
Table 3.3: Summary of four resonator-type (SR) cases.

\begin{tabular}{|c|c|c|c|c|c|}
\hline Case No. & Description & $\begin{array}{l}\text { Input } \\
\text { Exc. }\end{array}$ & Resonator Config. & $\begin{array}{l}\text { Damping } \\
\text { (N.s/m) }\end{array}$ & Remarks \\
\hline SR5 & $\begin{array}{l}\text { AMB - Four } \\
\text { resonator-type }\end{array}$ & $\begin{array}{l}0.5 \mathrm{~ms} \\
\text { Step }\end{array}$ & $\begin{array}{c}5 \times 250+5 \times 500+ \\
5 \times 750+5 \times 1000 \mathrm{~Hz}\end{array}$ & 10 & $\begin{array}{c}\text { Evenly spaced and distributed } \\
\text { resonators }\end{array}$ \\
\hline SR8 & $\begin{array}{l}\text { AMB - Four } \\
\text { resonator-type }\end{array}$ & $\begin{array}{l}0.5 \mathrm{~ms} \\
\text { Step }\end{array}$ & $\begin{array}{c}9 \times 250+6 \times 500+ \\
3 \times 750+2 \times 1000 \mathrm{~Hz}\end{array}$ & 10 & $\begin{array}{l}\text { Higher number of low } \\
\text { frequency resonators }\end{array}$ \\
\hline SR9 & $\begin{array}{l}\text { AMB - Four } \\
\text { resonator-type }\end{array}$ & $\begin{array}{l}0.5 \mathrm{~ms} \\
\text { Step }\end{array}$ & $\begin{array}{c}6 \times 250+5 \times 500+ \\
5 \times 750+4 \times 1000 \mathrm{~Hz}\end{array}$ & 10 & $\begin{array}{l}\text { Spectral amplitude- } \\
\text { proportional number of } \\
\text { resonators }\end{array}$ \\
\hline SR13 & $\begin{array}{l}\text { AMB - Four } \\
\text { resonator-type }\end{array}$ & $\begin{array}{l}0.5 \mathrm{~ms} \\
\text { Step }\end{array}$ & $\begin{array}{c}5 \times 100+5 \times 300+ \\
5 \times 700+5 \times 1500 \mathrm{~Hz}\end{array}$ & 10 & $\begin{array}{l}\text { Octave spacing of resonator } \\
\text { frequencies }\end{array}$ \\
\hline SR14 & $\begin{array}{l}\text { AMB - Four } \\
\text { resonator-type }\end{array}$ & $\begin{array}{l}0.5 \mathrm{~ms} \\
\text { Step }\end{array}$ & $\begin{array}{c}6 \times 100+6 \times 300 \\
+5 \times 700+3 \times 1500 \mathrm{~Hz}\end{array}$ & 10 & $\begin{array}{l}\text { Spectral amplitude- } \\
\text { proportional number with } \\
\text { octave frequency spacing for } \\
\text { resonators }\end{array}$ \\
\hline SR15 & $\begin{array}{l}\mathrm{AMB}-\text { Four } \\
\text { resonator-type }\end{array}$ & $\begin{array}{l}0.5 \mathrm{~ms} \\
\text { Step }\end{array}$ & $\begin{array}{c}4 \times 250+5 \times 500 \\
+5 \times 750+6 \times 1000 \mathrm{~Hz}\end{array}$ & 10 & $\begin{array}{l}\text { Reverse order of number of } \\
\text { resonators as that of SR9 }\end{array}$ \\
\hline SR16 & $\begin{array}{l}\text { AMB - Four } \\
\text { resonator-type }\end{array}$ & $\begin{array}{l}0.5 \mathrm{~ms} \\
\text { Step }\end{array}$ & $\begin{array}{c}5 \times 250+5 \times 353.55+ \\
5 \times 500+5 \times 707 \mathrm{~Hz}\end{array}$ & 10 & $\begin{array}{l}\text { Resonator frequencies } \\
\text { coincident with bandgap limits }\end{array}$ \\
\hline SR17 & $\begin{array}{l}\mathrm{AMB}-\text { Four } \\
\text { resonator-type }\end{array}$ & $\begin{array}{l}0.5 \mathrm{~ms} \\
\text { Step }\end{array}$ & $\begin{array}{c}5 \times 500+5 \times 707+ \\
5 \times 1000+5 \times 1414 \mathrm{~Hz}\end{array}$ & 10 & $\begin{array}{l}\text { Resonator frequencies } \\
\text { coincident with bandgap limits }\end{array}$ \\
\hline SR21 & $\begin{array}{l}\text { AMB - Four } \\
\text { resonator-type }\end{array}$ & $\begin{array}{l}0.5 \mathrm{~ms} \\
\text { Step }\end{array}$ & $\begin{aligned} 5 \times 50 & +5 \times 150+5 \times 350 \\
& +5 \times 750 \mathrm{~Hz}\end{aligned}$ & 10 & $\begin{array}{l}\text { Octave spacing with lower } \\
\text { starting frequency }\end{array}$ \\
\hline SR22 & $\begin{array}{l}\text { AMB - Four } \\
\text { resonator-type }\end{array}$ & $\begin{array}{l}0.5 \mathrm{~ms} \\
\text { Step }\end{array}$ & $\begin{array}{c}7 \times 150+7 \times 450+ \\
5 \times 1050+1 \times 2250 \mathrm{~Hz}\end{array}$ & 10 & $\begin{array}{l}\text { Octave spacing with higher } \\
\text { starting frequency }\end{array}$ \\
\hline
\end{tabular}

\subsection{N Distinct Resonator-Type Case}

The final set of cases considered for resonator frequency variation are the $N$ distinct resonator-type cases summarized in Table 3.4. This test case showcases the applications of 20 different resonators within the AMB. 
Table 3.4: Summary of $N(=20)$ distinct resonator-type $(S R)$ cases.

\begin{tabular}{c|c|c|c|c|c}
\hline $\begin{array}{c}\text { Case } \\
\text { No. }\end{array}$ & Description & $\begin{array}{c}\text { Input } \\
\text { Exc. }\end{array}$ & Resonator Config. & $\begin{array}{c}\text { Damping } \\
\text { (N.s/m) }\end{array}$ & Remarks \\
\hline SR6 & $\begin{array}{c}\text { AMB }-\mathrm{N}(=20) \text { distinct } \\
\text { resonator-type }\end{array}$ & $\begin{array}{c}0.5 \mathrm{~ms} \\
\text { Step }\end{array}$ & $\begin{array}{c}100 \mathrm{~Hz} \text { spacing from } \\
100 \text { to } 2000 \mathrm{~Hz}\end{array}$ & 10 & $\begin{array}{c}\text { Wider spacing over wider } \\
\text { frequency bandwidth }\end{array}$ \\
\hline SR18 & $\begin{array}{c}\text { AMB }-\mathrm{N}(=20) \text { distinct } \\
\text { resonator-type }\end{array}$ & $\begin{array}{c}0.5 \mathrm{~ms} \\
\text { Step }\end{array}$ & $\begin{array}{c}50 \mathrm{~Hz} \text { spacing from } 100 \\
\text { to } 1000 \mathrm{~Hz}\end{array}$ & 10 & $\begin{array}{c}\text { Closer spacing in lower } \\
\text { frequency bandwidth }\end{array}$ \\
\hline SR19 & $\begin{array}{c}\text { AMB - N (=20) distinct } \\
\text { resonator-type }\end{array}$ & $\begin{array}{c}0.5 \mathrm{~ms} \\
\text { Step }\end{array}$ & $\begin{array}{c}50 \mathrm{~Hz} \text { spacing from } \\
1000 \text { to } 2000 \mathrm{~Hz}\end{array}$ & 10 & $\begin{array}{c}\text { Closer spacing in higher } \\
\text { frequency bandwidth }\end{array}$ \\
\hline SR20 & $\begin{array}{c}\text { AMB - N (=20) distinct } \\
\text { resonator-type }\end{array}$ & $\begin{array}{c}0.5 \mathrm{~ms} \\
\text { Step }\end{array}$ & $\begin{array}{c}50 \mathrm{~Hz} \text { spacing from } 500 \\
\text { to } 1500 \mathrm{~Hz}\end{array}$ & 10 & $\begin{array}{c}\text { Closer spacing in mid } \\
\text { frequency bandwidth }\end{array}$ \\
\hline
\end{tabular}

\subsubsection{Resonator Sequence Variation Case (SQ)}

The second assonant mechanism explored is the sequencing (or SQ cases) for a given set of resonators within the AMB from incident-side to the transmitted-side.

Table 3.5: Summary of resonator sequence variation (SQ) cases.

\begin{tabular}{c|c|c|c|c|c}
\hline $\begin{array}{c}\text { Case } \\
\text { No. }\end{array}$ & Description & $\begin{array}{c}\text { Input } \\
\text { Exc. }\end{array}$ & Resonator Config. & $\begin{array}{c}\text { Damping } \\
\text { (N.s/m) }\end{array}$ & Remarks \\
\hline SQ1 & $\begin{array}{c}\text { AMB - Resonator } \\
\text { sequence variation }\end{array}$ & $\begin{array}{c}0.5 \mathrm{~ms} \\
\text { Step }\end{array}$ & $\begin{array}{c}6 \times 100+6 \times 300+5 \times 700+ \\
3 \times 1500 \mathrm{~Hz}\end{array}$ & 10 & $\begin{array}{c}\text { Same as SR14 - Best case for } \\
\text { resonator frequency variation }\end{array}$ \\
\hline SQ2 & $\begin{array}{c}\text { AMB - Resonator } \\
\text { sequence variation }\end{array}$ & $\begin{array}{c}0.5 \mathrm{~ms} \\
\text { Step }\end{array}$ & $\begin{array}{c}3 \times 1500+5 \times 700+6 \times 300+ \\
6 \times 100 \mathrm{~Hz}\end{array}$ & 10 & $\begin{array}{c}\text { Mirror sequence of SQ1 } \\
\text { (SR14) }\end{array}$ \\
\hline SQ3 & $\begin{array}{c}\text { AMB - Resonator } \\
\text { sequence variation }\end{array}$ & $\begin{array}{c}0.5 \mathrm{~ms} \\
\text { Step }\end{array}$ & $\begin{array}{c}(1 \times 1500) \times 3+(1 \times 100+1 \times 300 \\
+1 \times 700) \times 2+(1 \times 100+ \\
1 \times 300) \mathrm{Hz}\end{array}$ & 10 & $\begin{array}{c}\text { Fully interspersed sequencing } \\
\text { of SQ1 (SR14) }\end{array}$ \\
\hline SQ4 & $\begin{array}{c}\text { AMB - Resonator } \\
\text { sequence variation }\end{array}$ & $\begin{array}{c}0.5 \mathrm{~ms} \\
\text { Step }\end{array}$ & $\begin{array}{c}6 \times 100+5 \times 700+6 \times 300+ \\
3 \times 1500 \mathrm{~Hz}\end{array}$ & 10 & $\begin{array}{c}\text { Block sequences switched for } \\
\text { SQ1 (SR14) retaining lowest } \\
\text { frequency (100 Hz) block first }\end{array}$ \\
\hline SQ5 & $\begin{array}{c}\text { AMB - Resonator } \\
\text { sequence variation }\end{array}$ & $\begin{array}{c}0.5 \mathrm{~ms} \\
\text { Step }\end{array}$ & $\begin{array}{c}6 \times 100+5 \times 700+6 \times 300+ \\
3 \times 1500 \mathrm{~Hz}\end{array}$ & 10 & $\begin{array}{c}\text { Block sequences switched for } \\
\text { SQ1 (SR14) retaining lowest } \\
\text { frequency (100 Hz) block first }\end{array}$ \\
\hline SQ6 & $\begin{array}{c}\text { AMB - Resonator } \\
\text { sequence variation }\end{array}$ & $\begin{array}{c}0.5 \mathrm{~ms} \\
\text { Step }\end{array}$ & $\begin{array}{c}6 \times 100+5 \times 700+3 \times 1500+ \\
6 \times 300 \mathrm{~Hz}\end{array}$ & 10 & $\begin{array}{c}\text { Block sequences switched for } \\
\text { SQ1 (SR14) retaining lowest } \\
\text { frequency (100 Hz) block first }\end{array}$ \\
\hline
\end{tabular}




\begin{tabular}{c|c|c|c|c|c}
\hline SQ7 & $\begin{array}{c}\text { AMB - Resonator } \\
\text { sequence variation }\end{array}$ & $\begin{array}{c}0.5 \mathrm{~ms} \\
\text { Step }\end{array}$ & $\begin{array}{c}6 \times 100+3 \times 1500+6 \times 300+ \\
5 \times 700 \mathrm{~Hz}\end{array}$ & 10 & $\begin{array}{c}\text { Block sequences switched for } \\
\text { SQ1 (SR14) retaining lowest } \\
\text { frequency (100 Hz) block first }\end{array}$ \\
\hline SQ8 & $\begin{array}{c}\text { AMB - Resonator } \\
\text { sequence variation }\end{array}$ & $\begin{array}{c}0.5 \mathrm{~ms} \\
\text { Step }\end{array}$ & $\begin{array}{c}6 \times 100+3 \times 1500+5 \times 700+ \\
6 \times 300 \mathrm{~Hz}\end{array}$ & 10 & $\begin{array}{c}\text { Block sequences switched for } \\
\text { SQ1 (SR14) retaining lowest } \\
\text { frequency (100 Hz) block first }\end{array}$ \\
\hline SQ9 & $\begin{array}{c}\text { AMB - Resonator } \\
\text { sequence variation }\end{array}$ & $\begin{array}{c}0.5 \mathrm{~ms} \\
\text { Step }\end{array}$ & $\begin{array}{c}1 \times 100) \times 3+(1 \times 700+1 \times 300 \\
+1 \times 100) \times 2+(1 \times 300+ \\
1 \times 100) \mathrm{Hz}\end{array}$ & 10 & $\begin{array}{c}\text { Fully interspersed sequencing } \\
\text { of SQ1 (SR14) but in reverse } \\
\text { order }\end{array}$ \\
\hline SQ10 & $\begin{array}{c}\text { AMB - Resonator } \\
\text { sequence variation }\end{array}$ & $\begin{array}{c}0.5 \mathrm{~ms} \\
\text { Step }\end{array}$ & $\begin{array}{c}6 \times 300+5 \times 700+3 \times 1500+ \\
6 \times 100 \mathrm{~Hz}\end{array}$ & 10 & $\begin{array}{c}\text { Block sequences switched for } \\
\text { SQ1 (SR14) }\end{array}$ \\
\hline SQ11 & $\begin{array}{c}\text { AMB - Resonator } \\
\text { sequence variation }\end{array}$ & $\begin{array}{c}0.5 \mathrm{~ms} \\
\text { Step }\end{array}$ & $\begin{array}{c}5 \times 700+6 \times 100+6 \times 300+ \\
3 \times 1500 \mathrm{~Hz}\end{array}$ & 10 & $\begin{array}{c}\text { Block sequences switched for } \\
\text { SQ1 (SR14) }\end{array}$ \\
\hline SQ12 & $\begin{array}{c}\text { AMB - Resonator } \\
\text { sequence variation }\end{array}$ & $\begin{array}{c}0.5 \mathrm{~ms} \\
\text { Step }\end{array}$ & $\begin{array}{c}3 \times 1500+6 \times 100+6 \times 300+ \\
5 \times 700 \mathrm{~Hz}\end{array}$ & 10 & $\begin{array}{c}\text { Block sequences switched for } \\
\text { SQ1 (SR14) }\end{array}$ \\
\hline
\end{tabular}

This variation in sequencing is considered for the best performing resonator frequency variation (SR) case as detailed in Table 3.5. Sequencing variations such as mirroring, fully interspersed, and block switching among others are considered.

\subsubsection{Material Variation Case (SM)}

Finally, variation in host material stiffness (SM cases) are considered. The purposes of these cases are to explore the performance change (as evidenced by the metrics) that can be obtained by varying the stiffness of the host material of the AMB. Combinations of relatively hard and soft host material stiffnesses in comparison to the base value of the host material stiffness are considered along with their sequencing. 
Table 3.6: Summary of host material stiffness variation (SM) cases.

\begin{tabular}{|c|c|c|c|c|c|c|}
\hline $\begin{array}{l}\text { Case } \\
\text { No. }\end{array}$ & Description & $\begin{array}{l}\text { Input } \\
\text { Exc. }\end{array}$ & Resonator Config. & $\begin{array}{c}\text { Damping } \\
(\mathrm{N} / \mathrm{m})\end{array}$ & $\begin{array}{l}\text { Host Matl. } \\
\text { Stiffness } \\
\text { Config. }\end{array}$ & Remarks \\
\hline SM1 & $\begin{array}{c}\text { AMB }- \text { Host } \\
\text { material stiffness } \\
\text { variation }\end{array}$ & $\begin{array}{l}0.5 \mathrm{~ms} \\
\text { Step }\end{array}$ & $\begin{array}{c}6 \times 100+5 \times 700+ \\
3 \times 1500+6 \times 300 \mathrm{~Hz} \\
(\text { Same as SQ6 - } \\
\text { Baseline) }\end{array}$ & 10 & $\begin{array}{l}\text { Same as SQ6 - } \\
\text { Baseline }\end{array}$ & $\begin{array}{c}\text { Same as SQ6 - Best } \\
\text { case for sequence } \\
\text { variation }\end{array}$ \\
\hline SM2 & $\begin{array}{c}\text { AMB }- \text { Host } \\
\text { material stiffness } \\
\text { variation }\end{array}$ & $\begin{array}{c}0.5 \mathrm{~ms} \\
\text { Step }\end{array}$ & $\begin{array}{c}6 \times 100+5 \times 700+ \\
3 \times 1500+6 \times 300 \mathrm{~Hz} \\
(\text { Same as SQ6 - } \\
\text { Baseline) }\end{array}$ & 10 & $(\mathrm{H}-\mathrm{S}-) \times 9+\mathrm{H}$ & $\begin{array}{l}\text { Alternating 'Hard' }(\mathrm{H}) \\
\text { and 'Soft' }(\mathrm{S}) \text { host } \\
\text { material stiffness } \\
\text { sequence }\end{array}$ \\
\hline SM3 & $\begin{array}{c}\text { AMB - Host } \\
\text { material stiffness } \\
\text { variation }\end{array}$ & $\begin{array}{l}0.5 \mathrm{~ms} \\
\text { Step }\end{array}$ & $\begin{array}{c}6 \times 100+5 \times 700+ \\
3 \times 1500+6 \times 300 \mathrm{~Hz} \\
(\text { Same as SQ6 - } \\
\text { Baseline) }\end{array}$ & 10 & $(\mathrm{~S}-\mathrm{H}-) \times 9+\mathrm{S}$ & $\begin{array}{c}\text { Alternating 'Soft' (S) } \\
\text { and 'Hard' }(\mathrm{H}) \text { host } \\
\text { material stiffness } \\
\text { sequence }\end{array}$ \\
\hline SM4 & $\begin{array}{c}\mathrm{AMB}-\text { Host } \\
\text { material stiffness } \\
\text { variation }\end{array}$ & $\begin{array}{c}0.5 \mathrm{~ms} \\
\text { Step }\end{array}$ & $\begin{array}{c}6 \times 100+5 \times 700+ \\
3 \times 1500+6 \times 300 \mathrm{~Hz} \\
(\text { Same as SQ6 - } \\
\text { Baseline) }\end{array}$ & 10 & $\begin{array}{c}(\mathrm{H}-) \times 10+(\mathrm{S}- \\
) \times 9\end{array}$ & $\begin{array}{l}\text { 'Hard' (H) host matl } \\
\text { stiffness for first } 10 \\
\text { units and 'Soft' (S) } \\
\text { host matl stiffness for } \\
\text { last } 9 \text { units }\end{array}$ \\
\hline SM5 & $\begin{array}{c}\mathrm{AMB}-\text { Host } \\
\text { material stiffness } \\
\text { variation }\end{array}$ & $\begin{array}{c}0.5 \mathrm{~ms} \\
\text { Step }\end{array}$ & $\begin{array}{c}6 \times 100+5 \times 700+ \\
3 \times 1500+6 \times 300 \mathrm{~Hz} \\
(\text { Same as SQ6 - } \\
\text { Baseline) }\end{array}$ & 10 & $\begin{array}{c}(\mathrm{S}-) \times 10+(\mathrm{H}- \\
) \times 9\end{array}$ & $\begin{array}{l}\text { 'Soft' (S) host matl } \\
\text { stiff for first } 10 \text { units } \\
\text { and 'Hard' (H) host } \\
\text { matl stiff for last } 9 \\
\text { units }\end{array}$ \\
\hline SM6 & $\begin{array}{c}\mathrm{AMB}-\text { Host } \\
\text { material stiffness } \\
\text { variation }\end{array}$ & $\begin{array}{c}0.5 \mathrm{~ms} \\
\text { Step }\end{array}$ & $\begin{array}{c}6 \times 100+5 \times 700+ \\
3 \times 1500+6 \times 300 \mathrm{~Hz} \\
(\text { Same as SQ6 - } \\
\text { Baseline) }\end{array}$ & 10 & $\begin{array}{c}(\mathrm{H}-) \times 9+\mathrm{S}+(\mathrm{H}- \\
) \times 9\end{array}$ & $\begin{array}{l}1 \text { 'Soft' (S) host matl } \\
\text { stiffness between } 9 \\
\text { 'Hard' (H) host matl } \\
\text { stiffness on either side }\end{array}$ \\
\hline SM7 & $\begin{array}{c}\mathrm{AMB}-\text { Host } \\
\text { material stiffness } \\
\text { variation }\end{array}$ & $\begin{array}{l}0.5 \mathrm{~ms} \\
\text { Step }\end{array}$ & $\begin{array}{c}6 \times 100+5 \times 700+ \\
3 \times 1500+6 \times 300 \mathrm{~Hz} \\
(\text { Same as SQ6 - } \\
\text { Baseline) }\end{array}$ & 10 & $\begin{array}{c}(\mathrm{H}-) \times 6+(\mathrm{S}-) \times 5 \\
+(\mathrm{H}-) \times 3+(\mathrm{S}- \\
) \times 6\end{array}$ & $\begin{array}{c}\text { Block-wise host } \\
\text { material stiffness } \\
\text { variation for SQ6 } \\
\text { (Baseline) case }\end{array}$ \\
\hline SM8 & $\begin{array}{c}\mathrm{AMB}-\text { Host } \\
\text { material stiffness } \\
\text { variation }\end{array}$ & $\begin{array}{c}0.5 \mathrm{~ms} \\
\text { Step }\end{array}$ & $\begin{array}{c}6 \times 100+5 \times 700+ \\
3 \times 1500+6 \times 300 \mathrm{~Hz} \\
(\text { Same as SQ6 - } \\
\text { Baseline) }\end{array}$ & 10 & $\begin{array}{c}(\mathrm{S}-) \mathrm{x} 6+(\mathrm{H}-) \times 5 \\
+ \\
(\mathrm{S}-) \times 3+(\mathrm{H}-) \times 6\end{array}$ & $\begin{array}{c}\text { Block-wise host } \\
\text { material stiffness } \\
\text { variation for SQ6 } \\
\text { (Baseline) case }\end{array}$ \\
\hline SM9 & $\begin{array}{c}\mathrm{AMB}-\text { Host } \\
\text { material stiffness } \\
\text { variation }\end{array}$ & $\begin{array}{l}0.5 \mathrm{~ms} \\
\text { Step }\end{array}$ & $\begin{array}{c}6 \times 100+5 \times 700+ \\
3 \times 1500+6 \times 300 \mathrm{~Hz} \\
(\text { Same as SQ6 - } \\
\text { Baseline) }\end{array}$ & 10 & $\begin{array}{c}(\mathrm{H}-) \mathrm{x} 6+(\mathrm{S}-) \mathrm{x} 5 \\
+ \\
(\mathrm{S}-) \mathrm{x} 3+(\mathrm{H}-) \mathrm{x} 6\end{array}$ & $\begin{array}{l}\text { Block-wise host } \\
\text { material stiffness } \\
\text { variation for SQ6 } \\
\text { (Baseline) case }\end{array}$ \\
\hline
\end{tabular}

It must be noted that for all these cases the static mass and especially the static (overall) stiffness of the waveguide is not changed from that of the baseline elastic waveguide. Moreover, 
specific material, geometric or mechanical design consideration involved in realizing these stiffnesses in practice are not explored in this study. However, these cases are expected to provide guidance on the general trend in terms of host material selection of individual units within the AMB in order to enhance its overall performance.

\subsubsection{Discussion of Results}

For each simulation, the output displacement time history at the last or Nth (=20th) unit's host mass is extracted and the metrics as per Eq. 1-3 are reported for the assonant cases. Further, the its frequency spectrum is examined to understand relative contributions from specific bandwidths within the dominant frequency range of interest in the input. Observations and trends are also discussed.

\subsubsection{Resonator-Type Variation Case}

The results for single, two, four as well as $\mathrm{N}(=20)$ distinct resonator-type cases will be discussed.

\subsection{Single Resonator-Type Case}

The output displacement histories and spectra for single resonator-type cases are shown in Figure 3.1 and Figure 3.2 respectively. It can be noted from the transmissibility plot shown in Figure 3.3 that low resonator frequencies $-100 \mathrm{~Hz}(\mathrm{SR} 10)$ or $250 \mathrm{~Hz}$ (SR11) yields poor attenuation performance compared to that of the mid-frequency (SR1 and SR2) or high resonator frequency cases (SR12). 


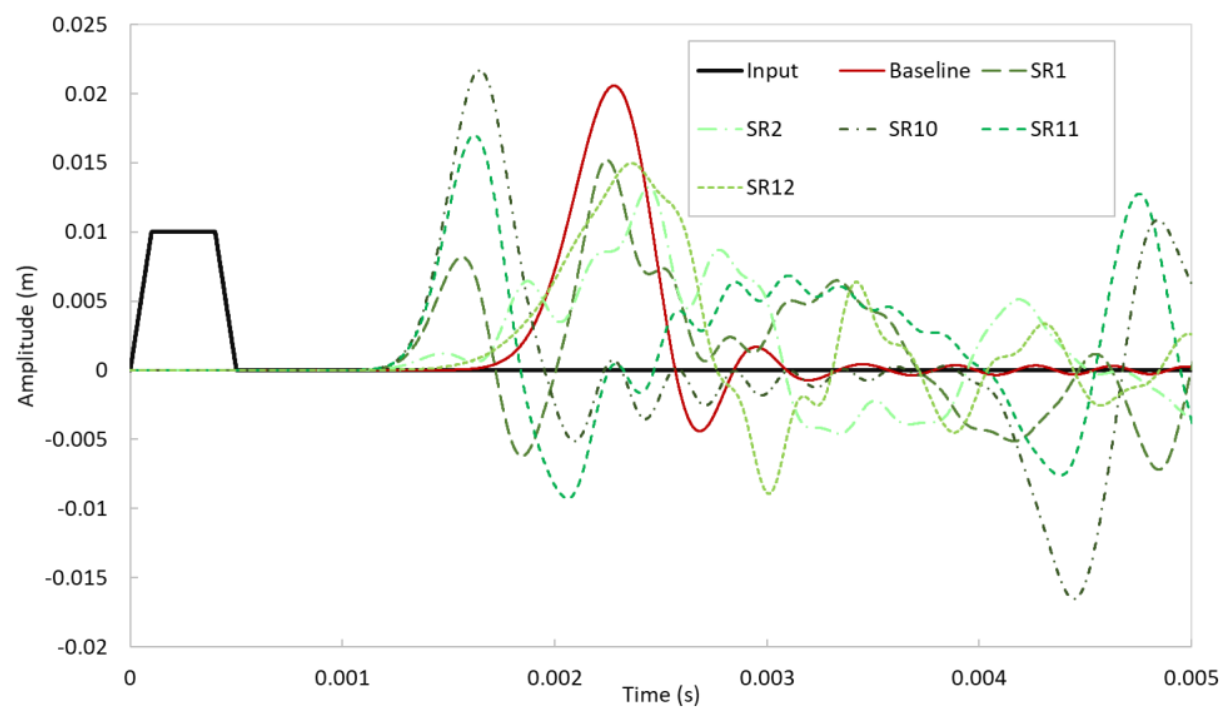

Figure 3.1: Input, baseline (SR7) and output displacement time histories for the single resonator-

type cases.

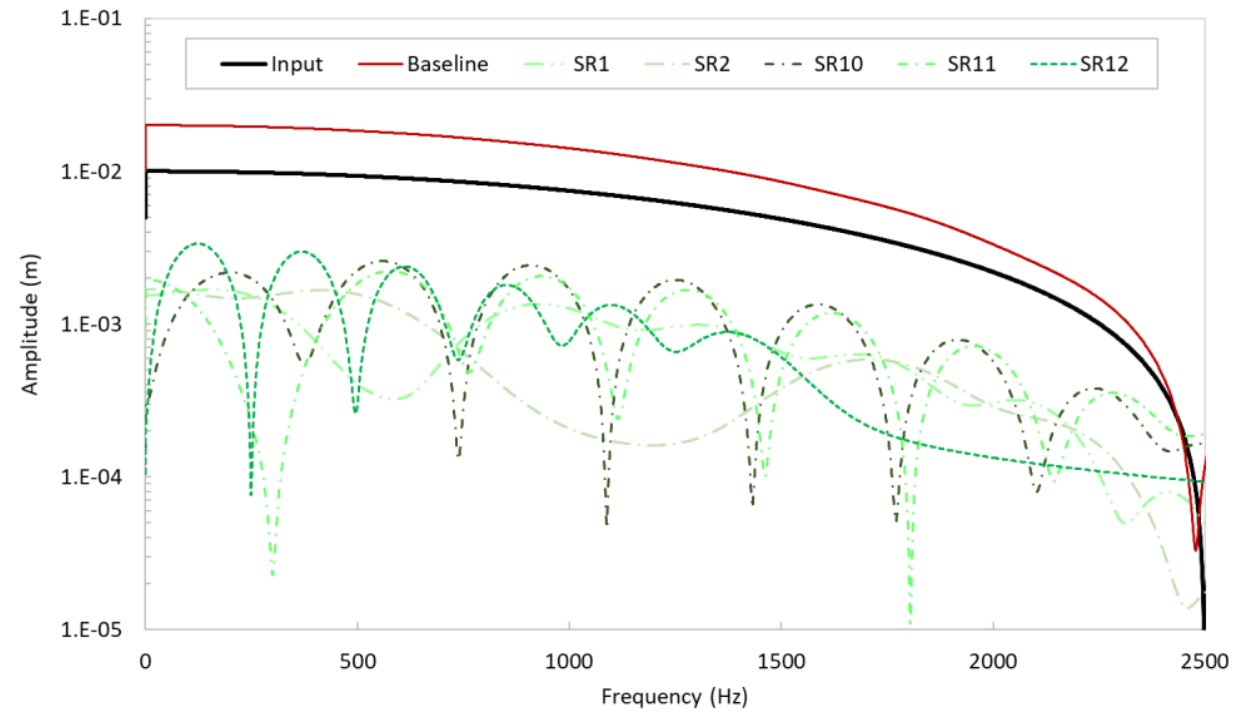

Figure 3.2: Input, baseline (SR7) and output displacement frequency spectra for the single resonator-type cases. 


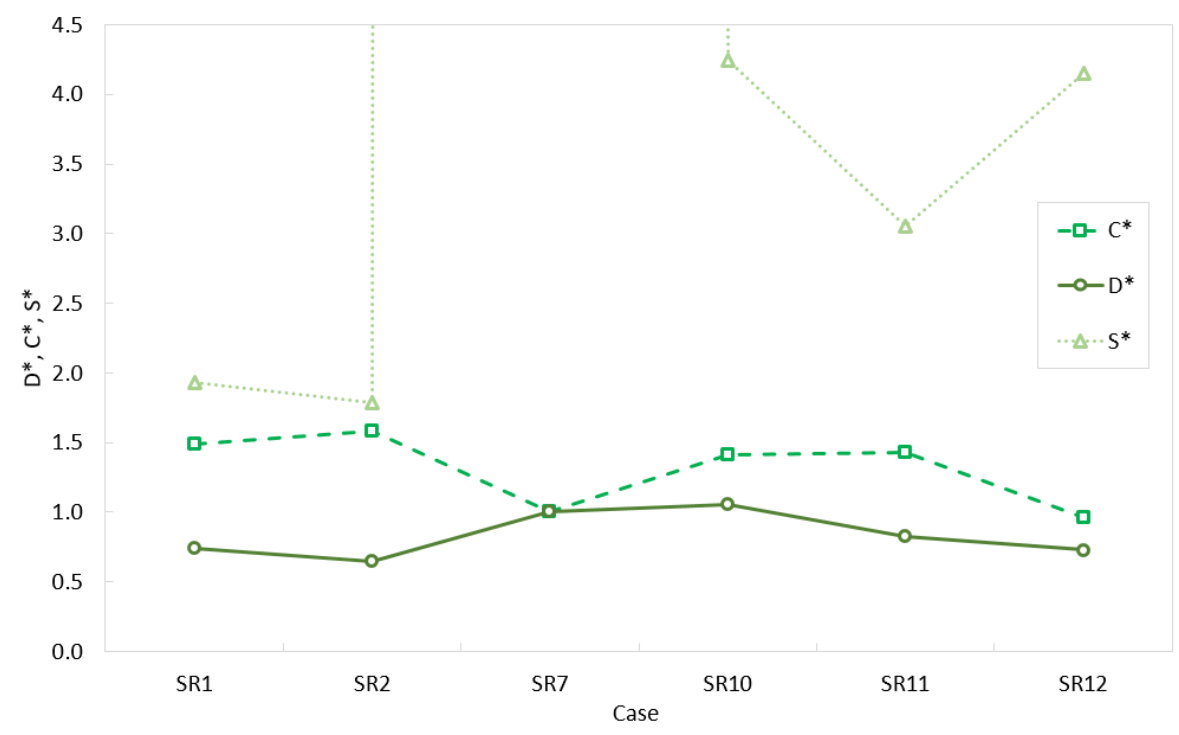

Figure 3.3: Output displacement $\left(D^{*}\right)$ and power spectral $\left(S^{*}\right)$ transmissibility and normalized wave speed $\left(C^{*}\right)$ for the single resonator-type cases

\subsection{Two Resonator-Type Case}

The results for the two resonator-cases are summarized in Figures 3.4, 3.5, and 3.6. Again, having two resonator combination with mid-range frequencies (as in SR3) is found to be slightly better than low-range frequencies (SR4) for $\mathrm{D}^{*}$, and both are found to perform much better than the baseline. This case study, in conjunction with the two-parameter study, indicates, in general, that having only low resonator frequencies within the bandwidth of interest is less preferable in order to enhance performance as per the metrics considered. 


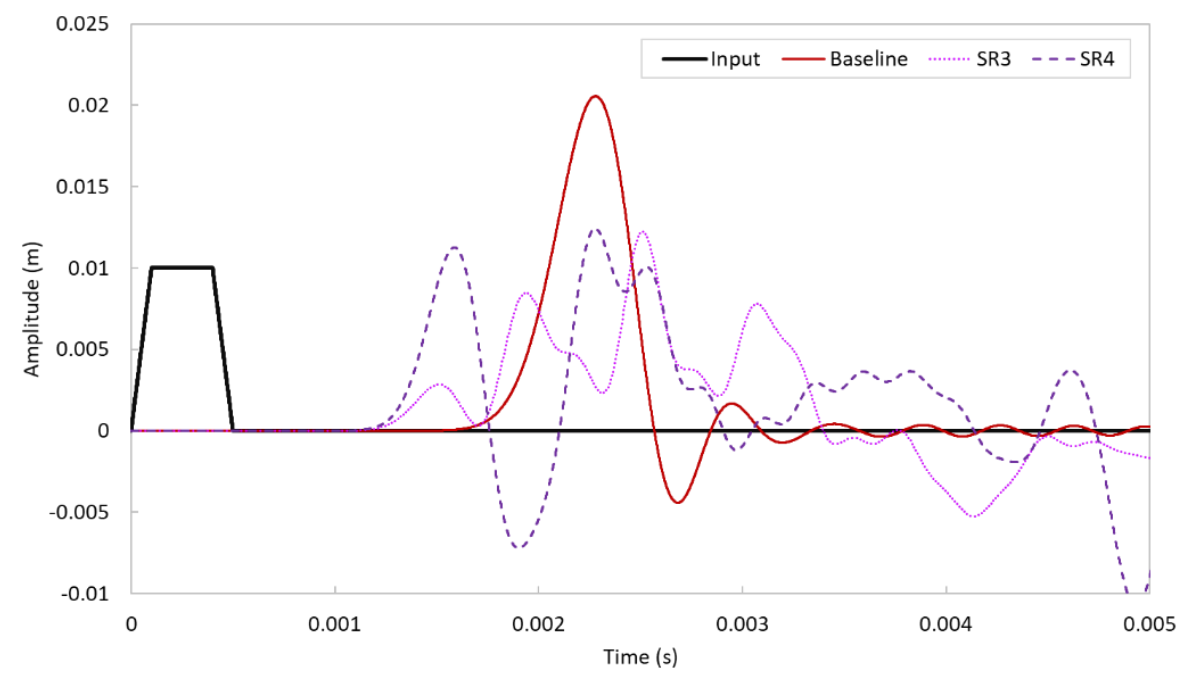

Figure 3.4: Input, baseline (SR7), and output displacement time histories for the two resonatortype cases.

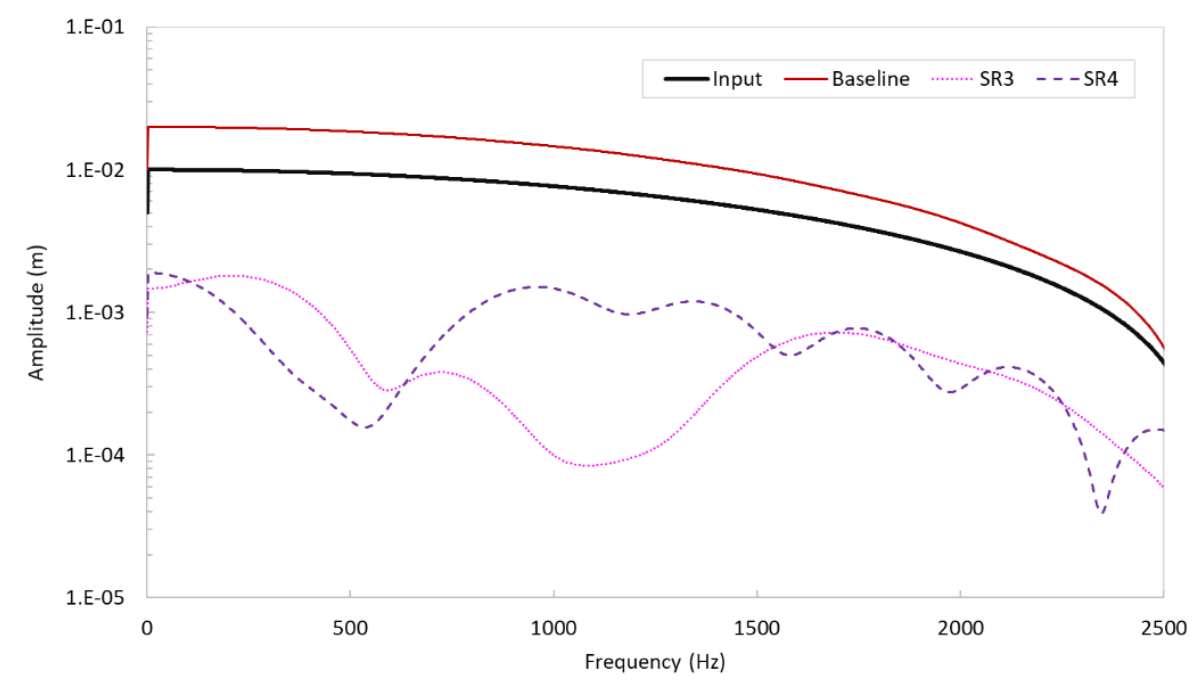

Figure 3.5: Input, baseline (SR7), and output displacement frequency spectra for the two resonator-type cases. 


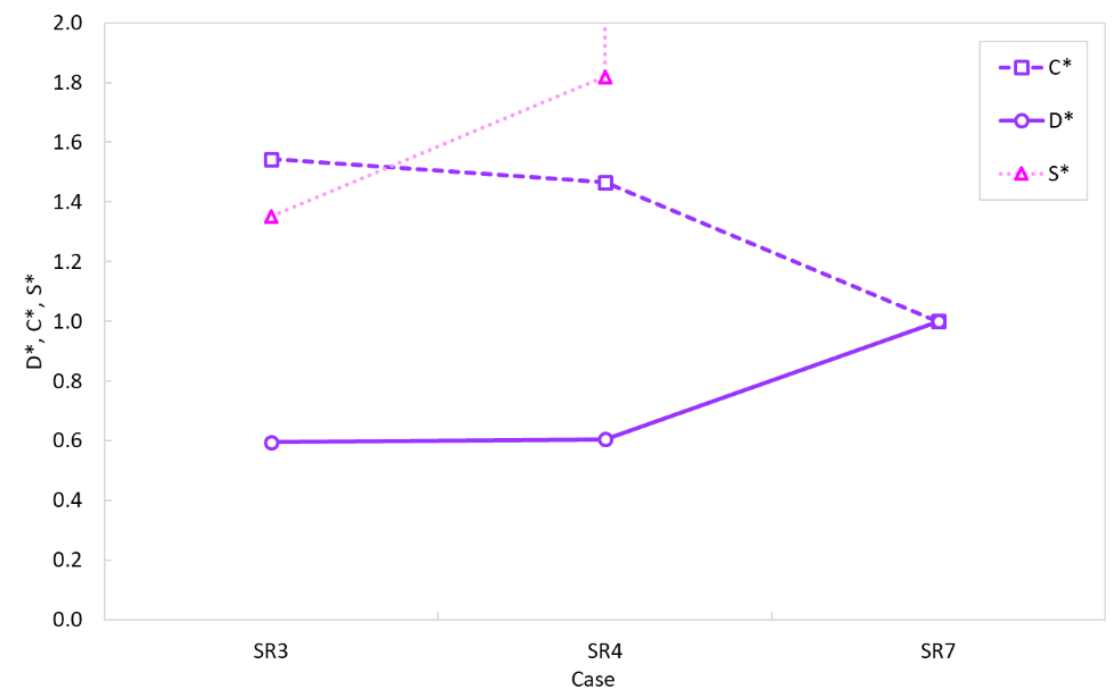

Figure 3.6: Output displacement ( $\left.D^{*}\right)$ and power spectral ( $\left.S^{*}\right)$ transmissibility and normalized wave speed $\left(C^{*}\right)$ for the two resonator-type cases.

\subsection{Four Resonator-Type Case}

Four resonator-type cases summarized in Table 3.3 are considered. The results are recapped in Figures 3.7, 3.8, and 3.9. Most of the cases are considerably yield a better D* value than the single or two resonator-type cases studied previously. SR14 is found to give the best D* result, this case corresponds to having an octave spacing, and the numbering of the resonators is proportional to the amplitude corresponding to its frequency in the input displacement spectrum. SR13 gives the second-best $D^{*}$ value; this case has four sets of octave spaced frequencies, with each set having an equal number of resonators. The octave spacing hypothesis remains to hold as both SR14 and SR13 gives the best D* performances for this case study. 


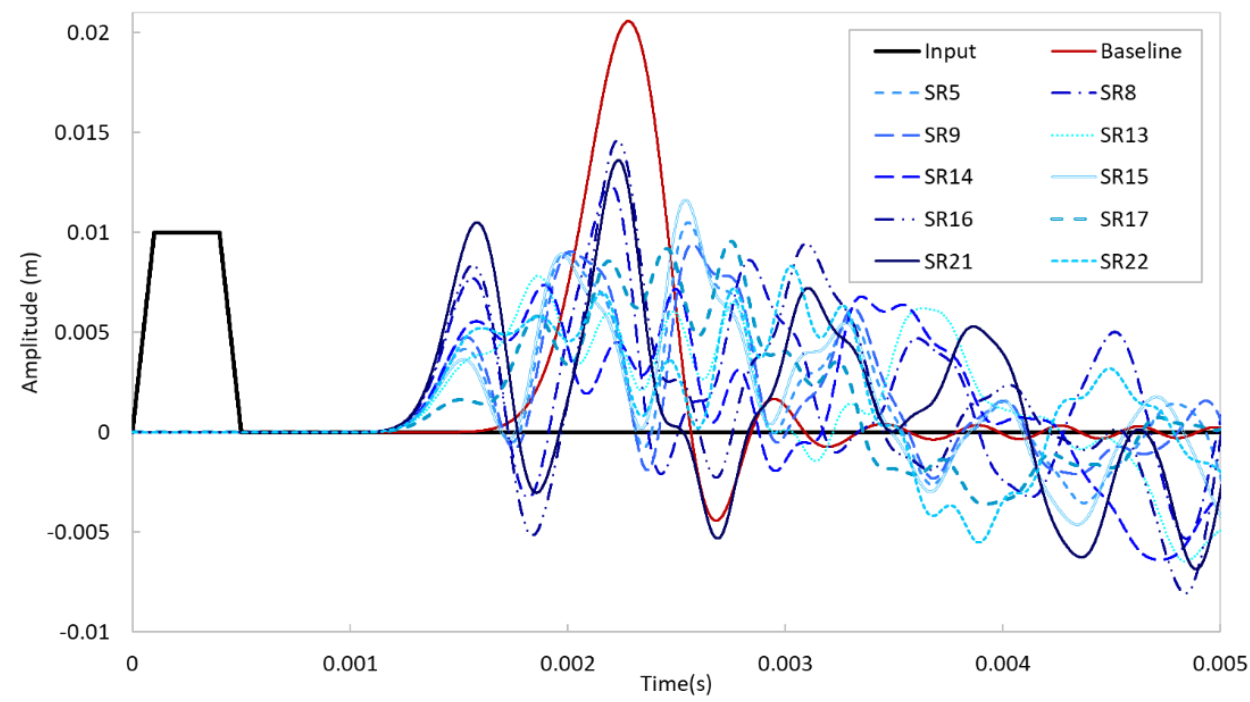

Figure 3.7: Input, baseline (SR7), and output displacement time histories for the four resonator-

type cases.

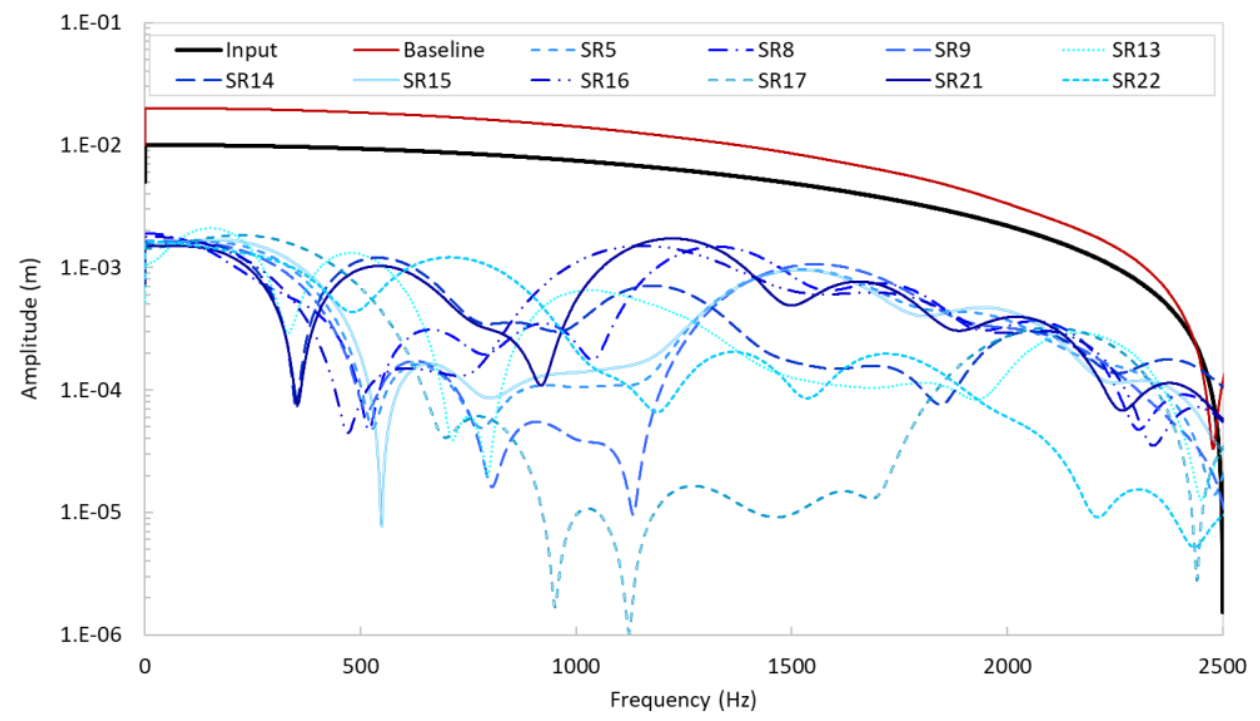

Figure 3.8: Input, baseline (SR7), and output displacement frequency spectra for the four resonator-type cases. 


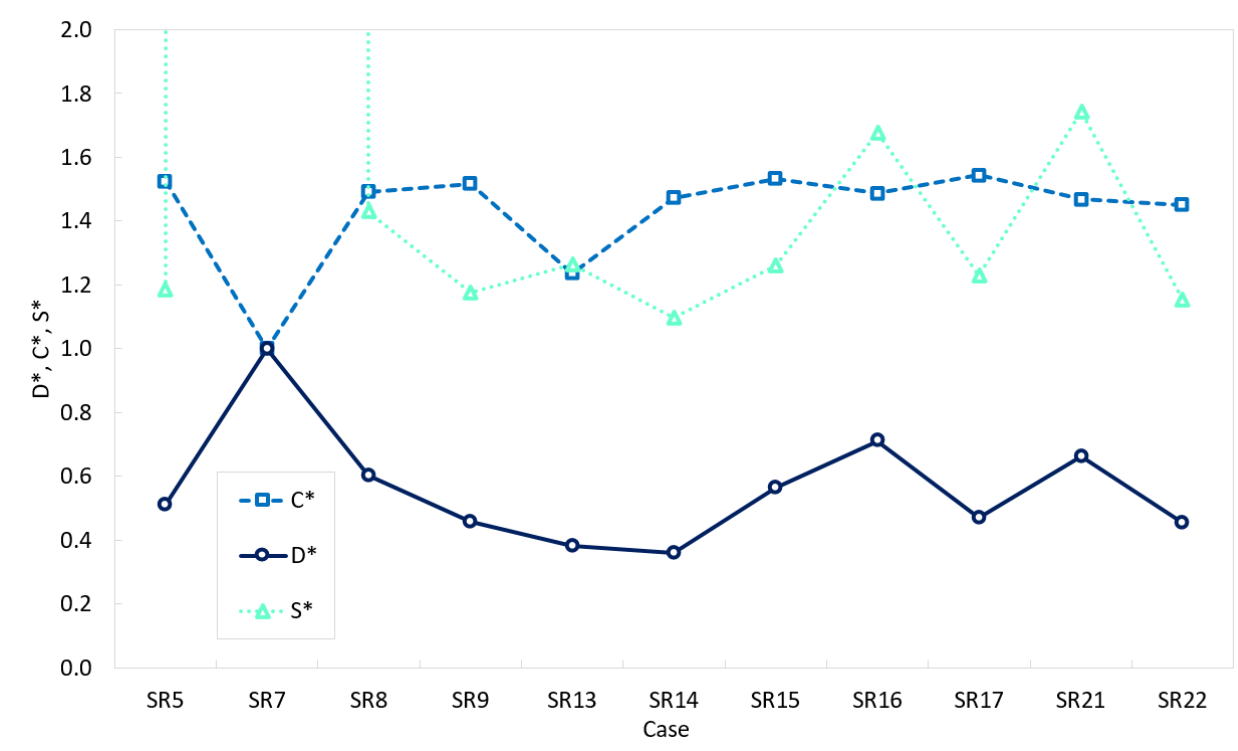

Figure 3.9: Output displacement $\left(D^{*}\right)$ and power spectral $\left(S^{*}\right)$ transmissibility and normalized wave speed $\left(C^{*}\right)$ for the four resonator-type cases.

This case's $\mathrm{D}^{*}$ value is also less than half of the baseline case. $\mathrm{S}^{*}$, which is based on the power spectral area, seems to follow more or less the same trend as D*, with SR14 remaining the best performing case for this metric too.

In general, amplitude proportional number of resonators and octave spacing is seen to outperform the evenly distributed resonator frequency cases (especially those at lower frequencies within the bandwidth of interest) as well as cases with coincident bandgaps. Therefore, this study provides an interesting insight on the selection of resonator frequencies for the AMB.

\subsection{N Distinct Resonator-Type Case}

The results are shown in Figures 3.10, 3.11 and 3.12. It is observed that evenly spaced $N$ distinct resonator-type cases do perform well but not as well as the best octave spacing case (SR14). Moreover, distributing the resonator frequencies over a higher frequency range within the bandwidth of interest is shown to give a relatively poorer performance. 


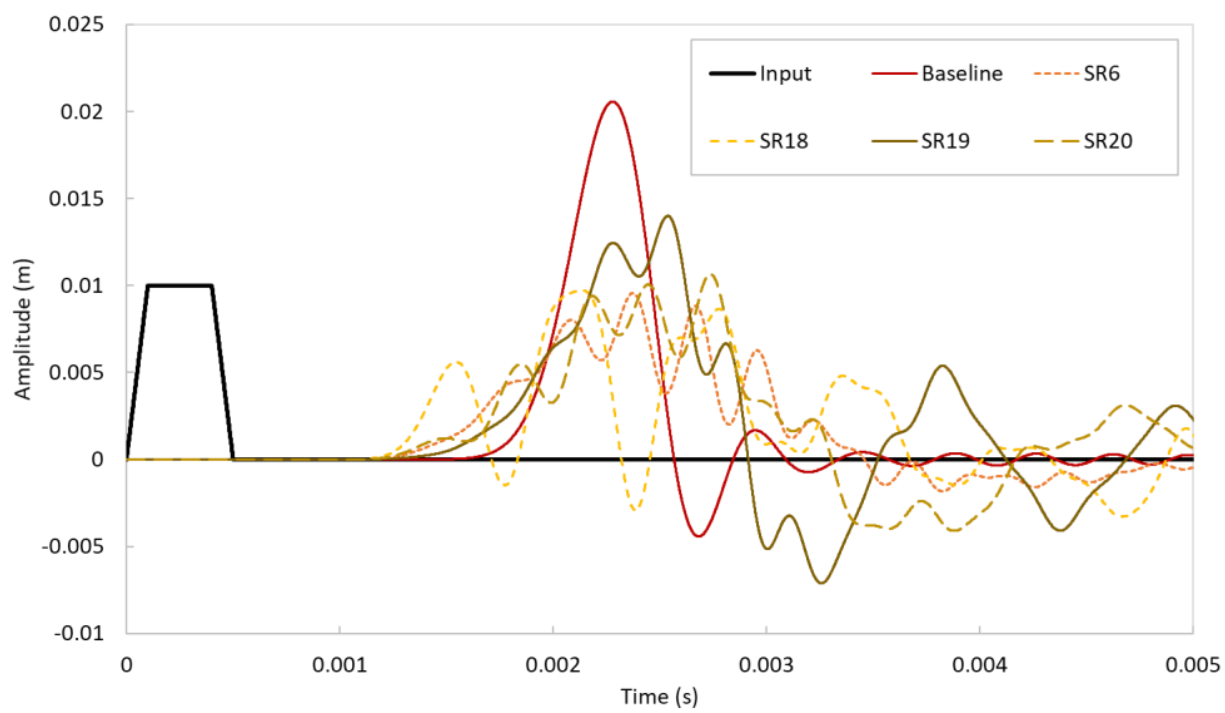

Figure 3.10: Input, baseline (SR7), and output displacement time histories for the $N(=20)$ distinct resonator-type cases.

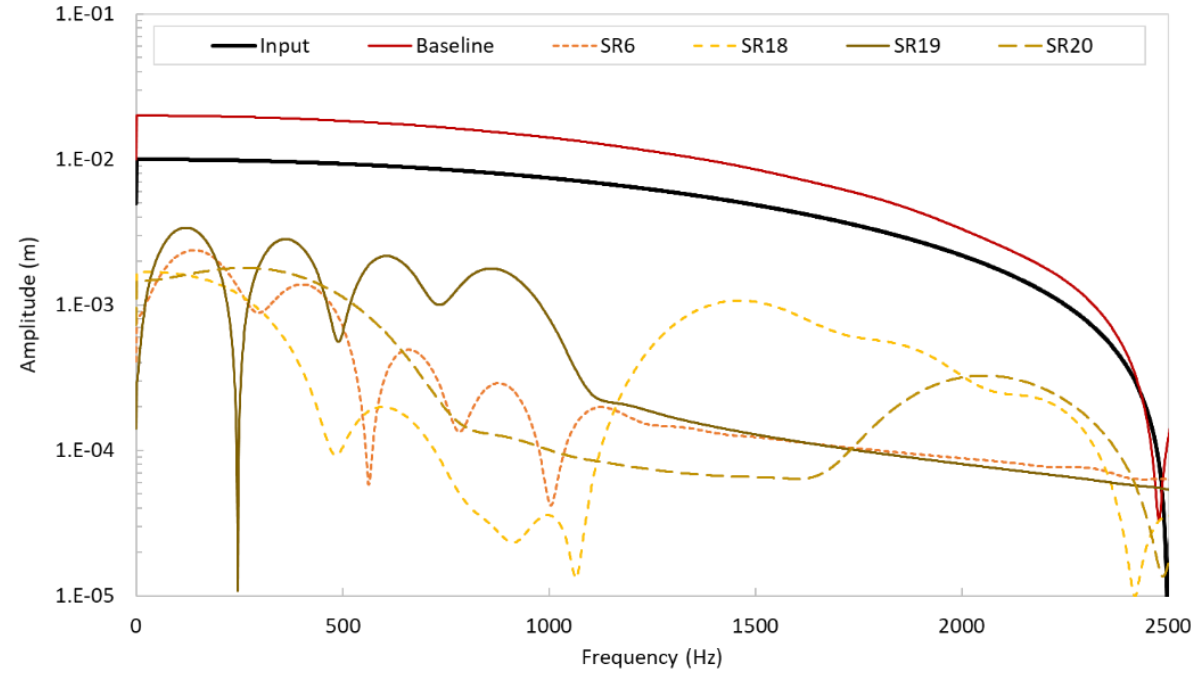

Figure 3.11: Input, baseline (SR7), and output displacement frequency spectra for the $N(=20)$ distinct resonator-type cases. 


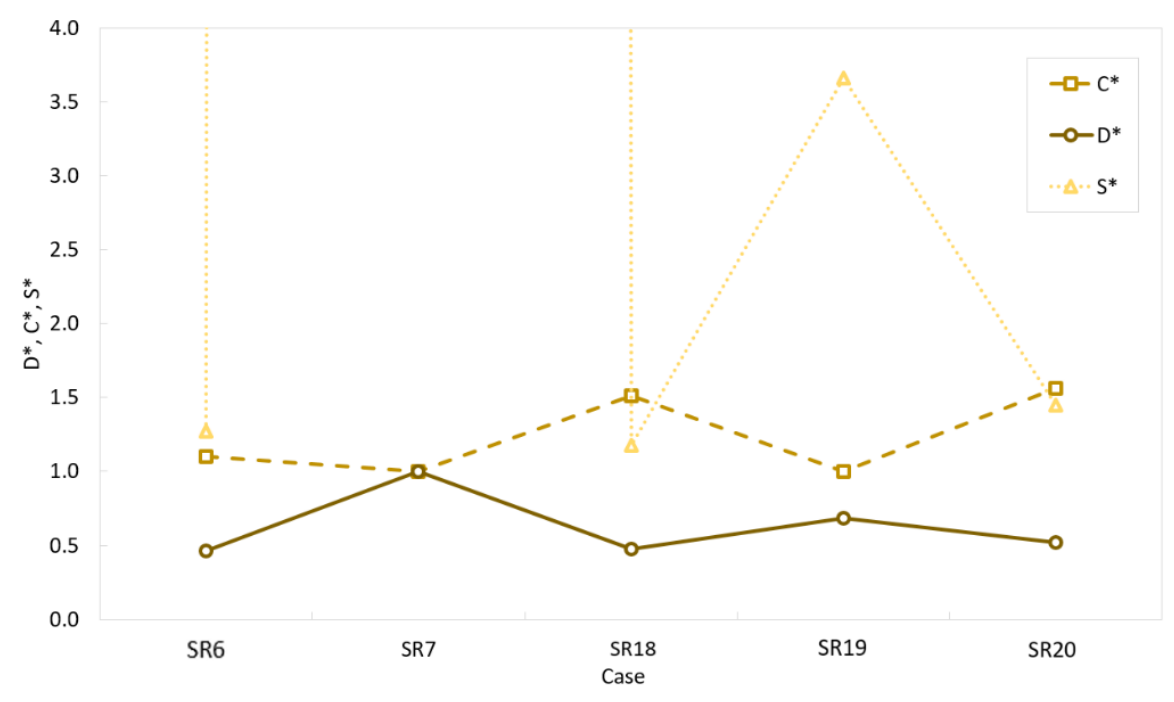

Figure 3.12: Output displacement $\left(D^{*}\right)$ and power spectral $\left(S^{*}\right)$ transmissibility and normalized wave speed $\left(C^{*}\right)$ for the four resonator-type cases.

\subsubsection{Resonator Sequence Variation Case}

In the resonator sequence variation (SQ) cases, the relative position of resonators within the best performing SR case (which is SR14) is varied. Many different approaches of sequencing are considered as summarized in Table 3.5. It is found that, in general, having the lowest frequency resonator set closest to the incident side gives the best performance while having higher frequency resonator sets first, mirroring, or fully interspersing the resonators of the best SR case all display poorer performance than cases with the lowest frequency resonator set first, which is another takeaway from this study. 


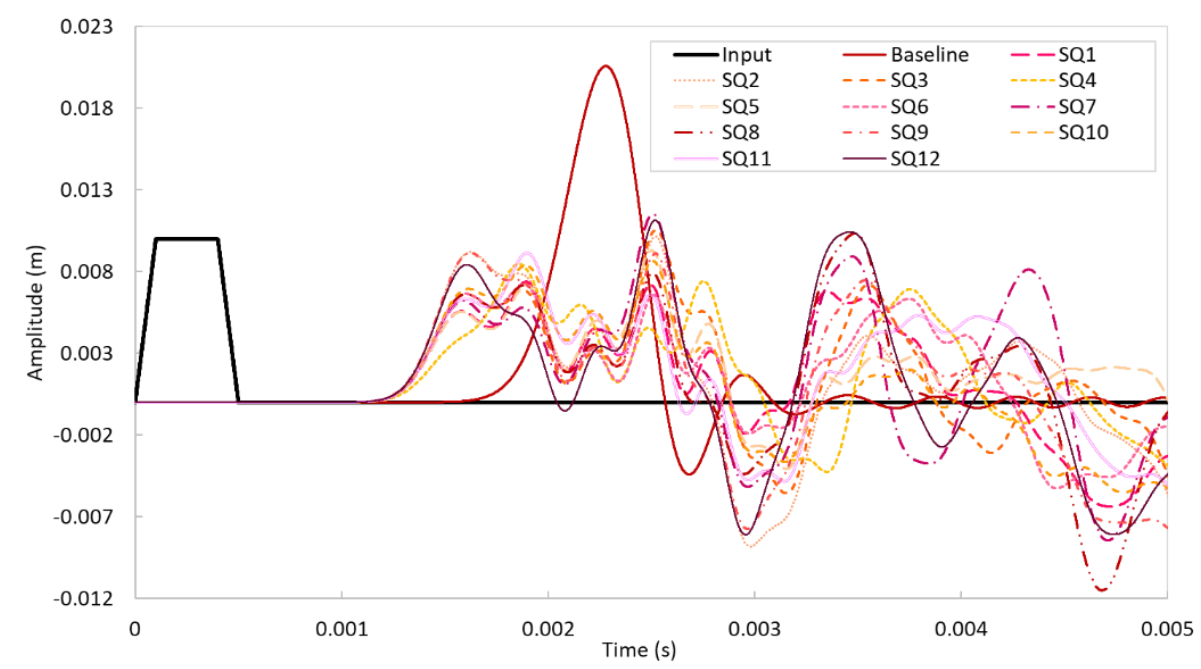

Figure 3.13: Input, baseline (SR7), and output displacement time histories for the resonator sequence variation cases.

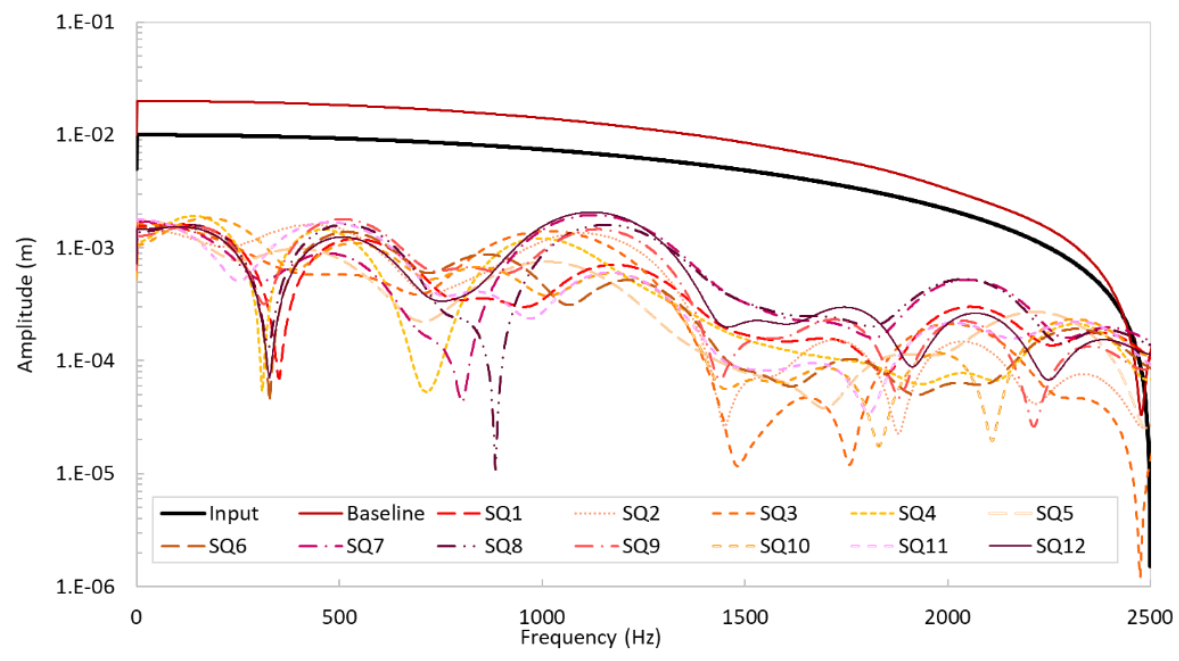

Figure 3.14: Input, baseline (SR7), and output displacement frequency spectra for resonator sequence variation cases. 


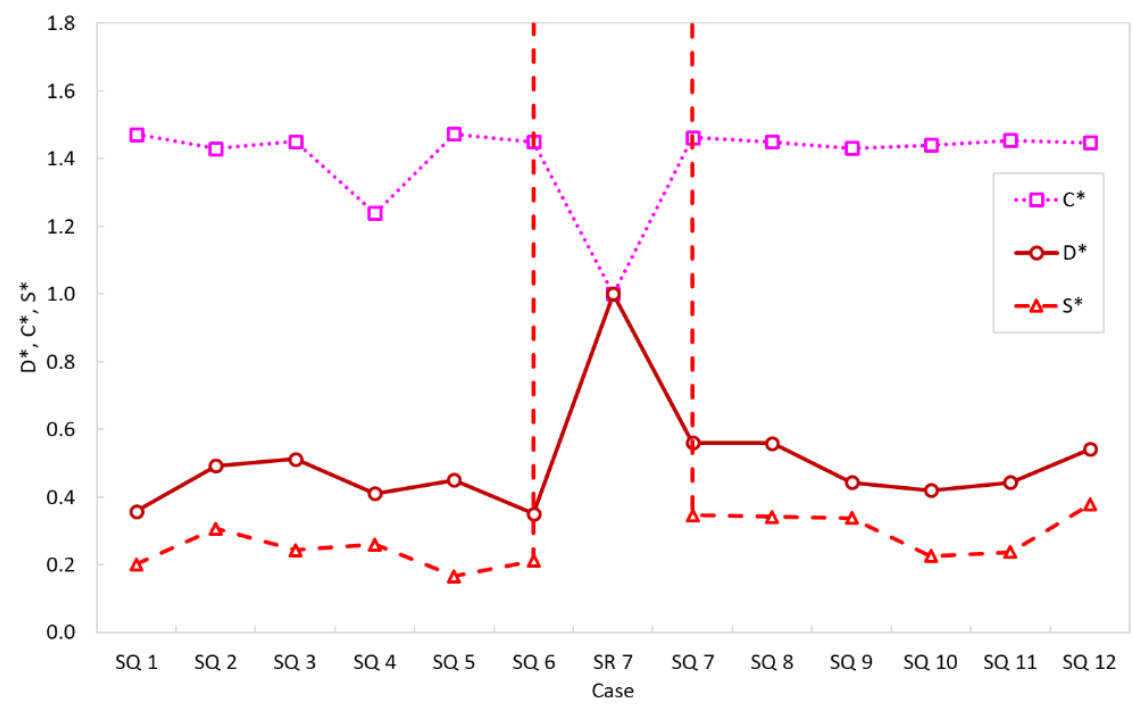

Figure 3.15: Output displacement $\left(D^{*}\right)$ and power spectral ( $\left.S^{*}\right)$ transmissibility and normalized wave speed $\left(C^{*}\right)$ for the resonator sequence variation cases.

The wave speed for the sequencing cases are seen to be more or less the same, given that the overall impedance of the waveguide is not affected much by the sequencing, although local changes may be induced.

\subsubsection{Material Variation Case}

The displacement time histories, spectra, and metrics are shown in Figures 3.16, 3.17, and 3.18. In parallel to the analogy inspired by the literary device of assonance, the influence of varying the host material's stiffness (similar to replacing consonants) on the targeted spectral transformation as specified by the metrics for the best performing sequence of resonators (similar to a set of vowels) is studied. Therefore, host material stiffness variation is considered for the SQ6 case. As described earlier, cases sequencing 'hard' and 'soft' host material stiffnesses in various patterns for units within the AMB are considered. 


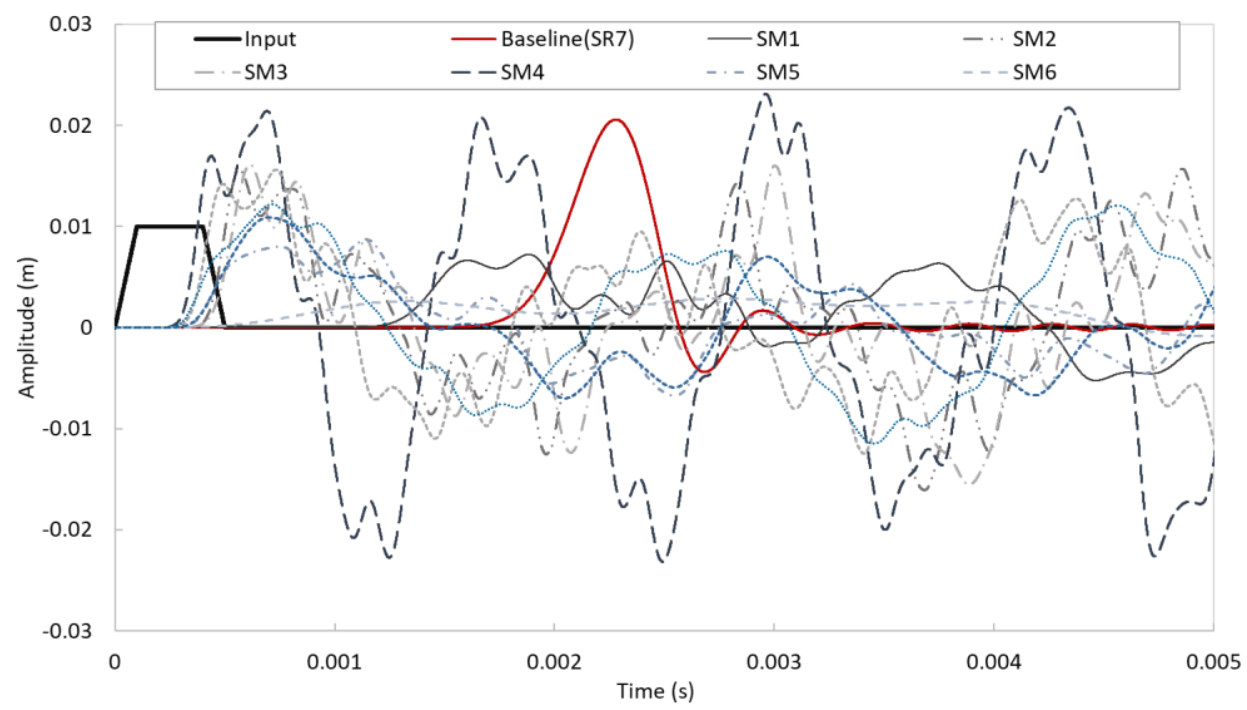

Figure 3.16: Input, baseline (SR7) and output displacement time histories for the host material stiffness variation cases.

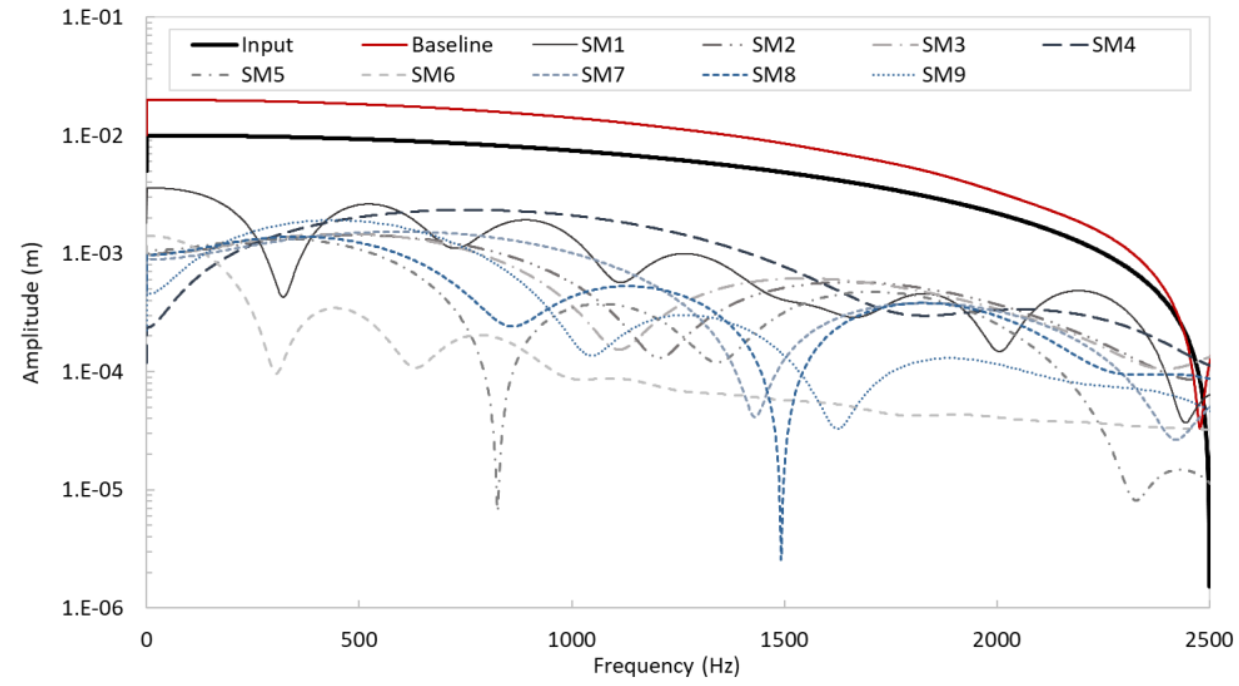

Figure 3.17: Input, baseline (SR7) and output displacement frequency spectra for the host material stiffness variation cases.

Overall, the best host material stiffness sequencing is found to be for SM6 in which a unit with 'soft' host material stiffness is sandwiched between two sets of units with identical 'hard' host 
material stiffness. This creates a high degree of impedance mismatch between the incident-side and the transmission-side of the AMB. The host material stiffness variation study shows that in addition to tailoring the resonator frequencies and their sequencing nearly $50 \%$ further reduction for $\mathrm{D}^{*}$ and nearly $75 \%$ further reduction for $\mathrm{S}^{*}$ can be achieved by an optimal arrangement of host material stiffness for the units within the AMB, thus illustrating the effectiveness of the assonant approach in the design of such metamaterial barriers.

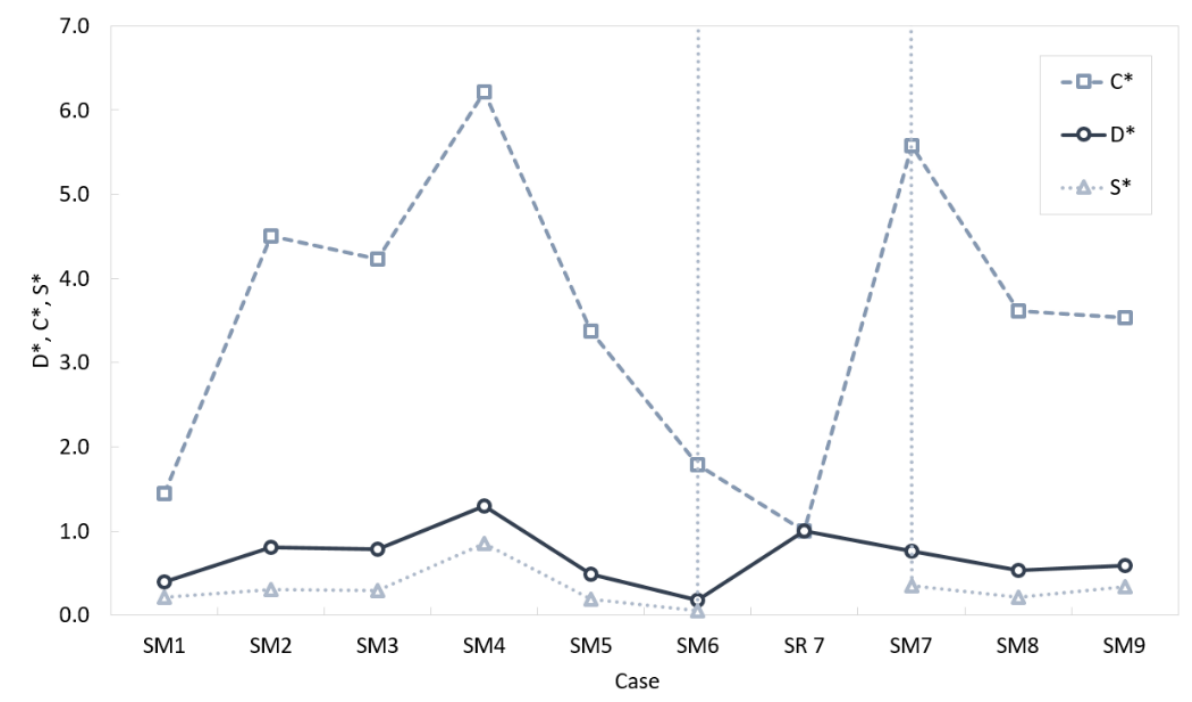

Figure 3.18: Output displacement $\left(D^{*}\right)$ and power spectral $\left(S^{*}\right)$ transmissibility and normalized wave speed $\left(C^{*}\right)$ for the host material stiffness variation cases.

It is noted that the static stiffness of the waveguide is retained remains unchanged for all cases. Since varying the host material stiffness induces impedance mismatch between units, large variation in normalized wave speed $\left(\mathrm{C}^{*}\right)$ is observed for these cases.

\section{$\underline{3.3 \text { Summary }}$}

A numerical exploration for an acoustic metamaterial barrier (AMB) subjected to a broadband input is carried out to discover relative mechanical wave mitigation performance based on transmissibility metrics. As a measure of equivalence between the baseline parameter and the 
various $A M B$ cases considered in this study, the static mass and stiffness is retained invariant for all cases. Based on the results of this study, the following conclusions are made:

- The attenuation performance of the AMB can be improved by choosing the number of resonators for each set to be proportional to the amplitude corresponding to its frequency in the input (step) displacement spectrum.

- Simultaneously, it is also found that using an octave spacing for the specific frequencies chosen for resonator sets yielded the best result among the resonator frequency variation cases considered. Amongst the cases considered in this study, the four resonator-type cases outperformed both single resonator-type cases and the $N$ distinct resonator-type cases.

- Distributing the resonator frequencies over a higher frequency range within the bandwidth of interest is shown to give a relatively poorer performance.

- From a sequencing perspective it is found that having the low frequency resonator placed near the incident side gives the best performance.

- Engineering a high degree of impedance mismatch by sandwiching a unit with relatively 'soft' host material stiffness between two sections of units with relatively 'hard' host material stiffness enhances the overall attenuation performance provided by resonator action.

From a practical perspective, assonant mechanisms are found to play a significant role in determining the attenuation performance of an $\mathrm{AMB}$ when design constraints relevant to applications such as prescribed geometry, material, number of resonators, and number of sets of resonator frequencies are considered. In a similar vein, the term 'mechanical assonance' can be ascribed to the dynamic phenomenon realized by sequencing oscillators with tuned responses within a waveguide to engineer a prescribed wave transformation across it. 


\section{CHAPTER IV}

\section{IMPACT ENERGY HARVESTING USING ASSONANT ACOUSTIC METAMATERIAL}

\subsection{Introduction}

This chapter investigates the energy harvesting application for a mechanically assonant acoustic metamaterial. Energy can never be destroyed; however, it is possible to convert one form into another. For instance, the vibration energy sequestered within local attachments of an AM can be converted to useable electrical energy by applying an electromechanical coupling. This study is motivated by the possibility of having structural materials that could improve the efficacy and utility of built structures in adverse environmental conditions.

\subsection{Translational EMF Derivation}

Figure 4.1 depicts a magnet constrained to move only along the z-axis, surrounded by a loop of wires that are able to harvest energy. A change in the magnetic flux around coils will induce a change in current in each wire loop, thus generating EMF. From the Lorentz force law, one can easily derive Faraday's Equation, which is given by:

$$
\varepsilon=-N_{\text {coils }} \frac{\mathrm{d} \Phi}{\mathrm{d} t}
$$



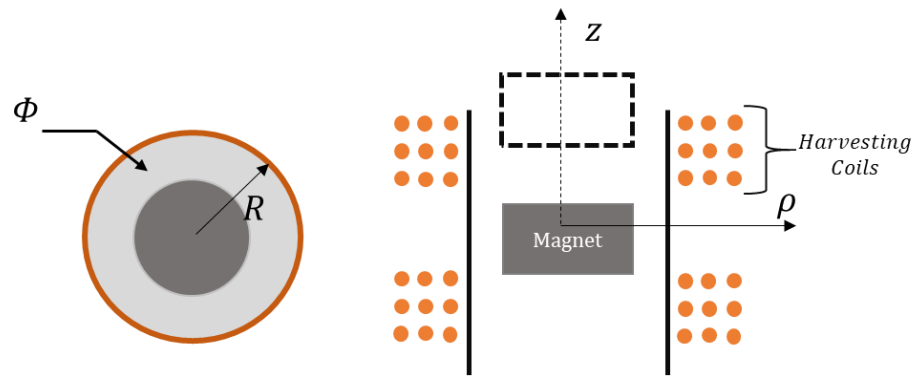

Figure 4.1: A simple depiction of an oscillating magnet along the axial direction surrounded by coils.

Where $\varepsilon$, is the electromotive force in Volts $(\mathrm{V}), N_{\text {coils }}$ is the number of coils around the magnet, and $\Phi$ is the magnetic flux. The negative sign comes from the fact the EMF generates a current with its magnetic field that opposes the change in flux; this phenomenon is known as Lenz's law. The magnetic flux is a measure of the amount of magnetic field passing through a surface and is given by:

$$
\Phi=B \cdot A
$$

Equation (4.2) shows that the magnetic flux is a measure of the total magnetic field passing through a given area. The flux is maximum when the area is perpendicular to the field and minimum when it is parallel to the field. Therefore, we will be using the magnetic field along the axial direction, $B_{z}$ due to an assumption that the magnet is only restricted to move along the z-axis furthermore, the flux generated by the radial magnetic fields, $B_{R}$ will be zero because the field is parallel to the wires. The change in magnetic flux is caused by the motion of the magnet along the axial direction, as seen in Equation (4.3) below:

$$
\frac{\mathrm{d} \Phi}{\mathrm{d} t}=\frac{\mathrm{d} B_{Z}}{\mathrm{~d} t} \cdot A
$$

Applying the chain rule Equation (4.4) can be derived as:

$$
\frac{\mathrm{d} \Phi}{\mathrm{d} t}=\frac{\mathrm{d} B_{Z}}{\mathrm{~d} z} \cdot \frac{\mathrm{d} z}{\mathrm{~d} t} \cdot A
$$


where, $\frac{\mathrm{d} z}{\mathrm{~d} t}$ is the velocity of the magnet along the $\mathrm{z}$-axis.

Combining Equation (4.1) and (4.4), we can get:

$$
\varepsilon_{\text {co-located }}=N_{\text {coils }} \frac{\mathrm{d} B_{z}}{\mathrm{~d} z} \cdot \frac{\mathrm{d} z}{\mathrm{~d} t} \cdot A
$$

Equation 4.5 assumes that all the coils are co-located at the center of the magnet from a non-zero radial distance; this assumption might affect the Equation accuracy. However, using the derived magnetic fields, and by defining each coil position, it is possible to calculate the induced EMF in each coil and by taking the sum to get the overall harvested voltage. This Equation may be given as:

$$
\varepsilon_{\text {distributed }}=\sum_{i=1}^{n} \sum_{j=1}^{m}\left[\frac{\mathrm{d} B_{z}}{\mathrm{~d} z}\right]_{i} \cdot A_{j} \cdot \frac{\mathrm{d} z}{\mathrm{~d} t}
$$

In Equation 4.6, the inner sigma notation that runs with index, ' $j$ ' sums the harvested voltage for each coil positioned along the radial direction. For each coil located in the radial direction, an index ' $i$ ' adds along the axial direction to get the total EMF.

\subsection{Magnetic Field Derivation}

To derive the magnetic field created by a cylindrical magnet shown in Figure 4.2, which can be computed directly from the Biot-Savart law:

$$
B=\iiint \frac{\mu_{0} \mathrm{M}}{4 \pi} \frac{d r^{\prime} \times\left(\vec{r}-\vec{r}^{\prime}\right)}{\left|\vec{r}-\vec{r}^{\prime}\right|} \mathrm{d} \vec{V}
$$

Where, $\mu_{0}$ is the permeability of free space, $\mathrm{M}$ is magnetization, $d r^{\prime}$ the infinitesimal step is taken along the radial position, $\vec{r}$ and $\vec{r}^{\prime}$ represent the distance from the origin to a magnetic field in space and to an infinitesimal dipole moment, $\mathrm{d} m$. In Figure 4.2, the coordinate points $\rho^{\prime}, \psi^{\prime}, z^{\prime}$ 
represent the position of the dipole moment, $m$ from the origin. The radius and length of the magnet are defined as $\mathrm{R}$ and $\mathrm{L}$, respectively. To begin the derivation by assuming uniform dipole moment and radial symmetry of the cylinder about the z-axis.

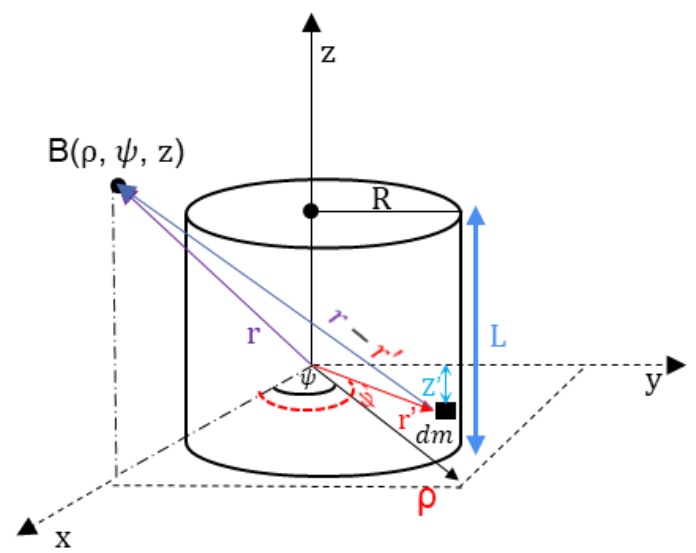

Figure 4.2: Schematic depiction of a finite magnetized cylinder.

The position vector, $\vec{r}^{\prime}$ can be written as:

$$
r^{\prime}=R \cos \left(\psi^{\prime}\right) \hat{\boldsymbol{\imath}}+R \sin \left(\psi^{\prime}\right) \hat{\boldsymbol{j}}+z^{\prime} \widehat{\boldsymbol{k}}
$$

taking the derivative to get the infinitesimal step

$$
\mathrm{d} r^{\prime}=-\left((R \sin (\psi) \hat{\boldsymbol{\imath}}-R \cos (\psi) \hat{\boldsymbol{\jmath}}) \mathrm{d} \psi^{\prime}\right.
$$

Because of the cylindrical symmetry, $\mathrm{r}$ can be written as $\mathrm{r}=\rho \hat{\boldsymbol{\imath}}+z \widehat{\boldsymbol{k}}$. Then the distance from a dipole moment to any magnetic field in space can be defined as:

$$
r-r^{\prime}=\left(\rho-R \cos \left(\psi^{\prime}\right)\right) \hat{\boldsymbol{\imath}}-R \sin \left(\psi^{\prime}\right) \hat{\boldsymbol{\jmath}}+\left(z-z^{\prime}\right) \widehat{\boldsymbol{k}}
$$

and

$$
\mathrm{d} r^{\prime} \times\left(r-r^{\prime}\right)=R\left[\left(\left(z-z^{\prime}\right) \cos \psi^{\prime}\right) \hat{\boldsymbol{\imath}}+\left(\left(z-z^{\prime}\right) \sin \psi^{\prime}\right) \hat{\boldsymbol{\jmath}}+\left(R-\cos \psi^{\prime}\right) \widehat{\boldsymbol{k}}\right] d \psi
$$

The $B_{Z}$ field can be written as:

$$
B_{Z}(\rho, z)=\int_{-\frac{l}{2}}^{\frac{l}{2}} \mathrm{dz} z^{\prime}\left(\frac{\mu_{0} M R}{2 \pi}\right) \int_{0}^{\pi}\left(\frac{\left(\left(z-z^{\prime}\right) \cos \psi^{\prime}\right) \hat{\boldsymbol{\imath}}+\left(R-\rho \cos \psi^{\prime}\right) \widehat{\boldsymbol{k}}}{\left[\rho^{2}-2 R \rho \cos \psi^{\prime}+R^{2}+\left(z-z^{\prime}\right)^{2}\right]^{3 / 2}}\right) \mathrm{d} \psi
$$

and $B_{\rho}$ as: 


$$
B_{\rho}(\rho, z)=\frac{\mu_{0} M}{2 \pi} \int_{-\frac{l}{2}{ }_{-}}^{\frac{l}{2}} \int_{0}^{\pi} \frac{\left(z-z^{\prime}\right) \cos \psi^{\prime}}{\left[R^{2}+\rho^{2}-2 R \rho \cos \psi^{\prime}+\left(z-z^{\prime}\right)^{2}\right]^{3 / 2}}
$$

Now, performing elementary integration for both $B_{Z}$ and $B_{\rho}$ along $z^{\prime}$ gives:

$$
\begin{gathered}
B_{z}(\rho, z)=\frac{\mu_{0} M}{2} \int_{0}^{\pi} \frac{\left(R-\rho \cos \psi^{\prime}\right)}{\left(\rho^{2}+R^{2}-2 R \rho \cos \psi^{\prime}\right)}\left[\frac{\sqrt{\left(z+\frac{l}{2}\right)^{2}+\rho^{2}+R^{2}-2 R \rho \cos \psi^{\prime}}}{\sqrt{\left(z-\frac{l}{2}\right)^{2}+\rho^{2}+R^{2}-2 R \rho \cos \psi^{\prime}}}\right] \mathrm{d} \psi \\
B_{\rho}(\rho, z)=-\frac{\mu_{0} M}{2} \int_{0}^{\pi}\left[\frac{1}{\sqrt{\left(z+\frac{l}{2}\right)^{2}+\rho^{2}+R^{2}-2 R \rho \cos \psi^{\prime}}}-\frac{1}{\sqrt{\left(z-\frac{l}{2}\right)^{2}+\rho^{2}+R^{2}-2 R \rho \cos \psi^{\prime}}}\right] \cos \psi^{\prime} \mathrm{d} \psi
\end{gathered}
$$

To simplify the Equation, let's define some terms as $Z_{ \pm}=z \pm \frac{l}{2}$ and $\beta=\rho^{2}+R^{2}-2 R \rho \cos \psi^{\prime}$

Equation 4.13 can be rewritten as:

$$
B_{z}(\rho, z)=\frac{\mu_{0} M}{2} \int_{0}^{\pi} \frac{\left(R-\rho \cos \psi^{\prime}\right)}{\beta}\left[\frac{z_{+}}{\sqrt{z_{+}{ }^{2}+\beta}}-\frac{z_{-}}{\sqrt{z_{-}^{2}+\beta}}\right] \mathrm{d} \psi
$$

Now, inserting Equation 4.6 into equation 4.15 gives:

$\varepsilon_{\text {distributed }}=\sum_{i=1}^{n} \sum_{j=1}^{m} B_{o} \cdot \rho_{j}{ }^{2} \cdot \frac{d z}{d t} \cdot \frac{R-\rho j \cos \psi}{\beta} \int_{0}^{\pi}\left[\frac{1}{\left(z_{+}{ }^{2}+\beta_{j}\right)^{\frac{1}{2}}}-\frac{1}{\left(z_{-}{ }^{2}+\beta_{j}\right)^{\frac{1}{2}}}+\frac{2 \cdot z_{-}}{\left(2 \cdot z_{-}{ }^{2}+\beta_{j}\right)^{\frac{3}{2}}}-\frac{2 \cdot z_{+}}{\left(2 \cdot z_{+}{ }^{2}+\beta_{j}\right)^{\frac{3}{2}}}\right] \mathrm{d} \psi(4.16)$

Where $B_{o}=\frac{\mu_{0} M R}{2}$. The sigma notations in Equation (4.16) replace the multiplication factor $N$ for the number of coils and instead measure and sums the EMF generated in each coil along with the axial and radial directions. The integration along $\psi$ in Equation (4.14 and 4.15) is difficult to compute analytically; therefore, a numerical method of computation was provided by Derby and Olbert [49], the translated MATLAB version of the code included in Appendix A.6. 


\section{$\underline{\text { 4.4 Translational EMF Study }}$}

For maximum power output, vibration-based energy harvesters are commonly engineered to exhibit natural frequencies that resonate with the ambient source excitation. The design of such a device is shown in Figure 4.3 below. A cylindrical neodymium magnet is used as the internal mass, which is the multifunctional element. Each side of the magnet is bonded to the ends of a compression spring by a cyanoacrylate adhesive. The magnet-spring assembly form the inner resonator unit. The resonator casing, caps, and shaker adapter are fabricated from a 3D printed polymer material. Twenty turns of enamel coated wires are stacked along the resonator's length and are placed inside a coil housing. The damping constant was retrieved by applying the log decrement method on a displacement time history data collected from a tap test.

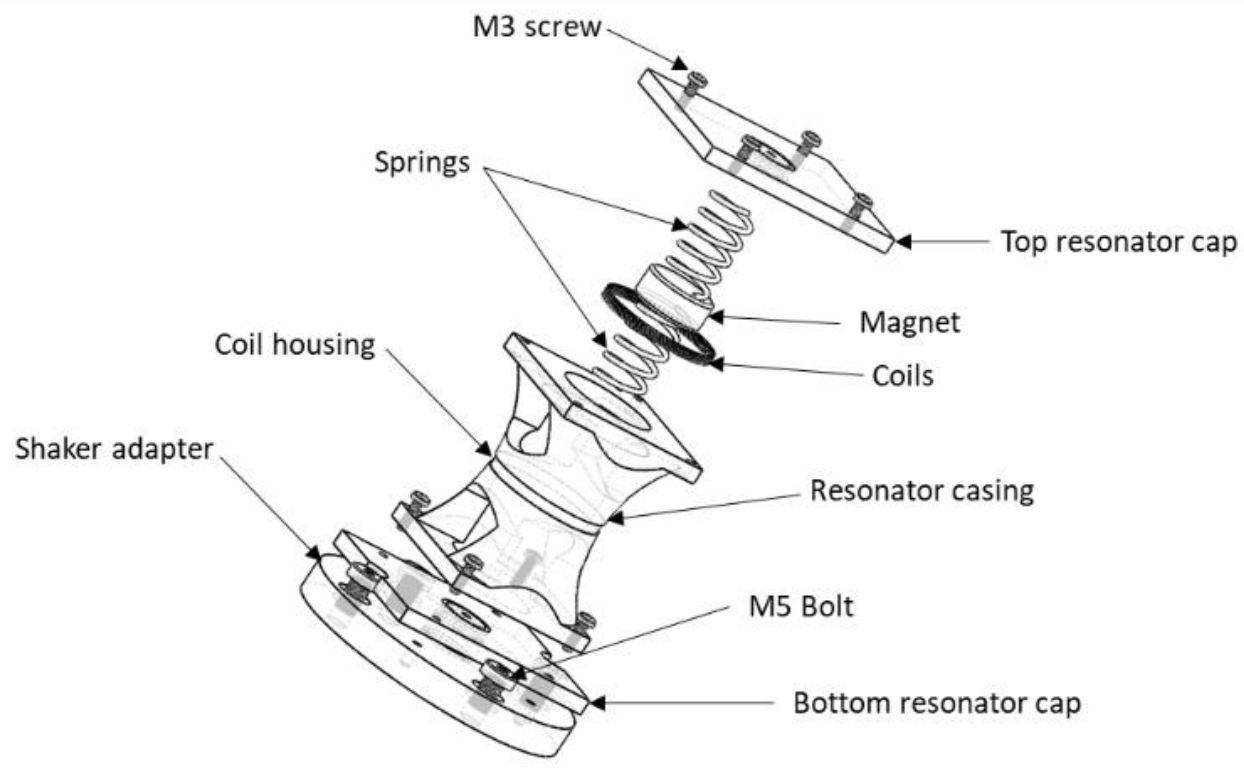

Figure 4.3: Exploded view of the harvesting device. 
Table 4.1: Summary of base parameters used for EMF model verification.

\begin{tabular}{|c|c|c|c|c|}
\hline & Description & Symbol & Value & Unit \\
\hline \multirow{5}{*}{ 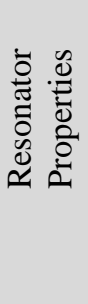 } & $1.75 \mathrm{~mm}$ PLA filament & - & 1240 & $\mathrm{~kg} / \mathrm{m}^{3}$ \\
\hline & Spring stiffness & $k$ & 1595 & $\mathrm{~N} / \mathrm{m}$ \\
\hline & Damping value & $c_{d}$ & 0.23 & N.s/m \\
\hline & Resonator frequency & $f_{0}$ & 54 & $\mathrm{~Hz}$ \\
\hline & Coil Position & - & 19 & $\mathrm{~mm}$ \\
\hline \multirow{4}{*}{ 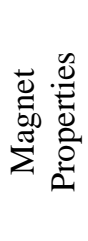 } & Magnet mass & $m$ & 0.0277 & $\mathrm{~kg}$ \\
\hline & Magnet length & $l_{m}$ & 0.009525 & $\mathrm{~m}$ \\
\hline & Magnet radius & $R_{m}$ & 0.0111125 & $\mathrm{~m}$ \\
\hline & Magnet dipole moment & $m_{d}$ & 3.88 & A. $m^{2}$ \\
\hline
\end{tabular}

\subsubsection{Definition of Test Cases}

The EMF study verification begins with the definition of three categories of test cases that depend on the harvesting coils' position relative to the magnet. These test cases will be made for two models, theoretical and semi-analytical. The coil positioning formats are stated as co-located, mirror, and interspersed configurations, which are depicted in Figure 4.4. This set of test cases may give us insight into optimum coil placement and verify the EMF code. Co-located coil assumes that all harvesting wires will experience the same magnetic field variation; therefore, the total EMF is calculated as described in Equation 4.5. The mirror coil configuration assumption takes in consideration that the two sets of harvesting wires are symmetrical to one another, and thus it is possible to assume that each harvesting wires are equidistant above and below the origin, therefore effectively experiencing equal and opposite magnetic field. 


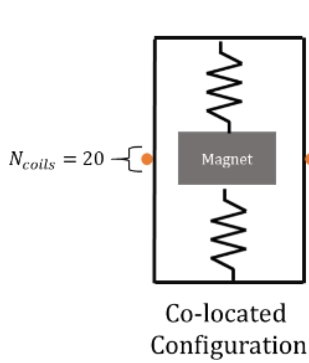

(a)

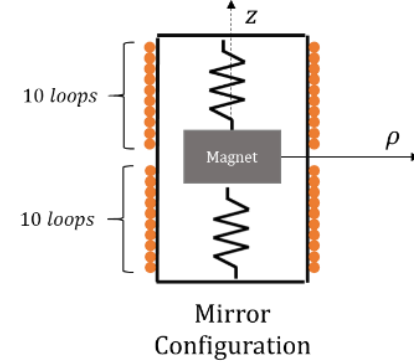

(b)

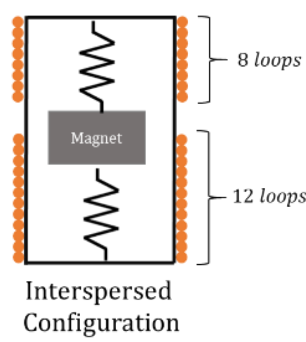

(c)

Figure 4.4: Schematic depiction for (a) co-located, (b) mirror, and (c) interspersed

$$
\text { configuration. }
$$

For the mirror configuration, two sets of harvesting coils are assumed to be located $1 \mathrm{~mm}$ from the center of the magnet along its vertical axis; each loop of wire is made from 10 turns placed above the center, whereas the other ten wires are positioned below. During the assembly of the harvester device, the pair of coils had an uneven number of loops, with only eight sets of wires located above the center, while the ones below had twelve; for this randomness, this configuration is defined as interspersed coils.

\subsubsection{Theoretical Model}

A simple spring-mass model shown in Figure 4.5 with a base excitation boundary condition and a fixed end is used to depict the experiment model. The dashpot represents the overall damping within the system.

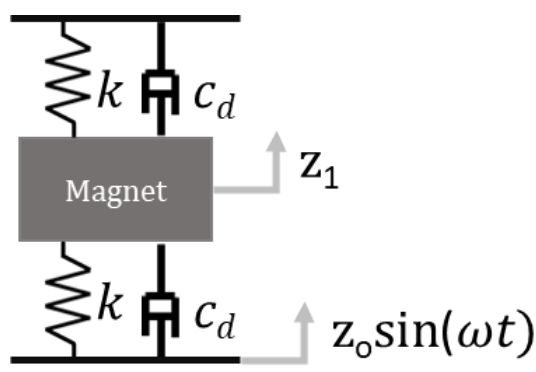

Figure 4.5: Spring-mass model representation of the electromechanically coupling. 


\section{Abaqus \\ simulation \\ Displacement \\ response \\ EMF code}

Figure 4.6: Theoretical model data flowchart.

Table 4.2: Summary of test case studies for the proposed theoretical model.

\begin{tabular}{|c|c|c|c|}
\hline & \multicolumn{3}{|c|}{ Theoretical Model } \\
\hline & Test Cases & $\begin{array}{c}\text { Excitation } \\
\text { Frequency } \\
\quad(\mathrm{Hz})\end{array}$ & $\begin{array}{c}\text { Input } \\
\text { Displacement } \\
\quad(\mathbf{m m})\end{array}$ \\
\hline \multirow{4}{*}{ 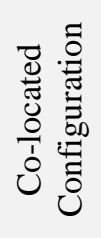 } & TCo-52 & 52 & 0.07 \\
\hline & TCo-54 & 54 & 0.015 \\
\hline & TCo-56 & 56 & 0.07 \\
\hline & TCo-58 & 58 & 0.07 \\
\hline \multirow{4}{*}{$\dot{\overbrace{}}$} & TMC-52 & 52 & 0.07 \\
\hline & TMC-54 & 54 & 0.015 \\
\hline & TMC-56 & 56 & 0.07 \\
\hline & TMC58 & 58 & 0.07 \\
\hline \multirow{4}{*}{ 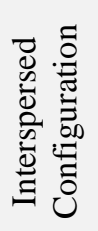 } & TIC-52 & 52 & 0.07 \\
\hline & TIC-54 & 54 & 0.015 \\
\hline & TIC-56 & 56 & 0.07 \\
\hline & TIC-58 & 58 & 0.07 \\
\hline
\end{tabular}

The assumptions made for this study are:

- Attached or internal mass (magnet) is restricted to move only along the z-axis.

- Damping constant is assumed to be the same for all frequency case studies.

- Back EMF effects are not considered.

- Heat and eddy current losses within the harvesting wires are neglected.

- All assumptions considered in the derivation of the field equation will apply here. 
The theoretical model study will aid with the understanding of the dynamic system in ideal conditions. The theoretical EMF is calculated by retrieving the displacement time history from the results of a numerical study conducted in Abaqus and by plugging it into the EMF code, as shown in Figure 4.6.

\subsubsection{Semi-Analytical Model}

A semi-analytical model is introduced, which utilizes the magnet's response and base excitation data collected using the laser vibrometer and accelerometer during the experiment that will be used to calculate the theoretical harvested voltage. Semi-analytical EMF data can be obtained by utilizing the relative displacement into the EMF code. The relative displacement calculates the difference between the magnet's response to that of the base excitation. The laser vibrometer and accelerometers can only output velocity and acceleration time histories, respectively. Therefore, numerical integration is used to extract displacement time history from acceleration and velocity.

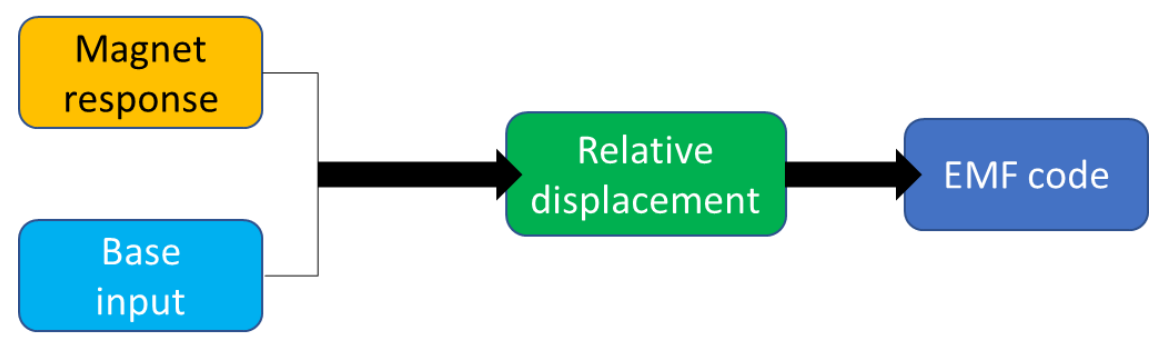

Figure 4.7: Semi-analytical model data flowchart.

Assumptions considered for the semi-analytical study are listed below:

- Back EMF is not considered for this model study.

- Heat and eddy current losses within the harvesting wires are neglected.

- All assumptions considered in the derivation of the field equation will apply here. 
Table 4.3: Summary of test case studies for the proposed semi-analytical model.

\begin{tabular}{|c|c|c|c|}
\hline & \multicolumn{3}{|c|}{ Semi-Analytical Model } \\
\hline & $\begin{array}{l}\text { Test } \\
\text { Cases }\end{array}$ & $\begin{array}{c}\text { Excitation } \\
\text { Frequency } \\
\text { (Hz) }\end{array}$ & $\begin{array}{c}\text { Input } \\
\text { Displacement } \\
(\mathbf{m m})\end{array}$ \\
\hline \multirow{4}{*}{$\begin{array}{l}\frac{0}{0} \\
\frac{0}{\tilde{J}} \\
\frac{0}{1} \\
0 \\
0\end{array}$} & SCo-52 & 52 & 0.07 \\
\hline & SCo-54 & 54 & 0.015 \\
\hline & SCo-56 & 56 & 0.07 \\
\hline & SCo-58 & 58 & 0.07 \\
\hline \multirow{4}{*}{ 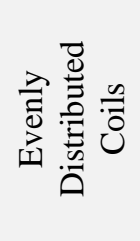 } & SMC-52 & 52 & 0.07 \\
\hline & SMC-54 & 54 & 0.015 \\
\hline & SMC-56 & 56 & 0.07 \\
\hline & SMC-58 & 58 & 0.07 \\
\hline \multirow{4}{*}{ 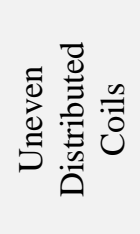 } & SIC-52 & 52 & 0.07 \\
\hline & SIC-54 & 54 & 0.015 \\
\hline & SIC-56 & 56 & 0.07 \\
\hline & SIC-58 & 58 & 0.07 \\
\hline
\end{tabular}

\subsubsection{Experimental Verification}

A schematic depicting the setup of the test model for the experimental setup is illustrated in Figure 4.8 (a), which consists of a shaker, energy harvester, transducers, and data acquisition hardware (DAQ). Figure 4.8 (b) shows a photograph of the experimental setup. The energy harvester device can be seen attached to an electro-dynamic shaker. The harmonic excitation is created using a waveform generator whose output is supplied to an amplifier. The amplified signal drives the actuator onto which the test specimen is mounted. 


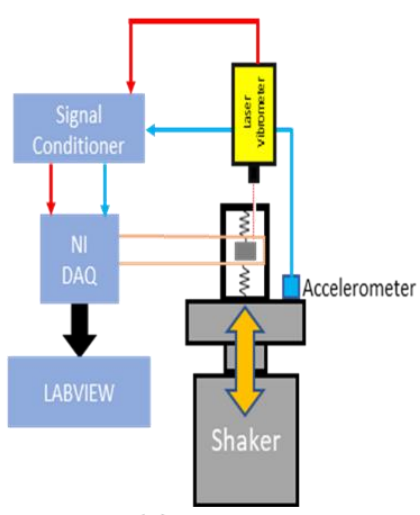

(a)

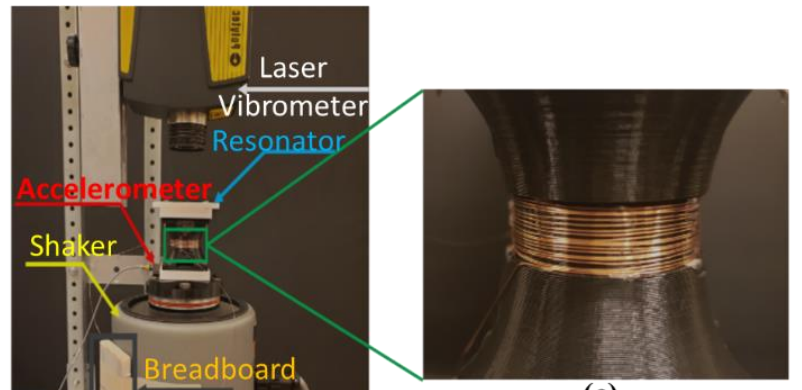

(c)

Figure 4.8: Schematic of (a) experimental model, (b) image from experiment setup, (c) close-up look at the harvesting coils.

The response of the magnet is collected using a laser vibrometer, while an accelerometer measures the input displacement. Furthermore, the voltage generated by the harvesting coils is measured using an NI DAQ. The code can be found in Appendix A.4.

Table 4.4: Summary of test case studies for the proposed experimental model.

\begin{tabular}{|c|c|c|}
\hline \multicolumn{3}{|c|}{ Experimental Model } \\
\hline Test Cases & $\begin{array}{c}\text { Excitation } \\
\text { Frequency } \\
(\mathbf{H z})\end{array}$ & $\begin{array}{c}\text { Input } \\
\text { Displacement } \\
(\mathbf{m m})\end{array}$ \\
\hline E-52 & 52 & 0.07 \\
\hline E-54 & 54 & 0.035 \\
\hline E-56 & 56 & 0.07 \\
\hline E-58 & 58 & 0.07 \\
\hline
\end{tabular}

For the experimental study we only consider the uneven set of coils. The results from theoretical and semi-analytical will be compared against experiment to study which coil configuration predicts the EMF with greater accuracy. 


\subsubsection{Metrics for EMF Study}

The effective voltage of an alternating current can be obtained by applying the root mean square (RMS), given in Equation (4.17). RMS is a fundamental measurement used to find the average magnitude of a varying voltage. It is a mathematical quantity used to compare both alternating and direct currents. For example, a direct current connected to a resistor for a given period would generate the same energy as that produced by the alternating current when passed through the same resistor for the same time. Equations for RMS voltage, power, and percentage error are listed below.

$$
\begin{gathered}
V_{R M S}=\sqrt{\frac{\sum_{i=1}^{n} V_{i}}{n}} \\
\text { Power }=\frac{V_{R M S}{ }^{2}}{\text { Resistor }} \\
\% \text { error }=\frac{\mid \text { Theoretical value }- \text { Exact value } \mid}{\text { Exact value }} \times 100 \%
\end{gathered}
$$

Where $V_{R M S}$ is the root mean square voltage, $V_{i}$ is the voltage for each time interval, and $n$ corresponds to the number of data points. Equation (4.19) will be used as a metric to compare theory to experiment.

\subsection{EMF for Best Assonant Cases}

The AMB partially sequesters the displacement of the incident wave into the resonators, thus providing attenuation in the host structure. The resonators will oscillate about their mean position; this energy may then be converted to electrical energy. In this section, we will be investigating the effect of the energy harvesters within an AMB; the study will be performed for only the best assonant cases (SR14, SQ6, and SM6). Note that from Table 4.3, SQ6 and SM6 have 
similar resonator configurations; however, for SM6, a host material variation with a high degree of impedance mismatch was introduced.

Table 4.5: Summary of resonator configuration for best assonant cases from each case study performed.

\begin{tabular}{c|c|c}
\hline Case No. & Description & Resonator Config. \\
\hline SR14 & AMB - Four resonator-type & $\begin{array}{c}6 \times 100+6 \times 300+5 \times 700+3 \times 1500 \\
\mathrm{~Hz}\end{array}$ \\
\hline SQ6 & $\begin{array}{c}\text { AMB }- \text { Resonator sequence } \\
\text { variation }\end{array}$ & $\begin{array}{c}6 \times 100+5 \times 700+3 \times 1500+6 \times 300 \\
\mathrm{~Hz}\end{array}$ \\
\hline SM6 & $\begin{array}{c}\text { AMB - Host material } \\
\text { stiffness variation }\end{array}$ & $\begin{array}{c}6 \times 100+5 \times 700+3 \times 1500+6 \times 300 \\
\mathrm{~Hz} \text { (Same as SQ6) }\end{array}$ \\
\hline
\end{tabular}

To study the EMF generated for a one-dimensional AMB by assuming a single loop of wire located at the mean position of the resonators of each unit cell, as shown in Figure 4.10, is proposed. The circuit diagram depicts harvester cells connected in series attached to a $1 \Omega$ load resistor. The choice of this resistance value is simply to the aid for comparing the power generation amongst the cases.

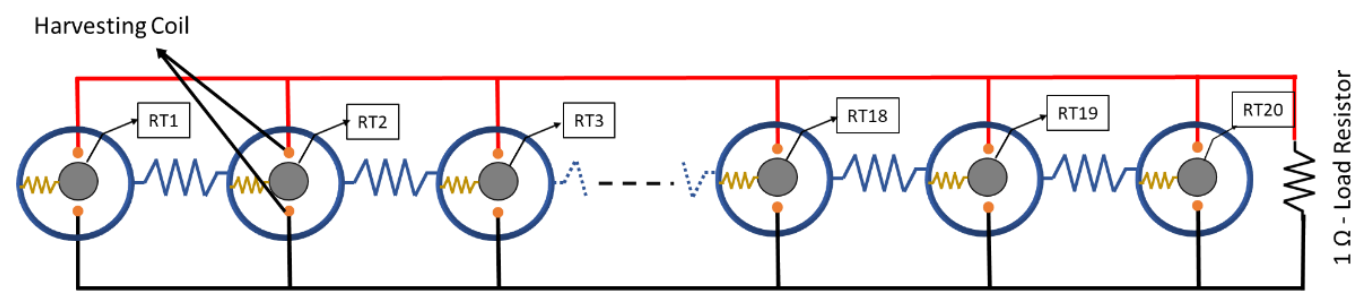

Figure 4.9: A schematic for AM harvester cells connected in series.

To undergo this study, the relative displacements are extracted from the numerical studies and processed into the EMF code along with the given magnet properties in Table 4.4. The relative displacement can easily be estimated by calculating the difference between the response displacement of the resonator $\left(u_{2}^{j}\right)$ and host $\left(u_{1}^{j}\right)$ mass. 


$$
R T^{j}=u_{2}^{j}-u_{1}^{j}
$$

where, $R T^{j}$ represents the relative displacement for each resonator located within the unit cell. The EMF code can be found in Appendix A.7.

Table 4.6: Summary of magnet properties used for the best assonant cases.

\begin{tabular}{|c|c|c|c|c|}
\hline & & & \\
\hline & & Symbols & Values & Units \\
\hline \multirow{3}{*}{ 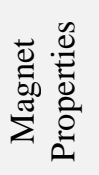 } & Magnet length & $l_{m}$ & 0.0254 & $\mathrm{~m}$ \\
\hline & Magnet radius & $R_{m}$ & 0.0254 & $\mathrm{~m}$ \\
\hline & Magnet dipole moment & $m_{d}$ & 60.6 & A. $m^{2}$ \\
\hline
\end{tabular}

\subsection{EMF Rectification}

The two conventional devices to store electrical energy for multiple use are a rechargeable battery and capacitors. Since the direction of current changes in an alternating voltage source, the harvested energy cannot be stored directly. However, to store this energy, the alternating current can be converted to direct current using rectifiers. In this section, a theoretical comparison study between sum-rectify and rectify-sum is defined. Sum-rectify refers to rectifying the total harvested voltage. On the contrary, rectify-sum will convert each harvested AC electricity to DC within every unit cell and adds the values to get the total voltage within the AMB. A full-wave rectifier flips the negative side of an alternating current source to a positive, and this is achieved by using rectifiers in a practical scenario. However, for our study, we will only be doing a theoretical rectification, and that can be achieved simply by taking the absolute value of the voltage signal in the time domain.

\subsection{Discussion of Results}

The results for magnetic field plots, EMF verification, best EMF assonant mechanisms, and rectification will be discussed in this section. 


\subsubsection{Magnetic Field Plots}

Figure 4.10 and 4.11 depicts the surface plot magnetic field lines in the axial and radial directions, respectively. Due to cylindrical symmetry assumptions, B-field is a mirror of itself about the axial and radial directions.

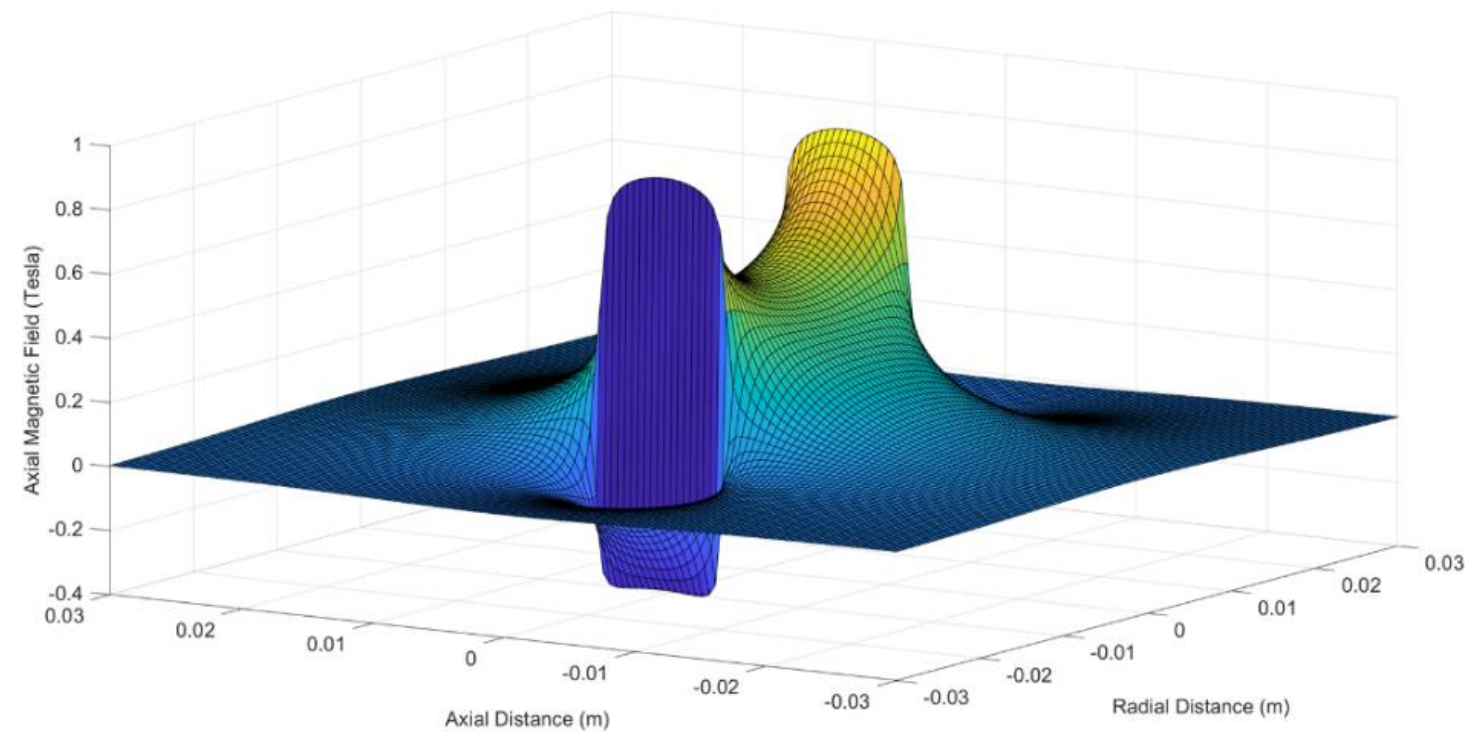

Figure 4.10: Surface plot of the magnetic field in the axial direction.

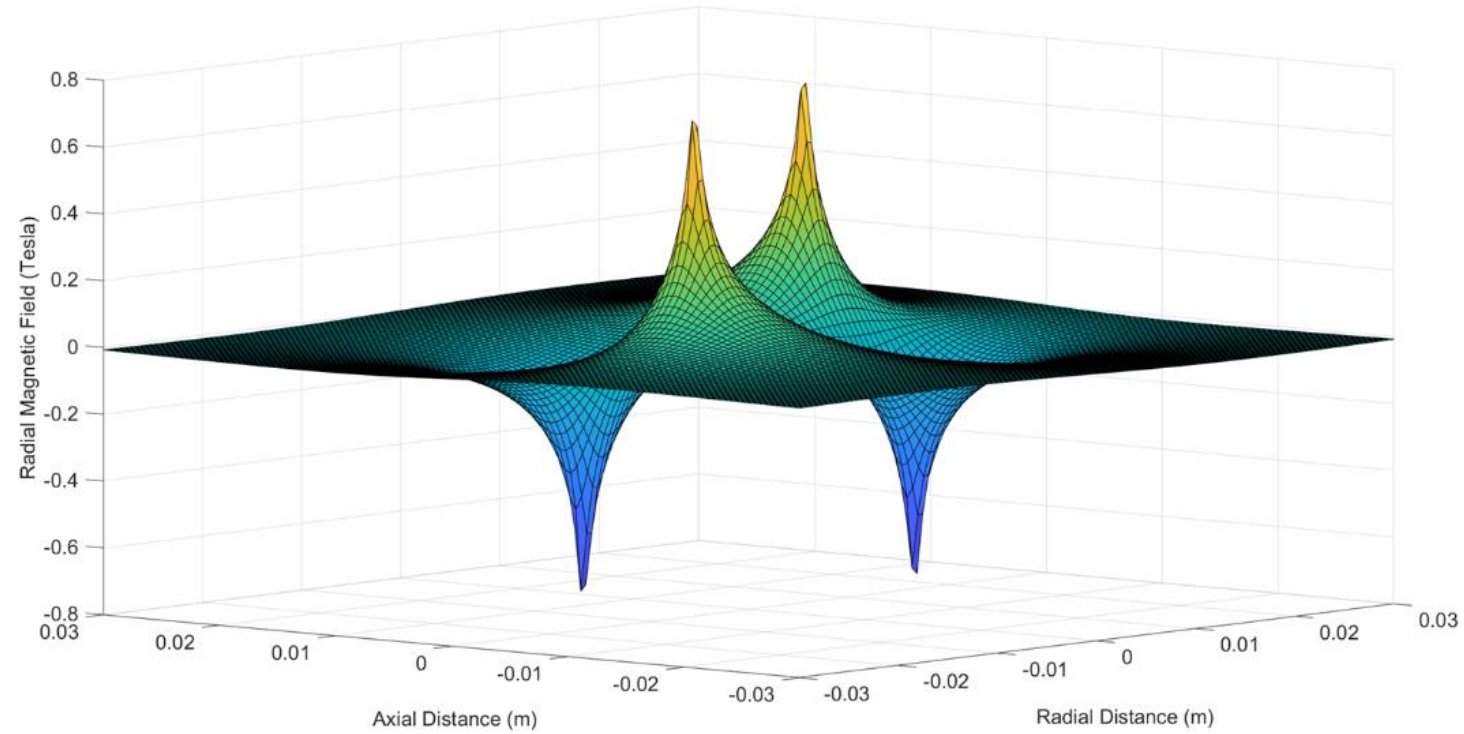

Figure 4.11: Surface plot of the magnetic field in the radial direction. 
The axial field lines are maximum at the origin, $\rho=0$ and $z=0$. The discontinuity seen in Figure 4.11 occurs when the magnetic field lines change directions along the axial axis at ( $\rho= \pm a$ and $z= \pm l / 2)$. The magnetic field in the radial direction is zero at the origin and maximum when $\rho= \pm a$ and $z= \pm l / 2$.

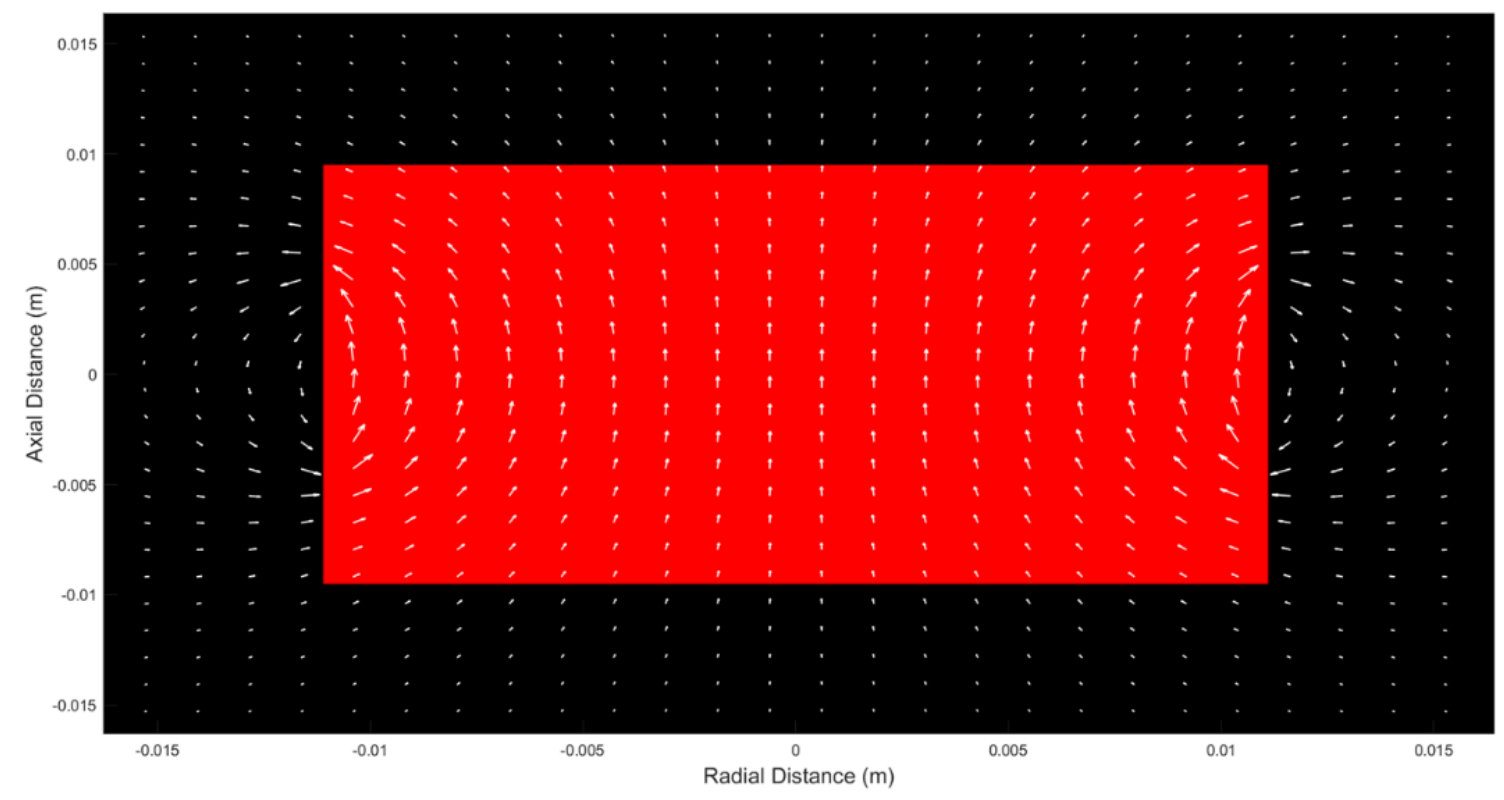

Figure 4.12: Magnetic field lines of a Neodymium magnet.

Figure 4.12 shows the mapping of the magnetic field in the radial and axial direction simultaneously. The axial field lines point straight up close to the center of the magnet. As the radial direction is increased from the origin, then the radial influence can be observed and thus starts to bend the magnet towards the south pole. Magnetic field lines may be used as a qualitative tool to visualize magnetic forces. 


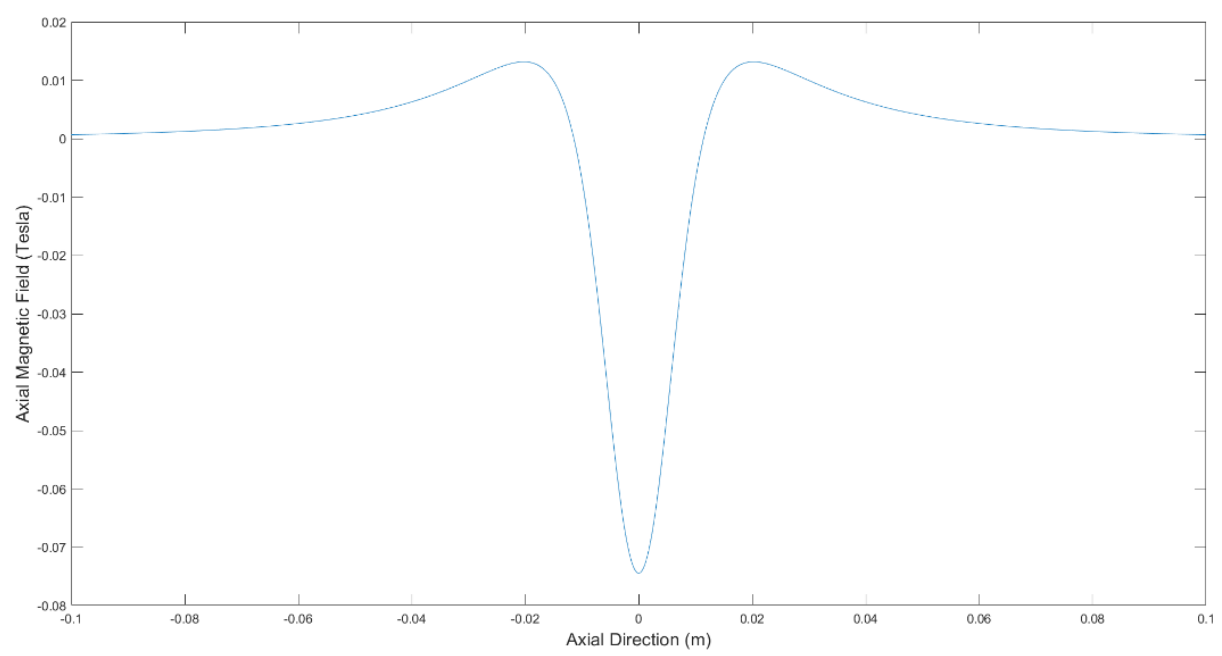

Figure 4.13: Axial magnetic field plot at $\rho=19 \mathrm{~mm}$.

Figure 4.13 depicts the axial magnetic field at $\rho=19 \mathrm{~mm}$ from the magnet's center. Note that when approaching the origin along the axial direction, the slope of the magnetic field tends to zero. Recall, Equation (4.3) where the product of two slopes, (i) velocity versus time data, $\frac{\mathrm{d} z}{\mathrm{~d} t}$ and (ii) magnetic field versus position, $\frac{\mathrm{d} B_{z}}{\mathrm{~d} z}$. One can observe from Figure 4.14 that the slope of the magnetic field is zero when approaching the origin, and this tells us that for a magnet moving with a small amplitude, the generated EMF may complete two cycles for half a revolution of the velocity. From this, one can expect the EMF to double its harvested frequency. The verification of this observation will be discussed in a later section.

\subsubsection{Translational EMF Verification}

The EMF verification for all categories of coil placement will be discussed below. We will discuss the relevant EMF results for experiments carried out. The displacement response time history and frequency spectra charts could be found in the appendix sections. 


\subsubsection{EMF Results for Co-located Configuration}

This section discusses the results for the co-located coil assumption given in Equation (4.5). The co-located assumption neglects the axial separation created by the thickness of the coils and assumes that all the wires exhibit the same magnetic field change. As per the defined test cases in Table 4.2, the results for the co-located coil configuration are shown in the figures below. The results for $56 \mathrm{~Hz}$ and $58 \mathrm{~Hz}$ can be found in Appendix B.1.

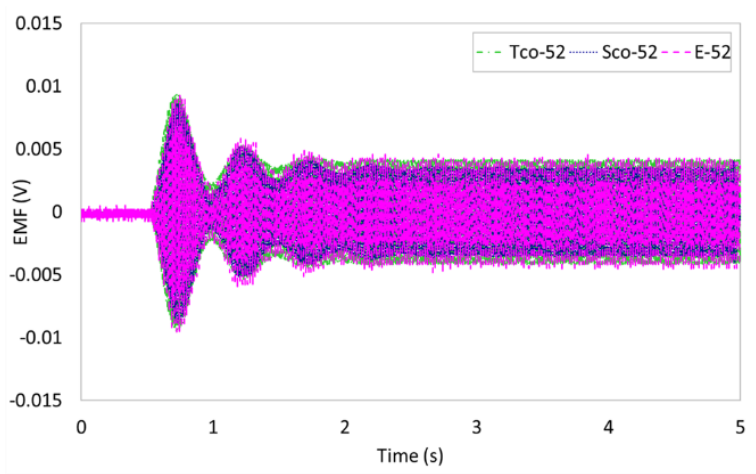

(a)

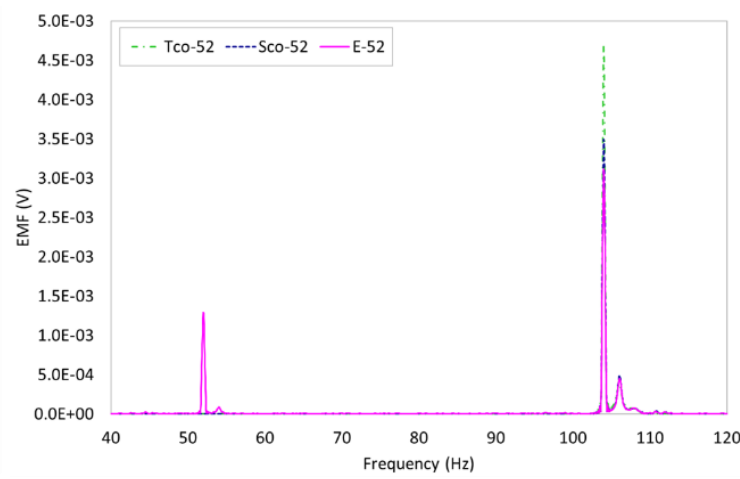

(b)

Figure 4.14: EMF data, (a) time history and (b) frequency spectra plots for $52 \mathrm{~Hz}$ excitation input with co-located coil configuration

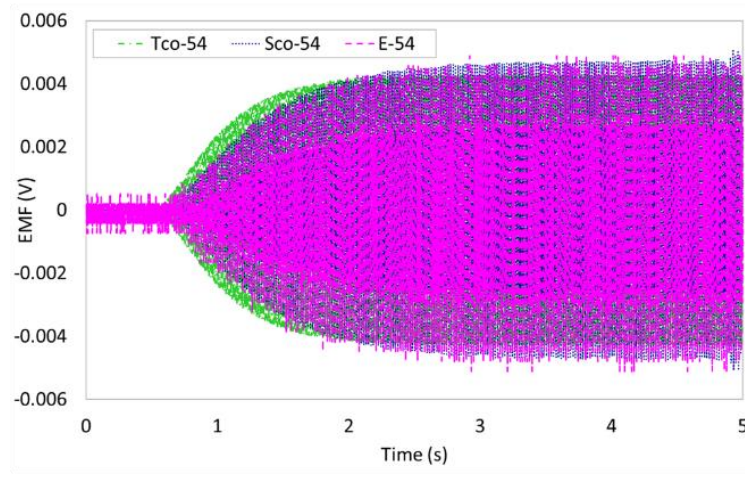

(a)

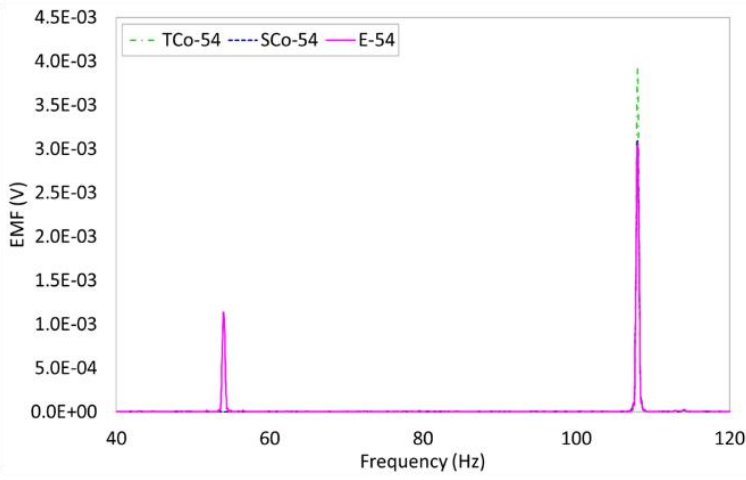

(b)

Figure 4.15: EMF data, (a) time history and (b) frequency spectra plots for $54 \mathrm{~Hz}$ excitation input with co-located coil configuration 
By observing the time history charts for the theoretical and semi-analytical model, it can be said that the EMF results are predicting within a reasonable range. In addition, the root mean square voltage for the defined cases summarized in Table 4.5 proves to show best prediction for semi-analytical co-located coil. However, the frequency spectrum figure for the experimental result depicts two frequency peaks at the excitation frequency and its double.

Table 4.7: Summary of $V_{r m s}$ and percentage error data for theoretical (TCo) and semi-analytical (SCo) co-located coil configuration

\begin{tabular}{c|c|c}
\hline Test Cases & $\mathrm{V}_{r m s}(\mathrm{~V})$ & $\begin{array}{c}\text { Percentage } \\
\text { error }(\%)\end{array}$ \\
\hline TCo-52 & 3 & 19.4 \\
\hline TCo-54 & 2.6 & 17.4 \\
\hline TCo-56 & 3.5 & 9.4 \\
\hline TCo-58 & 0.5 & 40.5 \\
\hline SCo-52 & 2.6 & 3.1 \\
\hline SCo-54 & 2.1 & 5.73 \\
\hline SCo-56 & 3.3 & 3.1 \\
\hline SCo-58 & 0.7 & 16.7 \\
\hline
\end{tabular}

The co-located coils for both theoretical and semi-analytical cases do not pick up the harvested voltage that coincides with the excitation frequency. The fact that the coils are assumed to be located at the origin, the magnetic field experienced by each coil remains the same and that the field polarity relative to the coils changes twice in one cycle. From the displacement and EMF plots a beating effect during start up transients can be observed for all excitation frequencies except at the natural frequency. 


\subsubsection{EMF Results for Mirror Configuration}

The mirror coil configuration assumption takes in consideration that the two set of harvesting wires are symmetrical to one another as depicted in Figure 4.4 (b). The displacement response plots can be found in Appendix B.4 - B.7.

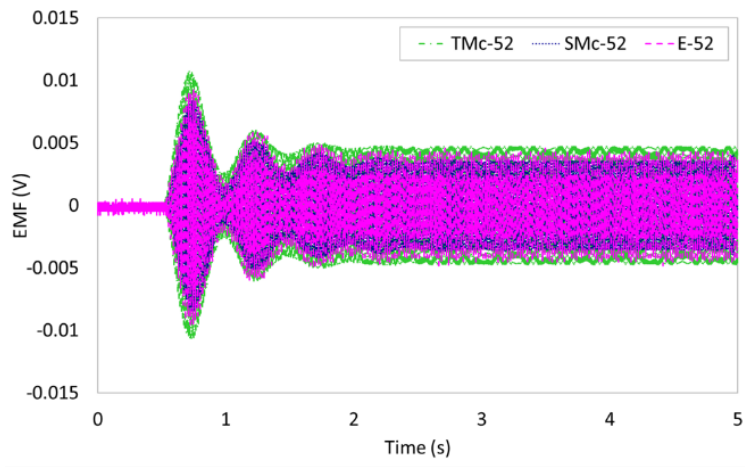

(a)

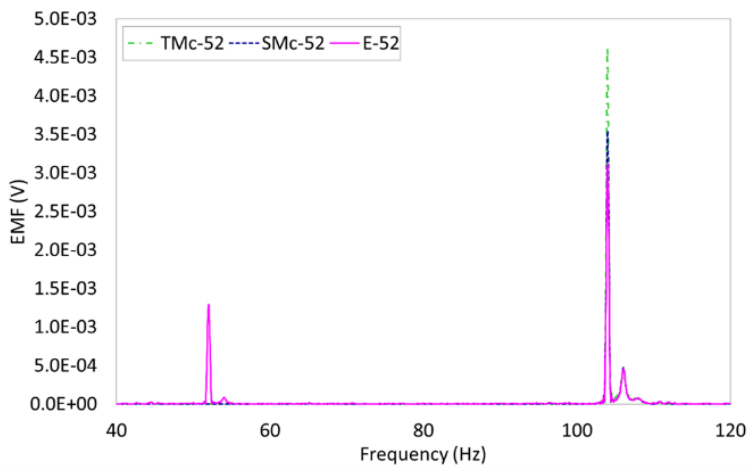

(b)

Figure 4.16: EMF data, (a) time history and (b) frequency spectra plots for $52 \mathrm{~Hz}$ excitation

$$
\text { input with mirror coil configuration }
$$

In relation to the results observed in the co-located configuration, the harvested voltage signal peaks only at a frequency twice of the base excitation frequency. Even though the coils are assumed to be distributed, the mirroring configuration assumes that each harvesting wire are equidistant above and below the origin thus effectively experiencing equal and opposite magnetic field.
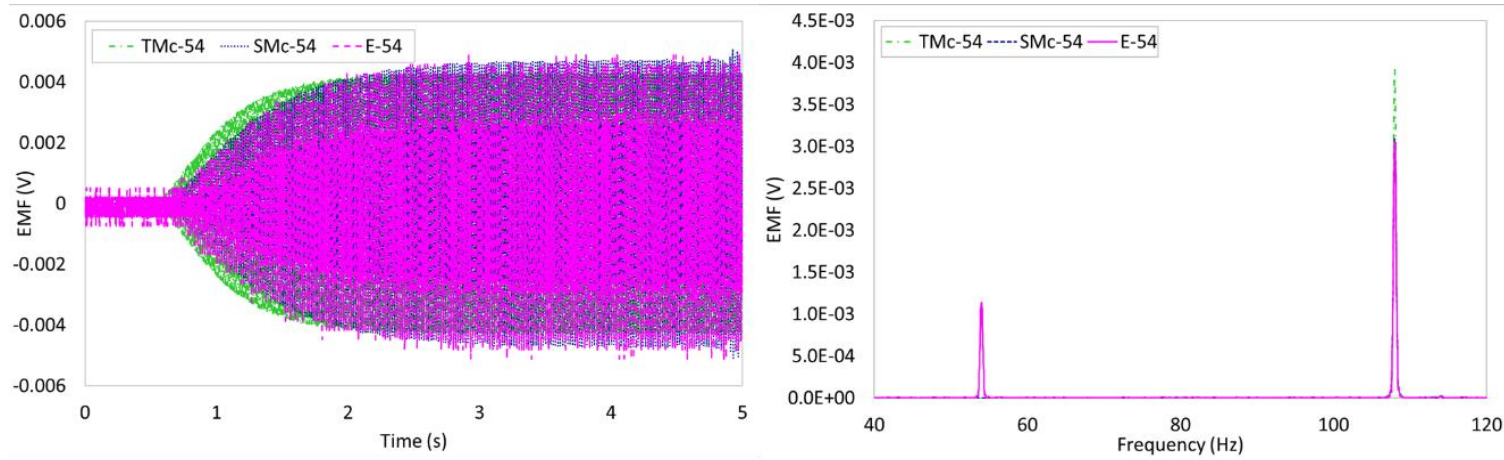

Figure 4.17: EMF data, (a) time history and (b) frequency spectra plots for $54 \mathrm{~Hz}$ excitation input with mirror coil configuration 
To mitigate this, one can rectify each set of coils or use a phase synchronizer. Figure 4.16 and 4.17 depicts the EMF time history and frequency spectra charts for $52 \mathrm{~Hz}$ and $54 \mathrm{~Hz}$, respectively. The EMF plots for $56 \mathrm{~Hz}$ and $58 \mathrm{~Hz}$ can be found in Appendix B.2.

Table 4.8: Summary of $V_{r m s}$ and percentage error data for theoretical (TMC) and semianalytical (SMC) mirror coil configuration

\begin{tabular}{c|c|c}
\hline Test Cases & $\mathrm{V}_{r m s}(\mathrm{~V})$ & $\begin{array}{c}\text { Percentage } \\
\text { error }(\%)\end{array}$ \\
\hline TMC-52 & 2.9 & 17.02 \\
\hline TMC-54 & 2.6 & 16.05 \\
\hline TMC-56 & 3.3 & 3.1 \\
\hline TMC-58 & 0.5 & 40.5 \\
\hline SMC-52 & 2.6 & 3.71 \\
\hline SMC-54 & 2.1 & 5.61 \\
\hline SMC-56 & 3.3 & 3.1 \\
\hline SMC-58 & 0.95 & 13.1 \\
\hline
\end{tabular}

\subsubsection{EMF Results for Interspersed Configuration}

The actual arrangement of coils that were setup to conduct the experiment were positioned randomly along the vertical axis as depicted in Figure 4.4 (c) and 4.8 (c). The time histories and frequency spectrum plots can be seen in Figure 4.18 and Figure 4.19. The RMS voltage values for theoretical and semi-analytical studies, proof that the taking the summation of the harvested voltage for each coil provides an accurate description of generated EMF. In addition, the frequency spectra plots for this coil configuration shows that both $52 \mathrm{~Hz}$ and $104 \mathrm{~Hz}$ component are predicted with greater accuracy. 


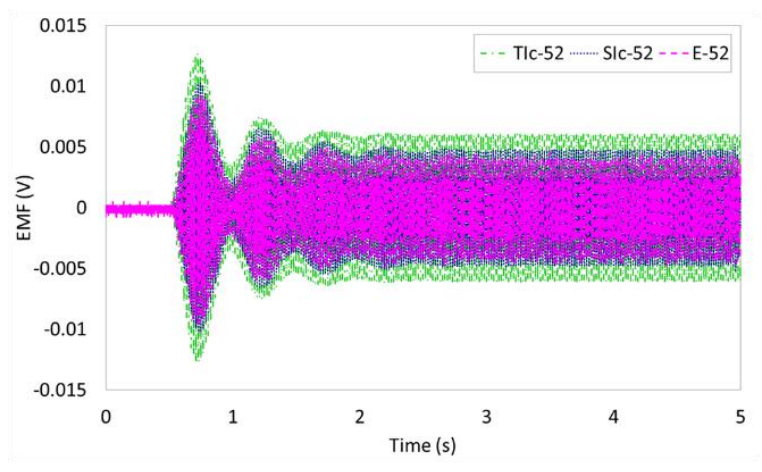

(a)

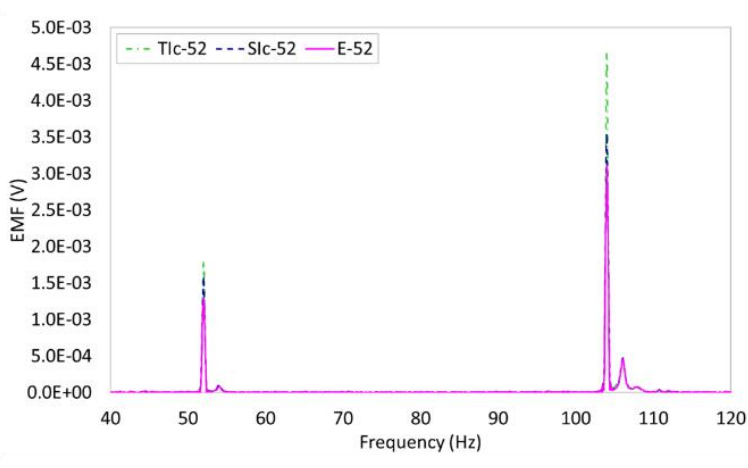

(b)

Figure 4.18: EMF data, (a) time history and (b) frequency spectra plots for $52 \mathrm{~Hz}$ excitation input with interspersed coil configuration

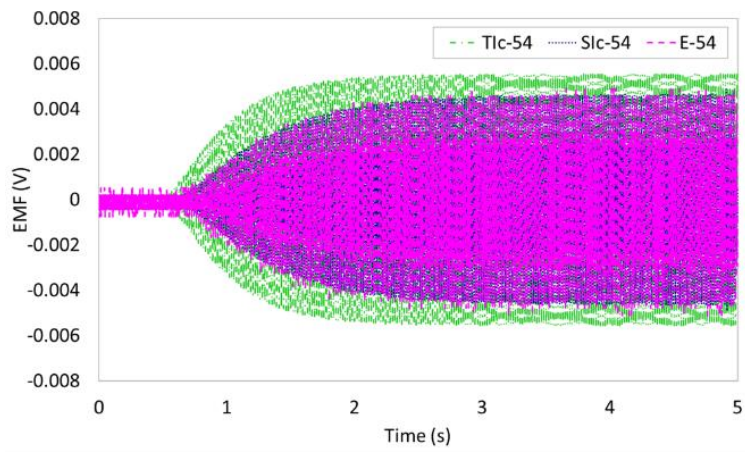

(a)

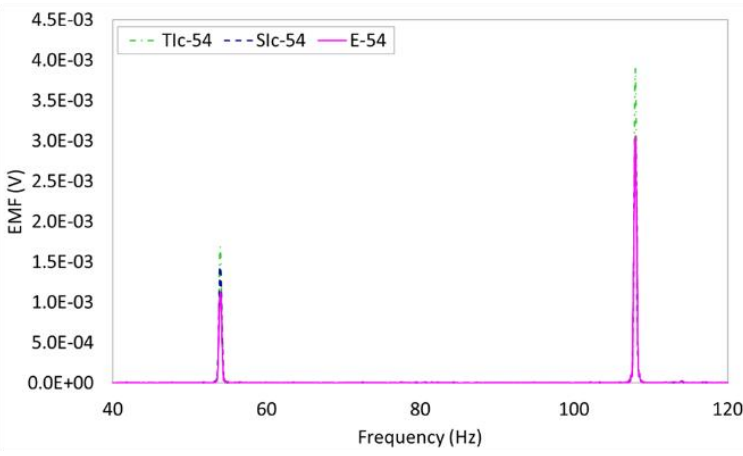

(b)

Figure 4.19: EMF data, (a) time history and (b) frequency spectra plots for $54 \mathrm{~Hz}$ excitation input with interspersed coil configuration

In general, from these sets of EMF studies we have verified the algorithm and equation constructed for the EMF code. Furthermore, to improve the accuracy of the code an algorithm was developed to calculate the EMF results for each coil which then takes the summation to get the total harvested voltage. The EMF code and the functions associated with it can be found in Appendix A.7. 
Table 4.9: Summary of $V_{r m s}$ and percentage error data for theoretical (TIC) and semi-analytical (SIC) interspersed coil configuration

\begin{tabular}{c|c|c}
\hline $\begin{array}{c}\text { Test } \\
\text { Cases }\end{array}$ & $\mathrm{V}_{r m s}(\mathrm{~V})$ & $\begin{array}{c}\text { Percentage } \\
\text { error }(\%)\end{array}$ \\
\hline TIC-52 & 2.8 & 13 \\
\hline TIC-54 & 2.5 & 12.11 \\
\hline TIC-56 & 3.5 & 9.4 \\
\hline TIC-58 & 0.61 & 27.4 \\
\hline SIC-52 & 2.6 & 2.5 \\
\hline SIC-54 & 2.2 & 0.91 \\
\hline SIC-56 & 3.3 & 3.1 \\
\hline SIC-58 & 0.82 & 2.4 \\
\hline
\end{tabular}

\subsubsection{EMF for Best Assonant Cases}

This section discusses the EMF results for the best resonator-type, resonator sequence, and material variation cases, which are summarized in Table 4.3. The time history and frequency spectrum for harvested EMF will be addressed for all twenty resonators within the AMB. Furthermore, the results for sum-rectify and rectify-sum will be discussed for each case.

\subsubsection{Resonator-Type Variation Case}

The harvested EMF from each resonator for the best assonant mechanism in the resonatortype variation case, SR14, will be discussed. The displacement time histories and FFT results are addressed for all sets of resonators in Appendix B.8.1 - B.8.4. Furthermore, EMF time histories and FFT results are illustrated in Appendix B.8.5 - B.8.8. Table 4.8 summarizes harvested voltage for each resonator. The relative displacement of each unit cell can be seen increasing across the AMB except for the $1500 \mathrm{~Hz}$ set of resonators. This behavior is also true for the harvested EMF. 
The $1500 \mathrm{~Hz}$ component of the incident pulse has a displacement, which is almost half of the 700 $\mathrm{Hz}, 300 \mathrm{~Hz}$, and $100 \mathrm{~Hz}$; therefore, the available energy is much less than the other resonators.

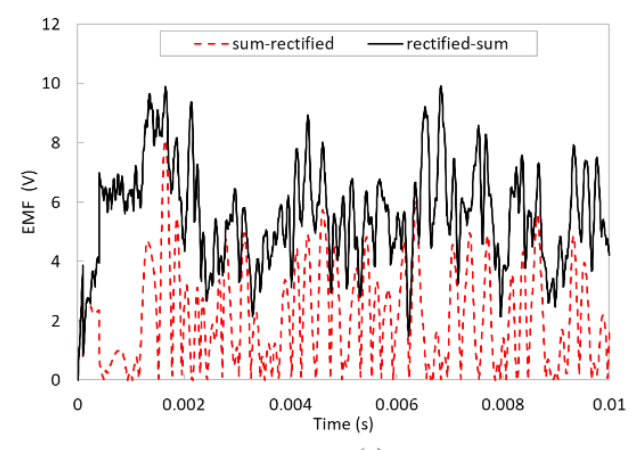

(a)

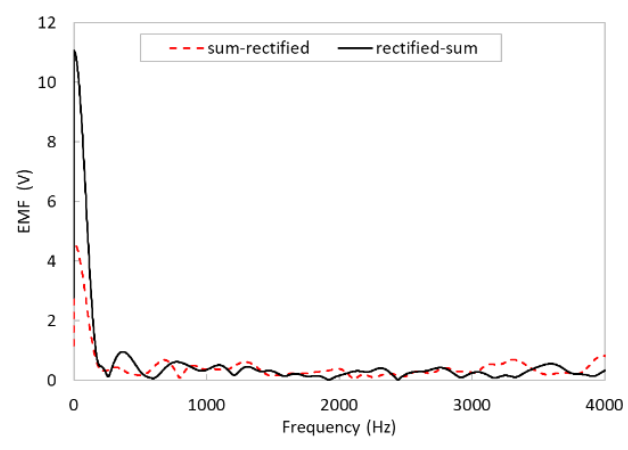

(b)

Figure 4.20: Rectified EMF (a) time history and (b) frequency spectrum plot for best resonatortype variation case (SR14).

Table 4.10: Summary of harvested voltage RMS for best resonator-type variation case (SR14).

\begin{tabular}{|c|c|c|c|}
\hline $\begin{array}{c}\text { Resonator } \\
\text { sets }\end{array}$ & Resonator & $V_{R M S}(\mathrm{~V})$ & $\begin{array}{l}V_{R M S} \text { for each set } \\
\text { of resonators }(\mathrm{V})\end{array}$ \\
\hline \multirow{6}{*}{ 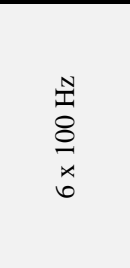 } & RT1 & 0.3 & \multirow{6}{*}{2.4} \\
\hline & RT2 & 0.3 & \\
\hline & RT3 & 0.4 & \\
\hline & RT4 & 0.5 & \\
\hline & RT5 & 0.4 & \\
\hline & RT6 & 0.5 & \\
\hline \multirow{6}{*}{ 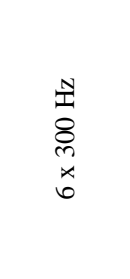 } & RT7 & 0.5 & \multirow{6}{*}{2.7} \\
\hline & RT8 & 0.5 & \\
\hline & RT9 & 0.4 & \\
\hline & RT10 & 0.4 & \\
\hline & RT11 & 0.5 & \\
\hline & RT12 & 0.4 & \\
\hline \multirow{5}{*}{\begin{tabular}{l} 
N \\
\multirow{2}{*}{} \\
8 \\
$x$ \\
$n$ \\
$n$
\end{tabular}} & RT13 & 0.8 & \multirow{5}{*}{3.0} \\
\hline & RT14 & 0.7 & \\
\hline & RT15 & 0.6 & \\
\hline & RT16 & 0.5 & \\
\hline & RT17 & 0.4 & \\
\hline \multirow{3}{*}{$\underset{\substack{\infty\\
}}{\stackrel{8}{2}}$} & RT18 & 0.4 & \multirow{3}{*}{1.0} \\
\hline & RT19 & 0.3 & \\
\hline & RT20 & 0.3 & \\
\hline
\end{tabular}


The dominant frequency content of the relative displacement ranges from about 100-2000 $\mathrm{Hz}$; however, this can be seen doubled for the generated EMF ranging from (200-4000 Hz). The total theoretical harvested power for SR14 is calculated to be 82.8 Watt on across a $1 \Omega$ load resistor. Rectified signals for SR14 can be observed in Figure 4.20. sum-rectify yields about 60\% less harvested voltage than rectifying rectify-sum. The phase difference of the resonators creates a destructive interference when the summation of the signal is taken without initially rectifying. Also, rectify-sum generates $11 \mathrm{~V}$, which is in a reasonable range to the calculated root mean square voltage of $9.2 \mathrm{~V}$. In practice rectifying each unit cell might be difficult; therefore, to manufacture such a harvesting device might become costly.

\subsubsection{Best Resonator Sequence Variation Case (SQ6)}

For the best resonator sequence variation (SQ6) case, the relative position of resonators within the best performing SR case (SR14) are varied by moving the $300 \mathrm{~Hz}$ set resonators to take the last six position in the $\mathrm{AMB}$ while the $100 \mathrm{~Hz}$ resonator retains its position. The charts for the displacement and EMF time history and frequency spectra can be found in Appendix B.9.1 -B.9.8. There is a significant decrease in relative displacement and harvested EMF for the $300 \mathrm{~Hz}$ resonator as it is moved further away from the incident side, while the $100 \mathrm{~Hz}$ resonator remains about the same compared to that seen in SR14. Also, the $700 \mathrm{~Hz}$ resonators generate the highest voltage in this case which is consistent with the previous case.

Like SR14 the rectify-sum yields the best harvested DC voltage. Sum-rectify harvests EMF $60 \%$ lower than rectify-sum as shown in Figure 4.22. The total power harvested for SQ6 is $79.2 \mathrm{~W}$ which is found to be less to that of SR14. This is mainly due to moving the $300 \mathrm{~Hz}$ resonators further away from the incident side. 


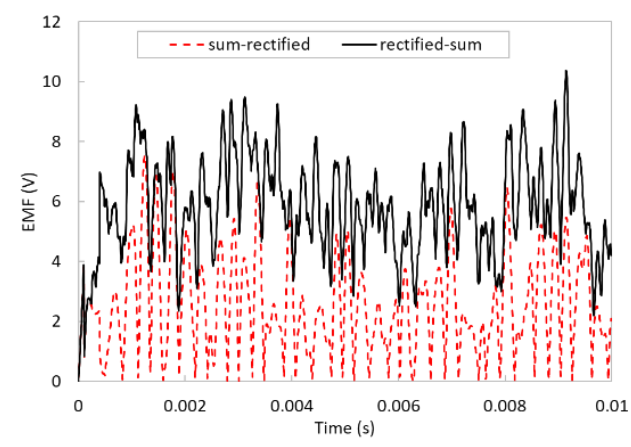

(a)

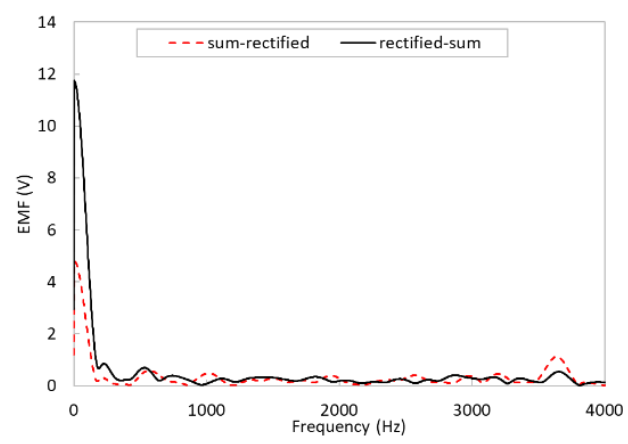

(b)

Figure 4.21: Rectified EMF (a) time history and (b) frequency spectrum plot for best resonator sequence case (SQ6).

Table 4.11: Summary of harvested voltage RMS data for best resonator sequence case (SQ6).

\begin{tabular}{|c|c|c|c|}
\hline $\begin{array}{c}\text { Resonator } \\
\text { sets }\end{array}$ & Resonator & $V_{R M S}(\mathrm{~V})$ & $\begin{array}{l}V_{R M S} \text { for each set } \\
\text { of resonators }(\mathrm{V})\end{array}$ \\
\hline \multirow{6}{*}{ 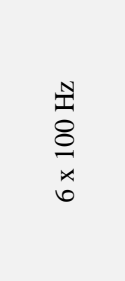 } & RT1 & 0.3 & \multirow{6}{*}{2.9} \\
\hline & RT2 & 0.4 & \\
\hline & RT3 & 0.5 & \\
\hline & RT4 & 0.6 & \\
\hline & RT5 & 0.5 & \\
\hline & RT6 & 0.6 & \\
\hline \multirow{5}{*}{$\begin{array}{l}\stackrel{y}{1} \\
8 \\
8 \\
x \\
x \\
n\end{array}$} & RT7 & 1.1 & \multirow{5}{*}{3.3} \\
\hline & RT8 & 0.8 & \\
\hline & RT9 & 0.6 & \\
\hline & RT10 & 0.5 & \\
\hline & RT11 & 0.3 & \\
\hline \multirow{3}{*}{ 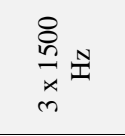 } & RT12 & 0.3 & \multirow{3}{*}{1} \\
\hline & RT13 & 0.5 & \\
\hline & RT14 & 0.2 & \\
\hline \multirow{6}{*}{ 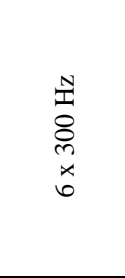 } & RT15 & 0.3 & \multirow{6}{*}{1.7} \\
\hline & RT16 & 0.3 & \\
\hline & RT17 & 0.3 & \\
\hline & RT18 & 0.2 & \\
\hline & RT19 & 0.3 & \\
\hline & RT20 & 0.3 & \\
\hline \multicolumn{4}{|c|}{ Total $V_{R M S}=8.9 \mathrm{~V}$} \\
\hline
\end{tabular}




\subsubsection{Best Material Variation Case (SM6)}

The best host material variation case is found to be SM6 in which a unit with 'soft' host material stiffness is sandwiched between two sets of identical 'hard' host material. This configuration induces a high degree of impedance mismatch. Figure B.10.1 - B.10.4 illustrates the relative displacements for all resonators. The resonators placed after the 'soft' material has significant decrease in displacement whereas the preceding resonators experience a large displacement. This shows that most of the wave is attenuated within the first 'hard' material region because of the high impedance mismatch.

Table 4.12: Summary of harvested voltage RMS data for best material variation case (SM6).

\begin{tabular}{|c|c|c|c|}
\hline $\begin{array}{l}\text { Resonator } \\
\text { sets }\end{array}$ & Resonator & $V_{R M S}(\mathrm{~V})$ & $\begin{array}{l}V_{R M S} \text { for each set } \\
\text { of resonators }(\mathrm{V})\end{array}$ \\
\hline \multirow{6}{*}{ 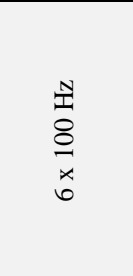 } & RT1 & 0.3 & \multirow{6}{*}{3.3} \\
\hline & RT2 & 0.4 & \\
\hline & RT3 & 0.4 & \\
\hline & RT4 & 0.6 & \\
\hline & RT5 & 0.7 & \\
\hline & RT6 & 0.9 & \\
\hline \multirow{5}{*}{$\begin{array}{l}\underset{1}{1} \\
8 \\
8 \\
\dot{8} \\
x \\
n\end{array}$} & RT7 & 3.5 & \multirow{5}{*}{14.1} \\
\hline & RT8 & 3.8 & \\
\hline & RT9 & 4.0 & \\
\hline & RT10 & 2.5 & \\
\hline & RT11 & 0.3 & \\
\hline \multirow{3}{*}{ 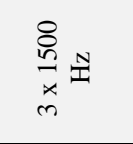 } & RT12 & $2.6 \mathrm{E}-4$ & \multirow{3}{*}{$7.8 \mathrm{E}-4$} \\
\hline & RT13 & $2.6 \mathrm{E}-4$ & \\
\hline & RT14 & $2.6 \mathrm{E}-4$ & \\
\hline \multirow{6}{*}{ 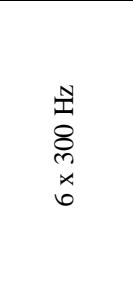 } & RT15 & 0.01 & \multirow{6}{*}{0.06} \\
\hline & RT16 & 0.01 & \\
\hline & RT17 & 0.01 & \\
\hline & RT18 & 0.01 & \\
\hline & RT19 & 0.01 & \\
\hline & RT20 & 0.01 & \\
\hline
\end{tabular}


B.10.5 - B.10.8 shows the harvested EMF time histories and frequency spectra. The 700 $\mathrm{Hz}$ and $100 \mathrm{~Hz}$ resonators account for much of the harvested EMF within the AMB. The total harvested voltage is $17.5 \mathrm{~V}$ which is almost double of the harvested voltage by both SR14 and SQ6, as shown in Table 4.10; the total harvested power for SM6 is 306.3 Watt. By observing Figure 4.22, the sum-rectify and rectify-sum generate about the same voltage; this also suggests that the resonators are in-phase with one another this behavior is due to the high degree impedance mismatch.

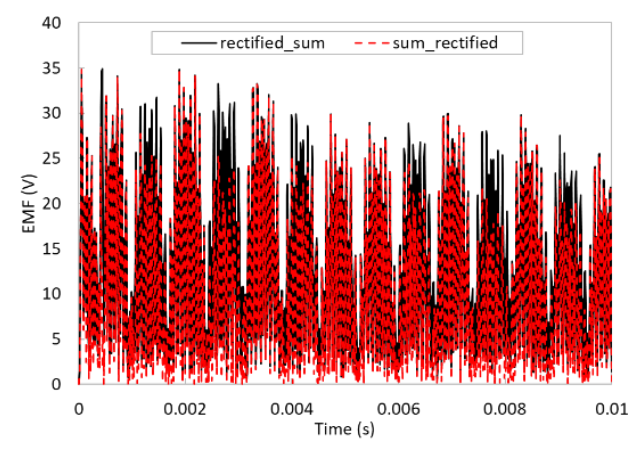

(a)

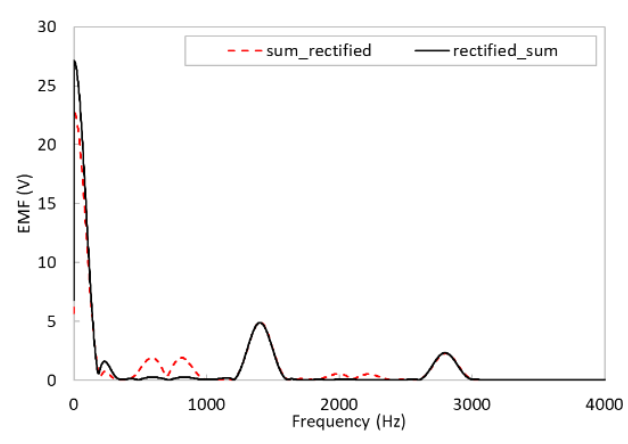

(b)

Figure 4.22: Rectified EMF (a) time history and (b) frequency spectrum plot for best material variation case (SM6).

\section{$\underline{4.8 \text { Summary }}$}

In this Chapter, the investigation of linear electromechanical energy harvesting application for mechanically assonant acoustic metamaterial was demonstrated. The derivation and code to calculate translational EMF and magnetic field was verified by comparing theoretical to experimental studies. Some notable results that were found are:

- The coil configuration and position relative to the location of the magnet is seen to affect the frequency of the harvested voltage. 
- For co-located and mirror coil configuration, the harvested voltage's frequency is seen to be at double the excitation frequency. This is expected as the magnetic field around the coils change twice in just half a cycle of the magnet's displacement.

- In the case of mirror coil configuration, the $52 \mathrm{~Hz}$ component harvested by the pair of coils positioned above and below the origin are $90^{\circ}$ out of phase, thus canceling each other out. It may be possible to avoid this by either using phase adjustment or rectification.

- Overall, the semi-analytical model estimates the EMF with less percentage error than the proposed theoretical model. Thus, verifying the assumptions considered in section 4.4.1.1 and 4.4.1.2. The theoretical model assumes damping value, spring stiffness and mass which affects the accuracy of the displacement response of the magnet. Whereas for the case of semi-analytical the magnet's response is measured using a laser vibrometer during the experiment.

- It can be concluded that the best test case for EMF prediction (SIC-58) estimates the harvested signal within $2.4 \%$ error from the actual data. Moreover, the semianalytical interspersed configuration (SIC) predicts the generated voltage with $(<10 \%)$ error.

- Mirror and interspersed coil configuration studies, which use Equation (4.6) yield better prediction of EMF than the co-located assumption given in Equations (4.5). Equation (4.6) estimates the total generated voltage by summing the induced voltage for each coil, whereas Equation (4.5) assumes that all coils are co-located and can be multiplied by $\left(N_{\text {coils }}\right)$ to yield total EMF.

Energy harvesting for a one-dimensional AMB by assuming a single loop of wire positioned at the center of each unit was proposed and studied. It is found that: 
- The harvested EMF for each unit cell is shown to decrease across the AMB, which is contributed by the wave attenuation from the preceding resonators.

- The best performance for harvested voltage is yielded by the material variation case (SM6). It is seen that majority of the harvested voltage in the first 'hard' material region, then a significant decrease in voltage can be seen after the soft material which is mainly due to the high degree of impedance mismatch.

- From the perspective of sequencing and resonator variation (SQ6 and SR14) the harvested voltages results seem close to one another. Thus suggesting, sequencing doesn't have major influence in the performance of the harvester.

- The rectification of an AC voltage to DC was studied by comparing two ideal methods which were, adding all the AC voltages from each unit cell then rectifying (defined as sum-rectify), and rectifying each voltage harvested per unit cell then taking the summation to get the total DC (defined as rectify-sum). This study showed that rectifying prior to connecting each wire gives the higher output in DC voltage.

- For SR14 and SQ6 over 50\% decrease in DC voltage can be seen for the case of sum-rectify compared to rectify-sum technique. For SM6 the rectified signal for both defined techniques lie within less than $10 \%$ of each other as the phase difference between each resonator is seen to be very low. 


\section{CHAPTER V}

\section{CONCLUSION}

\section{$\underline{5.1 \text { Conclusions }}$}

Acoustic metamaterials exhibit unusual properties like negative mass density or negative modulus; these characteristics are not found in natural materials. Thus, giving AM the ability to manipulate waves in any elastic media. An exploratory study was made for a dual-functional mechanically assonant acoustic metamaterial for wave manipulation and energy harvesting. Assonance is a device used in poetry and prose to convey internal rhythm in sounds of words/syllables either between vowels or consonants. A literary analogy was postulated, referencing the local resonators as a vowel, which in English literature is defined as the nucleus of a syllable. In contrast, the host material is referred to as a consonant.

A numerical exploration study was proposed and analyzed for the term 'mechanical assonance', which makes use of interactive mechanisms between the local resonators and host material. Furthermore, a two-parameter study is undertaken for an acoustic metamaterial barrier subjected to broadband disturbance. The purpose of this study is to investigate attenuation performance by mixing various single type-resonators across the AMB with different types of alternating arrangement of external stiffness configuration. Transmissibility metrics are defined to gauge attenuation performance by comparing a baseline (elastic waveguide without resonators) to all $\mathrm{AMB}$ cases. It is important to note that the static stiffness and static mass are kept fixed for all case studies. Based on the result of the study made in Chapter 2 the following conclusion were made: 
- It is found that very low resonator frequencies $\left(<1 / 10^{\text {th }}\right.$ of max bandwidth) are not preferable.

- Subsequent regions with enhanced $\mathrm{D}^{*}$ metrics are found to be spaced in an octave like manner.

- Simultaneously, it is found that when the ratio of the alternating stiffnesses approaches unity the attenuation performance of the AMB is reduced.

- In agreement with the analogy inspired by the literary device assonance, it can be noted that the transmissibility performance of the AMB is predominately influenced by the variation of local resonators (vowels), rather than the alternating arrangement of external stiffness (consonants).

In Chapter 3, a numerical exploration for an acoustic metamaterial barrier (AMB) subjected to a broadband input is carried out to discover relative mechanical wave mitigation performance based on transmissibility metrics. As a measure of equivalence between the baseline parameter and the various AMB cases considered in this study, the static mass and stiffness is retained invariant for all cases. Based on the results of this study, the following conclusions are made:

- The attenuation performance of the AMB can be improved by choosing the number of resonators for each set to be proportional to the amplitude corresponding to its frequency in the input (step) displacement spectrum.

- Simultaneously, it is also found that using an octave spacing for the specific frequencies chosen for resonator sets yielded the best result among the resonator frequency variation cases considered. Amongst the cases considered in this study, the four resonator-type cases outperformed both single resonator-type cases and the $N$ distinct resonator-type cases. 
- Distributing the resonator frequencies over a higher frequency range within the bandwidth of interest is shown to give a relatively poorer performance.

- From a sequencing perspective it is found that having the low frequency resonator placed near the incident side gives the best performance.

- Engineering a high degree of impedance mismatch by sandwiching a unit with relatively 'soft' host material stiffness between two sections of units with relatively 'hard' host material stiffness enhances the overall attenuation performance provided by resonator action.

From a practical perspective, assonant mechanisms are found to play a significant role in determining the attenuation performance of an $\mathrm{AMB}$ when design constraints relevant to applications such as prescribed geometry, material, number of resonators, and number of sets of resonator frequencies are considered. In a similar vein, the term 'mechanical assonance' can be ascribed to the dynamic phenomenon realized by sequencing oscillators with tuned responses an energy harvesting application for mechanical assonant acoustic metamaterial was investigated. The derivation and code to calculate translational EMF and magnetic field was verified by comparing theoretical to experimental studies within a waveguide to engineer a prescribed wave transformation across it.

In the $4^{\text {th }}$ chapter, the investigation of linear electromechanical energy harvesting application for mechanically assonant acoustic metamaterial was demonstrated. The derivation and code to calculate translational EMF and magnetic field was verified by comparing theoretical to experimental studies. Some notable results that were found are:

- The coil configuration and position relative to the location of the magnet is seen to affect the frequency of the harvested voltage.

- For co-located and mirror coil configuration, the harvested voltage's frequency is seen to be doubles of the excitation frequency. This is expected as the magnetic 
field around the coils change twice in just half a cycle of the magnet's displacement.

- In the case of mirror coil configuration, the $52 \mathrm{~Hz}$ component harvested by the pair of coils positioned above and below the origin are 90 degree out of phase, thus canceling each other out. It can be possible to avoid this by either using phase adjustment or rectification.

- Overall, the semi-analytical model estimates the EMF with less percentage error than the proposed theoretical model. Thus, verifying the assumptions considered in section 4.4.1.1 and 4.4.1.2. The theoretical model assumes damping value, spring stiffness and mass which affects the accuracy of the displacement response of the magnet. Whereas for the case of semi-analytical the magnet's response is measured using a laser vibrometer during the experiment.

- It can be concluded that the best test case for EMF prediction (SIC-58) estimates the harvested signal within $2.4 \%$ error from the actual data. Moreover, the semianalytical interspersed configuration (SIC) predicts the generated voltage with $(<10 \%)$ error.

- Mirror and interspersed coil configuration studies, which use Equation (4.6) yield better prediction of EMF than the co-located assumption given in Equations (4.5). Equation (4.6) estimates the total generated voltage by summing the induced voltage for each coil, whereas Equation (4.5) assumes that all coils are co-located and can be multiplied by $\left(N_{\text {coils }}\right)$ to yield total EMF.

Energy harvesting for a one-dimensional AMB by assuming a single loop of wire positioned at the center of each unit was proposed and studied. It is found that:

- The harvested EMF for each unit cell is shown to decreases across the AMB, which is contributed by the wave attenuation from the preceding resonators. 
- The best performance for harvested voltage is yielded by the material variation case (SM6). It is seen that majority of the harvested voltage in the first 'hard' material region, then a significant decrease in voltage can be seen after the soft material which is mainly due to the high degree of impedance mismatch.

- From the perspective of sequencing and resonator variation (SQ6 and SR14) the harvested voltages results seem close to one another. Thus suggesting, sequencing doesn't have major influence in the performance of the harvester.

- The rectification of an AC voltage to DC was studied by comparing two ideal methods which were, adding all the AC voltages from each unit cell then rectifying (defined as sum-rectify), and rectifying each voltage harvested per unit cell then taking the summation to get the total DC (defined as rectify-sum). This study showed that rectifying prior to connecting each wire gives the higher output in DC voltage.

- For SR14 and SQ6 over 50\% decrease in DC voltage can be seen for the case of sum-rectify compared to rectify-sum technique. For SM6 the rectified signal for both defined techniques lie within less than $10 \%$ of each other as the phase difference between each resonator is seen to be very low.

\section{$\underline{5.2 \text { Future Recommendations }}$}

The history of humankind has always proven that there is a constant need for improved structural materials. In the pursuit of this drive there have been many major accomplishments when it comes to the study of materials, specifically acoustic metamaterials. Designs of artificial materials like AM has seen a great deal of interest in the aerospace and automotive industry. This thesis is aimed to explore a dual-functional mechanically assonant acoustic metamaterial in which different material variations were chosen to find an optimal choice using the idea of mechanical assonance. From this study some interesting patterns were observed for instance, octave spacing; 
this kind of patterns could be studied with the utilization of machine learning. From the perspective of optimization, genetic algorithms (GAs) can be used to find the best material and resonator combination to increase attenuation performance. To minimize the design space or number of factors that go in the GA, the defined two-parameter study can be used as mapping tool for the GA code. Further, correlation with impact-type experiments are recommended to refine the modeling of assonant mechanisms. It is expected that the results and observations from this study can provide preliminary guidelines leading towards optimal designs for resonant metamaterials as well as serve as a starting point for further, more formal optimization studies that yield quantitative statements regarding the assonant mechanisms involved. 


\section{REFERENCES}

1. Liu, Z., Zhang, X., Mao, Y., Zhu, Y. Y., Yang, Z., Chan, C. T.: Locally resonant sonic materials. Science, 289, 1734-1736, (2000).

2. Huang, G. L. and Sun, C. T.: Band gaps in a multiresonator acoustic metamaterial. Journal of Vibration and Acoustics, 132, (2010).

3. Zhu, R., Liu, X. N., Hu, G. K., Sun, C. T., and Huang, G. L.: A chiral elastic metamaterial beam for broadband vibration suppression. Journal of Sound and Vibration, 333, 2759-73 (2014).

4. Veselago, V. G.: Electrodynamics of media with simultaneously negative electric permittivity and magnetic permeability. Advances in Electromagnetics of Complex Media and Metamaterials, 90, 83-97 (2002).

5. Pendry, J. B., Holden, A. J., Stewart, W. J. and Youngs, I.: Extremely low frequency plasmons in metallic mesostructures. Physical Review Letters, 76, (1996).

6. Sui, Q. and Fang, F.: Experimental study of composite medium with simultaneously negative permeability and permittivity. Science in China Series G, 47, (2004).

7. Norris, A.: Generalized acoustic cloaking theory. The Journal of the Acoustical Society of America, 124, (2008).

8. Cummer, S. A. and Schurig, D.: One path to acoustic cloaking. New Journal of Physics, 9, (2007).

9. Li, J., and Chan, C. T.: Double-negative acoustic metamaterial. Physical Review E, 70, (2004). 
10. Huang, H. H., Sun, C. T., and Huang, G. L.: On the negative effective mass density in acoustic metamaterials. International Journal of Engineering Science, 47, 610-617, (2009).

11. Sheng, P., Mei, J., Liu, Z., and Wen, W.: Dynamic mass density and acoustic metamaterials. Physica B: Condensed Matter, 394, 256-61 (2007).

12. Fang, N., Xi, D., Xu, J., and Ambati, M.: Ultrasonic metamaterials with negative modulus. Nature Materials, 5, 452-456, (2006).

13. Mei, J., Liu Z., Wen, W., and Sheng, P.: Effective mass density of fluid-solid composites. Physical Review Letters, 96, (2006).

14. Kushwaha, M. S., Halevi, P., Dobrzynski, L., and Djafari-Rouhani, B.: Acoustic band structure of periodic elastic composites. Physical Review Letters, 71, (1993).

15. Martinsson, P. G., and Movchan, A. B.: Vibrations of lattice structures and phononic band gaps. The Quarterly Journal of Mechanics and Applied Mathematics, 56, 45-64 (2003).

16. Croënne, C., Lee, E. J. S., Hu, H., and Page J. H.: Band gaps in phononic crystals: generation mechanisms and interaction effects. AIP Advances, 1, (2011).

17. Yang, S., Page, J. H., Liu, Z., Cowan, M. L., Chan, C. T., and Sheng, P.: Focusing of sound in a 3D phononic Crystal. Physical Review Letters, 93, (2004).

18. Milton, G. W., and Willis, J. R.: On modifications of newton's second Law and linear continuum electrodynamics. Proceedings of the Royal Society A: Mathematical, Physical and Engineering Sciences, 463, 855-880 (2007). 
19. Vincent, J. H.: On the construction of a mechanical model to illustrate Helmholtz's theory of dispersion. The London, Edinburgh, and Dublin Philosophical Magazine and Journal of Science, 46, 557-536, (1898).

20. Yao, S., Zhou, X., and Hu, G.: Experimental study on negative mass effective mass in a 1D mass-spring system. New Journal of Physics, 10, (2008).

21. Huang, H. H, and Sun, C. T.: Wave attenuation mechanism in an acoustic metamaterial with negative effective mass density. New Journal of Physics, 11, (2009).

22. Caloz C.: Perspectives on EM metamaterials. Materials Today, 12, 12-20, (2009).

23. Ma, G., and Sheng, P.: Acoustic metamaterials: From local resonances to broad horizons. Science Advances, 2, (2016).

24. Sugino, C., Leadenham, S., Ruzzene, M., and Erturk, A.: On the mechanism of bandgap formation in locally resonant finite elastic metamaterials. Journal of Applied Physics, 120, (2016).

25. Kulkarni, P. P., and Manimala, J. M.: Longitudinal elastic wave propagation characteristics of inertant acoustic metamaterials." Journal of Applied Physics, 119, (2016).

26. Chen, Y. Y., Barnhart, M. V., Chen, J. K., Hu, G. K., Sun, C. T., and Huang G. L.: Dissipative elastic metamaterials for broadband wave mitigation at subwavelength scale. Composite Structures, 136, (2016).

27. Fang, X., Wen, J., Bonello, B., Yin, J., and Yu, D.: Ultra-low and ultra-broad-band nonlinear acoustic metamaterials. Nature Communications, $\mathbf{8 , ~ ( 2 0 1 7 ) . ~}$

28. Kinney, F. G, and Graham, J. K.: Explosive shocks in air ( $2^{\text {nd }}$ ed.)., Springer, (1985).

29. Christou, G. A., Young, L. R., Goel, R., Vechart, A. P., and Jerusalem, A.: Shock attenuation of PMMA sandwich panels filled with soda-lime glass beads: A fluid-structure interaction continuum model simulation. International Journal of Impact Engineering, 47, 48-59 (2012). 
30. Su, Z., Peng, W., Zhang, Z., Gogos, G., Skaggs, R., and Cheeseman, B.: Numerical simulation of a novel blast wave mitigation device. International Journal of Impact Engineering, 35, 336-46 (2008).

31. Grujicic, M., Bell, W. C., Pandurangan, B., and He, T.: Blast-wave impact-mitigation capability of polyurea when used as helmet suspension-pad material. Material \& Design, 31, 4050-4065, (2010).

32. Tan, K. T., Huang, H. H., and Sun, C. T.: Blast-wave impact mitigation using negative effective mass density concept of elastic metamaterials. International of Journal Impact Engineering, 64, 20-29, (2014).

33. Ma, G., Yang, M., Xiao, S., Yang, Z., and Shen, P.: Acoustic metasurface with hybrid resonances. Nature Materials, 13, 873-78 (2014).

34. Gonella, S., To, A. C., and Liu, W. K.: Interplay between phononic bandgaps and piezoelectric microstructures for energy harvesting. Journal of the Mechanics and Physics of Solids, 57, 621-633 (2009).

35. Beeby, S. P., Tudor, M. J., and White, N. M.: Energy harvesting vibration sources for microsystems application. Measurements Science and Technology, 17, 172-195 (2006).

36. Mikoshiba, K., Manimala, J. M., and Sun, C. T., "Energy harvesting using an array of multifunctional resonators." Journal of Intelligent Material Systems and Structures, 24, 168-179, (2012).

37. Carrara, M., Cacan, M. R., Toussaint, J., Leamy, M. J., Ruzzene, M., and Erturk, A.: Metamaterial-inspired structures and concepts for elastoacoustic wave energy harvesting. Smart Materials and Structures, 22, (2013).

38. Nguyen, H., Zhu, R., Chen, J. K., Tracy, S. L., and Huang, G. L.: Analytical coupled modeling of a magneto-based acoustic metamaterial harvester. Smart Materials and Structures, 27, (2018). 
39. Erturk, A., and Inman, D. J.: Piezoelectric Energy Harvesting. Wiley, (2011).

40. Ramadan, K. S, Sameoto, D., and Evoy, S.: A review of piezoelectric polymers as functional materials for electromechanical transducers. Smart Materials and Structures, 23, (2014).

41. Kim, H. S., Kim, J. H., and Kim, J.: A review of piezoelectric energy harvesting based on vibration. International Journal of Precision Engineering, 12, 1129-1141 (2011).

42. Smith, D. R., Padilla, W. J., Vier, D. C., Nemat-Nasser, S. C., and Schultz, S.: Composite medium with simultaneously negative permeability and permittivity. Physical Review Letters, 84, 4184-4187 (2000).

43. Sugino, C., Xia Y., Leadenham, S., Ruzzene, M., and Erturk, A.: A general theory for bandgap estimation in locally resonant metastructures. Journal of Sound and Vibration, 406, (2017).

44. Tan, K. T., Huang, H. H., and Sun, C. T.: Optimizing the band gap of effective mass negativity in acoustic metamaterial. Journal of Applied Physics, 101, (2012).

45. Johnson, W. R., and Ruzzene, M.: Challenges and constraints in the application of resonance-based metamaterials for vibration isolation. Health Monitoring of Structural and Biological Systems XII, (2018).

46. Caciagli, A., Baars, R. J., Philipse, A. P, and Kuipers, B. W. M.: Exact xpression for the magnetic field of a finite cylinder with arbitrary niform magnetization. Journal of Magnetism and Magnetic Materials, 456, (2018).

47. Derby, N., and Olbert, S.: Cylindrical magnets and ideal solenoids. American Journal of Phsyics, 78, 229-235, (2010).

48. Tan, T., Yan, Z., Zou, H., Ma, K., Liu, F., Zhao, L., Peng, Z., and Zhang, W.: Renewable energy harvesting and absorbing via multi-scale etamaterial systems for internet of things. Applied Energy, 254, (2019). 
49. Yasuda, H., Miyazawa, Y., Charalampidis, E. G., Chong, C., Kevrekidis, P. G., and Yang, J.: Origami-based impact mitigation via rarefaction solitary wave creation. Science Advances, 5, (2019).

50. Shan, S., Kang, S. H., Raney, J. R., Wang, P., Fang, L., Candido, F., Lewis, J. A., and Bertoldi, K.: Multistable architected materials for trapping elastic train energy. Advanced Materials, 27, 4296-4301 (2015).

51. Hiraiwa, M., Abi Ghanem, M., Wallen, S. P., Khanolkar, A., Maznev, A. A., and Boechler, N.: Complex contact-based dynamics of microsphere monolayers revealed by resonant ttenuation of surface acoustic waves. Physics Review Letter, 116, (2016).

52. Raney, J. R., Nadkarni, N., Daraio, C., Kochmann, D. M., Lewis, J. A., and Bertoldi K.: Stable propagation of mechanical signals in soft media using stored elastic energy. Proccedings of the National Academy of Science, 113, 9722-9727 (2016).

53. Starosvetsky, Y., Jayaprakash, K. R., Hasan, M. A., and Vakakis, A. F.: Dynamics and acoustics of ordered granular media. World Scientific, (2017).

54. Silverberg, J. L., Evans, A. A., McLeod, L., Hayward, R. C., Hull, T., Santangelo, C.D., and Cohen, I.: Using origami design principles to fold reprogrammable mechanical metamaterials. Science, 345, 647-650 (2014).

55. Lv, C., Krishnaraju, D., Konjevod, G., Yu, H., Jiang, H.: Origami based mechanical metamaterials. Scientific Reports, 4, (2014).

56. Waitukaitis, S., Menaut, R., Chen, B. G., and Van Hecke M.: Origami multistability: From single vertices to metasheets. Physics Review Letter, 114, (2015).

57. Overvelde, J. T. B., de Jong, T. A., Shevchenko, Y., Becerra, S. A., Whitesides G. M., Weaver J. C., Hoberman C., Bertoldi K.: A three-dimensional actuated origami-inspired transformable metamaterial with multiple degrees of freedom. Nature Communication, $\mathbf{7}$, (2016). 
58. Liu, K., Paulino, G. H.: Nonlinear mechanics of non-rigid origami: An efficient computational approach. Proceedings of the Royal Society A: Mathematical, Physical and Engineering Sciences, 473, (2017).

59. Filipov, E. T., Tachi, T., Paulino, G. H.: Origami tubes assembled into stiff, yet reconfigurable structures and metamaterials. Proceedings of the National Academy of Sciences, 112, 12321-12326 (2015).

60. Yasuda, H., Yang, J.: Reentrant origami-based metamaterials with negative Poisson's ratio and bistability. Physics Review Letter, 114, (2015).

61. Hanna, B. H., Lund, J. M., Lang, R. J., Magleby, S. P., Howell, L. L.: Waterbomb base: A symmetric single-vertex bistable origami mechanism. Smart Material and Strucures, 23, (2014).

62. Jianguo, C., Xiaowei, D., Ya, Z., Jian, F., Yongming, T.: Bistable behavior of the cylindrical origami structure with Kresling pattern. Journal of Mechanical Design, 137, (2015).

63. Yasuda, H., Tachi, T., Lee, M., Yang, J.: Origami-based tunable truss structures fornonvolatile mechanical memory operation. Nature Communications, 8 , (2017).

64. Lakes, R. S., Lee, T., Bersie, A., and Wang, Y. C.: Extreme damping in composite materials with negative-stiffness inclusions. Nature, 410, (2001).

65. Manimala, J. M., Huang H. H., Sun C. T., Snyder R., and Bland S.: Dynamic load mitigation using negative effective mass structures. Engineering Structures, 80, (2014).

66. https://www.atlasobscura.com/places/tuned-mass-damper-of-taipei-101

67. https://wordsworth.org.uk/wordsworth/daffodils-and-other-poems/wordsworths-daffodils/

68. https://www.wsdot.wa.gov/TNBhistory/resources.htm

69. Lanz, H.: The physical basis of rime: An essay on the aesthetics of sound. The Philosophical Review, 42, 636-638 (1933). 
70. http://www.oxforddictionaries.com/us/definition/american english/assonance 


\section{APPENDICES}

\section{APPENDIX A: Python and MATLAB codes}

\section{A.1 Python - Parametric study}

from abaqus import $*$

from abaqusConstants import *

from caeModules import *

from odbAccess import *

import sys

from disp_reader import disp_reader

import numpy as np

openMdb('20_Unit_20x500Hz_SK1.cae') \# calling the abaqus model to open

keven $=[\#$ Insert Keven stiffness here separated by comma $]$ \# Stiffness in N/m kodd=[\#Insert Kodd stifness here separated by comma]

omega $=[\#$ Insert omega separated by a comma];

no_of_jobs = \# Enter number of simulation to be carried out here

name_jobs $=[0] *$ no_of_jobs

for io in range(no_of_jobs):

name_jobs[io]='SK' + str(io+1)

$\mathrm{n}=$ len(keven)

$\mathrm{m}=$ len(omega)

for $i$ in range $(m)$ :

for $\mathrm{j}$ in range( $\mathrm{n})$ :

mdb.models['Model-

1'].rootAssembly.engineeringFeatures.springDashpots['K_Hard'].setValues( springStiffness $=$ keven[j]) 


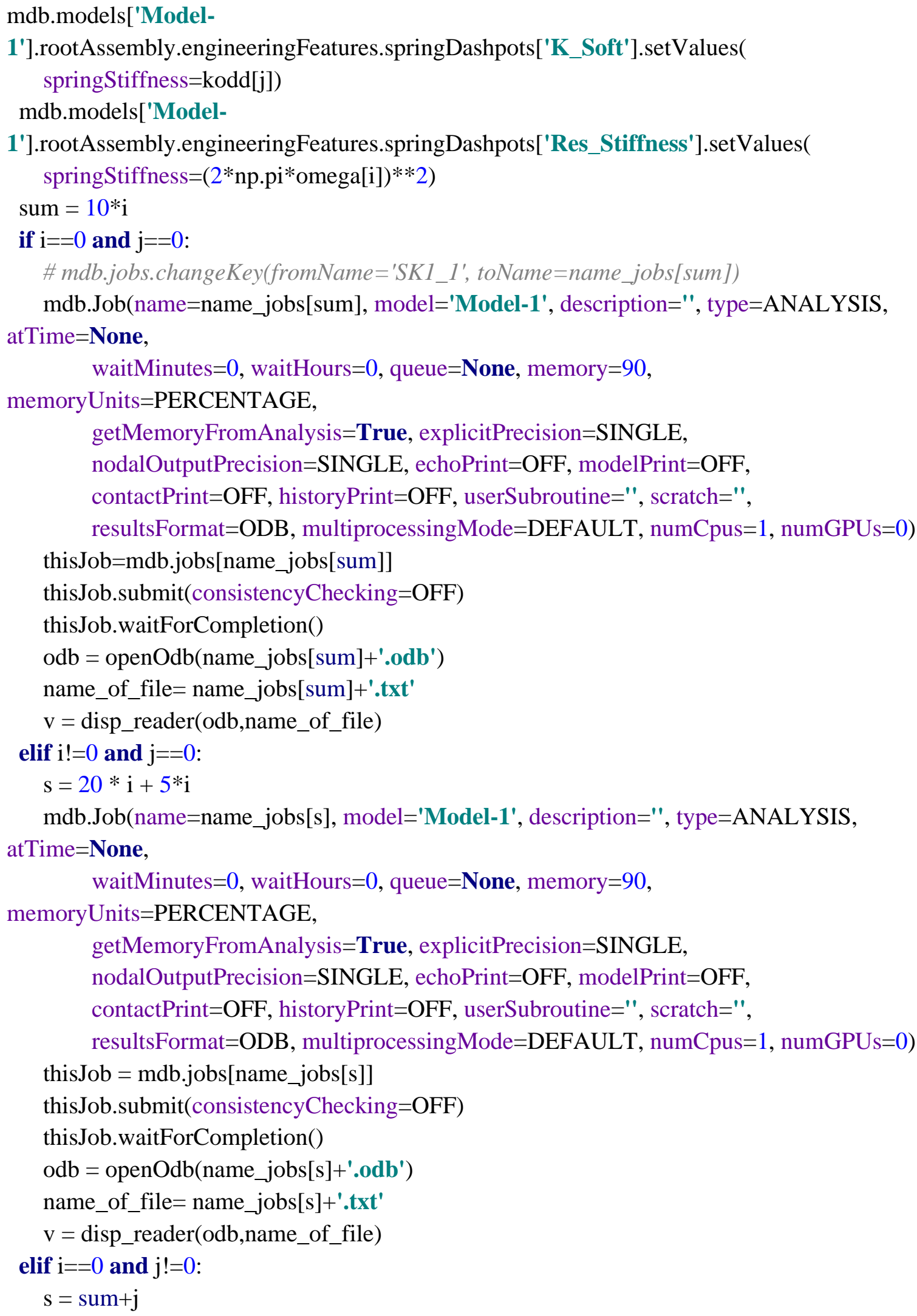


mdb.Job(name=name_jobs[s], model='Model-1', description="', type=ANALYSIS, at Time $=$ None,

waitMinutes $=0$, waitHours $=0$, queue $=$ None, memory $=90$,

memoryUnits=PERCENTAGE,

getMemoryFromAnalysis=True, explicitPrecision=SINGLE,

nodalOutputPrecision $=$ SINGLE, echoPrint $=\mathrm{OFF}$, modelPrint $=\mathrm{OFF}$,

contactPrint $=\mathrm{OFF}$, historyPrint $=\mathrm{OFF}$, userSubroutine $="$ ", scratch $="$,

resultsFormat $=\mathrm{ODB}$, multiprocessingMode $=$ DEFAULT, numCpus $=1$, numGPUs $=0$ )

thisJob $=$ mdb.jobs[name_jobs[s]]

thisJob.submit(consistencyChecking=OFF)

thisJob.waitForCompletion()

odb $=$ openOdb $\left(\right.$ name_jobs $[\mathrm{s}]+$ '.odlb' $\left.^{\prime}\right)$

name_of_file $=$ name_jobs $[\mathrm{s}]+$ '.txt' $^{\prime}$

$\mathrm{v}=$ disp_reader(odb, name_of_file)

else:

mdb.Job(name=name_jobs[s+j], model='Model-1', description=" , type=ANALYSIS, at Time $=$ None,

waitMinutes $=0$, waitHours $=0$, queue $=$ None, memory $=90$, memoryUnits=PERCENTAGE,

getMemoryFromAnalysis=True, explicitPrecision=SINGLE, nodalOutputPrecision $=$ SINGLE, echoPrint $=\mathrm{OFF}$, modelPrint $=\mathrm{OFF}$, contactPrint $=\mathrm{OFF}$, historyPrint $=\mathrm{OFF}$, userSubroutine $="$ ", scratch $="$,

resultsFormat $=\mathrm{ODB}$, multiprocessingMode $=$ DEFAULT, numCpus $=1$, numGPUs $=0$ )

thisJob $=\operatorname{mdb} . j o b s[$ name_jobs $[\mathrm{s}+\mathrm{j}]]$

thisJob.submit( consistencyChecking=OFF)

thisJob.waitForCompletion()

odb $=$ openOdb $($ name_jobs $[s+j]+$ '.odlb')

name_of_file $=$ name_jobs $[\mathrm{s}+\mathrm{j}]+{ }^{\prime} . \mathrm{txt}^{\prime}$

$\mathrm{v}=$ disp_reader(odb, name_of_file) 


\section{A.2 MATLAB - Parametric study post processor}

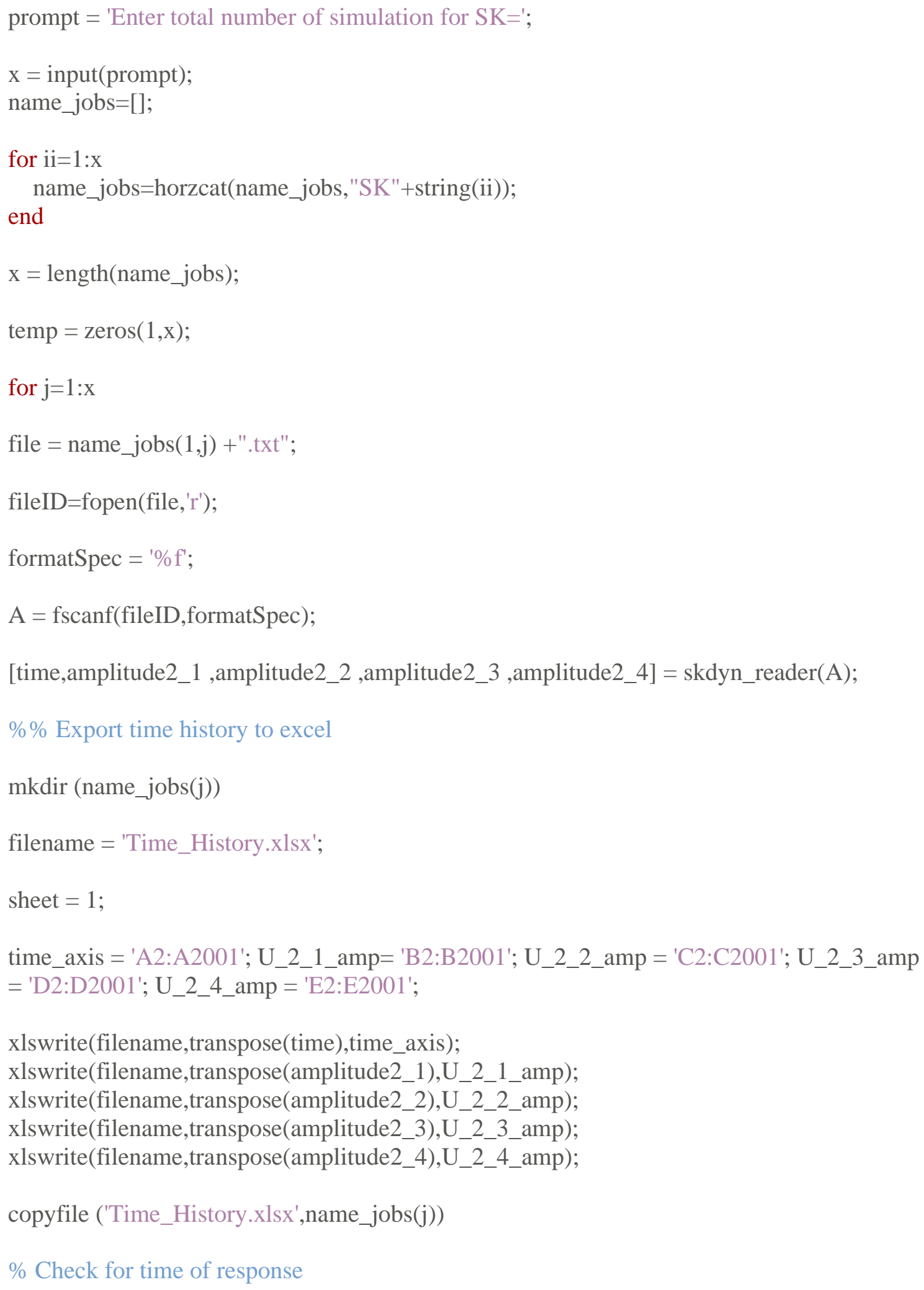




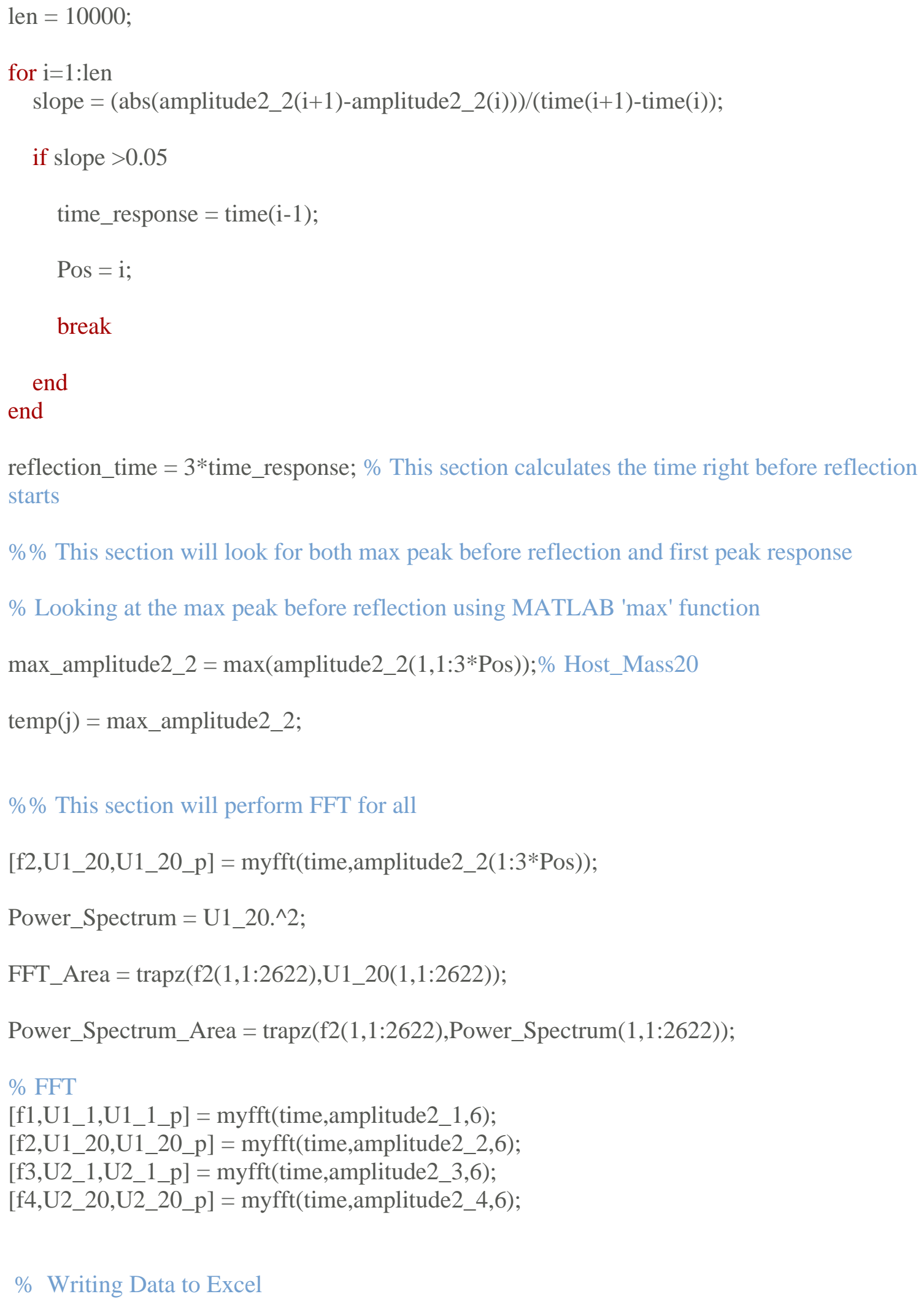


filename $=$ 'FFT.xlsx';

sheet $=1$;

time_axis = 'A2:A2001'; U_2_1_amp= 'B2:B2001'; U_2_2_amp = 'C2:C2001'; U_2_3_amp

= 'D2:D2001'; U_2_4_amp = 'E2:E2001';

max_res = 'M3'; nmax_res = 'L3';

xlswrite(filename,transpose(f2),time_axis);

xlswrite(filename, $\left.\operatorname{transpose}\left(\mathrm{U} 1 \_20\right), \mathrm{U} \_2 \_2 \_a m p\right)$;

xlswrite(filename, transpose(U2_1),U_2_3_amp);

xlswrite(filename, transpose(U2_20),U_2_4_amp);

xlswrite(filename,('M'),sheet,nmax_res);

xlswrite(filename,transpose(max_amplitude2_2),sheet,max_res);

copyfile ('FFT.xlsx',name_jobs(j))

end

$\% \%$ This section will create the surface plot after all the data parsing is complete

basevalue_displacment $=0.0202$;

d_star = temp/basevalue_displacment;

omega $=[]$

stiffness_ratio $=[]$;

$\mathrm{w}=2500$

normalized_omega $=$ omega/w;

$[\mathrm{X}, \mathrm{Y}]=$ meshgrid$($ stiffness_ratio,normalized_omega);

figure

$\operatorname{surf}(\mathrm{X}, \mathrm{Y}, \mathrm{d}$ _star$)$

colormap jet

colorbar

xlabel('Stiffness Ratio, kodd/keven')

ylabel('Normalized frequency')

zlabel('Normalized max peak before ref (D*)')

title('Two-parameter Surface Plot') 


\section{A.3 MATLAB - Import data from text file}

$\%$ txt file reader

function [time,U1_1,U1_20,Res_Mass_1,Res_Mass_20] = dyn_reader(A)

$\mathrm{A}=\operatorname{transpose}(\mathrm{A})$;

time $=0: 4 \mathrm{e}-4: 5$

$\mathrm{U} 1 \_1=\mathrm{A}(1: 2001)$

U1_20= A(2002:4002);

Res_Mass_1= A(4003:6003);

Res_Mass_20= A(6004:8004);

end 


\section{A.4 MATLAB - Experiment data post processor}

filename = 'Insert a $\cdot$ xlsx file here';

sheet $=1$;

time_axis = 'A2:A10001'; U_2_1_amp= 'C2:C10001'; vol= 'D2:D10001'; vol_R1= 'E2:E10001';vol_R2= 'F2:F10001';

$\%$ setting values to read from excel

time $=$ transpose $(x l s r e a d(f i l e n a m e$, sheet,time_axis $)) ; \%$ transposing to a column matrix acceleration $=1000 *(\operatorname{transpose}(x l s r e a d(f i l e n a m e$, sheet,U_2_1_amp $))) ; \%$ multiplying by a thousand to convert $\mathrm{m}$ to $\mathrm{mm}$

voltage_read $=(\operatorname{transpose}(\mathrm{xlsread}($ filename, sheet, $\mathrm{vol})))$;

$[\mathrm{f} 1$,accleration_fft $]=\operatorname{myfft}($ time, acceleration, 2$) ; \%$ taking the FFT of the acceleration data

Fs $=1 /($ time(2)-time(1)); \% sampling freq for acc

$\% \%$ Perform conversion of acceleration data to velocity.

$\mathrm{dt}=\operatorname{time}(2)-\operatorname{time}(1)$;

velocity_accelerometer $=\operatorname{trapz}($ acceleration $)$;

subplot $(2,1,1) \quad \%$ time history plots of velocity data

plot(time,velocity_accelerometer)

title('Converted Velocity Time History')

xlabel('Time, s')

ylabel('Velocity, mm/s')

[f_vel_acc,vel_acc] = myfft(time,velocity_accelerometer,2); \% taking the FFT of the acceleration data

$\% \%$ Read Laser Vibrometer data from excel file

vibrometer= 'B2:B10001';

lvelo $=$ transpose $(x l s r e a d($ filename, sheet, vibrometer $)) ; \%$ transposing to a column matrix

[f_vel_laser,vel_laser] = myfft(time,laser_velo,2); \% taking the FFT of the acceleration data

$\% \%$ The following section will perform the conversion of velocity to displacement using MATLAB inbuilt function 'cumtrapz' 


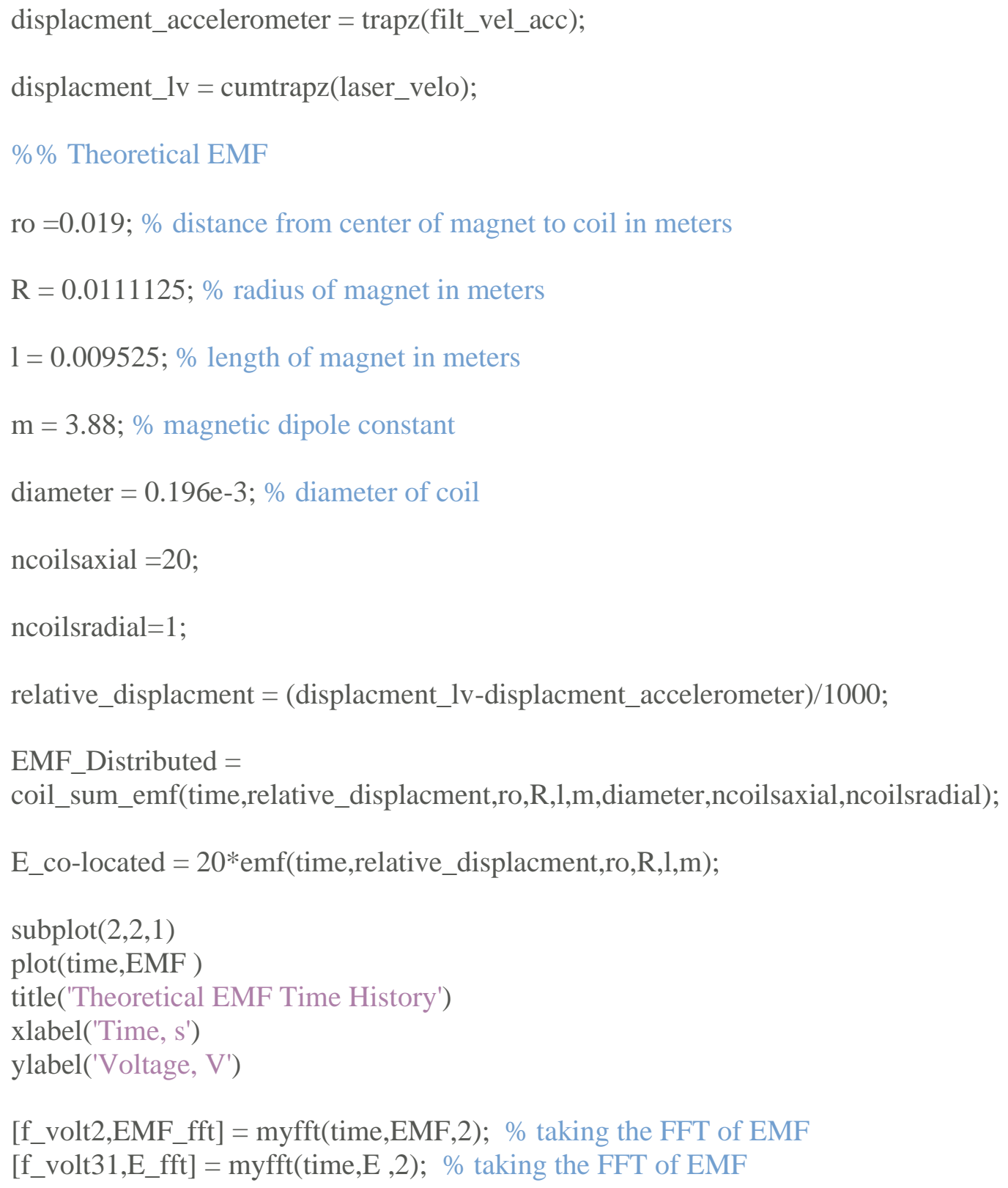




\section{A.5 MATLAB - Magnetic Field Plotter}

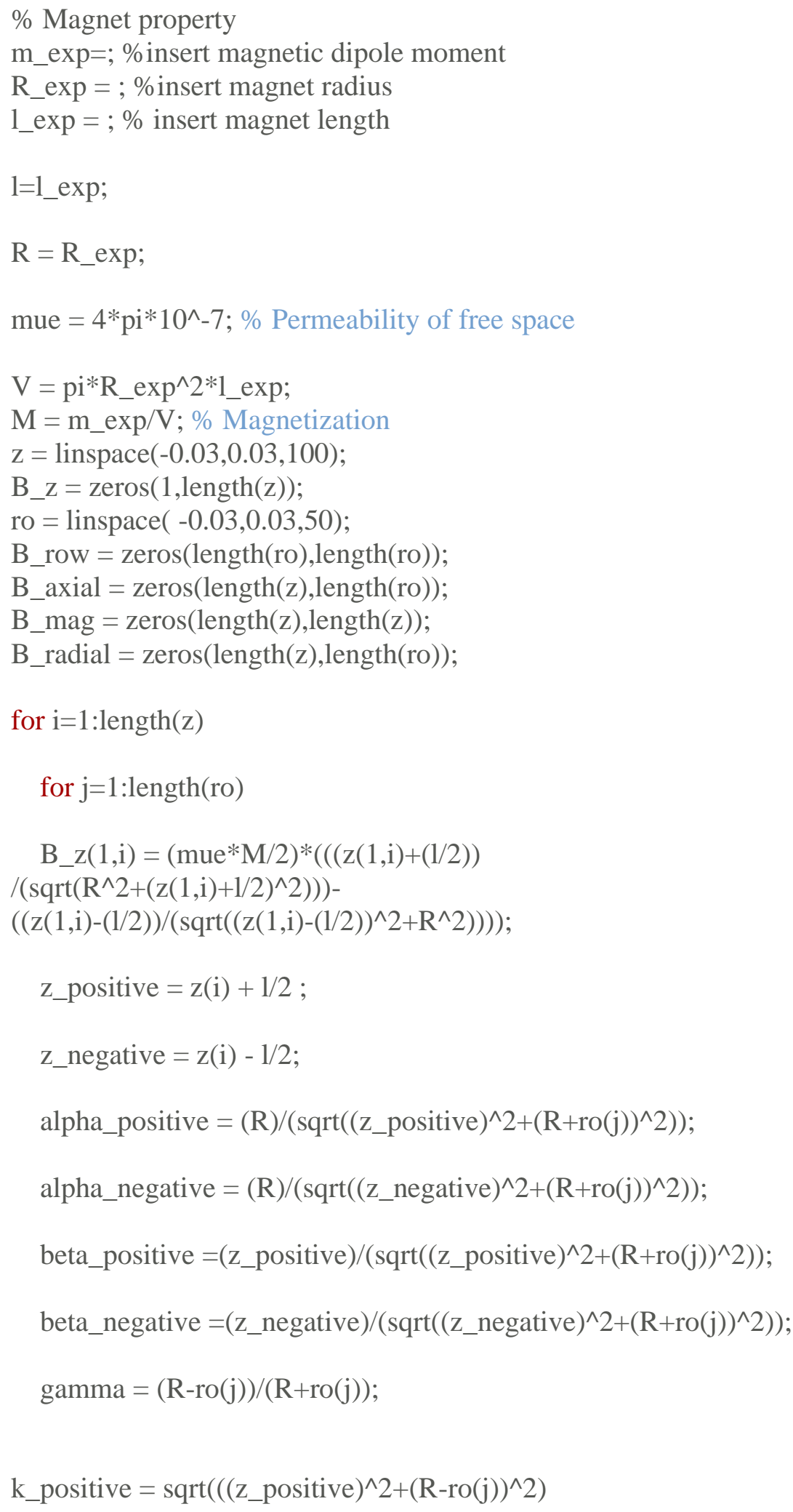




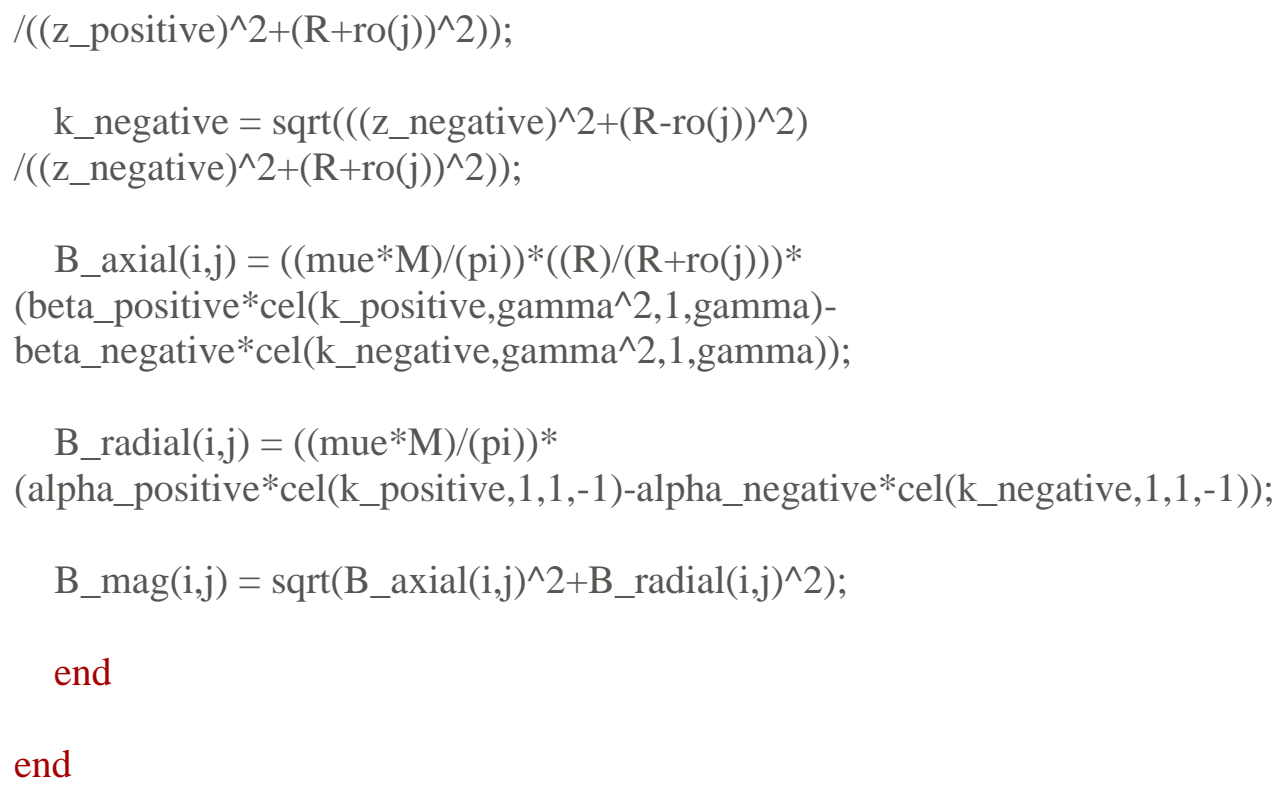




\section{A.6 MATLAB - Complete elliptic integral calculator}

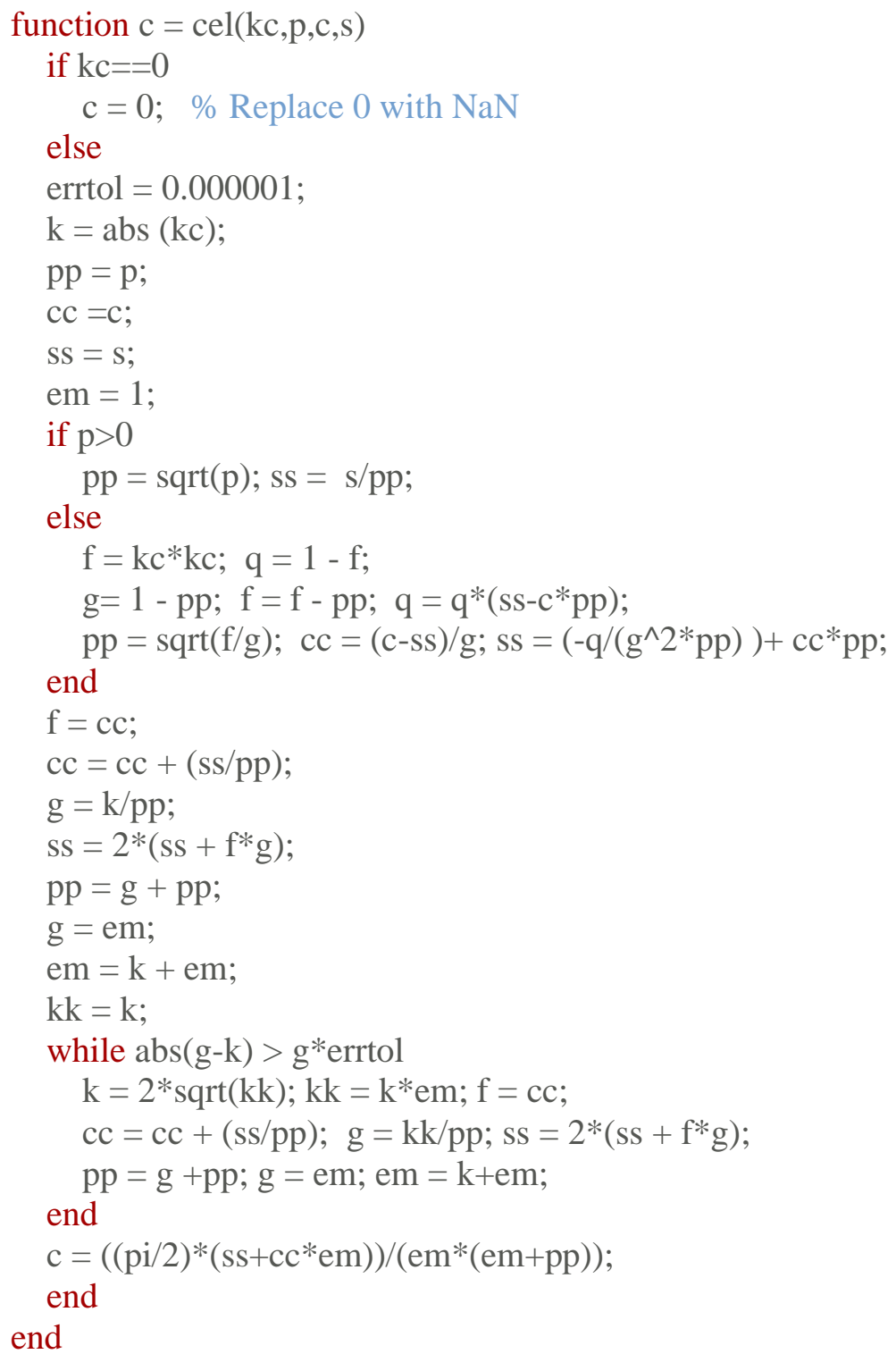




\section{A.7 MATLAB - EMF distributed coil}

function volt $=$ coil_sum_emf $($ time,disp,ro, R, $1, \mathrm{~m}$, diameter,ncoilsaxial,ncoilsradial $)$

$\%$ diameter of coil, ncoils, number of coils, ro - radial distance from magnet, $\mathrm{R}$ - radius of magnet, 1-length of magnet, $\mathrm{m}$ - magnet dipole moment

$\mathrm{n}=$ length(time);

volt $=$ zeros $(1, \mathrm{n})$;

velocity $=$ myvelo(time, disp $)$;

[axialcoils,radialcoils] = coil_locator(ncoilsaxial,ncoilsradial,diameter);

for $\mathrm{i}=1: \mathrm{n}$

sumaxial $=0$;

sumradial $=0$;

if $i==1$

$\operatorname{volt}(1, \mathrm{i})=0$;

else

for $\mathrm{j}=1$ :ncoilsaxial

for $\mathrm{q}=1$ :ncoilsradial

temp $=(($ magnetic_flux_sum $(\operatorname{disp}(1, \mathrm{i}), \mathrm{ro}, \mathrm{R}, \mathrm{l}, \mathrm{m}, \operatorname{axialcoils}(\mathrm{j}), \operatorname{radialcoils}(\mathrm{q}))-$ magnetic_flux_sum(disp(1,i-1),ro,R,1,m,axialcoils(j),radialcoils(q)))/(disp(1,i) - disp(1,i$1)))^{*} \operatorname{velocity}(1, \mathrm{i}) * \mathrm{pi}^{*}(\operatorname{ro}+\operatorname{radialcoils}(\mathrm{q}))^{\wedge} 2$;

sumradial = sumradial+temp;

end

sumaxial=sumaxial+sumradial;

end

$\operatorname{volt}(1, \mathrm{i})=$ sumaxial;

end

end

end 


\section{A.8 MATLAB - Magnetic field to calculate EMF for distributed coils}

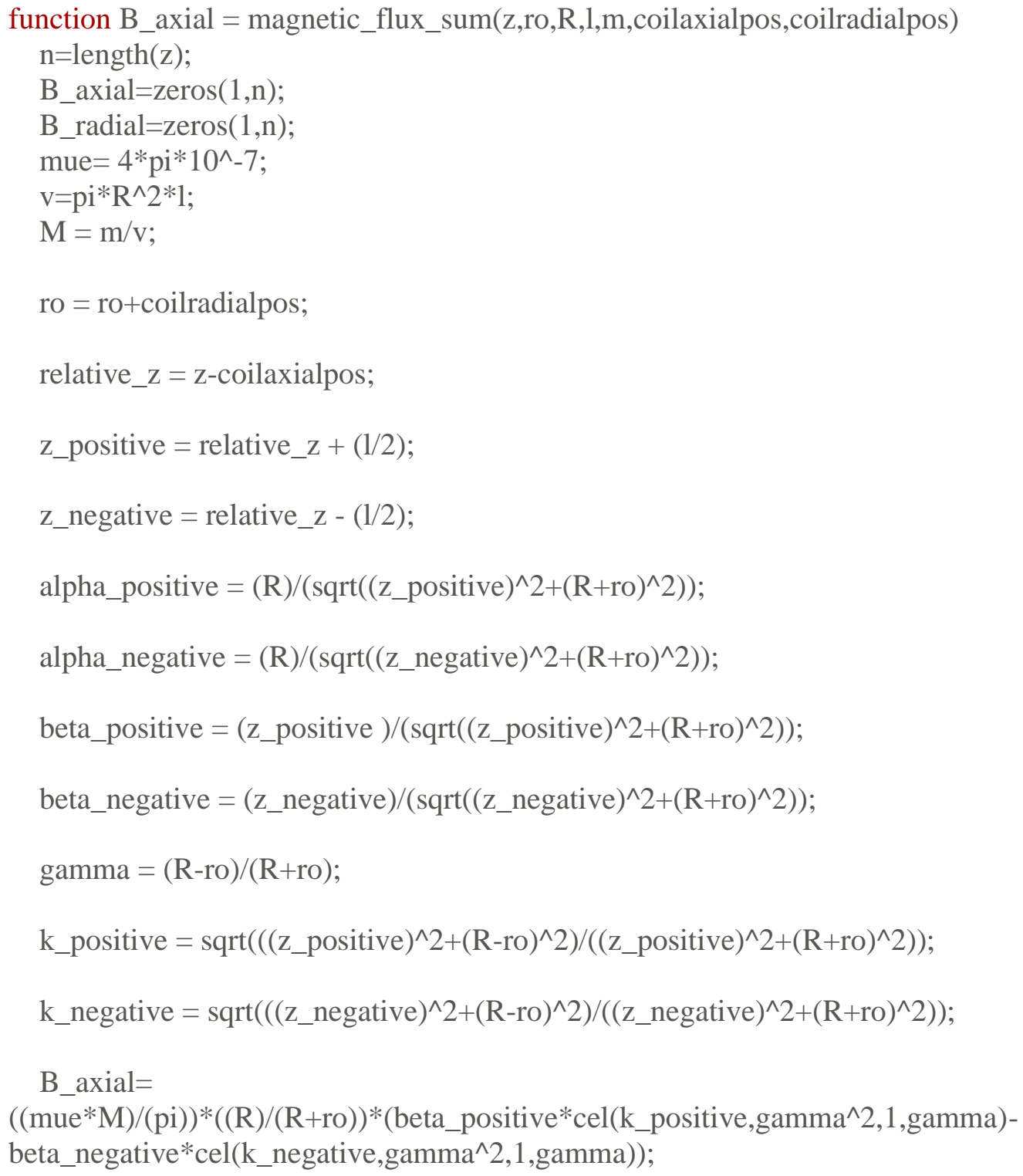

end 


\section{A.9 MATLAB - Coil position locator}

function [axial_coil_location, radial_coil_location] = coil_locator(naxialcoils,nradialcoils,diameter)

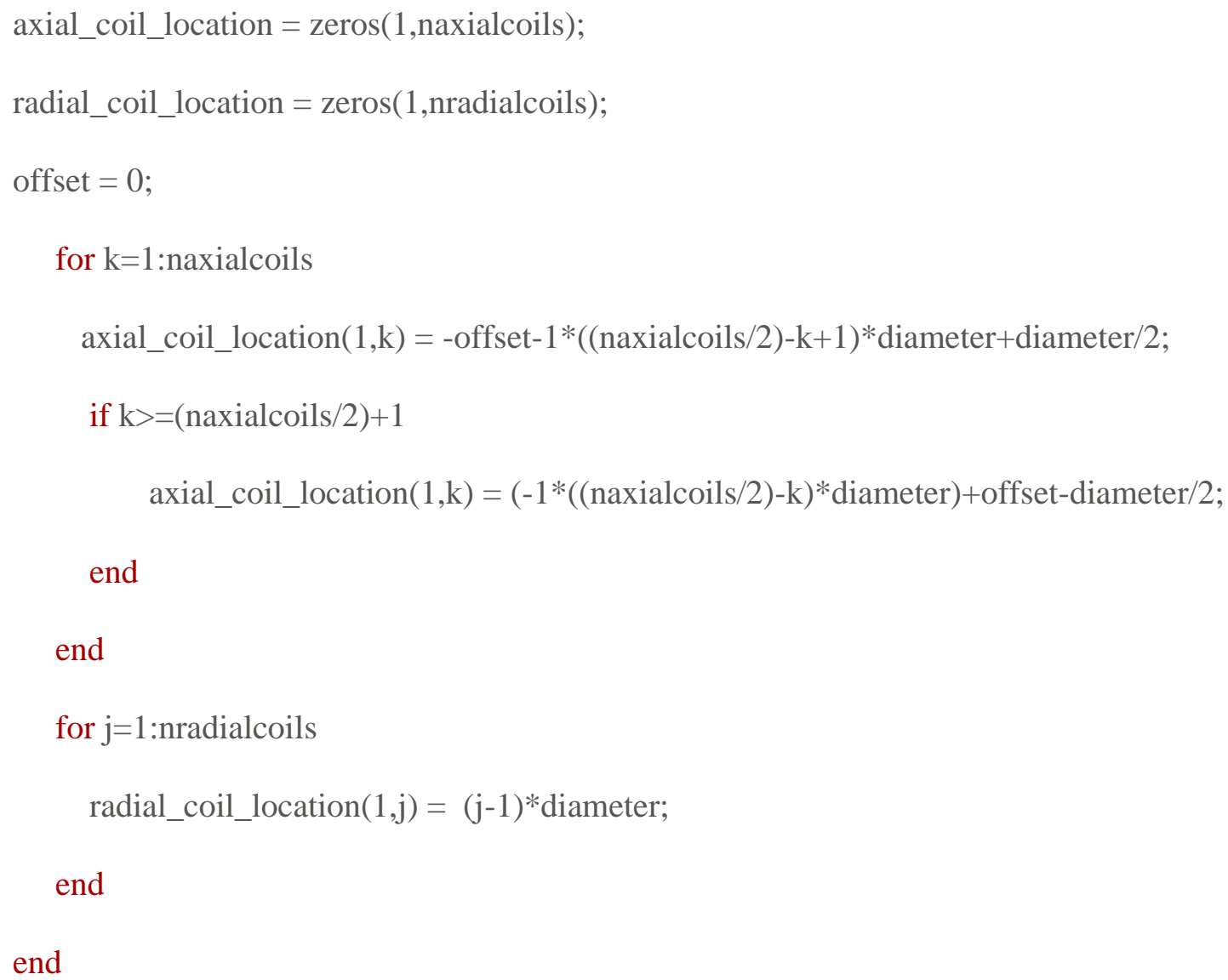


APPENDIX B: Additional Figures and Plots

\section{B.1 Charts for Co-Located Configuration}

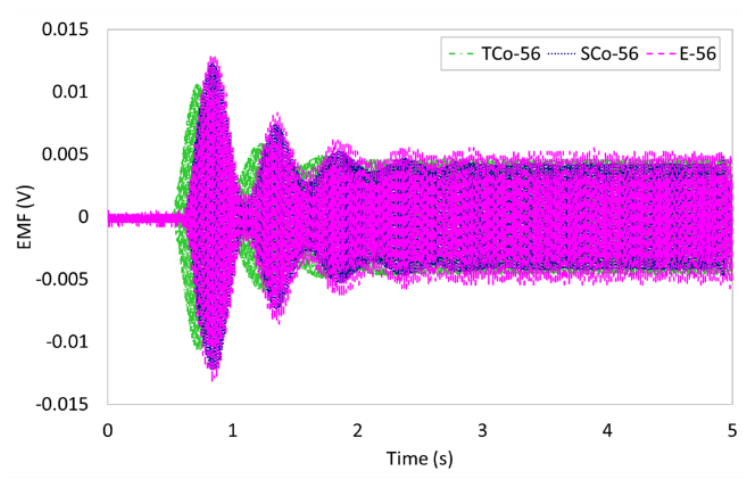

(a)

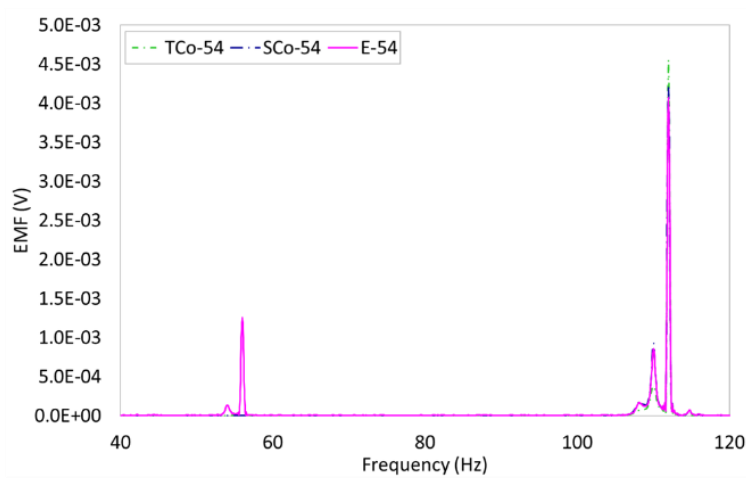

(b)

Figure B.1.1: EMF data, (a) time history and (b) frequency spectra plots for $56 \mathrm{~Hz}$ excitation input with co-located coil configuration.

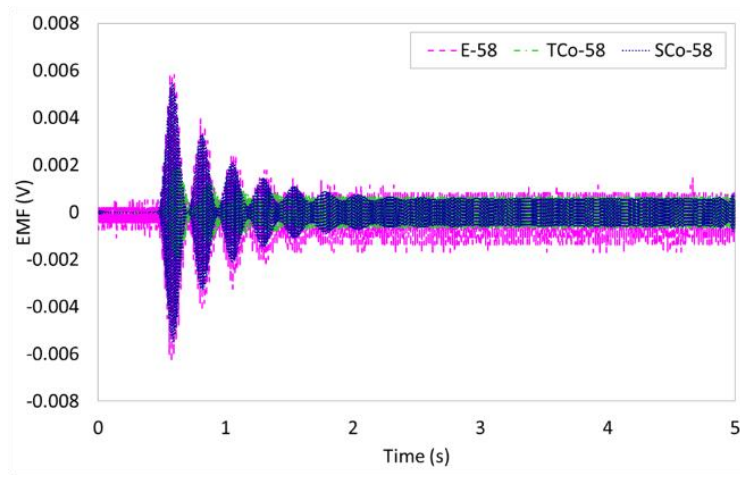

(a)

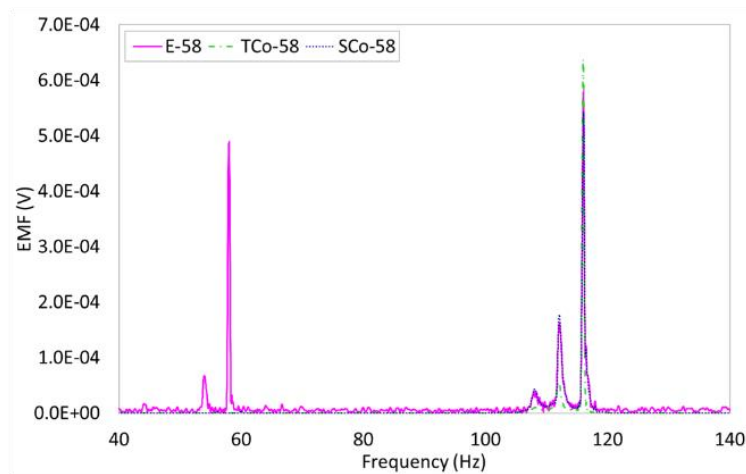

(b)

Figure B.1.2: EMF data, (a) time history and (b) frequency spectra plots for $58 \mathrm{~Hz}$ excitation input with co-located coil configuration. 


\section{B.2 Charts for Mirror Configuration}

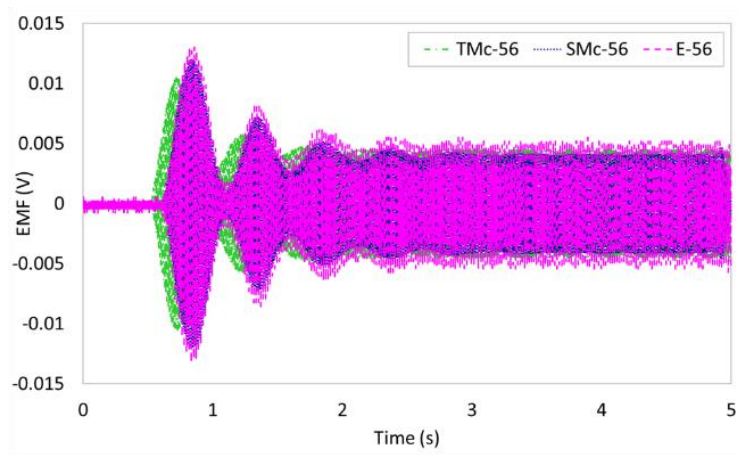

(a)

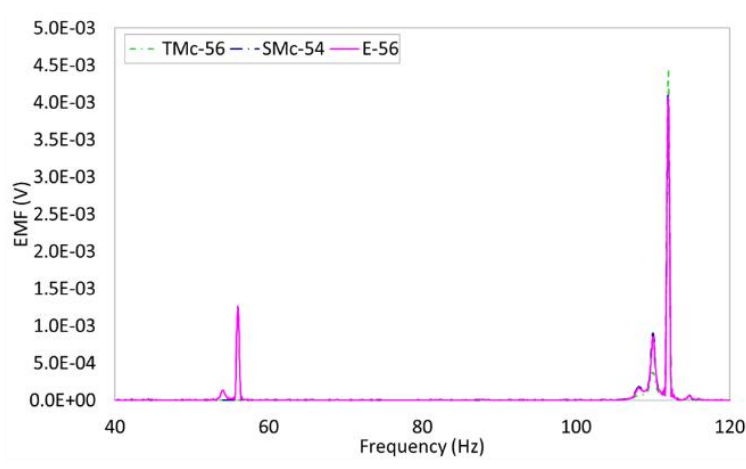

(b)

Figure B.2.1: EMF data, (a) time history and (b) frequency spectra plots for $56 \mathrm{~Hz}$ excitation input with mirror coil configuration.

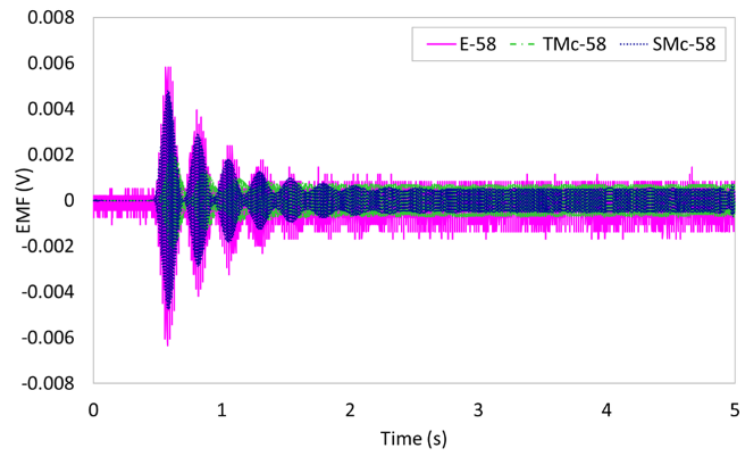

(a)

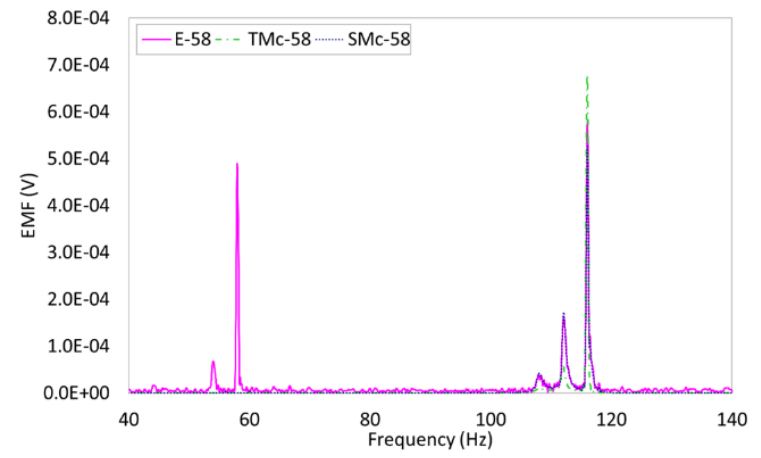

(b)

Figure B.2.2: EMF data, (a) time history and (b) frequency spectra plots for $58 \mathrm{~Hz}$ excitation input with mirror coil configuration. 


\section{B.3 Charts for interspersed Configuration}

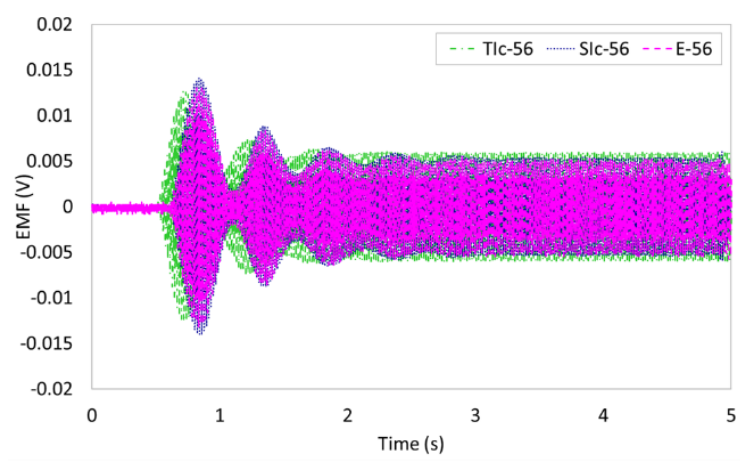

(a)

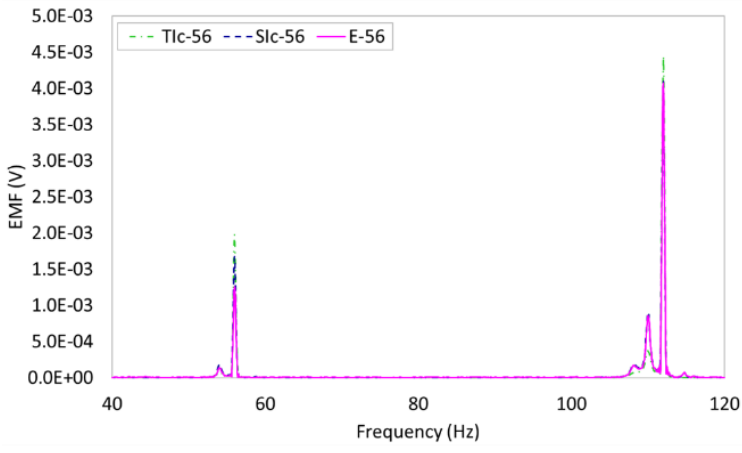

(b)

Figure B.3.1: EMF data, (a) time history and (b) frequency spectra plots for $56 \mathrm{~Hz}$ excitation input with interspersed coil configuration.

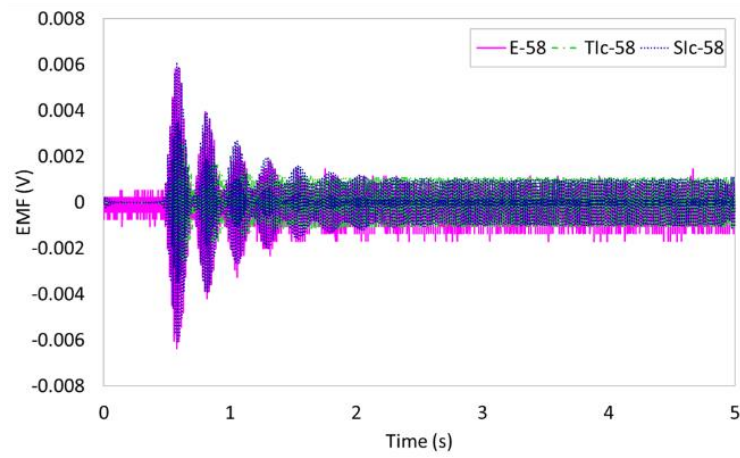

(a)

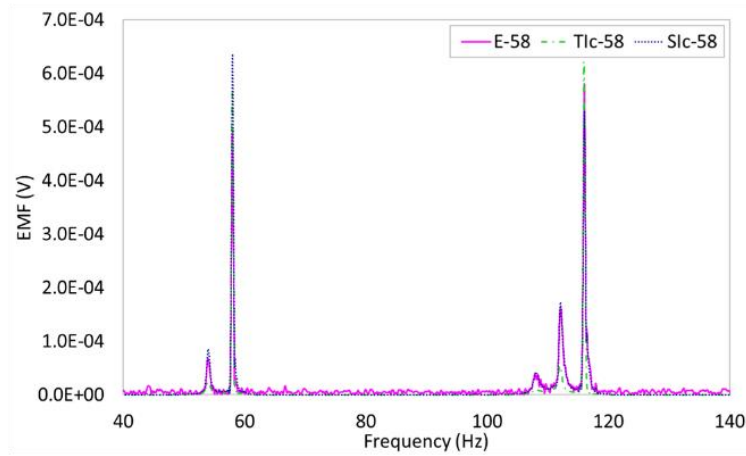

(b)

Figure B.3.2: EMF data, (a) time history and (b) frequency spectra plots for $58 \mathrm{~Hz}$ excitation input with interspersed coil configuration. 


\section{B.4 Displacement input and response for $52 \mathrm{~Hz}$ excitation}

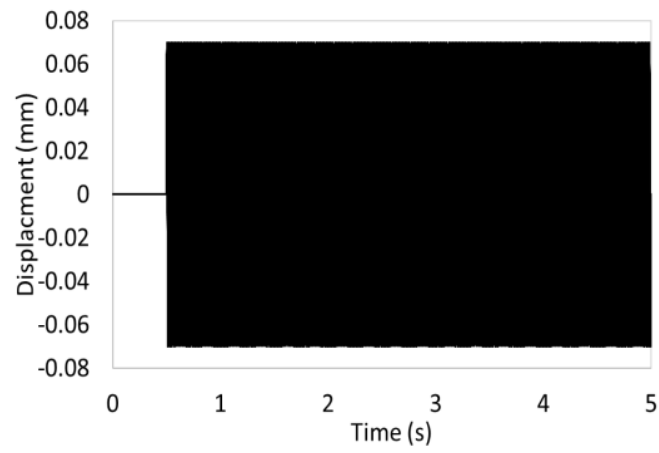

(a)

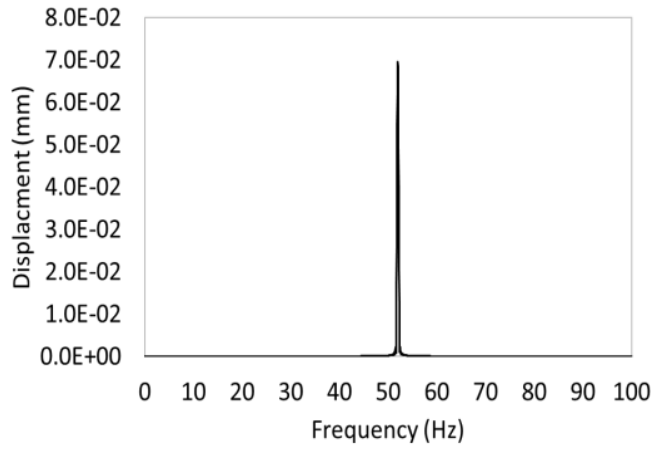

(b)

Figure B.4.1: Theoretical input displacement (a) time history and (b) frequency spectra for $52 \mathrm{~Hz}$ excitation.

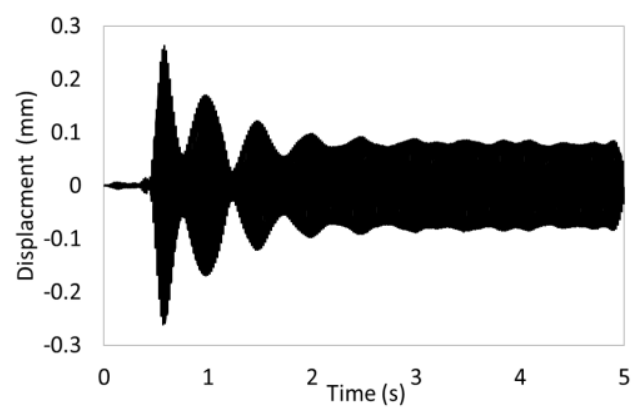

(a)

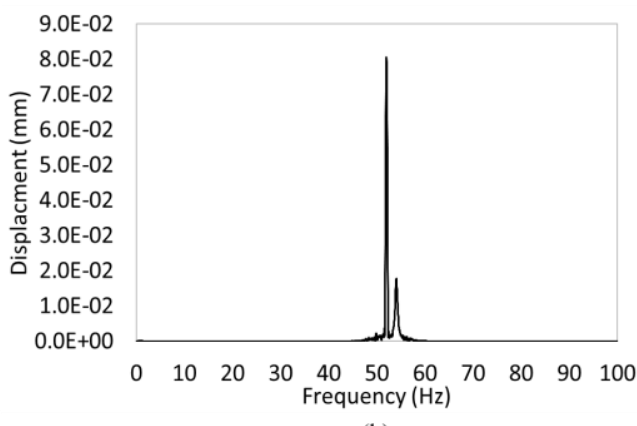

(b)

Figure B.4.2: Experimental input displacement (a) time history and (b) frequency spectra for 52

Hz excitation. 


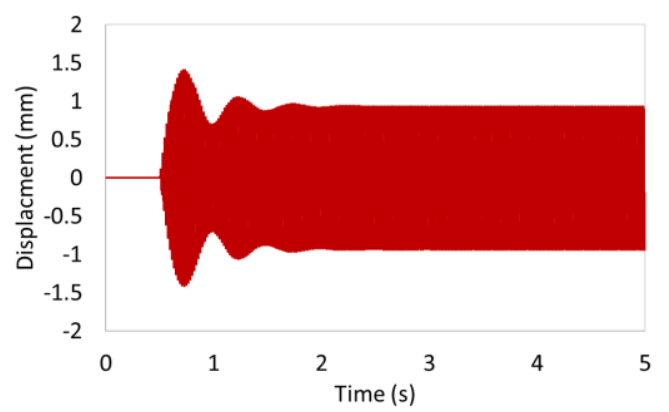

(a)

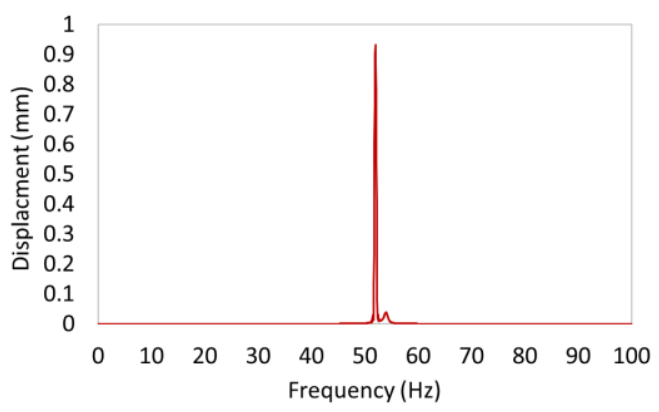

(b)

Figure B.4.3: Theoretical response displacement (a) time history and (b) frequency spectra for $52 \mathrm{~Hz}$ excitation.

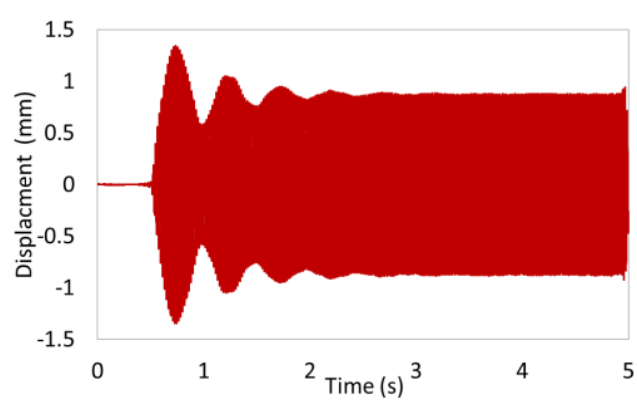

(a)

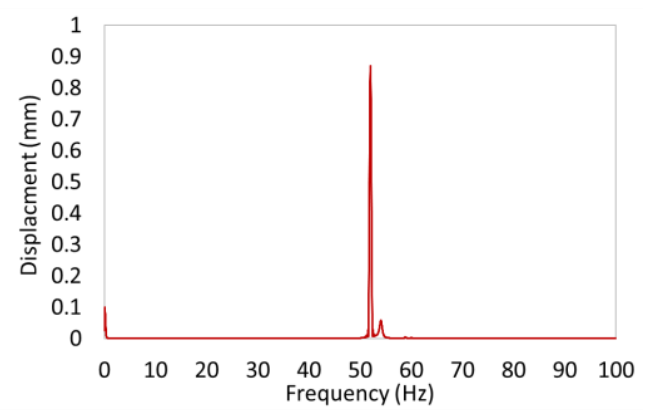

(b)

Figure B.4.4: Experimental response displacement (a) time history and (b) frequency spectra for $52 \mathrm{~Hz}$ excitation. 


\section{B.5 Displacement input and response for $54 \mathrm{~Hz}$ excitation}

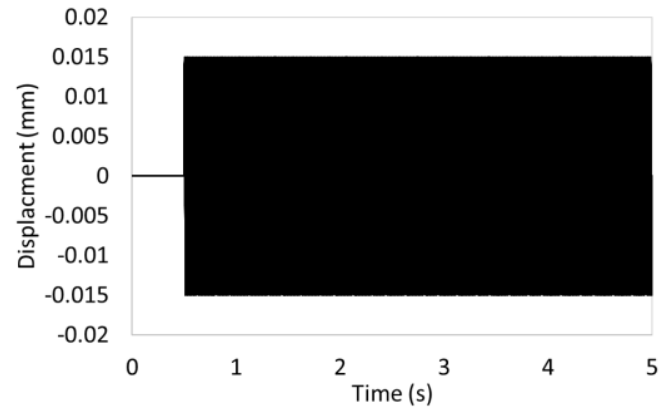

(a)

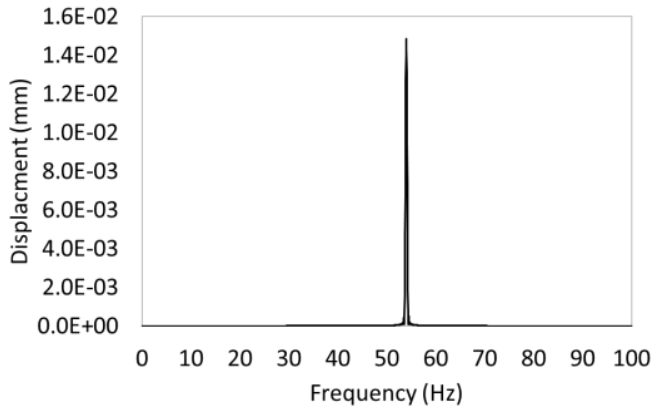

(b)

Figure B.5.1: Theoretical input displacement (a) time history and (b) frequency spectra for $54 \mathrm{~Hz}$ excitation.

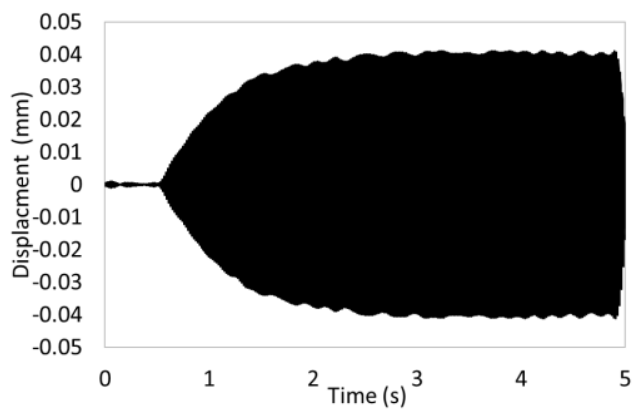

(a)

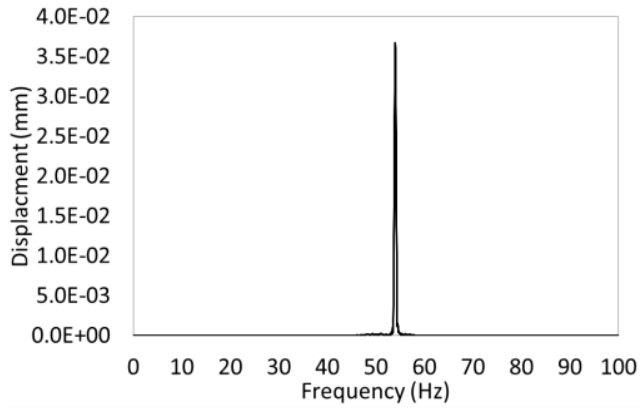

(b)

Figure B.5.2: Experimental input displacement (a) time history and (b) frequency spectra for 54

Hz excitation. 


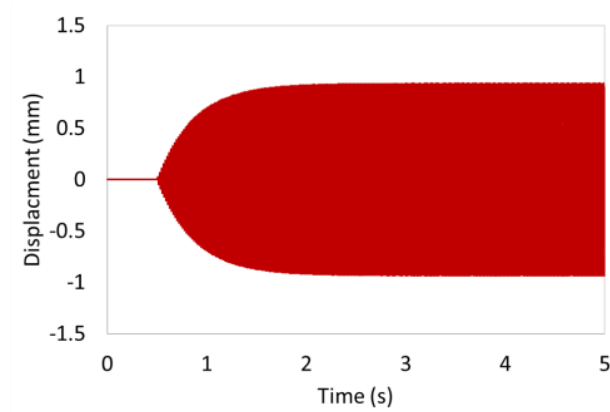

(a)

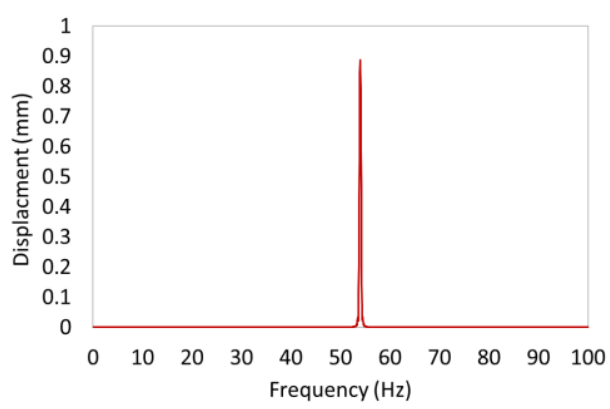

(b)

Figure B.5.3: Theoretical response displacement (a) time history and (b) frequency spectra for $54 \mathrm{~Hz}$ excitation.

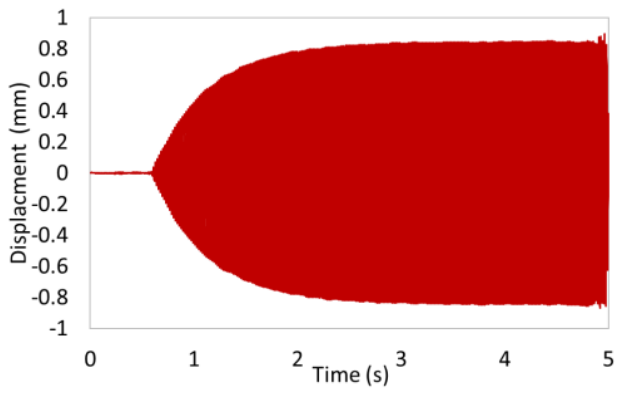

(a)

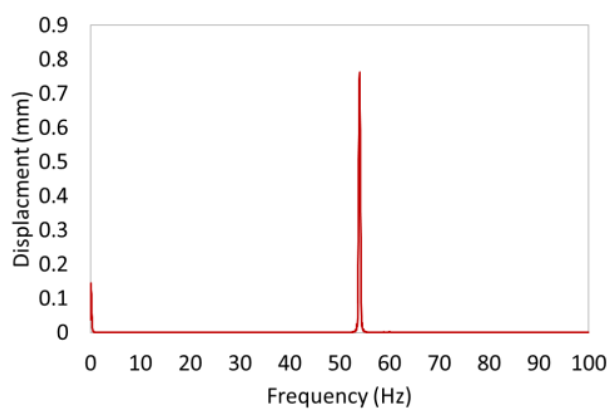

(b)

Figure B.5.4: Experimental response displacement (a) time history and (b) frequency spectra for $54 \mathrm{~Hz}$ excitation. 


\section{B.6 Displacement input and response for $56 \mathrm{~Hz}$ excitation}

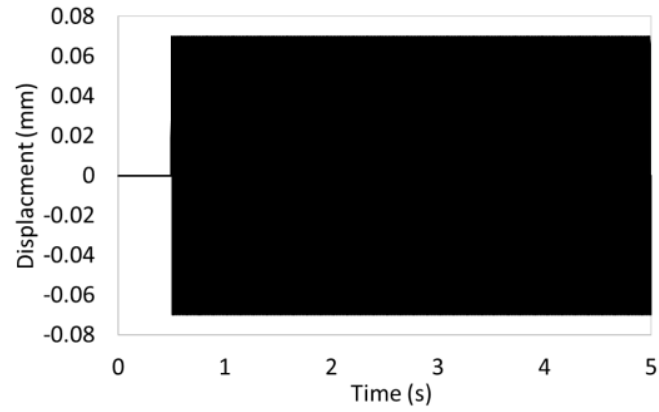

(a)

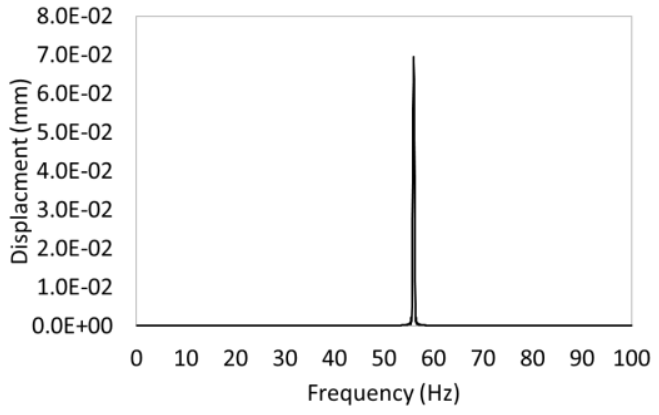

(b)

Figure B.6.1: Theoretical input displacement (a) time history and (b) frequency spectra for $56 \mathrm{~Hz}$ excitation.

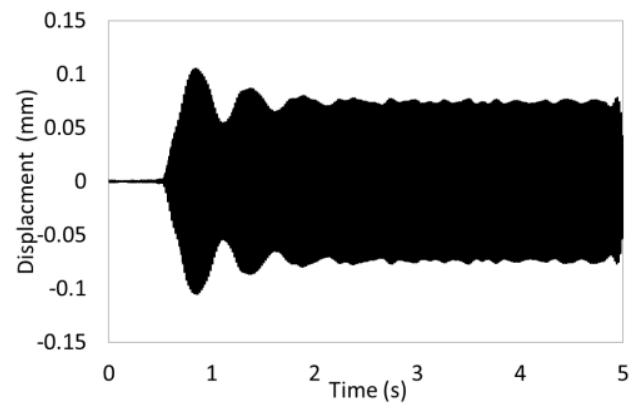

(a)

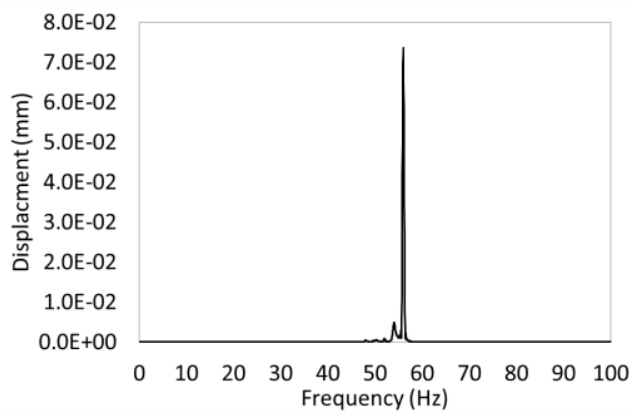

(b)

Figure B.6.2: Experimental input displacement (a) time history and (b) frequency spectra for 56

Hz excitation. 


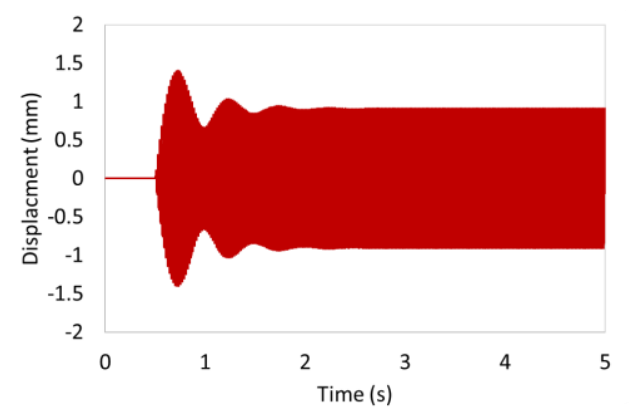

(a)

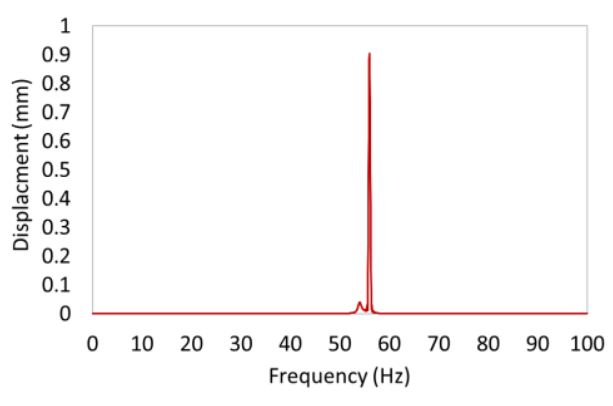

(b)

Figure B.6.3: Theoretical response displacement (a) time history and (b) frequency spectra for $56 \mathrm{~Hz}$ excitation.

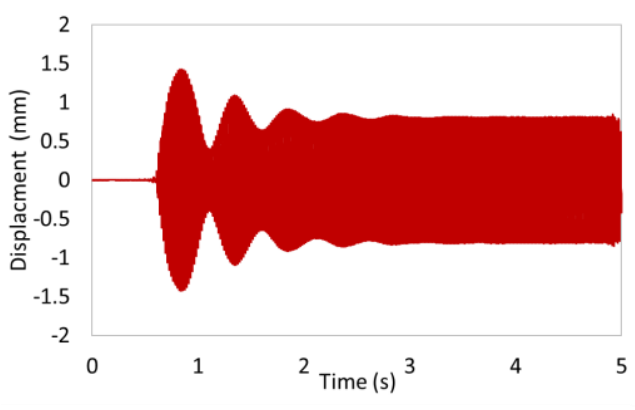

(a)

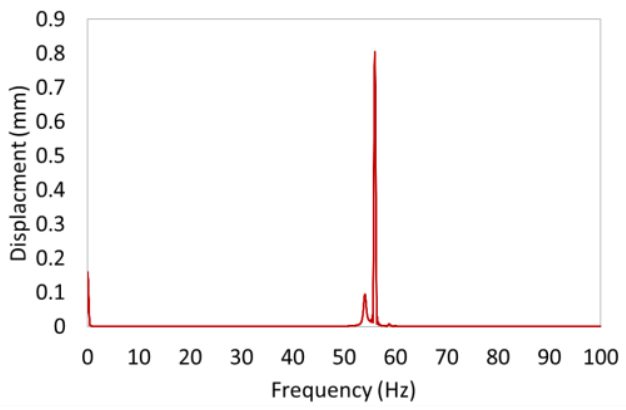

(b)

Figure B.6.4: Experimental response displacement (a) time history and (b) frequency spectra for $56 \mathrm{~Hz}$ excitation. 


\section{B.7 Displacement input and response for $58 \mathrm{~Hz}$ excitation}

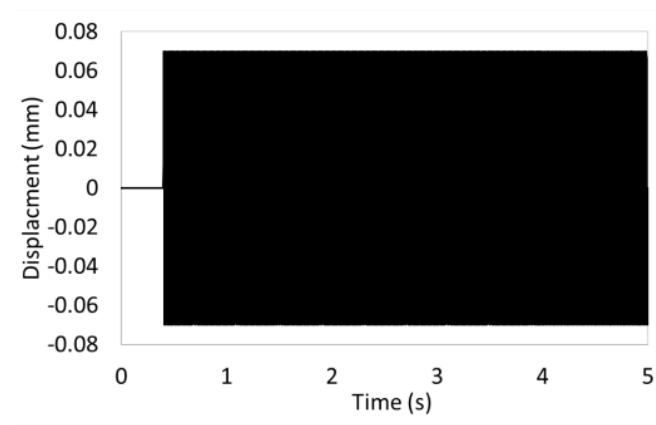

(a)

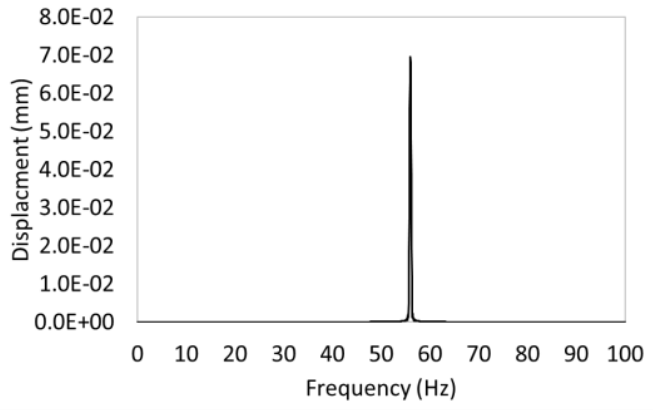

(b)

Figure B.7.1: Theoretical input displacement (a) time history and (b) frequency spectra for $58 \mathrm{~Hz}$ excitation.

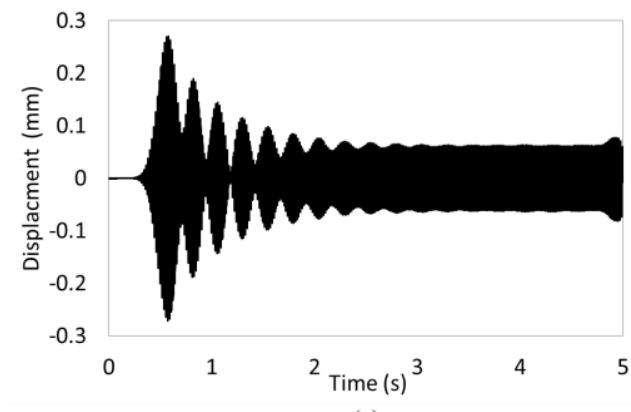

(a)

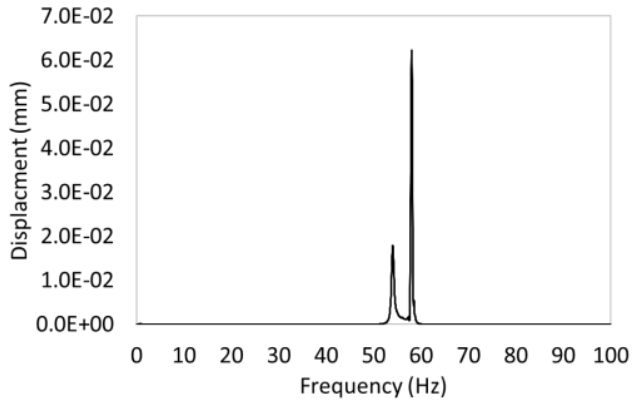

(b)

Figure B.7.2: Experimental input displacement (a) time history and (b) frequency spectra for 58

Hz excitation. 


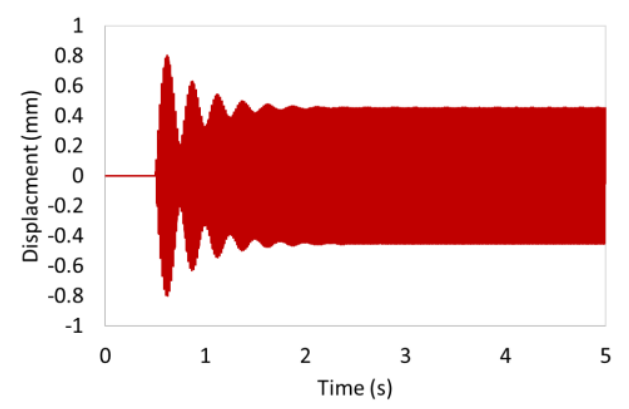

(a)

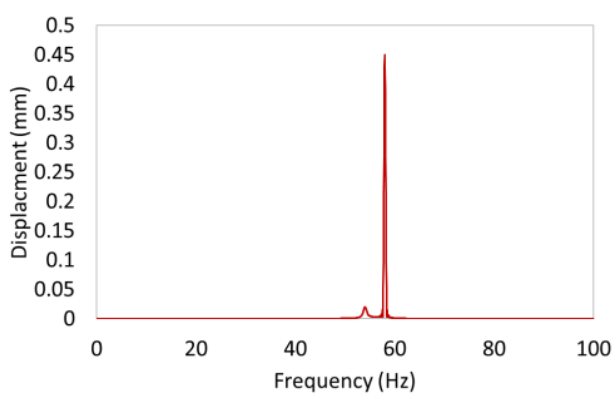

(b)

Figure B.7.3: Theoretical response displacement (a) time history and (b) frequency spectra for $58 \mathrm{~Hz}$ excitation.

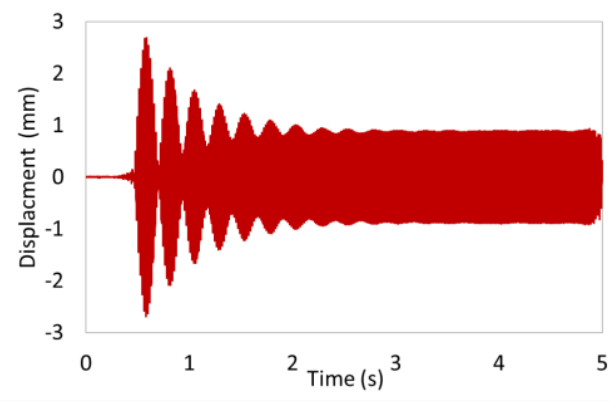

(a)

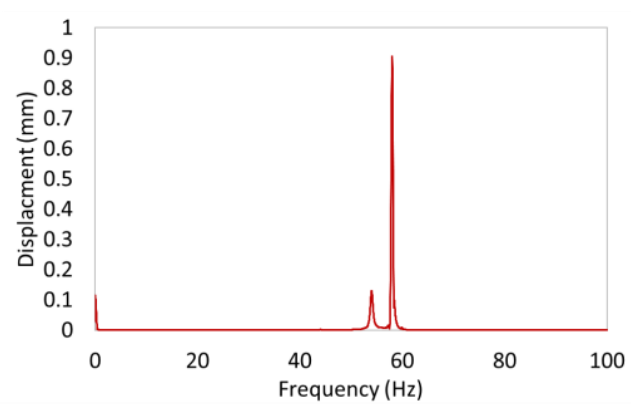

(b)

Figure B.7.4: Experimental response displacement (a) time history and (b) frequency spectra for $58 \mathrm{~Hz}$ excitation. 


\section{B.8 Displacement and EMF charts for SR14}

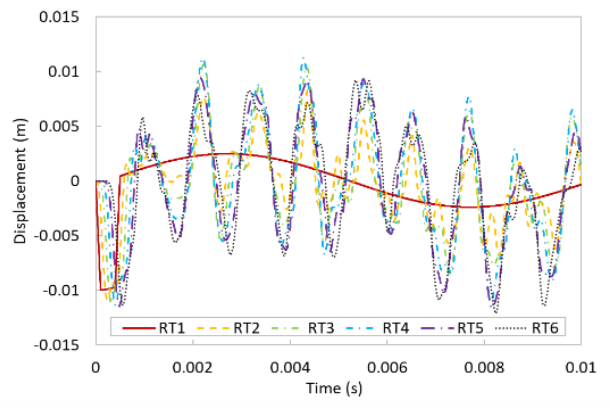

(a)

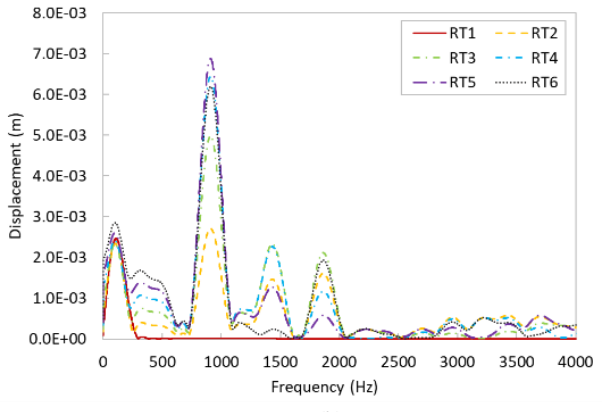

(b)

Figure B.8.1: Displacement response (a) time history and (b) frequency spectra for SR14, $100 \mathrm{~Hz}$ resonator sets $(6 \times 100 \mathrm{~Hz})$

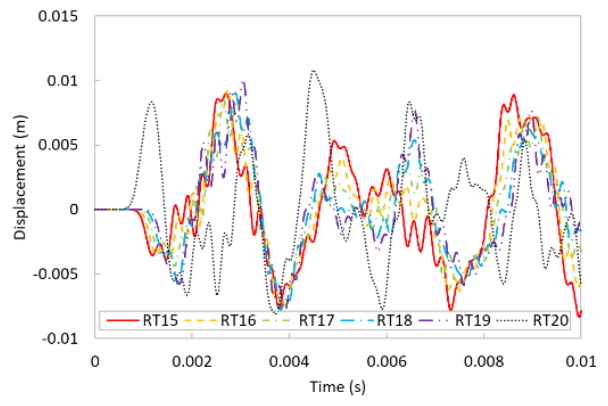

(a)

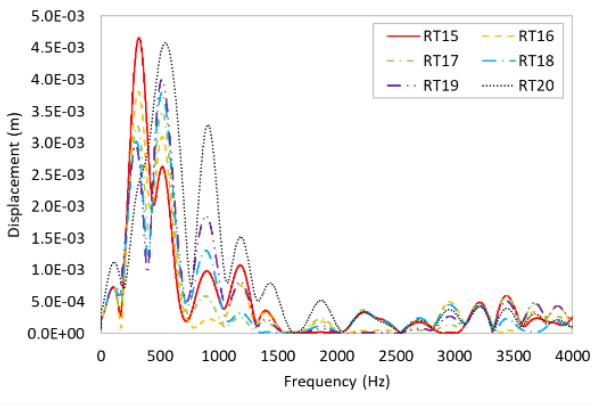

(b)

Figure B.8.2: Displacement response (a) time history and (b) frequency spectra for SR14, $300 \mathrm{~Hz}$ resonator sets $(6 \times 300 \mathrm{~Hz})$ 


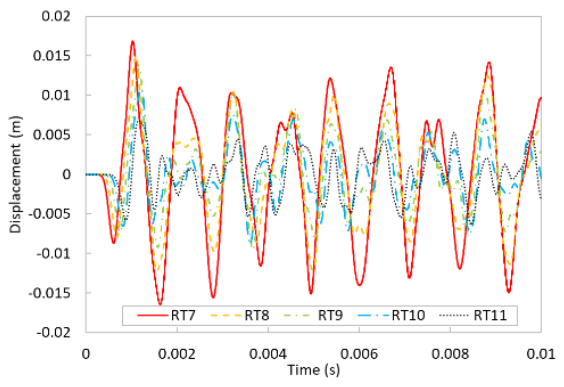

(a)

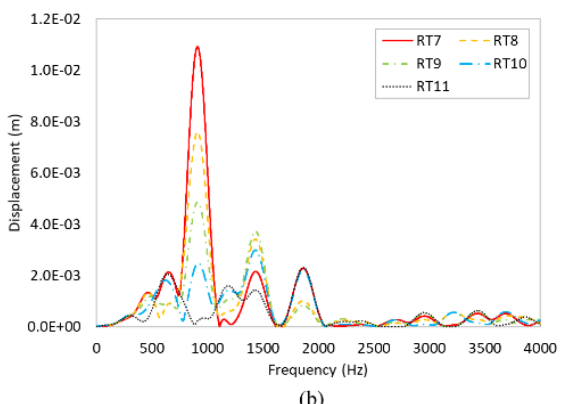

(b)

Figure B.8.3: Displacement response (a) time history and (b) frequency spectra for $\mathrm{SR} 14,700 \mathrm{~Hz}$ resonator sets $(5 x 700 \mathrm{~Hz})$

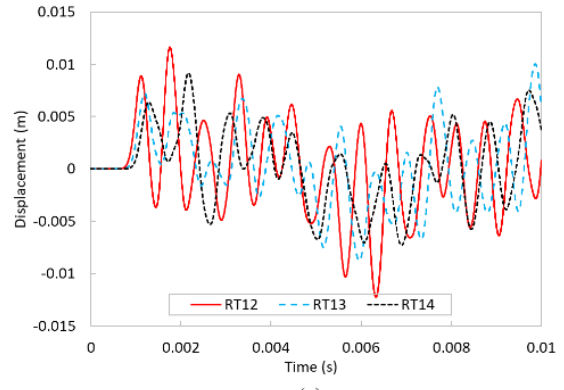

(a)

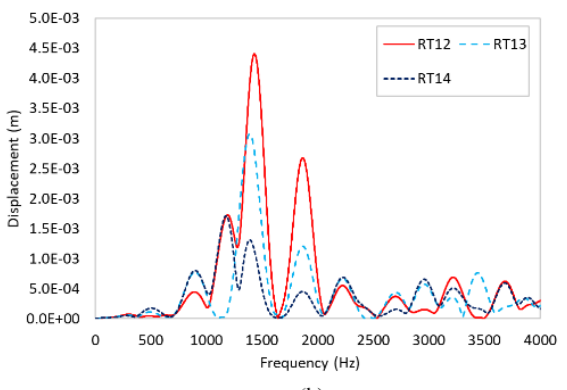

(b)

Figure B.8.4: Displacement response (a) time history and (b) frequency spectra for SR14, 1500 $\mathrm{Hz}$ resonator sets $(3 \times 1500 \mathrm{~Hz})$
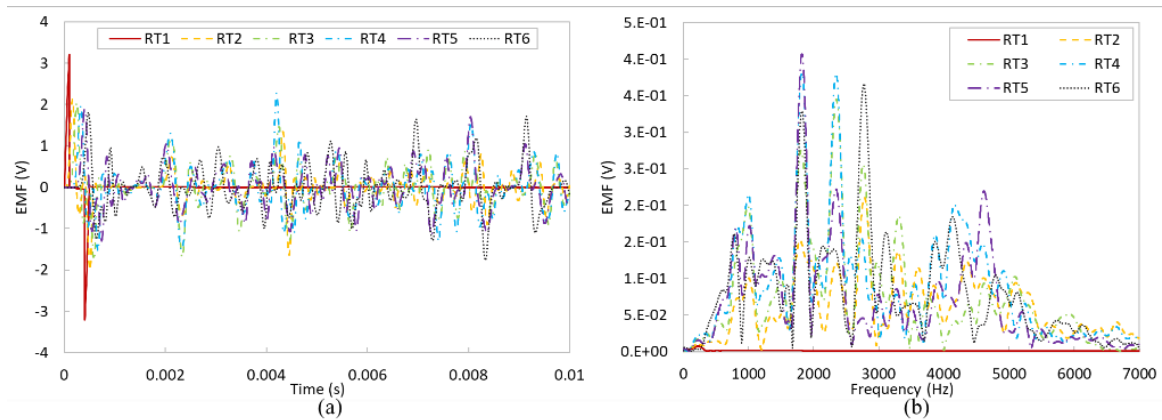

Figure B.8.5: Harvested EMF (a) time history and (b) frequency spectra for SR14, $100 \mathrm{~Hz}$ resonator sets $(6 \times 100 \mathrm{~Hz})$ 


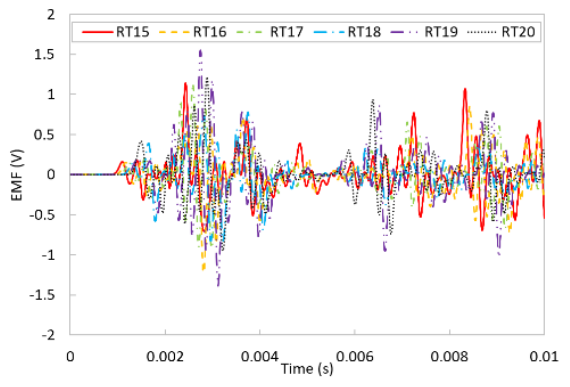

(a)

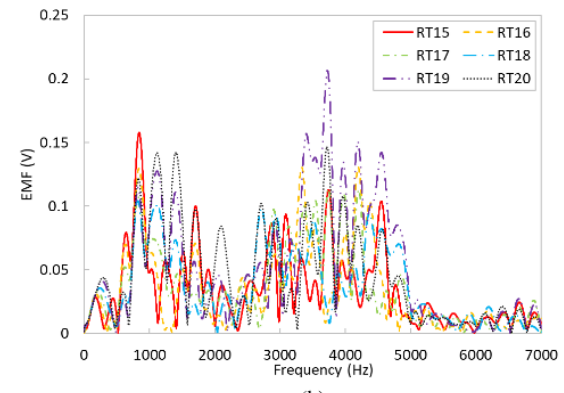

(b)

Figure B.8.6: Harvested EMF (a) time history and (b) frequency spectra for SR14, $300 \mathrm{~Hz}$ resonator sets $(6 x 300 \mathrm{~Hz})$

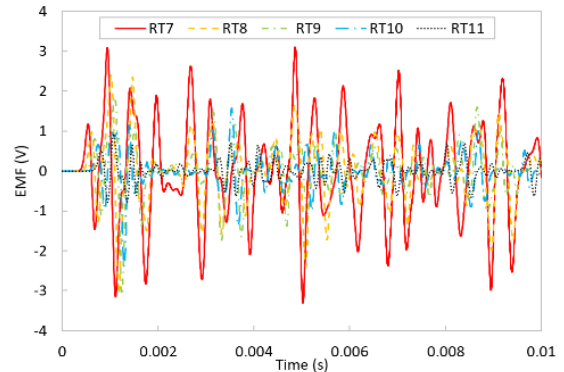

(a)

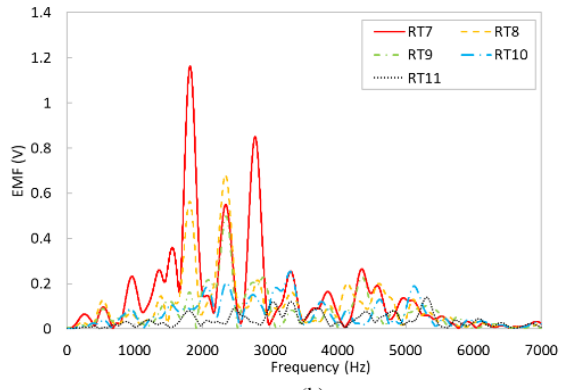

(b)

Figure B.8.7: Harvested EMF (a) time history and (b) frequency spectra for SR14, $700 \mathrm{~Hz}$ resonator sets $(5 x 700 \mathrm{~Hz})$

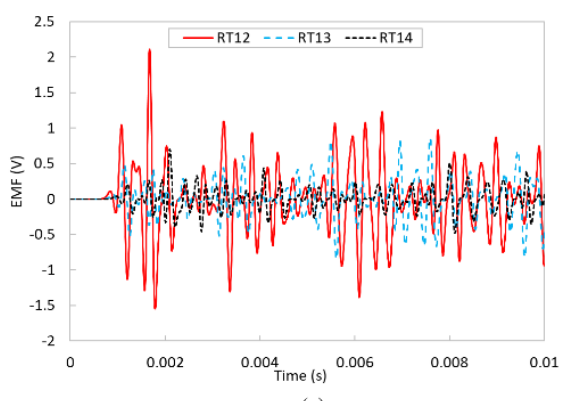

(a)

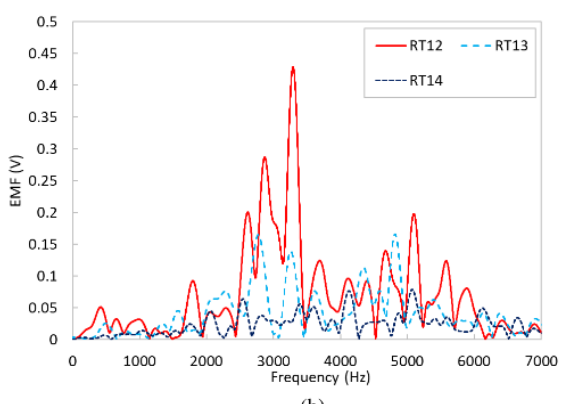

(b)

Figure B.8.8: Harvested EMF (a) time history and (b) frequency spectra for SR14, $1500 \mathrm{~Hz}$ resonator sets $(3 \times 1500 \mathrm{~Hz})$ 


\section{B.9 Displacement and EMF charts for SQ6}

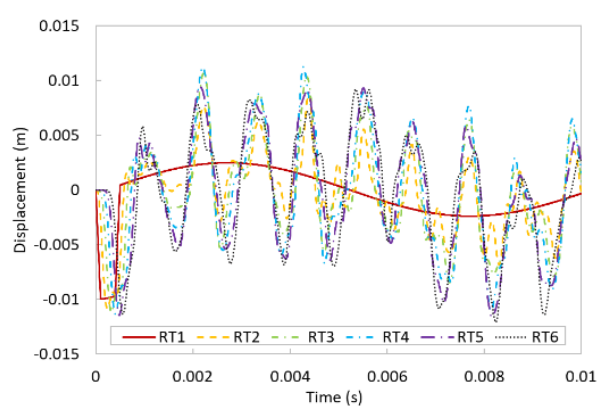

(a)

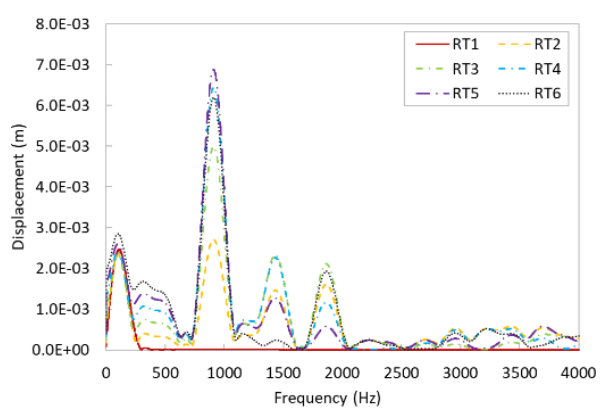

(b)

Figure B.9.1: Displacement response (a) time history and (b) frequency spectra for $S Q 6,100 \mathrm{~Hz}$ resonator sets $(6 x 100 \mathrm{~Hz})$

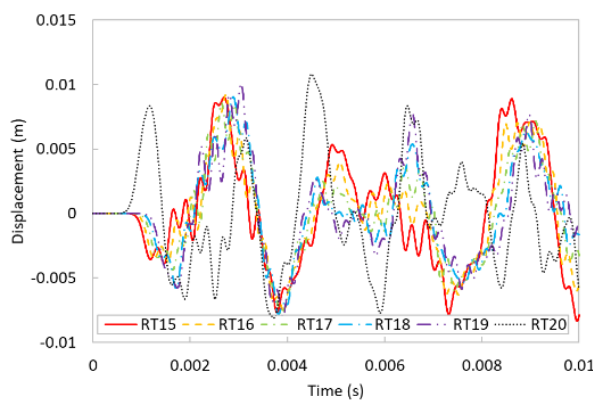

(a)

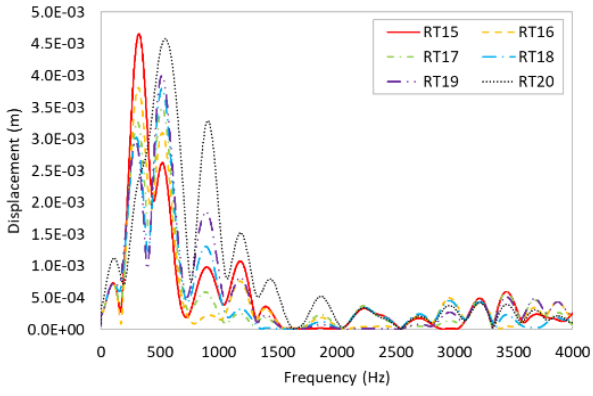

(b)

Figure B.9.2: Displacement response (a) time history and (b) frequency spectra for SQ6, $300 \mathrm{~Hz}$ resonator sets $(6 x 300 \mathrm{~Hz})$ 


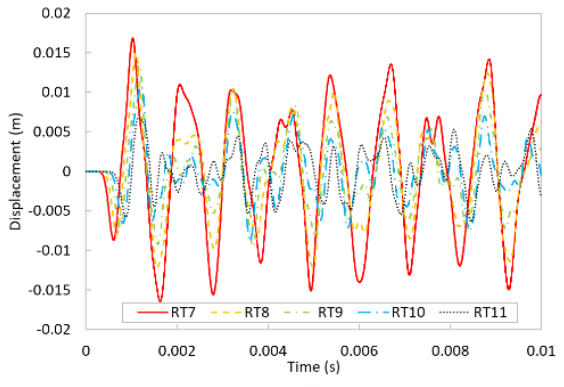

(a)

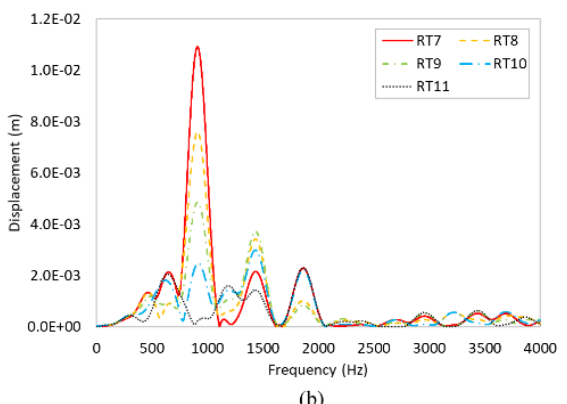

(b)

Figure B.9.3: Displacement response (a) time history and (b) frequency spectra for $S Q 6,700 \mathrm{~Hz}$ resonator sets $(5 \times 700 \mathrm{~Hz})$

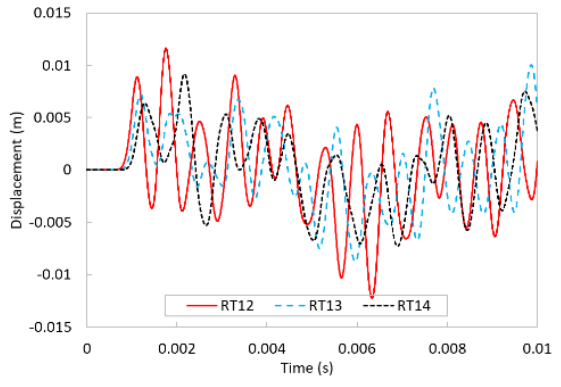

(a)

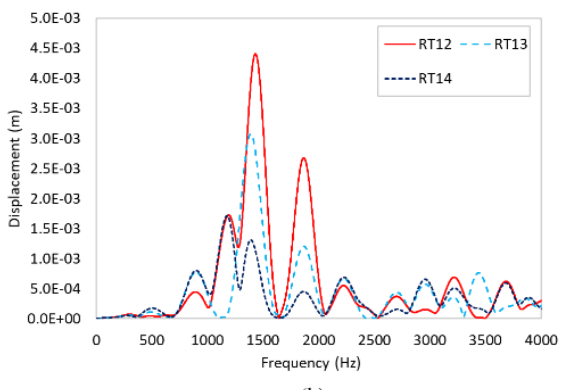

(b)

Figure B.9.4: Displacement response (a) time history and (b) frequency spectra for SQ6, 1500 $\mathrm{Hz}$ resonator sets $(3 \times 1500 \mathrm{~Hz})$
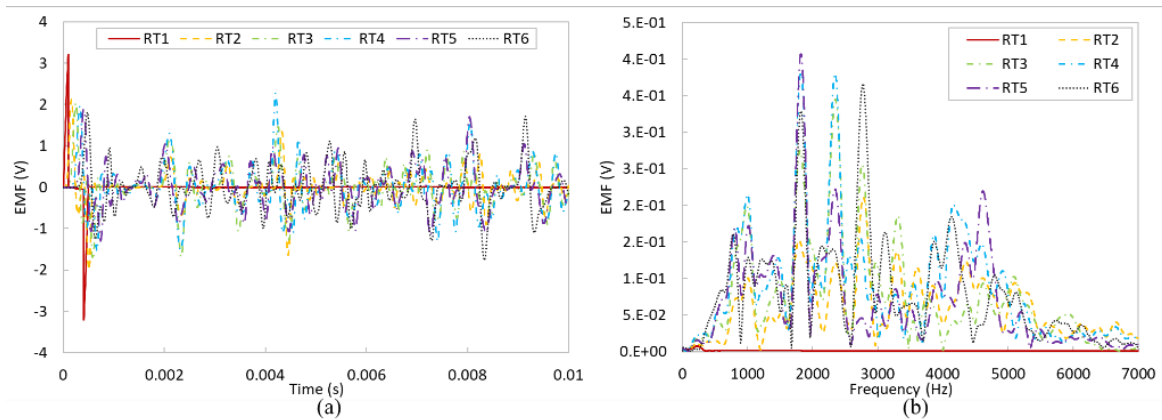

Figure B.9.5: Harvested EMF (a) time history and (b) frequency spectra for $S Q 6,100 \mathrm{~Hz}$ resonator sets $(6 \times 100 \mathrm{~Hz})$ 


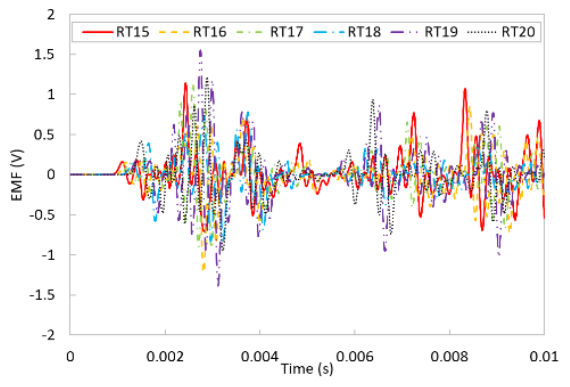

(a)

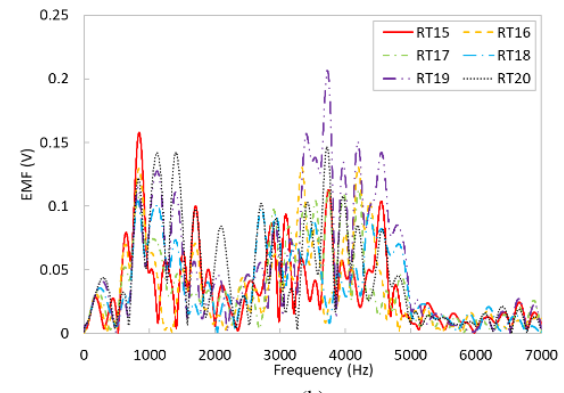

(b)

Figure B.9.6: Harvested EMF (a) time history and (b) frequency spectra for SQ6, $300 \mathrm{~Hz}$ resonator sets $(6 \times 300 \mathrm{~Hz})$

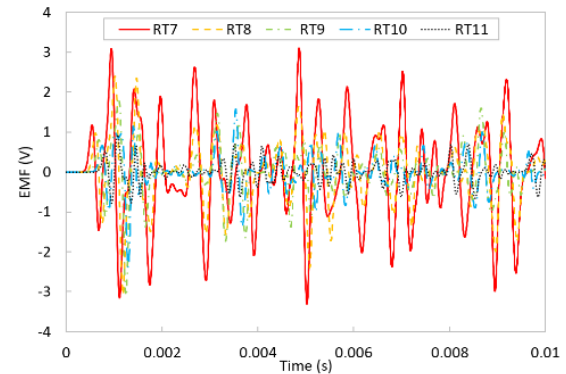

(a)

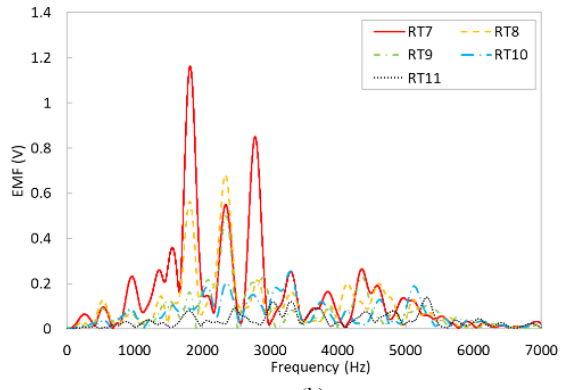

(b)

Figure B.9.7: Harvested EMF (a) time history and (b) frequency spectra for SQ6, $700 \mathrm{~Hz}$ resonator sets $(5 x 700 \mathrm{~Hz})$

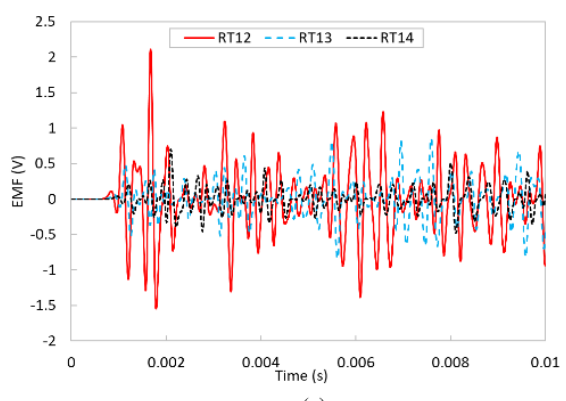

(a)

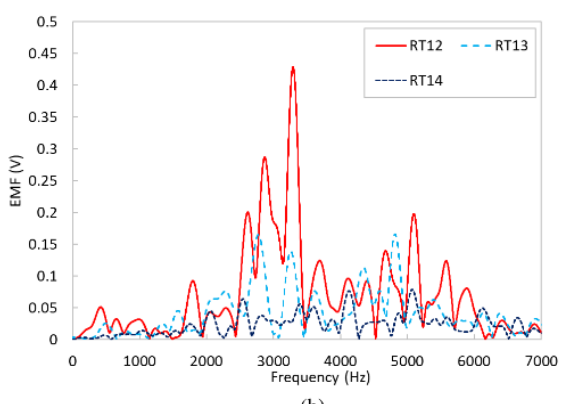

(b)

Figure B.9.8: Harvested EMF (a) time history and (b) frequency spectra for SQ6, $1500 \mathrm{~Hz}$ resonator sets $(3 \times 1500 \mathrm{~Hz})$ 


\section{B.10 Displacement and EMF charts for SM6}

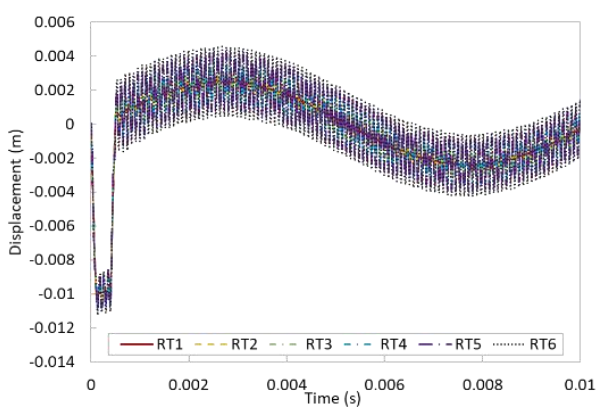

(a)

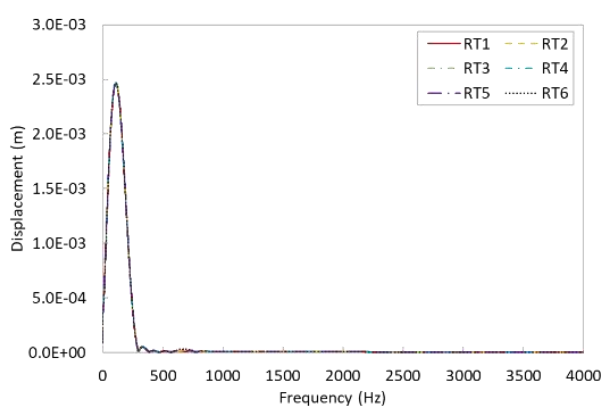

(b)

Figure B.10.1: Displacement response (a) time history and (b) frequency spectra for SM6, 100 $\mathrm{Hz}$ resonator sets $(6 \times 100 \mathrm{~Hz})$
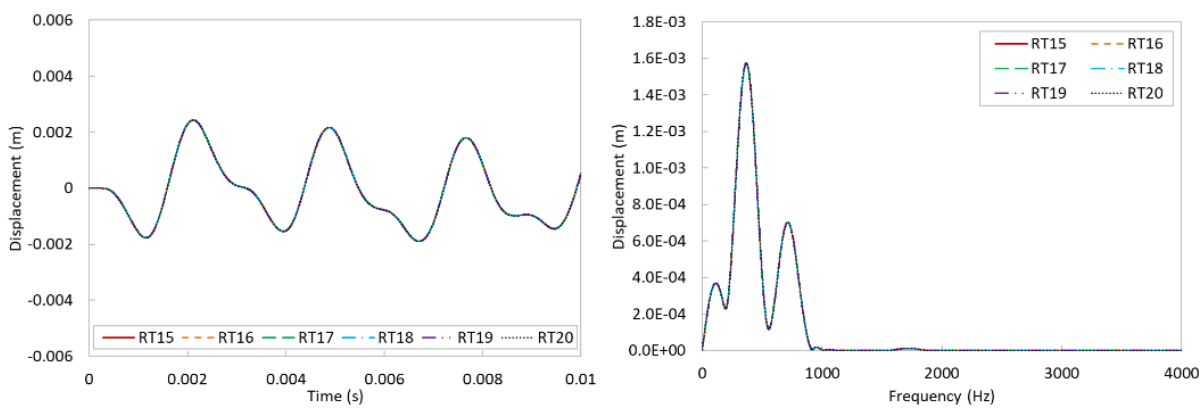

Figure B.10.2: Displacement response (a) time history and (b) frequency spectra for SM6, 300 $\mathrm{Hz}$ resonator sets $(6 x 300 \mathrm{~Hz})$ 

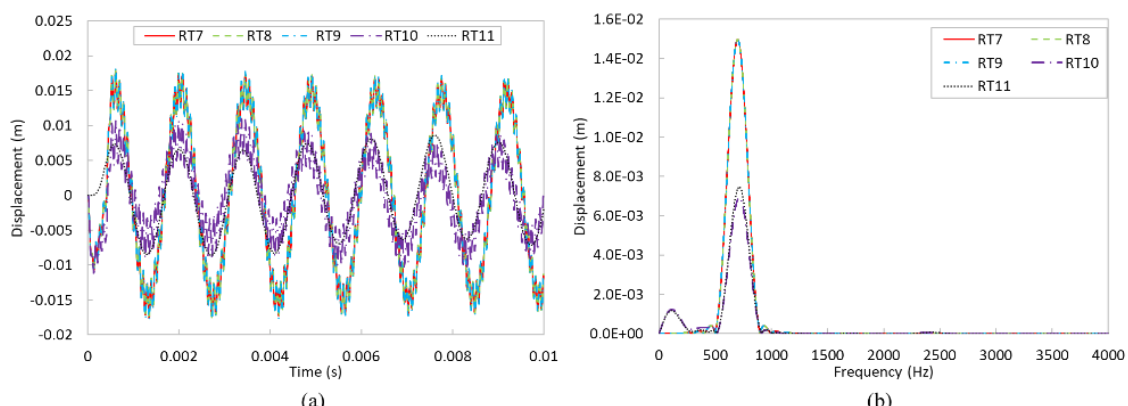

Figure B.10.3: Displacement response (a) time history and (b) frequency spectra for SM6, 700 $\mathrm{Hz}$ resonator sets $(5 x 700 \mathrm{~Hz})$

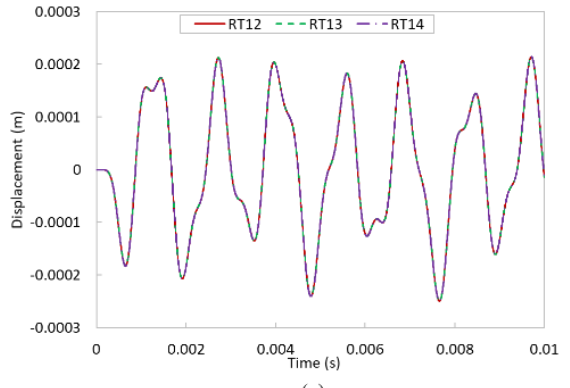

(a)

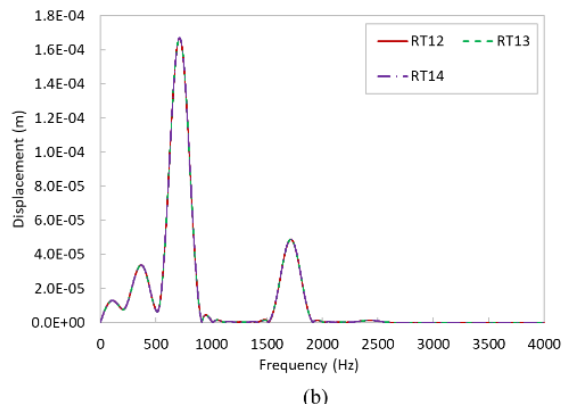

(b)

Figure B.10.4: Displacement response (a) time history and (b) frequency spectra for SM6, 1500 $\mathrm{Hz}$ resonator sets $(3 \times 1500 \mathrm{~Hz})$

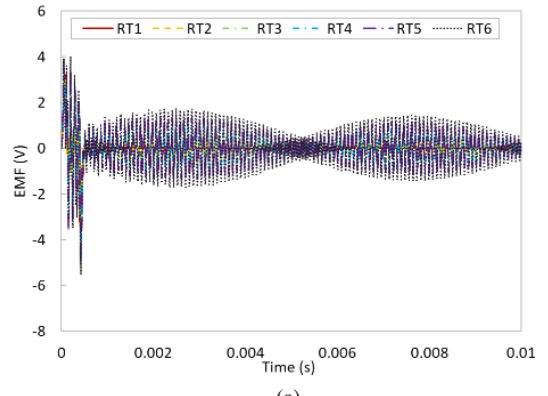

(a)

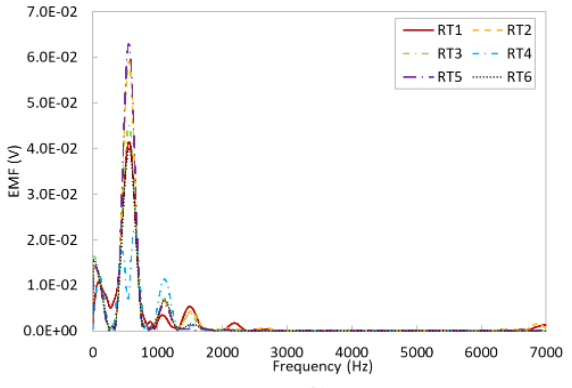

(b)

Figure B.10.5: Harvested EMF (a) time history and (b) frequency spectra for SM6, $100 \mathrm{~Hz}$ resonator sets $(6 \times 100 \mathrm{~Hz})$ 


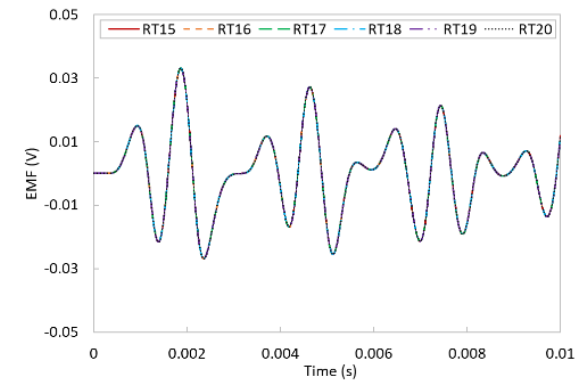

(a)

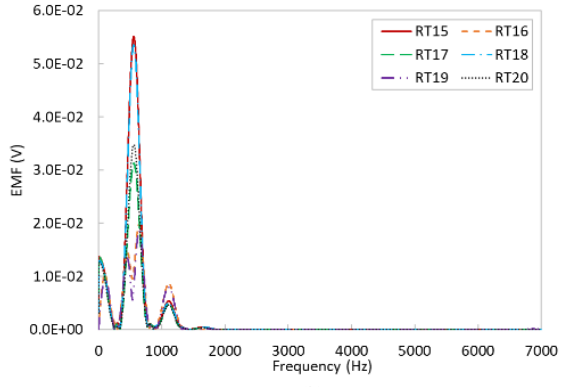

(b)

Figure B.10.6: Harvested EMF (a) time history and (b) frequency spectra for SM6, $300 \mathrm{~Hz}$ resonator sets $(6 \times 300 \mathrm{~Hz})$

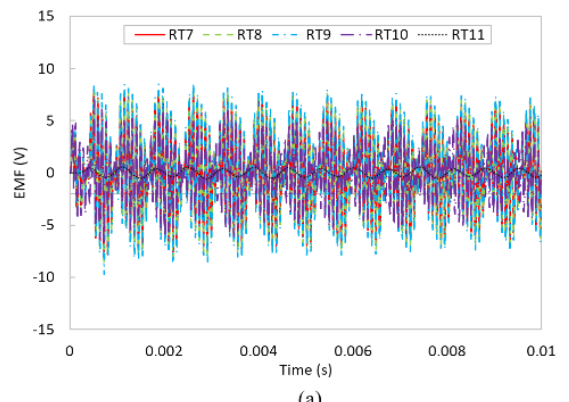

(a)

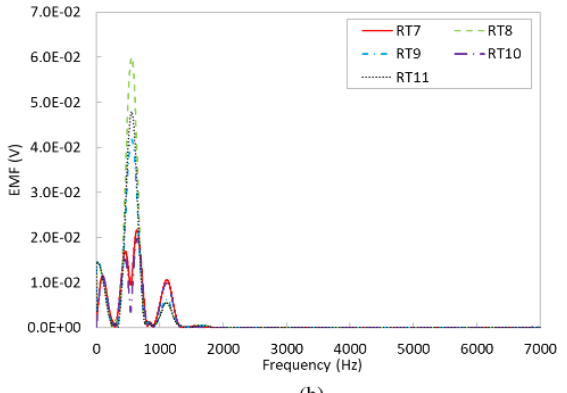

(b)

Figure B.10.7: Harvested EMF (a) time history and (b) frequency spectra for SM6, $700 \mathrm{~Hz}$ resonator sets $(5 x 700 \mathrm{~Hz})$

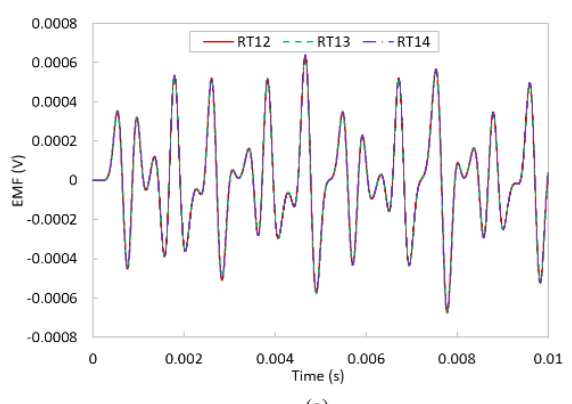

(a)

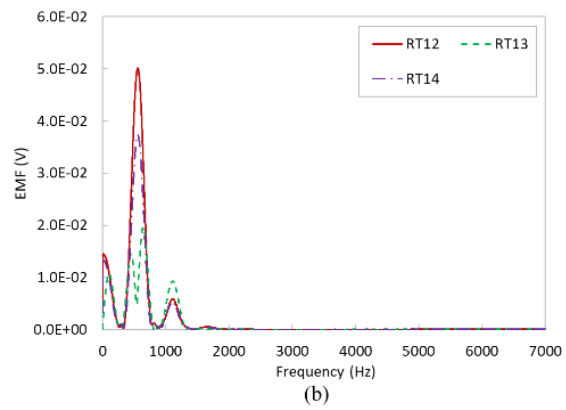

Figure B.10.8: Harvested EMF (a) time history and (b) frequency spectra for SM6, $1500 \mathrm{~Hz}$ resonator sets $(3 \times 1500 \mathrm{~Hz})$ 
VITA

Milkyas Afework

Candidate for the Degree of

Master of Science

\section{Thesis: EXPLORING MECHANICAL ASSONANCE FOR IMPACT ENERGY HARVESTING USING ACOUSTIC METAMATERIALS}

Major Field: Mechanical and Aerospace Engineering

Biographical:

Education:

Completed the requirements for the Master of Science in Mechanical and Aerospace Engineering at Oklahoma State University, Oklahoma in December, 2020.

Completed the requirements for the Bachelor of Science in Mechanical Engineering at Oklahoma State University, Oklahoma in 2018.

Experience:

Graduate Research Assistant / Lab Manager

Solid and Structural Dynamics Lab

School of Mechanical and Aerospace Engineering

Oklahoma State University, Stillwater, OK, 74078

Conference Publications and Presentations

1. M. Afework, P. P. Kulkarni and J. M. Manimala, "Assonance: A Design Framework for Targeted Spectral Transformations in Acoustic Metamaterials" 10/2019

INCE's Noise-Con Conference

2. M. Afework and J. M. Manimala, "Robust Heuristics-Enabled, Vision-Based Sensor for Enhanced Automobile Safety" $10 / 2019$

International Digital Image Correlation

Society's Conference and Workshop 